\author{
Universidade de São Paulo \\ Instituto de Física
}

\title{
Testes de aspectos do Modelo Supersimétrico por meio da interação de neutrinos de altíssimas energias com a Terra
}

\author{
Jairo Cavalcante de Souza \\ Tese de doutorado apresentada ao \\ Instituto de Física da Universidade \\ de São Paulo para a obtenção do \\ título de Doutor em Ciências.
}

Orientadora: Profa. Dra. Ivone Freire da Mota e Albuquerque

Comissão Examinadora

Prof. Dr. Arthur Kos Antunes Maciel (CBPF)

Prof. Dr. Ernesto Kemp (IFGW-Unicamp)

Profa. Dra. Ivone Freire da Mota e Albuquerque (IFUSP)

Profa. Dra. Marina Nielsen (IFUSP)

Prof. Dr. Philippe Gouffon (IFUSP)

São Paulo

2012 


\section{FICHA CATALOGRÁFICA}

\section{Preparada pelo Serviço de Biblioteca e Informação}

\section{do Instituto de Física da Universidade de São Paulo}

Souza, Jairo Cavalcante de

Testes de aspectos do Modelo Supersimétrico por meio da interação de neutrinos de altíssimas energias com a Terra - São Paulo, 2012.

Tese (Doutorado) - Universidade de São Paulo.

Instituto de Física, Depto. Física Experimental.

Orientador: $\operatorname{Prof}^{a} \operatorname{Dr}^{a}$ Ivone Freire da Mota e Albuquerque

Área de Concentração: Física

Unitermos: 1. Física Moderna; 2. Física Nuclear;

3. Física de Partículas; 4. Neutrinos; 5. Supersimetria.

USP/IF/SBI-027/2012 
Dedico esta tese à Pri, companheira em todos os

momentos. 
"A saudade é a maior prova de que o passado valeu a pena" Pablo Neruda 


\section{Sumário}

Agradecimentos

Resumo iii

$\begin{array}{ll}\text { Abstract } & \text { v }\end{array}$

1 Introdução 1

2 Detecção direta de NLSPs $\quad 9$

2.1 Fluxo de neutrinos . . . . . . . . . . . . . . . . . 11

2.2 Seção de choque neutrino-núcleon - Léptons . . . . . . . . . . 13

2.3 Seção de choque neutrino-núcleon - NLSP . . . . . . . . . . . 15

2.4 Produção de (s)léptons . . . . . . . . . . . . . . . . . . . 18

2.4.1 Perfil de densidade da Terra . . . . . . . . . . . . 22

2.5 Perda de energia de (s)léptons na Terra . . . . . . . . . . 25

2.5.1 Bremsstrahlung . . . . . . . . . . . . . . 28

2.5.2 Produção de Pares . . . . . . . . . . . . . . . . . . . . 29

2.5.3 Interação fotonuclear . . . . . . . . . . . . . . . 31

2.5.4 Alcance máximo de (s)léptons na Terra . . . . . . . 35

2.6 Simulação de eventos no detector . . . . . . . . . . . . . . 37

2.7 Resultados . . . . . . . . . . . . . . . . . . . . . 39

3 Detecção Indireta de NLSPs: IceCube 43

3.1 Decaimento da NLSP . . . . . . . . . . . . . . . . . . . . . . . 44

3.1.1 Probabilidade de decaimento do stau . . . . . . . . 45

3.1.2 Cinemática do decaimento do stau . . . . . . . . . 47

3.2 Regeneração do tau . . . . . . . . . . . . . . . . . . . . . . 48

3.3 Simulação de eventos . . . . . . . . . . . . . . . . . . . . . 51 
3.3.1 Múons provenientes do decaimento do tau . . . . . . 54

3.4 Resultados . . . . . . . . . . . . . . . . . . . 54

3.4.1 Taus originados em decaimentos de NLSPs . . . . . . 55

3.4.2 Taus de decaimentos de NLSPs - IceCube ampliado . . 65

3.4.3 Produção direta de taus . . . . . . . . . . . . . 76

4 Detecção Indireta de NLSPs - JEM-EUSO 85

4.1 Simulação de eventos . . . . . . . . . . . . . . . . . . . . 87

4.2 Fluorescência atmosférica . . . . . . . . . . . . . . . . . . . 89

4.3 Resultados . . . . . . . . . . . . . . . . . . . 90

4.3.1 Fluxo e energia na atmosfera e energia . . . . . . . . 90

4.3.2 Distância e tempo entre os decaimentos . . . . . . . . 97

5 Discussão 101

6 Conclusões 109

A Telescópio IceCube 111

$\begin{array}{ll}\text { B Parametrizações } & 115\end{array}$

B.1 Seção de choque - produção de léptons . . . . . . . . . . . . . 116

B.2 Seção de choque - produção de NLSPs . . . . . . . . . . . . . 118

B.3 Perda de energia: múon, tau e stau . . . . . . . . . . . . . 120

B.4 Parametrização de y . . . . . . . . . . . . . . . . . . . 126

$\begin{array}{ll}\text { C Distribuição aleatória } & 129\end{array}$

$\begin{array}{lc}\text { D Cinemática } & 133\end{array}$

D.1 Convenções adotadas . . . . . . . . . . . . . . . . . . 133

D.2 Decaimento em dois corpos . . . . . . . . . . . . . . 135

D.3 Boost do CM para Lab . . . . . . . . . . . . . . . . . . 136

D.3.1 Determinação de $\gamma_{C M}$ e de $v_{C M} \ldots \ldots$. . . . . . . . . 139

D.3.2 Determinação das velocidades no CM . . . . . . . . . . 140

D.3.3 Determinação dos momentos no CM . . . . . . . . 141

D.3.4 Determinação da energia do CM . . . . . . . . . . . . . 142

E Simulação de trajetórias 
SUMÁRIO

$\begin{array}{ll}\text { Referências Bibliográficas } & 147\end{array}$

$\begin{array}{ll}\text { Lista de Figuras } & 150\end{array}$

Lista de Tabelas 159 
SUMÁRIO 


\section{Agradecimentos}

Agradeço à Professora Dra. Ivone F. M. Albuquerque por todos os anos de orientação e pelo apoio em minhas tomadas de decisão importantes durante este doutorado.

Gostaria de agradecer também ao amigo Diogo Bernardes Tridapalli pelas discussões que influenciaram diretamente no sucesso deste trabalho.

Por fim, agradeço à Fapesp pelo apoio financeiro. 


\section{Resumo}

O principal objetivo deste trabalho é propor testes para alguns aspectos do modelo padrão supersimétrico mínimo (MSSM), proposto como extensão do modelo padrão de física de partículas. Mais especificamente, testamos cenários de quebra de supersimetria mediada por interações de calibre (gauge). Mostramos como determinar limites para a escala de quebra de supersimetria e formas de detectar indiretamente partículas supersimétricas. Propomos testes a serem realizados por telescópios de neutrinos como também por telescópios de fluorescência atmosférica. Nestes cenários as partículas supersimétricas se originam a partir da interação de neutrinos cosmológicos de altíssimas energias com núcleons no interior da Terra. Determinamos, a partir de simulações Monte Carlo, os sinais a serem detectados nestes telescópios.

A detecção indireta destas partículas é baseada em seu decaimento, que pode ocorrer no interior da Terra ou na atmosfera, produzindo um tau mais um gravitino. Propomos aqui como testar estes cenários, tanto com a detecção de taus em telescópios de neutrinos bem como em telescópios de fluorescência atmosférica. Como os decaimentos estão ligados a uma janela restrita para a escala de quebra de supersimetria, entre $\sim 10^{5} \mathrm{GeV}$ e $\sim 10^{7}$ $\mathrm{GeV}$, propomos não somente formas de detectar partículas supersimétricas, mas também, como testar a escala de quebra de supersimetria nos cenários considerados. Mostramos aqui que é possível observar sinais provocados por partículas supersimétricas nas duas situações experimentais avaliadas. 


\section{Abstract}

The main goal of this research is to propose probes of aspects of the minimal supersymmetric standard model (MSSM), assuming gauge mediated supersymmetry breaking scenarios. More specifically, how to determine limits for the supersymmetry breaking energy scale and ways for indirect detection of supersymmetric particles. We propose tests to be carried out both by neutrino telescopes as well as by atmospheric fluorescence. Under these scenarios, supersymmetric particles originate from ultrahigh energy cosmological neutrinos interacting with nucleons inside the Earth. Through Monte Carlo simulations, we determine the signatures will be left in these telescopes.

The indirect detection of these particles is based in their decay, which can occur inside the Earth or at the atmosphere, producing a tau plus a gravitino. Here we propose how to probe these scenarios, either by detecting taus, generated in the decay, in neutrino telescopes or in atmospheric fluorescence telescopes. As these decays are connected to a restrict window for the supersymmetry breaking energy scale, which would be between $\sim 10^{5} \mathrm{GeV}$ and $\sim 10^{7} \mathrm{GeV}$, we are proposing not only ways of detecting supersymmetric particles as well as how to probe the supersymmetry breaking energy scale in the scenarios considered. We show here that it is possible to observe these signals in two setups evaluated. 


\section{Capítulo 1}

\section{Introdução}

O principal objetivo deste trabalho é propor testes para alguns aspectos de cenários de quebra de supersimetria mediada por interações de calibre (gauge) relativos ao modelo padrão supersimétrico mínimo (MSSM), mais especificamente, como determinar limites para a escala de quebra de supersimetria e formas de detectar indiretamente partículas supersimétricas. Propomos testes a serem realizados por telescópios de neutrinos como também por telescópios de fluorescência atmosférica. Nestes cenários as partículas supersimétricas se originam a partir da interação de neutrinos cosmológicos de altíssimas energias com núcleons no interior da Terra. Determinamos, a partir de simulações Monte Carlo, os sinais a serem detectados nestes telescópios.

O modelo padrão da física de partículas (MP) prevê com excelente precisão a descrição de fenômenos que ocorrem até a escala TeV. Suas previsões teóricas foram comprovadas em vários laboratórios, dentre os quais o Fermilab e o CERN. Entretanto, limitações do MP já começam a aparecer na descrição de fenômenos que ocorrem em escalas de energias em torno de centenas de GeVs. Uma destas limitações surge devido às correções radiativas advindas do acoplamento, direto ou indireto, de partículas ao campo de Higgs, que contribuem da seguinte forma para sua massa:

$$
\Delta m_{H}^{2} \propto\left|\lambda^{2}\right| \Lambda_{U V}^{2}+\ldots
$$


em que $\Lambda_{U V}$ representa a escala de energia limite (cutoff) para a qual o MP é válido. Se o MP for válido até a escala de Planck tem-se $\Lambda_{U V} \sim$ $m_{\text {Planck }}$. O parâmetro $\lambda$ é a constante de acoplamento de uma partícula ao campo de Higgs. A maior contribuição a $\Delta m_{H}$ vem do acoplamento com o quark top, onde $\lambda \sim 1$. Isto implica que para $\Lambda_{U V} \sim m_{\text {Planck }}$, a massa do Higgs deveria atingir a escala de Planck $\left(10^{19} \mathrm{GeV}\right)$. No entanto, as medidas experimentais limitam a massa do Higgs, indicando que ela está dentro do intervalo 114,4 $\leq m_{H} \leq 197 \mathrm{GeV}$ com 99\% de nível de confiança [1]. Este comportamento observado no MP é conhecido como problema da hierarquia $[2,3]$, e por isso se diz que o MP é instável sob correções radiativas na escala eletrofraca [4]. Este é um dos motivos pelos quais se espera uma extensão do MP já nessa escala de energia.

A teoria supersimétrica na escala eletrofraca é uma das fortes candidatas a esta extensão. A supersimetria resolve o problema da hierarquia do MP, isto é, as divergências devido às correções radiativas, ao introduzir uma nova simetria [4] que relaciona estados bosônicos com estados fermiônicos. Isto implica na existência de um parceiro supersimétrico para cada partícula conhecida do MP. O parceiro supersimétrico será um bóson quando a partícula do MP for um férmion e vice-versa. Com esta nova simetria as divergências quadráticas nas correções radiativas se cancelam, dado que para cada contribuição há uma equivalente de sinal oposto proveniente do parceiro supersimétrico. Esta simetria não pode ser exata, caso contrário as partículas supersimétricas já teriam sido encontradas visto que suas massas seriam as mesmas de suas parceiras do MP. Desta forma, a escala de massa das partículas supersimétricas deve ser maior do que a escala de massa do MP. Isto indica que esta nova simetria deve ser quebrada. Porém, diferentemente do MP, neste caso as correções radiativas provenientes de acoplamento ao campo de Higgs são menores, logarítmicas e não quadráticas, como veremos adiante.

O Modelo Padrão Supersimétrico Mínimo (MSSM, de Minimal Supersymmetric Standard Model) propõe uma composição de supermultipletos chirais nos quais campos de spin zero são campos escalares e campos de spin $1 / 2$ são férmions de Weyl de duas componentes de mão esquerda. As tabelas 1.1 e 1.2 (obtidas de [4]) ilustram o espectro de massas para este modelo. 
Tabela 1.1: Supermultipletos chirais no modelo padrão supersimétrico mínimo. Os campos de spin zero são escalares complexos e os campos de spin $1 / 2$ são férmions de Weyl de mão esquerda de duas componentes. Tabela obtida de [4].

\begin{tabular}{|c|c|c|c|c|}
\hline \multicolumn{2}{|l|}{ Nomes } & spin 0 & spin $1 / 2$ & $S U(3)_{C} \times S U(2)_{L} \times U(1)_{Y}$ \\
\hline (s)quarks & $Q$ & $\left(\begin{array}{ll}\widetilde{u}_{L} & \widetilde{d}_{L}\end{array}\right)$ & $\left(\begin{array}{ll}u_{L} & d_{L}\end{array}\right)$ & $\left(\mathbf{3}, \mathbf{2}, \frac{1}{6}\right)$ \\
\hline ( ×3 famílias $)$ & $\bar{u}$ & $\widetilde{u}_{R}^{*}$ & $u_{R}^{\dagger}$ & $\left(\overline{\mathbf{3}}, \mathbf{1},-\frac{2}{3}\right)$ \\
\hline & $\bar{d}$ & $\widetilde{d}_{R}^{*}$ & $d_{R}^{\dagger}$ & $\left(\overline{\mathbf{3}}, \mathbf{1}, \frac{1}{3}\right)$ \\
\hline (s)léptons & $L$ & $\left(\widetilde{\nu} \widetilde{e}_{L}\right)$ & $\left(\begin{array}{ll}\nu & e_{L}\end{array}\right)$ & $\left(1,2,-\frac{1}{2}\right)$ \\
\hline (×3 famílias $)$ & $\bar{e}$ & $\widetilde{e}_{R}^{*}$ & $e_{R}^{\dagger}$ & $(\mathbf{1}, \mathbf{1}, 1)$ \\
\hline Higgs(inos) & $H_{u}$ & $\left(\begin{array}{ll}H_{u}^{+} & H_{u}^{0}\end{array}\right)$ & $\left(\begin{array}{ll}\widetilde{H}_{u}^{+} & \widetilde{H}_{u}^{0}\end{array}\right)$ & $\left(\mathbf{1}, \mathbf{2},+\frac{1}{2}\right)$ \\
\hline & $H_{d}$ & $\left(\begin{array}{ll}H_{d}^{0} & H_{d}^{-}\end{array}\right)$ & $\left(\begin{array}{ll}\widetilde{H}_{d}^{0} & \widetilde{H}_{d}^{-}\end{array}\right)$ & $\left(\mathbf{1}, \mathbf{2},-\frac{1}{2}\right)$ \\
\hline
\end{tabular}

Supermultipletos de gauge no MSSM:

Tabela 1.2: Supermultipletos de gauge no modelo padrão supersimétrico mínimo. Tabela obtida de [4].

\begin{tabular}{|c|c|c|c|}
\hline Nomes & spin $1 / 2$ & spin 1 & $S U(3)_{C} \times S U(2)_{L} \times U(1)_{Y}$ \\
\hline \hline gluíno, glúon & $\widetilde{g}$ & $g$ & $(\mathbf{8}, \mathbf{1}, 0)$ \\
\hline winos, bósons W & $W^{ \pm} \widetilde{W}^{0}$ & $W^{ \pm} W^{0}$ & $(\mathbf{1}, \mathbf{3}, 0)$ \\
\hline bino, bóson B & $\widetilde{B}^{0}$ & $B^{0}$ & $(\mathbf{1}, \mathbf{1}, 0)$ \\
\hline
\end{tabular}

O mecanismo de quebra de supersimetria utilizado no MSSM para a determinação do espectro de massas envolve a introdução de uma lagrangiana efetiva:

$$
\mathcal{L}=\mathcal{L}_{S U S Y}+\mathcal{L}_{\text {soft }}
$$

onde $\mathcal{L}_{S U S Y}$ contém todas as interações de Yukawa e de gauge e preserva invariância por supersimetria, enquanto $\mathcal{L}_{\text {soft }}$ viola supersimetria. As partículas com massas maiores do que as parceiras do MP pertencem a este último termo e são da ordem de $m_{\text {soft }} \sim \mathrm{TeV}$. Desta forma as correções para o acoplamento ao campo de Higgs são dadas por: 


$$
\Delta m_{H}^{2} \propto m_{\text {soft }}^{2}|\lambda| \ln \left(\Lambda_{U V} / m_{\text {soft }}\right)+\ldots
$$

onde as correções adicionais não supersimétricas a $\Delta m_{H}^{2}$ tendem a zero no limite $m_{\text {soft }} \rightarrow 0$, preservando a invariância por supersimetria. Assim as correções radiativas advindas da contribuição dos acoplamentos das partículas supersimétricas ao campo de Higgs são muito menores do que no MP, deixando de ser quadráticas, e no mecanismo de quebra de simetria eletrofraca presente no MSSM passam a ser logarítmicas.

Dentro do espectro de partículas definido pela quebra de supersimetria estão a partícula supersimétrica mais leve (LSP - Lightest Supersymmetric Particle) e a seguinte mais leve (NLSP - Next to Lightest Supersymmetric Particle). A composição da LSP depende da escala de energia da quebra de supersimetria, denotada por $\sqrt{F}$. Geralmente em cenários nos quais $\sqrt{F} \gtrsim 10^{10} \mathrm{GeV}$, a LSP é o neutralino, já quando $\sqrt{F} \lesssim 10^{10} \mathrm{GeV}$, a LSP é o gravitino. Tipicamente a LSP é uma partícula neutra e estável, característica assegurada por uma simetria discreta presente no modelo chamada de paridade-R (ou equivalentemente, "paridade de matéria") [4]. É uma simetria que não parte do pressuposto de que o número bariônico $(B)$ e o número leptônico $(L)$ sejam conservados, porém é um número quântico multiplicativamente conservado para cada partícula da teoria, definido da seguinte forma:

$$
P_{R}=(-1)^{3(B-L)+2 s}
$$

em que $s$ é o spin da partícula. As partículas supersimétricas têm $P_{R}=-1$, enquanto as do MP, $P_{R}=+1$. Para que esta paridade seja exatamente conservada, não é possível que as partículas supersimétricas se misturem com as de $P_{R}=+1$. Entre as consequências fenomenológicas importantes está o fato de a LSP não decair.

Neste trabalho nos concentraremos em cenários de supersimetria onde o gravitino é a LSP e a NLSP é um slépton, isto é, o parceiro supersimétrico de um lépton. Um grande número de modelos supersimétricos adotam este cenário, como por exemplo, as teorias com quebra de supersimetria mediada por interação de gauge [5]. Nestes casos, a NLSP é tipicamente o stau de 
mão direita $\left(\tilde{\tau}_{R}\right)$.

Recentemente foi mostrado [6] que é possível detectar as NLSPs em telescópios de neutrinos por meio da interação de neutrinos cosmológicos com núcleons no interior da Terra. Esta interação produz pares destas partículas supersimétricas que atingem o detector, como ilustrado na figura 1.1. Seus sinais experimentais foram analisados e comparados ao fundo composto de eventos de dimuons [7]. O telescópio IceCube ${ }^{1}$ [8] que fica no Polo Sul tem uma área efetiva que o torna capaz de detectar algumas unidades destas partículas por ano e de separá-las do fundo.

A detecção das NLSPs seria não só importante pela própria descoberta destas partículas, como também por definir uma janela de energia onde se dá a quebra de supersimetria. Para os resultados obtidos em $[6,7]$ temos uma restrição para esta janela de energia tal que $10^{7} \mathrm{GeV} \lesssim \sqrt{F} \lesssim 10^{10} \mathrm{GeV}$. Além disso, teríamos um forte indício de que a matéria escura seria composta pelo gravitino.

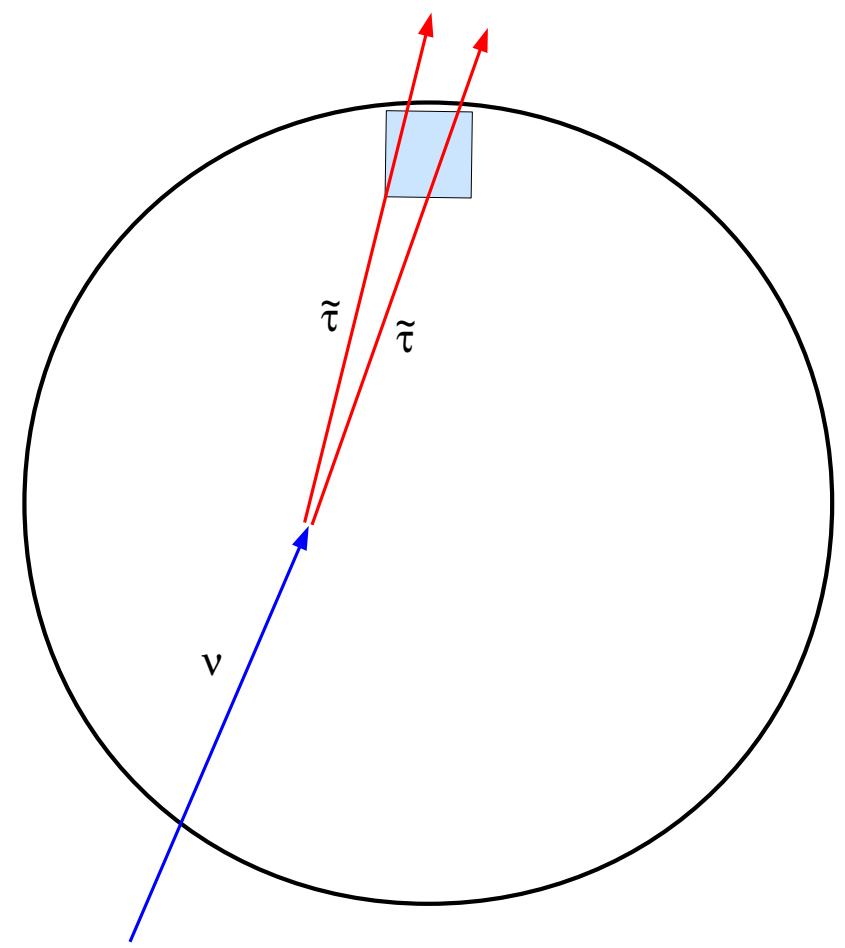

Figura 1.1: Esquema da interação de neutrinos cosmológicos com a Terra produzindo duas NLSPs que atingem um telescópio de neutrinos.

\footnotetext{
${ }^{1}$ No apêndice A é apresentada uma breve descrição deste detector.
} 
Nesta tese complementamos a pesquisa em que se demonstrou $[6,7]$ a possibilidade de detecção direta das NLSPs em telescópios de neutrinos. Os limites assumidos para $\sqrt{F}$ naquela pesquisa implicam nas NLSPs serem estáveis, ou seja, elas não decaem enquanto viajam dentro da Terra. Aqui propomos uma análise complementar: assumimos que as NLSPs decaem (no interior da Terra ou na atmosfera após atravessarem o planeta), o que define um intervalo complementar para $\sqrt{F}$, com: $\sim 10^{5} \mathrm{GeV} \lesssim \sqrt{F} \lesssim 10^{7}$ $\mathrm{GeV}$. Desta forma ampliamos a região de quebra de supersimetria que pode ser determinada a partir da detecção direta ou indireta das NLSPs. Estas partículas devem ser produzidas na interação de neutrinos de altíssimas energias (energias maiores que $10^{14} \mathrm{eV}$ ) com a Terra. $\sqrt{F}$ será testada independentemente da detecção ou não da NLSP: se for detectada a $\sqrt{F}$ estará bem definida; caso contrário, esta região da $\sqrt{F}$ estará descartada. Aqui também a detecção das NLSPs será um indicativo do gravitino como matéria escura.

Analisamos duas possibilidades para o decaimento destas partículas:

i) decaimento no interior do planeta com a produção de um chuveiro passível de ser detectado pelo telescópio de neutrinos IceCube [8], ilustrado na figura 1.2;

ii) decaimento na atmosfera, após atravessarem o planeta, como ilustrado na figura 1.3. Neste caso temos a produção de um chuveiro atmosférico dentro do campo de visão do telescópio JEM-EUSO [9, 10], um detector a ser instalado na estação espacial internacional.

O decaimento das NLSPs no interior da Terra produz um tau e um gravitino. O tau decai rapidamente e um produto deste decaimento é o neutrino do tau, que por meio de interação de corrente carregada, pode interagir com um núcleon no interior da Terra e produzir um novo tau, o que caracteriza a regeneração do tau. Neste processo de regeneração, o novo tau é produzido com energia menor que o primeiro. Porém, o tau decai novamente em um neutrino do tau, e assim o processo continua. Determinamos aqui o fluxo de taus no detector (IceCube) bem como sua distribuição de energia. 


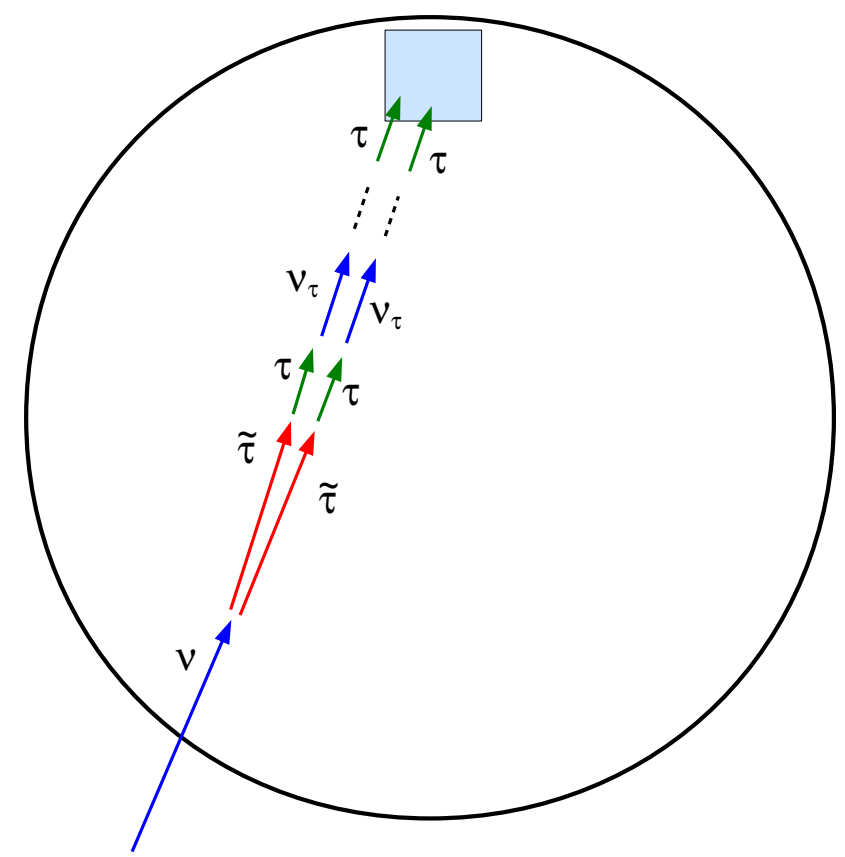

Figura 1.2: Esquema da interação de neutrinos cosmológicos com a Terra, produzindo duas NLSPs que decaem dentro do planeta.

Analisamos também os casos nos quais a produção/decaimento do tau ocorre após sua passagem pela Terra, ou seja, temos um neutrino cosmológico que incide sobre a Terra, interage em seu interior produzindo uma NLSP, que por sua vez se propaga no interior do Planeta, podendo atravessá-lo. Desta forma, ao contrário dos chuveiros atmosféricos gerados por colisões de partículas no topo da atmosfera, o chuveiro atmosférico produzido pelas NLSPs terá sua origem próxima à Terra e se desenvolverá "de baixo para cima" (figura 1.3). Neste caso, o interesse é que este chuveiro possa ser detectado pelo telescópio JEM-EUSO, por meio da fluorescência atmosférica produzida pelas partículas carregadas. Na possibilidade de o stau não decair dentro do campo de visão deste detector, foi analisada sua produção de fluorescência atmosférica, já que a NLSP é uma partícula carregada.

Esta tese está dividida da seguinte maneira: no capítulo 2 reproduzimos resultados de outros trabalhos presentes na literatura que determinam o fluxo de NLSPs e a correspondente distribuição de energia no IceCube. A partir do capítulo 3 passamos à parte original, onde são detalhados os passos para a obtenção do fluxo e distribuição de energia de taus no IceCube assim como 
a análise da possível detecção das NLSPs pelo JEM-EUSO. Na sequência é apresentada uma discussão sobre os resultados obtidos e depois as conclusões deste trabalho. Há também apêndices no final da tese, que detalham os procedimentos adotados nas simulações, assim como algumas deduções de geometria e de cinemática relativística.

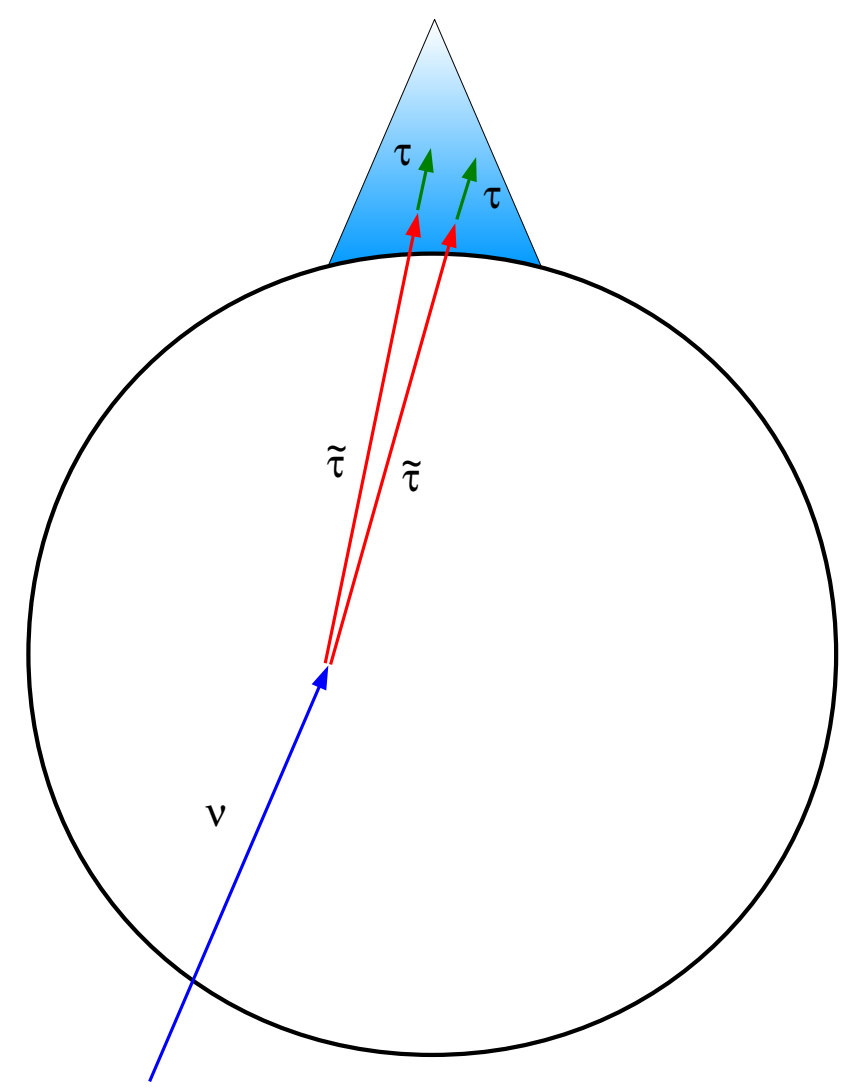

Figura 1.3: Esquema da interação de neutrinos cosmológicos com a Terra e produzindo duas NLSPs que decaem na atmosfera após atravessarem o planeta. 


\section{Capítulo 2}

\section{Detecção direta de NLSPs}

Aqui reproduzimos parte dos resultados [6,7] que demonstraram que é possível detectar pares de NLSPs em telescópios de neutrinos como o IceCube [8] e separá-los do fundo, composto principalmente por pares de múons (dimúons). Estas partículas supersimétricas seriam produzidas na interação de neutrinos cosmológicos de altas energias com núcleons no interior da Terra. Como mencionamos anteriormente, este resultado é importante não apenas pela possível descoberta de partículas supersimétricas como também por definir uma janela de energia para a quebra de supersimetria. Também constituiria a detecção indireta do gravitino, candidato a matéria escura.

O principal objetivo aqui é determinar a taxa de $\operatorname{NLSPs}^{1}$ que chegam em um telescópio de neutrinos com um volume de $1 \mathrm{~km}^{3}$ (como o IceCube), assim como os espectros de energia destas partículas ao chegarem ao detector. A reprodução destes resultados nos permitiu desenvolver as ferramentas (software) necessárias para a pesquisa original deste trabalho.

A taxa de NLSPs que atinge o detector, dada aqui em eventos por unidade de área e por unidade de tempo, é obtida por meio da convolução do fluxo de neutrinos que chega à Terra com as probabilidades do neutrino sobreviver e a de interagir, produzindo NLSPs, que dependem das interações neutrino-núcleon e de aspectos geométricos. Além disso, esta taxa também depende da perda de energia das NLSPs ao se propagarem no interior do planeta. De forma simplificada, a sequência de eventos a ser considerada para determinarmos a taxa de NLSPs é a seguinte: um fluxo de neutrinos, com

\footnotetext{
${ }^{1}$ Lembramos que nos cenários de supersimetria em que nos baseamos, a NLSP é constituída de sléptons, tipicamente o $\tilde{\tau}_{R}$.
} 
um determinado espectro de energia, chega à Terra. A probabilidade destes neutrinos interagirem com um núcleon no interior do planeta é determinada . Caso haja interação, determinamos a probabilidade de produção de pares de NLSPs. A perda de energia das NLSPs ao longo de sua trajetória pela Terra é levada em consideração para verificarmos se atingem o detector e com que energia.

Um dos aspectos que tornam a detecção direta de NLSPs possível está ligado ao fato de os staus serem capazes de percorrer distâncias muito grandes antes de decair. Nos modelos de quebra de supersimetria mediada por interação de gauge, esta distância é dada por [6, 7]:

$$
c \tau=\left(\frac{\sqrt{F}}{10^{7} G e V}\right)^{4}\left(\frac{100 G e V}{m_{\tilde{\tau}_{R}}}\right)^{5} 10 \mathrm{~km}
$$

Para $10^{7} \mathrm{GeV} \lesssim \sqrt{F} \lesssim 10^{10} \mathrm{GeV}$ as NLSPs decaem após atravessarem a Terra. A baixa perda de energia destas partículas garante que elas percorram grandes distâncias (de $\sim 50 \mathrm{~km}$ a $\sim 5 \times 10^{3} \mathrm{~km}$ ) antes de perder toda sua energia. Estas duas características aliadas compensam o fato de a seção de choque de produção das partículas supersimétricas ser muito menor do que a seção de choque para a produção de léptons, pois agregam um grande ganho em volume a favor das NLSPs. Outro aspecto importante é o fato de as NLSPs serem produzidas sempre aos pares e com uma energia muito alta, o que proporciona um ângulo de separação muito pequeno. Isto implica, dado que o alcance destas partículas é muito grande, que elas chegam ao detector como dois feixes paralelos, com uma separação de algumas centenas de metros, maior do que a observada com o principal fundo (dimúons), que é de algumas dezenas de metros.

Nas próximas seções deste capítulo são apresentados em detalhes os procedimentos e cálculos realizados para se chegar à taxa de eventos de NLSPs em telescópios de neutrinos, onde tomamos o IceCube como exemplo. Descrevemos o fluxo de neutrinos adotado neste trabalho; a determinação das probabilidades relativas à interação neutrino-núcleon; o cálculo da perda de energia das NLSPs e múons; e, por fim, as simulações Monte Carlo desenvolvidas para se chegar aos resultados apresentados. 


\subsection{Fluxo de neutrinos}

Para determinar a taxa de eventos de partículas supersimétricas no telescópio de neutrinos, é necessário conhecer o fluxo de neutrinos que chega à Terra. Neste trabalho consideramos como estimativas para o fluxo de neutrinos, o limite superior determinado por Waxman e Bahcall (WB) [11, 12].

No caso do limite de Waxman e Bahcall (WB) é pressuposto que os raios cósmicos de altíssimas energias são compostos por prótons. Eles se baseiam em observações de raios cósmicos de energias acima de $10^{17} \mathrm{eV}$ que indicam que o fluxo de raios cósmicos com energias acima de $\sim 3 \times$ $10^{18} \mathrm{eV}$ tem predomínio de prótons de origem extragaláctica, enquanto que para energias mais baixas é dominado por íons pesados, predominantemente originados em nossa galáxia. Além disso, o espectro de energia observado para a componente extragaláctica é consistente com o esperado para uma distribuição cosmológica de fontes de prótons:

$$
d N_{R C} / d E_{R C} \propto E_{R C}^{-2}
$$

O limite superior para o fluxo de neutrinos de altas energias é determinado a partir das reações entre prótons e o campo de radiação das fontes:

$$
\begin{aligned}
& p+\gamma \rightarrow \pi^{ \pm}+\mathrm{X} \\
& \leftrightarrow \mu \nu_{\mu} \\
& \quad \leftrightarrow e \nu_{e} \nu_{\mu}
\end{aligned}
$$

onde os píons carregados decaem produzindo neutrinos. Este limite assume que as fontes são opticamente "finas", ou seja, não são muito maiores do que o livre caminho médio desta reação. Com isso a maior parte dos prótons escapa da fonte, sendo que apenas uma pequena parcela deles interage dentro da mesma. Neste caso o espectro de neutrinos é dado por [11, 12]:

$$
\left(\frac{d N}{d E}\right)_{\mathrm{WB}}=\frac{(1-4) \times 10^{-8}}{E^{2}} \mathrm{GeV} \mathrm{cm}^{-2} \mathrm{~s}^{-1} \mathrm{sr}^{-1}
$$

onde o intervalo dado para os coeficientes depende da evolução cosmológica da fonte. Como mencionado anteriormente, em nosso trabalho, assumimos que o fluxo de neutrinos é dado pelo limite superior (limite WB) desta 
equação.

Podemos notar a partir das interações 2.2 que a proporção entre $\nu_{\mu}$ e $\nu_{e}$ produzidos é $2: 1$. Veremos mais adiante que a produção das NLSPs que buscamos em nosso trabalho independe do sabor do neutrino que interage na Terra. Desta forma, incluímos os $\nu_{e}$ que são produzidos conjuntamente com os $\nu_{\mu}$ no nosso fluxo inicial de neutrinos.

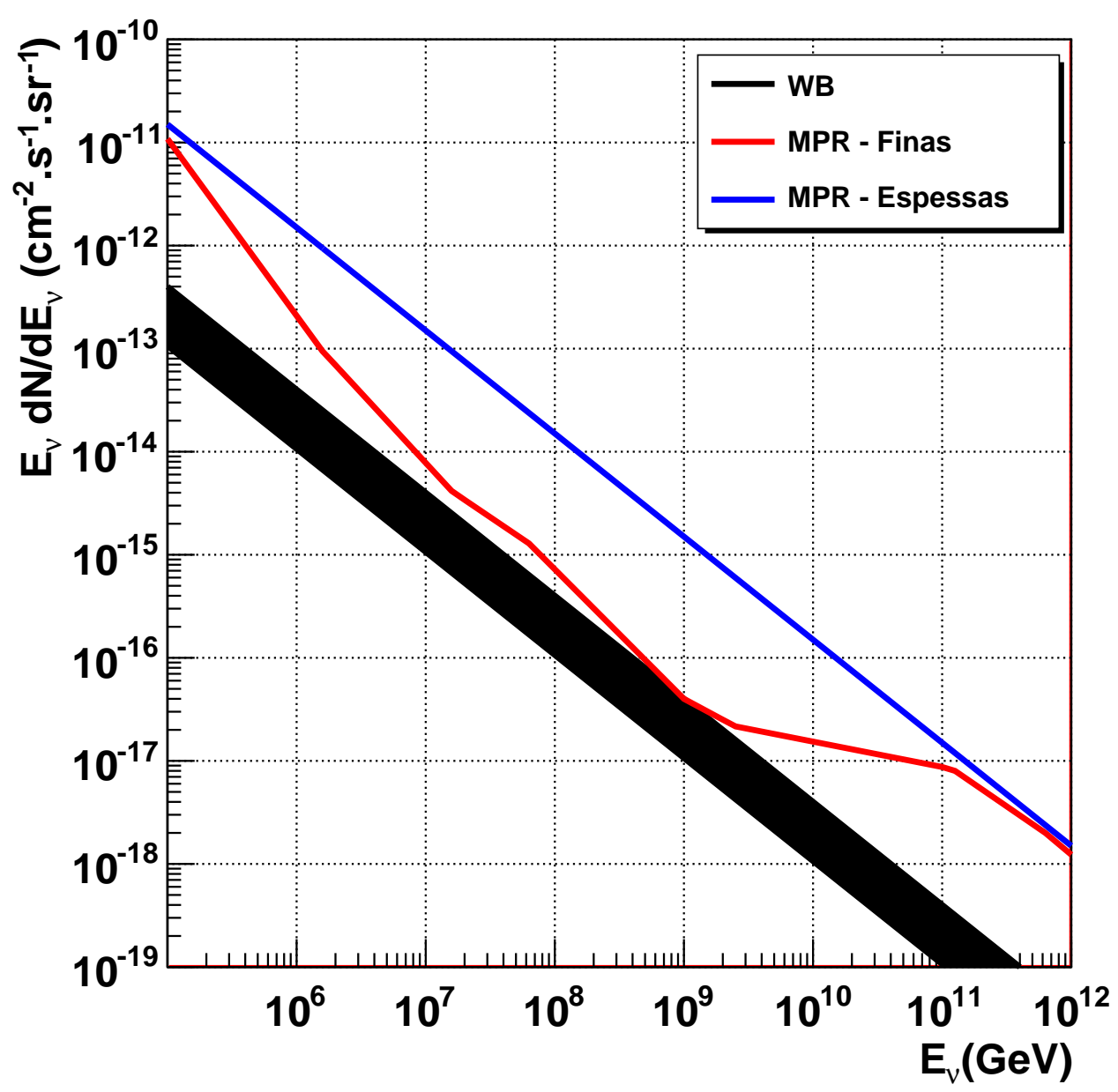

Figura 2.1: A área em preto representa o limite de Waxman e Bahcall (WB) desde o limite considerando evolução cosmológica (limite inferior) até o limite não considerando evolução cosmológica (limite superior). O limite de Mannheim, Protheroe e Rachen (MPR) para fontes opticamente "finas" é representado pela linha vermelha e no caso de fontes "espessas", pela linha azul. 


\subsection{Seção de choque neutrino-núcleon: Produção de Léptons}

Um parâmetro muito importante na determinação da taxa de NLSPs no telescópio de neutrinos, relativo à interação neutrino-núcleon, é a seção de choque inclusiva de corrente carregada, onde o lépton de mesmo sabor do neutrino é produzido. Aqui reproduzimos o cálculo da seção de choque [14] para a produção de léptons, e em seguida, na seção 2.3, determinamos a seção de choque para produção de NLSPs. A escala de energia considerada neste trabalho é definida a partir do limiar de energia para a produção de NLSPs $\left(E \gtrsim 10^{5} \mathrm{GeV}\right)$. Nesta escala os cálculos a seguir são válidos para todos os léptons. Na referência [15] são apresentados os cálculos explicitamente para o neutrino do tau e também são feitas comparações com os resultados para os neutrinos do múon. Para energias acima de $10^{3} \mathrm{GeV}$ as seções de choque para estes léptons são muito próximas.

A reação considerada é a seguinte:

$$
\nu_{l} N \rightarrow l^{ \pm}+X
$$

sendo a seção de choque diferencial dada por [14]:

$$
\frac{d^{2} \sigma_{\nu}}{d x d y}=\frac{2 G_{F}^{2} M E_{\nu}}{\pi} \frac{M_{W}^{2}}{Q^{2}+M_{W}^{2}}\left[x q\left(x, Q^{2}\right)+x \bar{q}\left(x, Q^{2}\right)\left(1-y^{2}\right)\right]
$$

neste caso, $x=Q^{2} / 2 M \nu$ é a fração de momento dos pártons; $y$ é a fração de energia perdida, dada por:

$$
y=\frac{E_{\nu}-E_{l}}{E_{\nu}}
$$

$M$ é a massa do núcleon; $G_{F}$ é a constante de Fermi; $M_{W}$ é a massa do bóson $W, Q$ é o momento transferido e as funções $q$ e $\bar{q}$ são dadas por:

$$
\begin{array}{r}
q\left(x, Q^{2}\right)=\frac{u_{v}\left(x, Q^{2}\right)+d_{v}\left(x, Q^{2}\right)}{2}+\frac{u_{s}\left(x, Q^{2}\right)+d_{s}\left(x, Q^{2}\right)}{2}+ \\
+s_{s}\left(x, Q^{2}\right)+b_{s}\left(x, Q^{2}\right)
\end{array}
$$




$$
\bar{q}\left(x, Q^{2}\right)=\frac{u_{s}\left(x, Q^{2}\right)+d_{s}\left(x, Q^{2}\right)}{2}+c_{s}\left(x, Q^{2}\right)+t_{s}\left(x, Q^{2}\right)
$$

Nas equações 2.7 e 2.8, $u, d, s, c, b$ e $t$ representam, respectivamente, as distribuições partônicas dos quarks up, down, strange, charm, bottom e top. Os índices $s$ e $v$ representam os quarks do mar e os de valência, respectivamente. Usando $Q^{2}$ em função de $x$ e $y$, é possível calcular a seção de choque $\sigma_{\nu}$ usando a seguinte igualdade:

$$
\begin{array}{r}
\sigma_{\nu}^{l}\left(E_{\nu}\right)=\int_{0}^{1} \int_{0}^{1} \frac{2 G_{F}^{2} M E_{\nu}}{\pi} \frac{M_{W}^{2}}{2 M E_{\nu} x y+M_{W}^{2}}\left[x q\left(x, Q^{2}\right)+\right. \\
\left.+x \bar{q}\left(x, Q^{2}\right)\left(1-y^{2}\right)\right] d x d y
\end{array}
$$

Para o cálculo numérico desta integral foi utilizada a biblioteca pdflib do pacote CERNLIB [16].

Quando os valores de $x$ são menores que $x_{M I N}=10^{-5}$ é utilizada uma aproximação para valores pequenos de fração de momento partônico [14]:

$$
x q_{s}\left(x, Q^{2}\right)=C\left(Q^{2}\right) \sqrt{\frac{2\left(\xi-\xi_{0}\right)}{\rho}} \exp \left\{\sqrt{2 \rho\left(\xi-\xi_{0}\right)}\right\}
$$

Para que haja continuidade na fronteira de $X_{M I N}, C\left(Q^{2}\right)$ foi definido da seguinte maneira:

$$
C\left(Q^{2}\right)=\frac{q_{s}\left(x \geq x_{M I N}\right)}{\sqrt{\frac{2\left(\xi-\xi_{0}\right)}{\rho_{M I N}}} \exp \left\{\sqrt{2 \rho_{M I N}\left(\xi-\xi_{0}\right)}\right\}},
$$

com $\rho_{M I N}=\left(8 N_{c} / b_{0}\right) \ln \left(1 / x_{M I N}\right)$, em que $N_{c}$ é o número de cores; $\xi(Q)=$ $\ln \left(\ln \left(Q^{2} / \Lambda^{2}\right)\right) ; b_{0}=\left(11 N_{c}-n_{f}\right) / 3$, com $n_{f}$ sendo o número de sabores.

O gráfico obtido para a seção de choque $\sigma_{\nu}$ é apresentado na Figura 2.2. Ele é compatível com os obtidos em [7] e [17]. A parametrização desta seção de choque é descrita no apêndice B.1. 


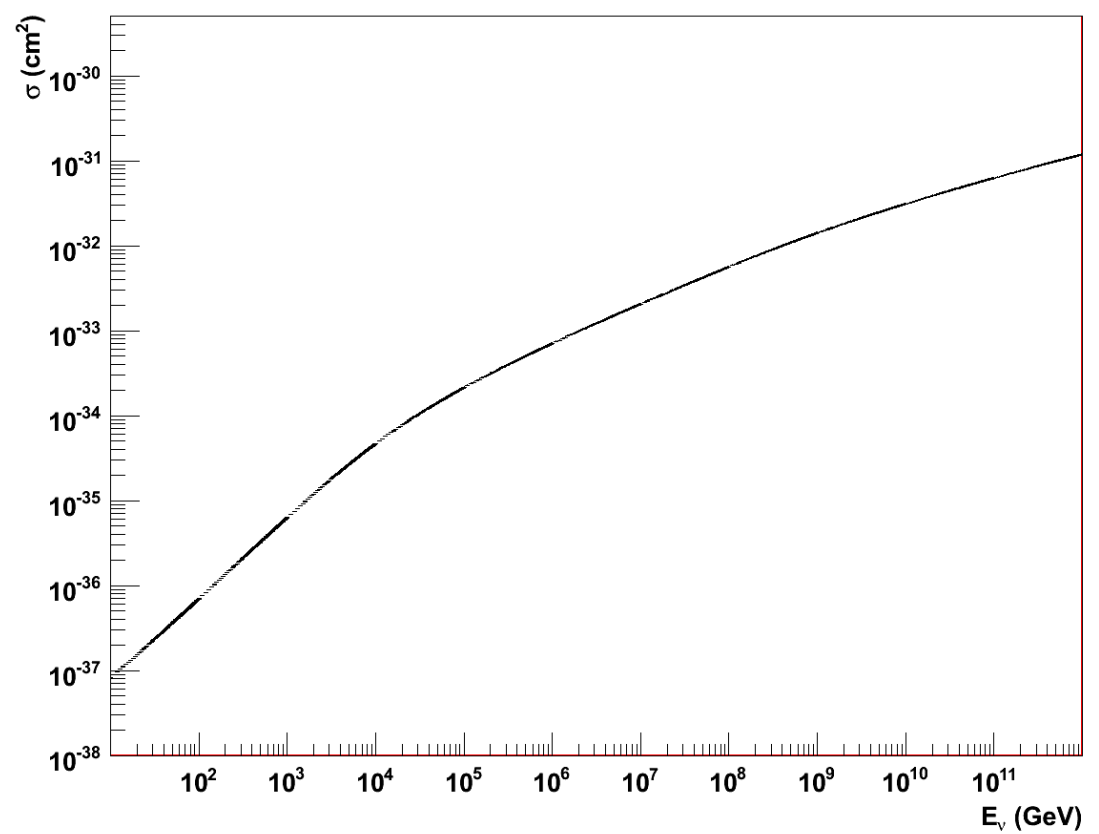

Figura 2.2: Seção de choque inclusiva de corrente carrega da interação neutrinonúcleon para a produção de léptons.

\subsection{Seção de choque neutrino-núcleon Produção de NLSPs}

Os processos supersimétricos para a produção de NLSPs, a partir de interações de neutrinos, são análogos aos de corrente carregada para léptons $[6]$ :

$$
\nu N \rightarrow \tilde{l}_{L} \tilde{q}
$$

Neste caso temos a produção de um slépton de mão esquerda e um squark. Eles são produzidos via canal $t$ por meio da troca de um gaugino, que neste caso é um chargino $(\tilde{W})$, pois a mistura com higgsinos no setor do gaugino é desprezada. O limiar de energia necessário para este processo ocorrer $(E \gtrsim$ $10^{5} \mathrm{GeV}$ ) é dado pelas massas dos $\tilde{l}_{L}$ e $\tilde{q}$. O $\tilde{l}_{L}$ e o $\tilde{q}$ produzidos prontamente decaem em uma cadeia de reações que sempre produzem no final dois sléptons de mão direita carregados $\left(\tilde{l}_{R}\right)$ mais partículas do Modelo Padrão. Este $\tilde{l}_{R}$ é 
a segunda partícula supersimétrica mais leve (NLSP), tipicamente o stau de mão direita $\left(\tilde{\tau}_{R}\right)$. As NLSPs são produzidas com aproximadamente $1 / 6$ da energia do neutrino $[6,7]$.

Na Figura 2.3 (obtida de [7]) são apresentados os diagramas das contribuições dominantes para seção de choque correspondente à reação em questão.

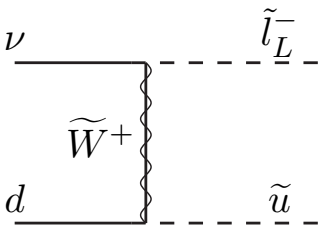

(a)

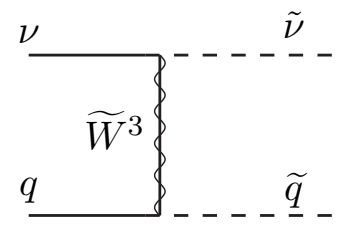

(c)

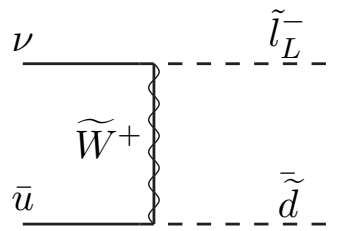

(b)

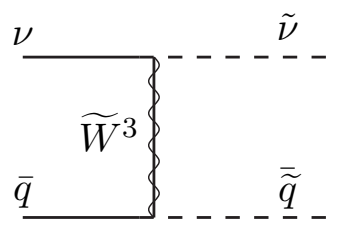

(d)

Figura 2.3: Diagramas de Feynman para a produção de partículas supersimétricas por meio de interações neutrino-núcleon. (a) Interação mão esquerda mão esquerda; (b) interação mão esquerda mão direita; (c) e (d) correspondem à corrente neutra. Há diagramas análogos para anti-neutrinos assim como para quarks iniciais do tipo strange e charm.

O neutrino, que é de mão esquerda, pode interagir tanto com um quark de mão esquerda do tipo down quanto com um anti-quark de mão direita do tipo up. Assim, temos duas contribuições para a seção de choque neutrino-núcleon partônica [6, 7], correspondentes às interações apresentadas nas Figuras 2.3a e 2.3b:

$$
\begin{gathered}
\frac{d \sigma^{(a)}}{d t}=\frac{\pi \alpha^{2}}{2 \sin ^{4} \theta_{W}} \frac{M_{\tilde{W}}^{2}}{s\left(t-M_{\tilde{W}}^{2}\right)^{2}} \\
\frac{d \sigma^{(b)}}{d t}=\frac{\pi \alpha^{2}}{2 \sin ^{4} \theta_{W}} \frac{\left(t u-m_{\tilde{l}_{L}}^{2} m_{\tilde{q}}^{2}\right)}{s^{2}\left(t-M_{\tilde{W}}^{2}\right)^{2}}
\end{gathered}
$$


em que $s, t$ e $u$ são as variáveis de Mandelstam. $M_{\tilde{W}}, m_{\tilde{l}_{L}}$ e $m_{\tilde{q}}$ são respectivamente as massas do chargino, do slépton de mão esquerda e do squark. São adotados valores bem representativos para essas três massas nos cenários levados em consideração: $250 \mathrm{GeV}$ para o chargino, $250 \mathrm{GeV}$ para o slépton de mão esquerda e três possíveis valores para a massa do squark: $300 \mathrm{GeV}$, $600 \mathrm{GeV}$ e $900 \mathrm{GeV}$.

Para efetuar o cálculo numérico desta seção de choque é necessário primeiramente escrever as variáveis de Mandelstam em função das variáveis partônicas utilizadas no pacote CERNLIB [16]. Neste caso, obtemos as variáveis de Mandelstam em função de $y$, que é a fração de energia perdida.

Também é necessário multiplicar as equações 2.13 e 2.14 por suas distribuições partônicas correspondentes. Por exemplo, no caso da equação 2.13, quando temos o caso de um neutrino interagindo com um quark de mão esquerda do tipo down, deve-se multiplicá-la por:

$$
f_{L L}^{(a)}=\frac{q+s t r+b o t}{x}
$$

em que $x$ é a fração de momento dos pártons. $q$, str e bot são as distribuições partônicas para quarks do tipo down das três diferentes famílias. Quando a interação do neutrino for com o anti-quark de mão direita do tipo up, que é o caso da equação 2.14, devemos multiplicá-la por:

$$
f_{L R}^{(b)}=\frac{\bar{q}+c h m+t o p}{x}
$$

Da mesma forma como nos dois exemplos anteriores, devem ser considerados todos os casos do neutrino e antineutrino para ao final obter a seção de choque total.

É necessário integrar a seção de choque diferencial nas variáveis $x$ e $y$. Os limites de integração para $x$ vão de $X_{M I N}=\left(m_{\tilde{l}_{L}}+m_{\tilde{q}}\right)^{2} /\left(2 M_{p} E_{\nu}\right)$ até 1 e para $y$ são de 0 até 1 . Para valores pequenos de $x$ (menor do que $X_{M I N}=$ $10^{-5}$ ) procedemos da mesma forma que no caso dos léptons.

O gráfico obtido para a seção de choque de produção das partículas supersimétricas é apresentado na Figura 2.4. As curvas azul, vermelha e verde representam os casos em que a massa do squark produzido é de $300 \mathrm{GeV}, 600$ $\mathrm{GeV}$ e $900 \mathrm{GeV}$ respectivamente. É apresentada também a seção de choque 
para a produção de léptons, em preto. Vale observar que as curvas obtidas aqui são compatíveis com as obtidas em [6,7]. As parametrizações das curvas são descritas no apêndice B.2.

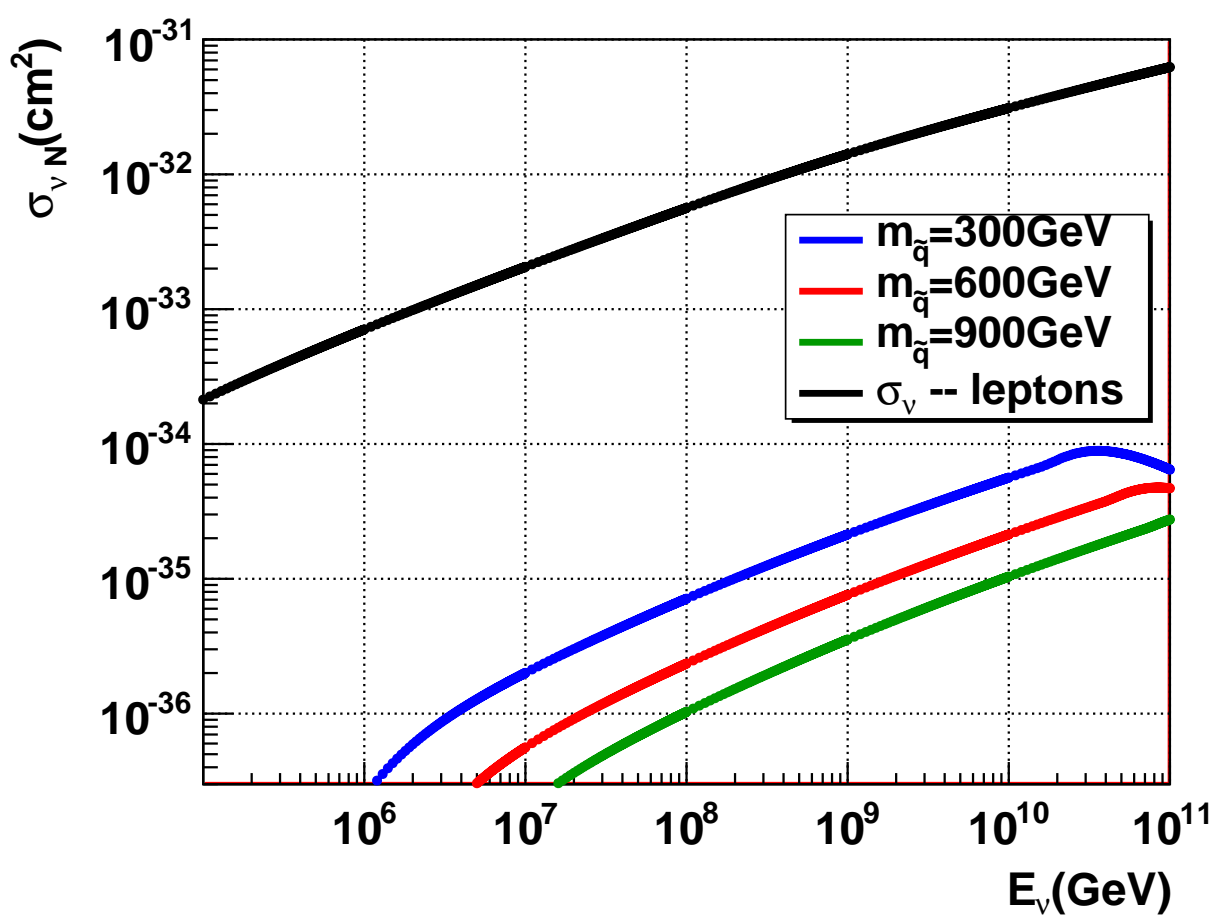

Figura 2.4: Seção de choque da interação neutrino-núcleon para a produção de partículas supersimétricas. São considerados três cenários para massa do squark produzido na interação neutrino-núcleon: $300 \mathrm{GeV}, 600 \mathrm{GeV}$ e $900 \mathrm{GeV}$. Estas curvas são compatíveis com [6]. Também é apresentada a curva da a seção de choque para produção de léptons em preto.

\subsection{Produção de NLSPs e léptons a partir da interação neutrino-núcleon}

A partir do limite WB temos uma estimativa do número de neutrinos que chegam à Terra em função da energia destas partículas. Com a seção de choque neutrino-núcleon quantificamos a interação entre o neutrino e os núcleons no interior do planeta, em função da energia dos neutrinos. Precisamos agora determinar a chance dos neutrinos incidentes interagirem no 
interior da Terra, que possui um perfil de densidade que muda em função do raio interno do planeta (seção 2.4.1). Para isto nos valemos da probabilidade de sobrevivência $\left(P_{s_{\nu}}\right)$ do neutrino, que depende da seção de choque inclusiva de corrente carregada neutrino-núcleon $\left(\sigma_{\nu}\right)$ e da densidade do meio no qual o neutrino se propaga $(\rho(x))[1]$ :

$$
P_{s_{\nu}}\left(E_{\nu}\right)=\exp \left(-\int \frac{\rho(x)}{M} \sigma_{\nu}\left(E_{\nu}\right) d x\right)
$$

A figura 2.5 ilustra curvas da probabilidade de sobrevivência do neutrino, relativas à produção de léptons $\left(P_{s_{\nu}}^{l}\right)$, em função do cosseno do ângulo de incidência do mesmo sobre a Terra, para seis valores de energia de incidência. Foi tomado um ponto fixo de referência a cerca de $2 \mathrm{~km}$ de profundidade (figura 2.8). As curvas apresentadas são compatíveis com as obtidas em [18]. Nota-se que a probabilidade de sobrevivência depende fortemente tanto da energia de incidência quanto do caminho a ser percorrido na Terra. Quanto maior a energia e quanto mais Terra há a ser percorrida, maior a chance do neutrino interagir. Aqui não levamos em conta a interação de corrente neutra. Esta age degradando a energia do neutrino, sem diminuir seu fluxo. Dado que a seção de choque é baixa, este efeito é desprezível.

A probabilidade do neutrino interagir é obtida diretamente da probabilidade de sobrevivência, sendo dada por:

$$
P_{i_{\nu}}\left(E_{\nu}\right)=1-P_{s_{\nu}}
$$

Para o caso da probabilidade do neutrino interagir relativa à produção de NLSPs $\left(P_{i_{\nu}}^{\tilde{\tau}}\left(E_{\nu}\right)\right)$, a seção de choque a ser levada em consideração para a interação neutrino-núcleon é a de produção de partículas supersimétricas $\left(\sigma_{\nu}^{\tilde{\tau}}\right)$, dada pelas equações 2.13 e 2.14 . 


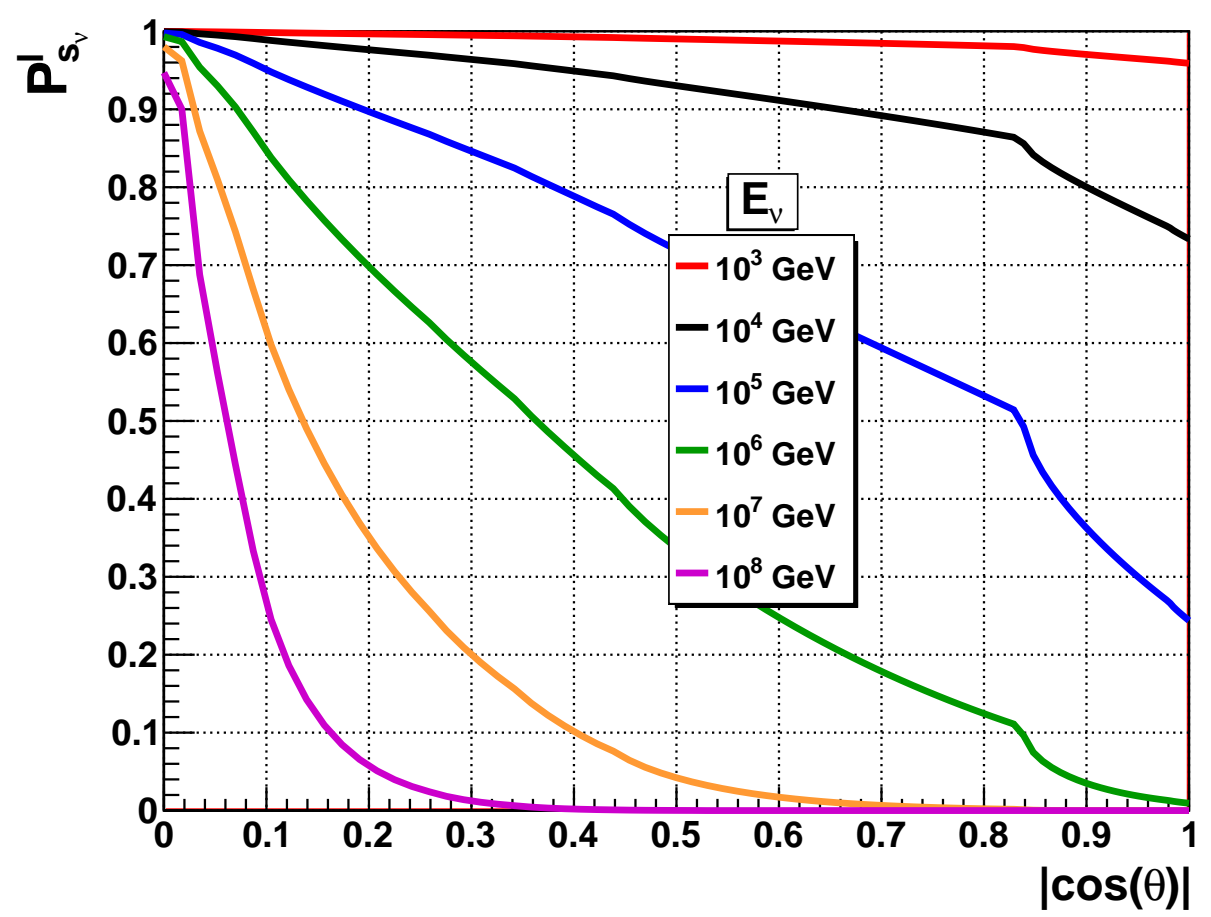

Figura 2.5: Probabilidade de sobrevivência do neutrino ao atravessar a Terra em função do cosseno do ângulo de incidência, para 6 valores de energia. Foi tomado um ponto de referência fixo a cerca de $2 \mathrm{~km}$ de profundidade.

Na figura 2.6 são apresentadas alguns exemplos de curvas da probabilidade de interação do neutrino devido à produção de NLSPs, em função do cosseno do ângulo de incidência do neutrino, para diferentes energias desta partícula. Similarmente à probabilidade de sobrevivência do neutrino para a produção de léptons, as curvas obtidas para a probabilidade de interação relativas à produção de NLSPs também apresentam uma forte dependência da energia do neutrino incidente, bem como da profundidade de Terra a ser percorrida. Porém no último caso a probabilidade é em geral, menor do que no primeiro caso, devido à supressão provocada pelas massas na seção de choque de produção de partículas supersimétricas. Em ambas as figuras a variação abrupta na probabilidade próximo a $\cos (\theta)=0,8$ é devida à variação da densidade do meio. 


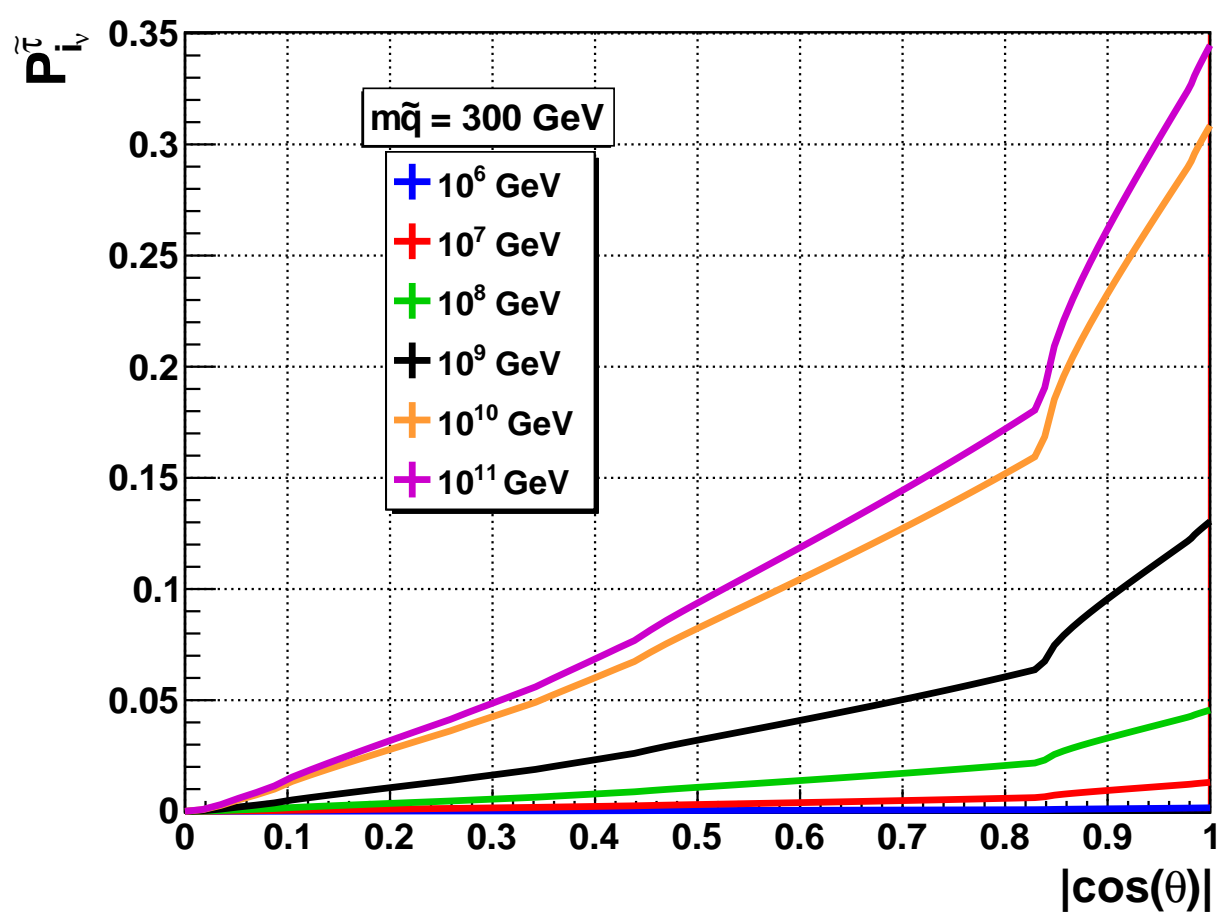

Figura 2.6: Probabilidade do neutrino interagir, produzindo um slépton, ao atravessar a Terra em função do cosseno do ângulo de incidência, para 6 valores de energia de incidência do neutrino. Foi tomado como alvo um ponto fixo na Terra, a cerca de $2 \mathrm{~km}$ de profundidade.

A probabilidade de produzir um slépton que atinja o detector, $P_{\tilde{\tau}_{d e t}}$, é dada por:

$$
P_{\tilde{\tau}_{\text {det }}}=P_{s_{\nu}}^{l}\left(E_{\nu}\right) \times P_{i_{\nu}}^{\tilde{\tau}}\left(E_{\nu}\right),
$$

representando a convolução entre a probabilidade de produção da NLSP e a probabilidade do neutrino sobreviver ${ }^{2}$ até o ponto a partir do qual uma NLSP produzida é capaz de chegar ao detector, ou seja, correspondente ao alcance máximo ${ }^{3}$ desta partícula. Este produto aparece como um peso probabilístico no processo de determinação da taxa de NLSPs no detector (seção 2.6). Para ilustrar o comportamento desta convolução, na figura 2.7 são apresentadas

\footnotetext{
${ }^{2}$ A probabilidade de sobrevivência do neutrino (equação 2.17) utiliza a seção de choque de produção de léptons.

${ }^{3} \mathrm{O}$ alcance máximo é a distância que a partícula pode percorrer antes de perder toda sua energia (seção 2.5.4).
} 
curvas para sete diferentes valores de energia de incidência do neutrino.

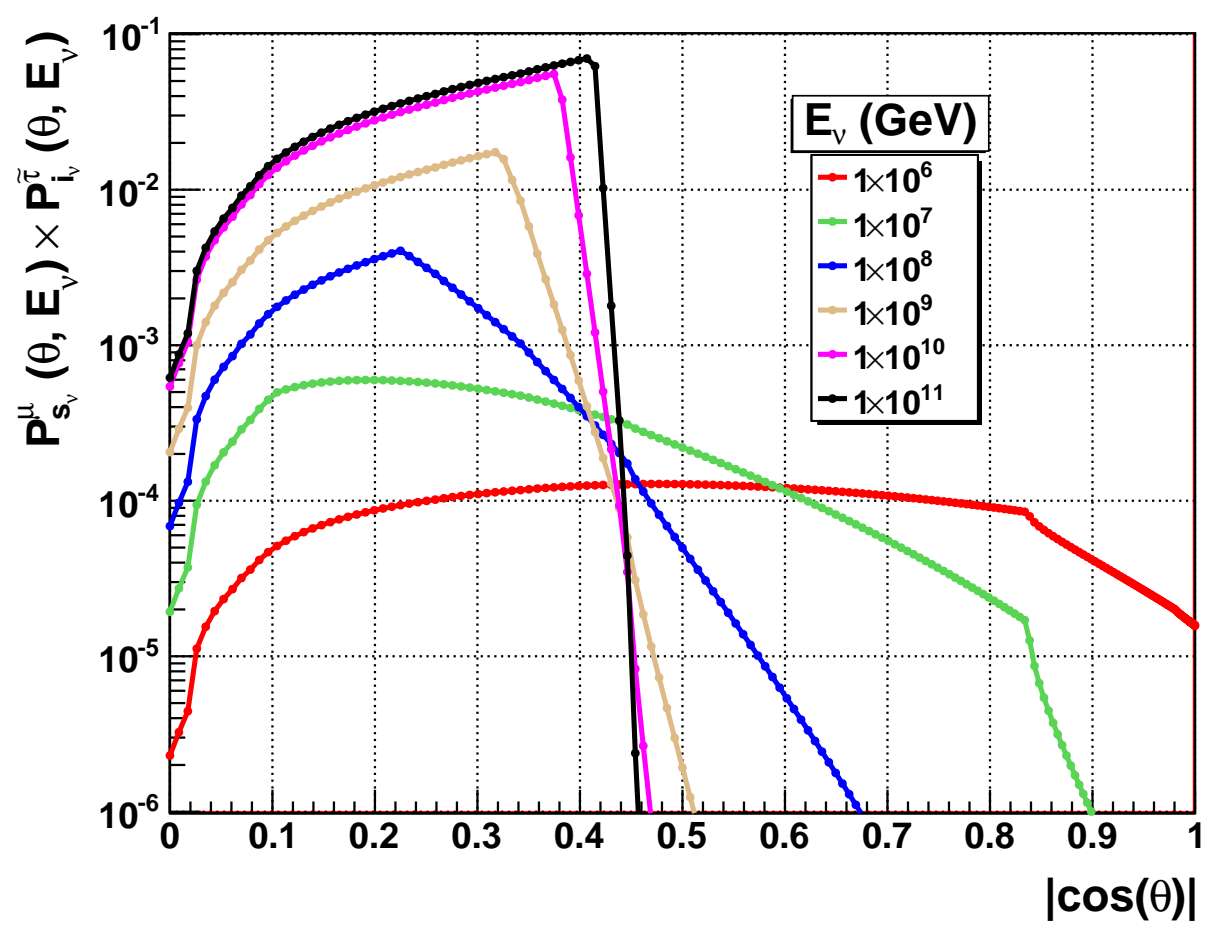

Figura 2.7: Probabilidade resultante da convolução das probabilidades de sobrevivência do neutrino (levando em conta a probabilidade de produzir múons), até o ponto em que o stau produzido pode chegar ao detector, e de interação do mesmo (produção de stau), em função do cosseno do ângulo de incidência, para 6 valores de energia de incidência do neutrino. Foi utilizado um ponto de referência fixo na Terra, a cerca de $2 \mathrm{~km}$ de profundidade.

\subsubsection{Perfil de densidade da Terra}

As probabilidades de interação de neutrinos com núcleons que compõem a Terra, bem como a degradação de energia dos léptons ou NLSPs produzidos, dependem da densidade do meio percorrido por estas partículas (equações 2.17, 2.18). A figura 2.8 ilustra a Terra aproximada por uma esfera e os parâmetros geométricos necessários para a descrição da trajetória das partículas simuladas. A profundidade do detector é indicada pela distância $a$ e o ângulo de incidência $\theta$ é medido no referencial do detector. A geometria do detector não é levada em consideração, pois estamos interessados na taxa de eventos por unidade de área. 


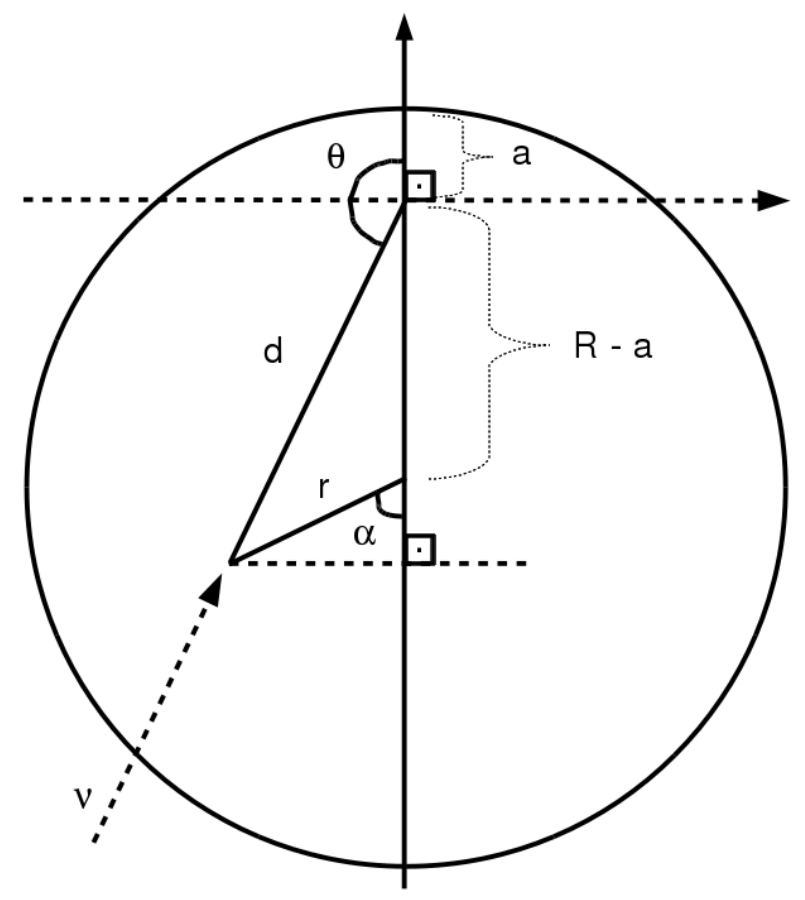

Figura 2.8: Esquema da Terra com os parâmetros utilizados para a determinação do seu perfil de densidade. A distância $a$ representa a profundidade do detector.

Adotamos o perfil de densidade descrito pela equação 2.20 [14], onde a Terra é dividida em camadas esféricas concêntricas, sendo $r$ (em $\mathrm{km}$ ) a distância ao centro da Terra, $x \equiv r / R$ e o raio da Terra dado por $R=6371$ $\mathrm{km}$ :

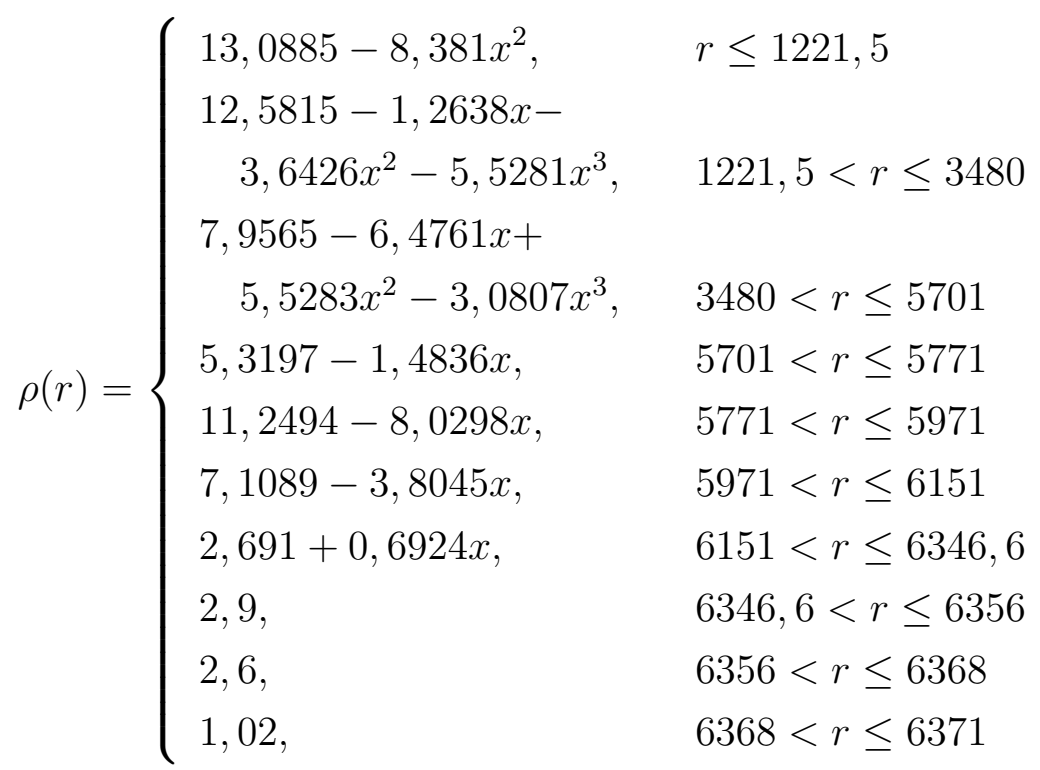


A dependência do ângulo $\theta$ na densidade aparece explicitamente quando relacionamos o raio $r$ com os parâmetros apresentados na figura 2.8: $r$ é dado em função de $\theta, d, R$ e $a$ da seguinte maneira:

$$
r=\sqrt{d^{2}+(R-a)^{2}+2(R-a) d \cos (\theta)}
$$

Nas simulações, é necessário determinar $d$ analiticamente apenas inicialmente, quando $r=R$ na figura 2.8. Neste caso, $d$ é dado por:

$$
d=-(R-a) \cos (\theta)+\sqrt{R^{2}-(R-a)^{2} \sin ^{2}(\theta)}
$$

A Figura 2.9 ilustra o perfil de densidade encontrado por uma partícula viajando pela Terra em função da distância percorrida, que é medida da superfície até o detector a aproximadamente $2 \mathrm{~km}$ de profundidade, para vários ângulos de incidência. Quando o ângulo vale 180 graus, a distância total é o diâmetro da Terra subtraído do valor da profundidade do detector.

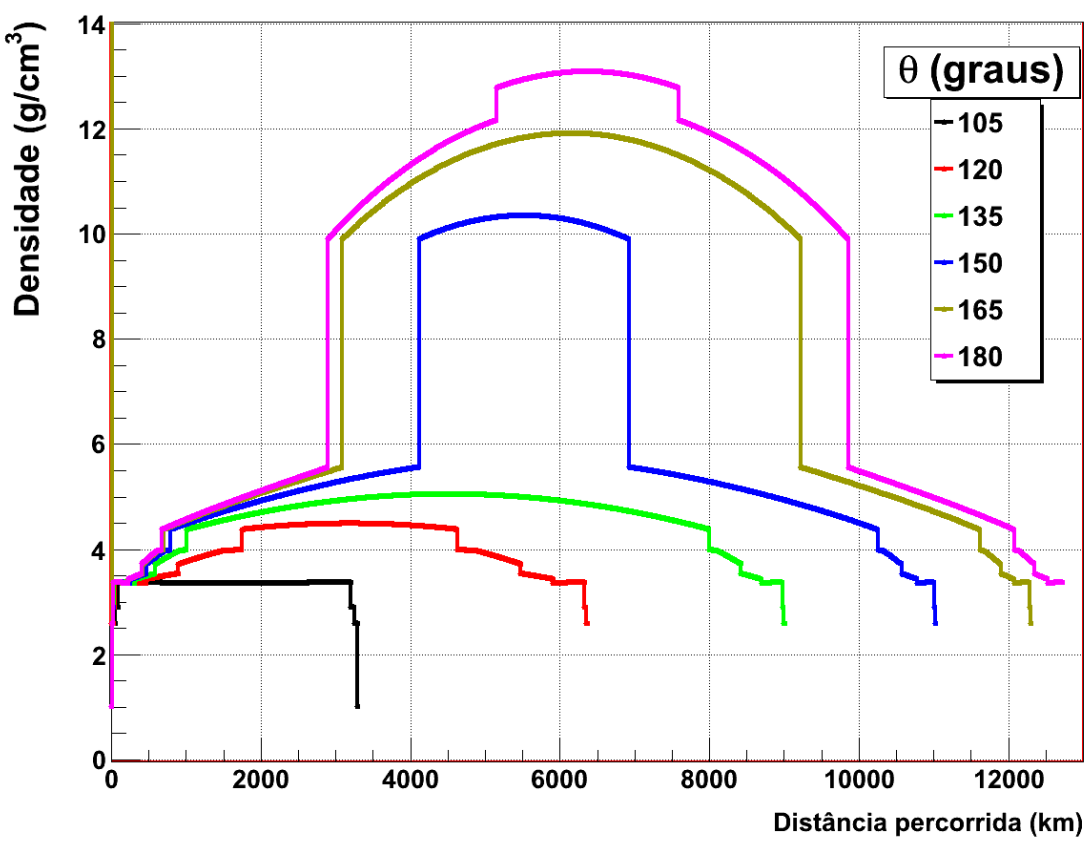

Figura 2.9: Perfil de densidade da Terra encontrado por uma partícula que incide sobre o planeta em diferentes ângulos. A distância percorrida é medida a partir da superfície da Terra até o detector, a aproximadamente $2 \mathrm{~km}$ de profundidade. 


\subsection{Perda de energia de (s)léptons na Terra}

Uma vez produzidos por meio de interações de neutrinos com a Terra, os léptons e as NLSPs, por serem carregados, perdem energia tanto por ionização como por radiação ao longo de sua trajetória no interior do planeta. Levamos este efeito em consideração para determinar o espectro de energia com o qual atingem o detector e também a fração de partículas que perdem toda energia no trajeto.

Nesta seção reproduzimos os cálculos [7] das perdas médias de energia dos múons, taus e staus. Também determinamos o alcance máximo destas partículas. Este parâmetro define a distância máxima em que elas podem ser produzidas em relação ao detector, de tal forma que o atinjam com energia suficiente para que possam ser observadas.

A perda de energia média, por unidade de comprimento, das partículas carregadas é dada por [19]:

$$
-\frac{d E}{d x}=a(E)+b(E) E
$$

sendo $a(E)$ a contribuição devido à perda por ionização e $b(E)$, por radiação. Esta última é a contribuição dominante para altas energias, com três processos principais: perda por bremsstrahlung, por produção de pares e por interação fotonuclear, discutidas em detalhes nas seções 2.5.1 a 2.5.3. A dependência da perda de energia em relação à ionização é pequena comparada à por radiação, podendo ser considerada constante: $\sim 2 \times 10^{-3} \mathrm{GeV} \cdot \mathrm{cm}^{2} \cdot \mathrm{g}^{-1}$ $[7]$.

Nas Figuras 2.10 a 2.12 são apresentados os resultados obtidos para as três contribuições referentes à perda de energia por radiação citadas, respectivamente para múons, taus e sléptons. Para este último adotamos sempre uma massa de repouso de $150 \mathrm{GeV}$. Nota-se que a perda de energia diminui significativamente para as partículas mais pesadas, principalmente quando se comparam os sléptons com os múons, como ficará claro nas seções a diante. Vale observar que estes resultados são compatíveis com os de [7]. 


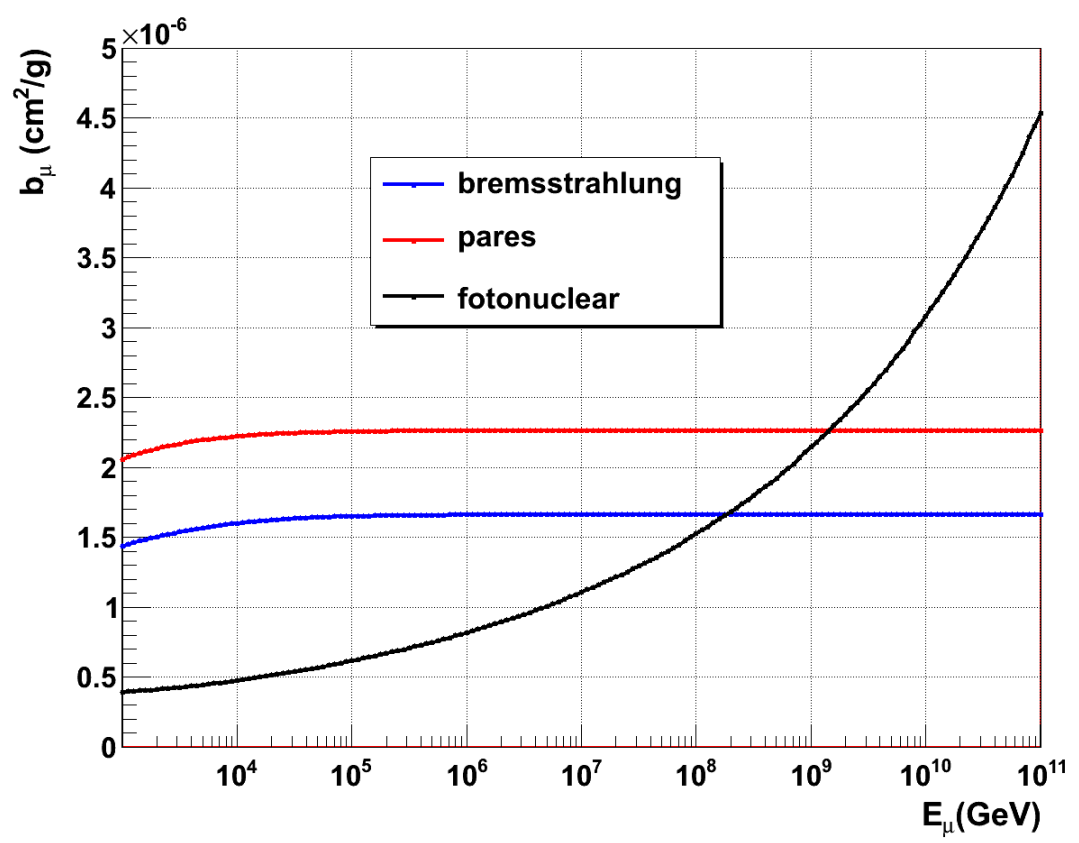

Figura 2.10: Perda de energia média (por radiação) de múons em função de sua energia. São apresentadas as contribuições relevantes: bremsstrahlung, produção de pares e interação fotonuclear.

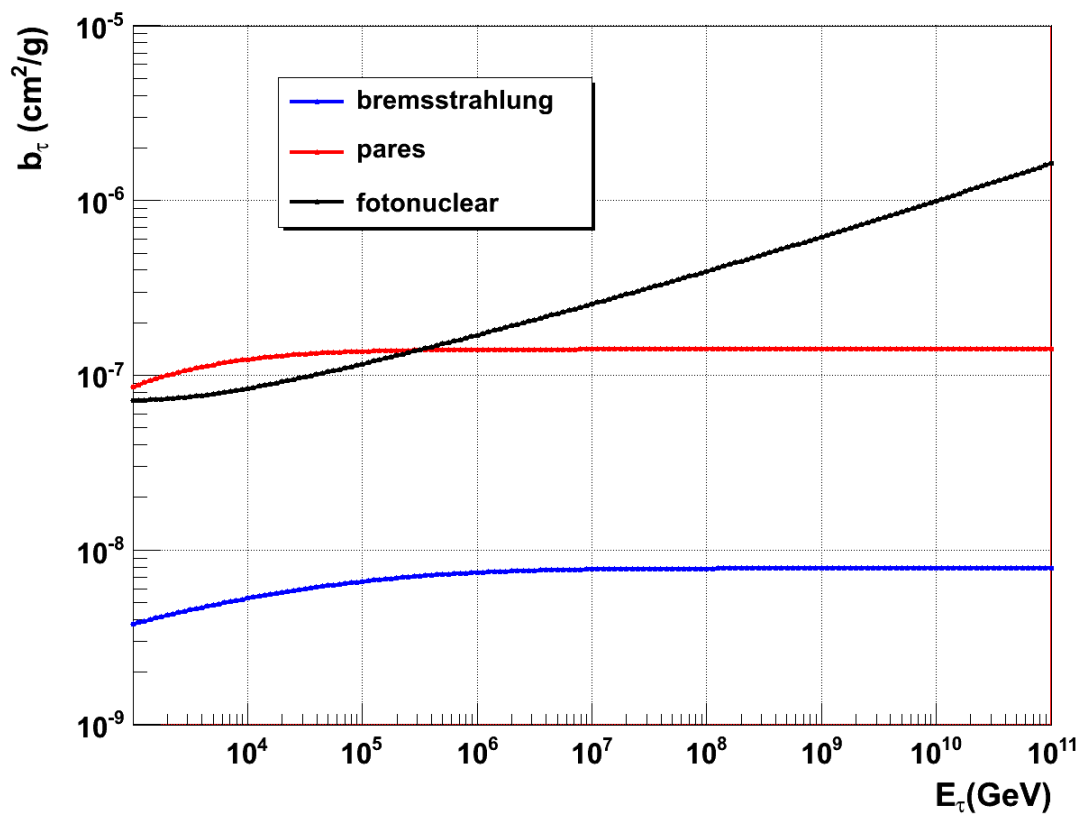

Figura 2.11: Perda de energia (por radiação) do tau em função de sua energia. São apresentadas as contribuições relevantes: bremsstrahlung, produção de pares e interação fotonuclear. 


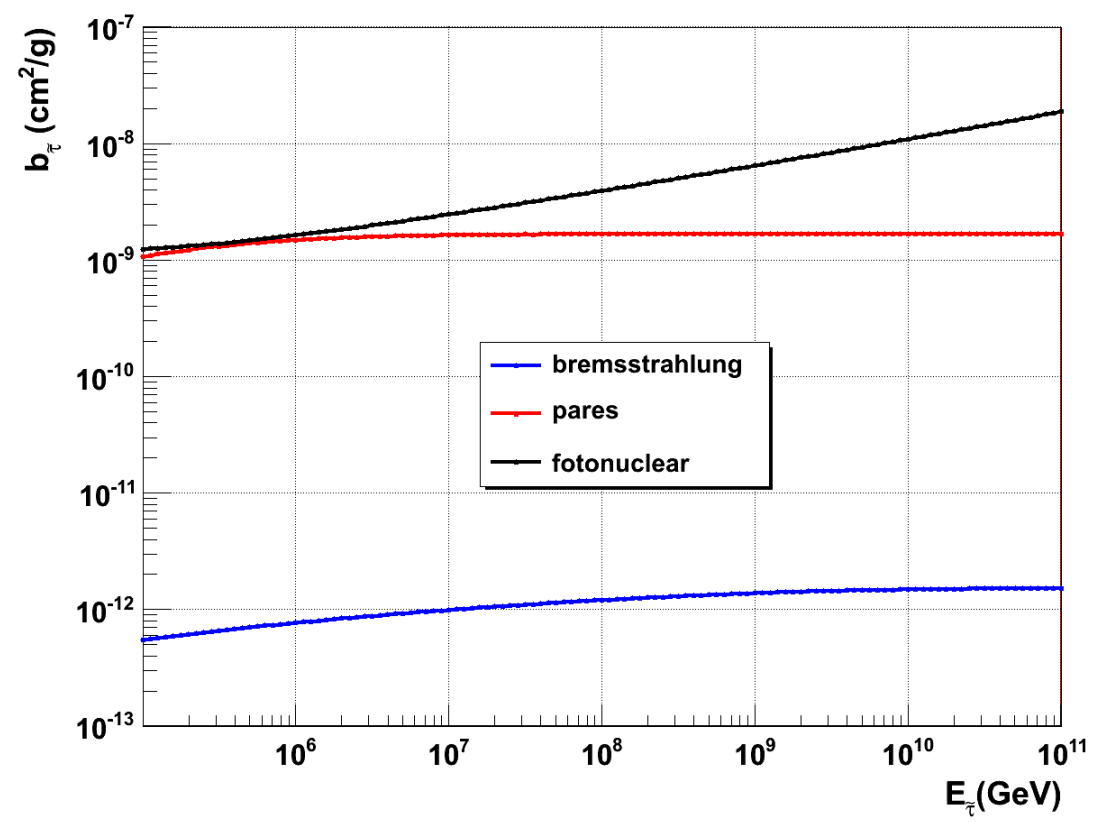

Figura 2.12: Perda de energia média (por radiação) do slépton em função de sua energia. São apresentadas as contribuições relevantes: bremsstrahlung, produção de pares e interação fotonuclear.

Para compararmos diretamente a perda de energia por radiação das três partículas em questão, são apresentadas na figura 2.13 as somas das três principais contribuições. Observa-se que a perda de energia do tau é cerca de uma ordem de grandeza menor do que a do múon. A do stau, por sua vez, é cerca de duas ordens de grandeza menor do que a do tau. Por isso há um ganho em volume muito grande em favor das NLSPs, que podem percorrer distâncias muito maiores antes de perder toda a sua energia.

Para uma performance mais satisfatória nas simulações, a soma das contribuições para a perda de energia por radiação foi parametrizada, tanto para múons e taus, quanto para sléptons. Este procedimento torna a simulação muito mais rápida, pois os cálculos de $b(E)$ demandam muito processamento. Estas parametrizações são descritas no apêndice B.3.

Nas subseções a seguir são apresentados em detalhes os cálculos para a perda de energia dos (s)léptons pelas três perdas por radiação citadas. Foram tomados como referência os passos apresentados em [7]. 


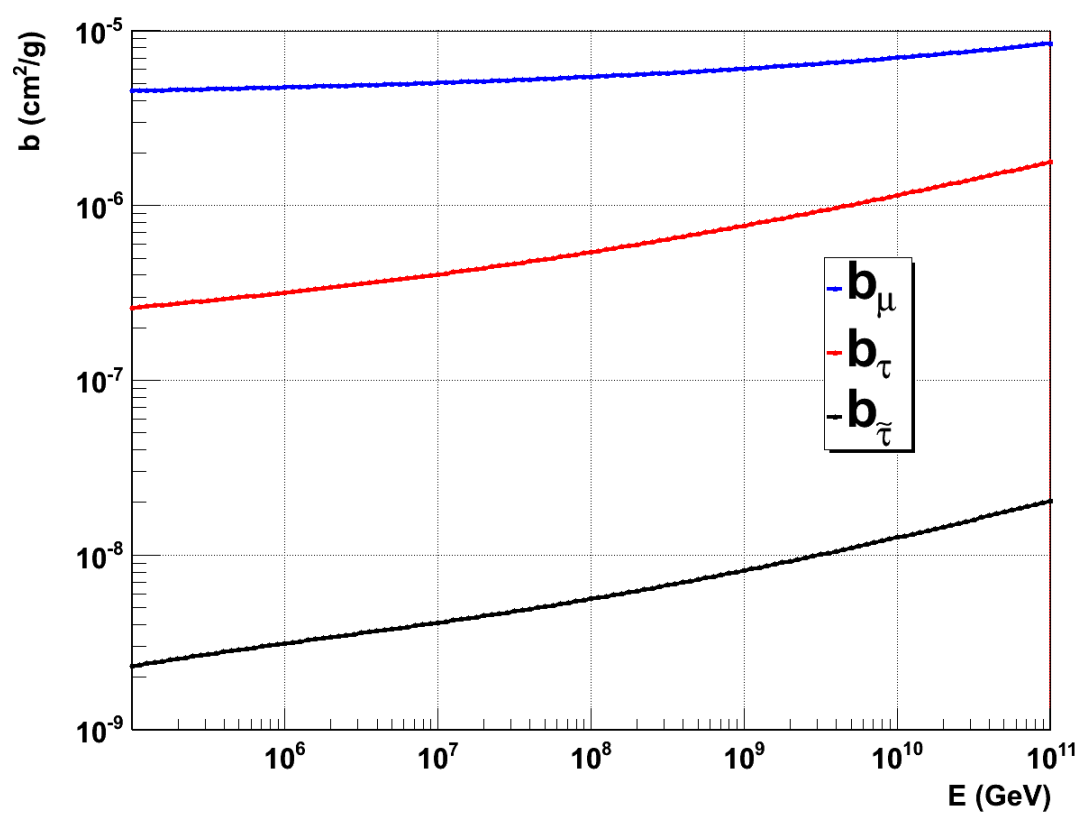

Figura 2.13: Perda total de energia por radiação do múon, tau e slépton em função de suas energias. São apresentadas às somas das três contribuições relevantes: bremsstrahlung, produção de pares e interação fotonuclear.

\subsubsection{Bremsstrahlung}

Bremsstrahlung [20, 21] consiste na radiação eletromagnética produzida pela aceleração de uma partícula carregada quando defletida por outra partícula carregada. Tal efeito gera um espectro de radiação contínuo. No caso das partículas consideradas neste trabalho (múons, taus e staus) e das respectivas escalas de energia, é necessário levar em conta o efeito de screening nuclear [22], que é dominante.

A contribuição na perda de energia devido à radiação bremsstrahlung é dada por:

$$
b_{b r e m}(E)=\frac{N_{A}}{A} \int_{y_{\min }}^{y_{\max }} y \frac{d \sigma}{d y} d y
$$

onde $N_{A}$ é o número de Avogadro e $A$, o número atômico do alvo. A transferência de energia $y$ é dada por: 


$$
y=\frac{E-E^{\prime}}{E},
$$

onde $E$ é a energia da partícula carregada antes da emissão da radiação e $E^{\prime}$ é sua energia após ser defletida, no referencial da partícula alvo. A seção de choque diferencial $\frac{d \sigma}{d y}$ é dada por [22]:

$$
\frac{d \sigma}{d y}=\alpha\left(2 Z \frac{m_{e}}{m_{l}} r_{e}\right)^{2}\left(\frac{4}{3}-\frac{4}{3} y+y^{2}\right) \frac{\Phi(\delta)}{y}
$$

em que $m_{l}$ é a massa do (s)lépton, $m_{e}$ é a massa do elétron, $r_{e}$ é o raio clássico do elétron, $\alpha$ é a constante de estrutura fina (1/137), $Z$ é o número atômico do alvo e $\Phi(\delta)$ é dado por:

$$
\Phi(\delta)=\ln \left(\frac{B m_{l} Z^{-1 / 3} / m_{e}}{1+\delta \sqrt{e} B Z^{-1 / 3} / m_{e}}\right)-\ln \left(\frac{D_{n}}{1+\delta\left(D_{n} \sqrt{e}-2\right) / m_{l}}\right)
$$

$\operatorname{com} \delta$ definido da seguinte maneira:

$$
\delta=\frac{m_{l}^{2}}{2 E(1-y)}
$$

$D_{n}$ é dado por:

$$
D_{n}=1.54 A^{0,27}
$$

$e$ é o número de Euler e $B=182,7$. Os limites de integração para $y$ são $y_{\min }=0$ e $y_{\max }=1-(3 / 4) \sqrt{e} Z^{1 / 3}\left(m_{l} / E\right)$.

Na equação 2.26 o fator $Z^{2}$ pode ser substituído por $Z(Z+1)$ com o intuito de aproximar o efeito de bremsstrahlung induzido por elétrons atômicos, que é desprezível.

Estas equações mostram que o efeito de bremsstrahlung diminui para massas maiores, o que pode ser visualizado nas figuras 2.10 a 2.12.

\subsubsection{Produção de Pares}

Este efeito é devido à perda de energia por meio da produção direta de pares $e^{+} e^{-}$[22] e é dada por: 


$$
b_{p a r}(E)=\frac{N_{A}}{A} \int_{y_{\min }}^{y_{\max }} \int_{0}^{\rho_{\max }} y \frac{d^{2} \sigma}{d y d \rho} d \rho d y,
$$

onde $y$ é definido pela equação 2.25 e $\rho$ é o parâmetro de assimetria dado por [17]:

$$
\rho=\frac{E^{+}-E^{-}}{E^{+}+E^{-}}
$$

sendo $E^{+}$e $E^{-}$respectivamente as energias do elétron e do pósitron do par produzido.

Os limites de integração para $y$ são:

$$
\frac{4 m_{e}}{E} \leq y \leq 1-\frac{3}{4} \sqrt{e} Z^{1 / 3} \frac{m_{l}}{E}
$$

e para $\rho$ :

$$
0 \leq \rho \leq\left(1-\frac{6 m_{l}^{2}}{(1-y) E^{2}} \sqrt{1-\frac{4 m_{e}}{y E}}\right)
$$

A seção de choque diferencial $\frac{d^{2} \sigma}{d y d \rho}$ é dada por [23]:

$$
\frac{d^{2} \sigma}{d y d \rho}=\frac{2}{3 \pi} \alpha^{4}\left(Z \lambda_{e}\right)^{2} \frac{1-y}{y}\left(\phi_{e}+\frac{m_{e}^{2}}{m_{l}^{2}} \phi_{l}\right)
$$

em que $\lambda_{e}$ é o comprimento de onda Compton do elétron. Os campos $\phi_{e} \mathrm{e}$ $\phi_{l}$ são dados por $[22,17]$ :

$$
\begin{gathered}
\phi_{e}=\left\{\left[\left(2+\rho^{2}\right)(1+\beta)+\xi\left(3+\rho^{2}\right)\right] \ln \left(1+\frac{1}{\xi}\right)+\right. \\
\left.+\frac{1-\rho^{2}-\beta}{1+\xi}-\left(3+\rho^{2}\right)\right\} L_{e}, \\
\phi_{l}=\left\{\left[\left(1+\rho^{2}\right)\left(1+\frac{3}{2} \beta\right)-\frac{1}{\xi}(1+2 \beta)\left(1-\rho^{2}\right)\right] \ln (1+\xi)+\right. \\
\left.+\frac{\xi\left(1-\rho^{2}-\beta\right)}{1+\xi}+(1+2 \beta)\left(1-\rho^{2}\right)\right\} L_{l},
\end{gathered}
$$


com $L_{e}$ e $L_{l}$ nas equações 2.35 e 2.36 dados por:

$$
\begin{gathered}
L_{e}=\ln \left(\frac{R Z^{-1 / 3} \sqrt{(1+\xi)\left(1+Y_{e}\right)}}{1+\frac{2 m_{e} \sqrt{e} R Z^{-1 / 3}(1+\xi)\left(1+Y_{e}\right)}{y E\left(1-\rho^{2}\right)}}\right)- \\
\frac{1}{2} \ln \left[1+\left(\frac{3}{2} \frac{m_{e}}{m_{l}} Z^{1 / 3}\right)^{2}(1+\xi)\left(1+Y_{e}\right)\right], \\
L_{l}=\ln \left[\frac{R Z^{-2 / 3} \frac{2}{3} \frac{m_{l}}{m_{e}}}{1+\frac{2 m_{e} \sqrt{e} R Z^{-1 / 3}(1+\xi)\left(1+Y_{l}\right)}{y E\left(1-\rho^{2}\right)}}\right] .
\end{gathered}
$$

Nas equações 2.37 e $2.38, R$ é uma constante cujo valor é $R=189$. $Y_{e}$ e $Y_{l}$ são dados por:

$$
\begin{gathered}
Y_{e}=\frac{5-\rho^{2}+4 \beta\left(1+\rho^{2}\right)}{2(1+3 \beta) \ln (3+1 / \xi)-\rho^{2}-2 \beta\left(2-\rho^{2}\right)} \\
Y_{l}=\frac{4+\rho^{2}+3 \beta\left(1+\rho^{2}\right)}{\left(1+\rho^{2}\right)\left(\frac{3}{2}+2 \beta\right) \ln (3+\xi)+1-\frac{3}{2} \rho^{2}}
\end{gathered}
$$

Por fim, $\beta$ e $\xi$ são definidos como:

$$
\begin{gathered}
\beta=\frac{y^{2}}{2(1-y)} \\
\xi=\left(\frac{m_{l} y}{2 m_{e}}\right)^{2} \frac{\left(1-\rho^{2}\right)}{(1-y)} .
\end{gathered}
$$

Aqui também a perda de energia é maior para massas menores, como pode ser notado nas figuras 2.10 a 2.12 .

\subsubsection{Interação fotonuclear}

A perda de energia por interação fotonuclear é devida à interação forte que ocorre quando as partículas se propagam próximas a núcleons. Este efeito é mais significativo para as partículas mais massivas e para energias mais altas. Como pode ser visto nas figuras 2.10 a 2.12 , no caso do tau 
esta perda de energia domina a partir de $\sim 10^{5} \mathrm{GeV}$ e é o efeito dominante para os staus. Já para os múons só passa a ser dominante para energias acima de $10^{9} \mathrm{GeV}$. É importante notar que apesar da perda de energia por interação fotonuclear ser a dominante já para léptons pesados como o tau, ela é suprimida para os sléptons, quando comparada aos léptons, como veremos abaixo.

A contribuição na perda de energia dos (s)léptons devido a interações fotonucleares é dada por:

$$
b_{f n}(E)=\frac{N_{A}}{A} \int_{y_{\min }}^{y_{\max }} \int_{Q_{\min }^{2}}^{Q_{\max }^{2}} y \frac{d^{2} \sigma}{d y d Q^{2}} d Q^{2} d y
$$

com a transferência de energia (equação 2.25) entre $y_{\min }=\left[\left(M+m_{\pi}\right)^{2}-\right.$ $\left.M^{2}\right] /(2 M E)$ e $y_{\max }=1-m_{l} / E$, em que $E$ é a energia do (s)lépton; $M$ é a massa do próton e $m_{\pi}$ é a massa do píon; $Q_{\max }^{2}=2 M y E-\left[\left(M+m_{\pi}\right)^{2}-M^{2}\right]$ e $Q_{\min }^{2} \simeq \frac{m_{l}^{2} y^{2}}{(1-y)}$ correspondem ao intervalo de transferência de momento [7].

O fato de $Q_{\min }^{2}$ depender da massa do slépton faz com que o limite inferior de integração na equação anterior seja bem maior para os sléptons, suprimindo sua perda de energia em relação a dos léptons.

A seção de choque diferencial $\frac{d^{2} \sigma}{d y d Q^{2}}$, na equação 2.43 é dada por:

$$
\begin{aligned}
\frac{d^{2} \sigma}{d y d Q^{2}}= & \frac{\pi \alpha^{2}}{Q^{4}} \frac{F_{2}\left(x, Q^{2}\right)}{y}\left\{(2-y)^{2}-\right. \\
& \left.y^{2}\left(1+\frac{4 m_{l}^{2}}{Q^{2}}\right) \times \frac{\left(1+\frac{4 M^{2} x^{2}}{Q^{2}}\right)}{\left[1+R\left(x, Q^{2}\right)\right]}\right\},
\end{aligned}
$$

com $x$ sendo:

$$
x=\frac{Q^{2}}{2 M\left(E-E^{\prime}\right)} .
$$

Para a equação 2.44 foi adotado o limite de Callan-Gross, o que implica em $R\left(x, Q^{2}\right)=0$ para os valores de energia usados neste trabalho. $F_{2}\left(x, Q^{2}\right)$ inclui os fatores nucleares e é dado por [7]: 


$$
F_{2}\left(x, Q^{2}\right)=a(A, x) \frac{A}{2}(1+P(x)) F_{2}^{p},
$$

onde $a(A, x)$ é uma parametrização que leva em consideração o efeito de shadowing nuclear, dada por [17]:

$$
a(A, x)= \begin{cases}A^{-0,1}, & x<0,0014 \\ A^{0,069 \log _{10}(x)+0,097}, & 0,0014 \leq x \leq 0,04 \\ 1, & 0,04<x\end{cases}
$$

e $P(x)$ corresponde a um ajuste aos dados da colaboração Bologna-CernDubna-Munich-Saclay:

$$
P(x)=1-1,85 x+2,45 x^{2}-2,35 x^{3}+x^{4} .
$$

Para o cálculo de $F_{2}^{p}\left(x, Q^{2}\right)$ foi usada a parametrização ALLM97 [24, 25]:

$$
F_{2}^{p}=\frac{Q^{2}}{Q^{2}+m_{0}^{2}}\left[F_{2}^{P}\left(x, Q^{2}\right)+F_{2}^{R}\left(x, Q^{2}\right)\right]
$$

$\operatorname{com} F_{2}^{P}$ e $F_{2}^{R}$ dadas por:

$$
\begin{gathered}
F_{2}^{P}=c_{P}(t) x_{P}^{a_{P}(t)}(1-x)^{b_{P}(t)}, \\
F_{2}^{R}\left(x, Q^{2}\right)=c_{R}(t) x_{R}^{a_{R}(t)}(1-x)^{b_{R}(t)} .
\end{gathered}
$$

O parâmetro $t$ é dado por:

$$
t=\ln \left(\frac{\ln \left(Q^{2}+Q_{0}^{2}\right) / \Lambda^{2}}{\ln \left(Q_{0}^{2} / \Lambda^{2}\right)}\right)
$$

Nas equações 2.50 e 2.51 as funções $a, b$ e $c$ são escritas da seguinte maneira: 


$$
\begin{aligned}
& a_{R}(t)=a_{R 1}+a_{R 2} t^{a_{3 R}} \\
& c_{R}(t)=c_{R 1}+c_{R 2} t^{c_{R 3}} \\
& a_{P}(t)=a_{P 1}+\left(a_{P 1}-a_{P 2}\right)\left[\frac{1}{1+t^{a} P 3}-1\right] \\
& c_{P}(t)=c_{P 1}+\left(c_{P 1}-c_{P 2}\right)\left[\frac{1}{1+t^{c} P 3}-1\right] \\
& b_{P}(t)=b_{P 1}+b_{P 2}^{2} t^{b_{P 3}} \\
& b_{R}(t)=b_{R 1}+b_{R 2}^{2} t^{b_{R 3}}
\end{aligned}
$$

e $x_{P}$ e $x_{R}$ são dados por:

$$
\begin{aligned}
& x_{p}=\frac{Q^{2}+m_{P}^{2}}{Q^{2}+m_{P}^{2}+W^{2}-M^{2}}, \\
& x_{R}=\frac{Q^{2}+m_{R}^{2}}{Q^{2}+m_{R}^{2}+W^{2}-M^{2}},
\end{aligned}
$$

onde $m_{P}$ e $m_{R}$ são as massas efetivas de Pomeron e Reggeon, respectivamente. $W$ é a energia no centro de massa.

Os parâmetros usados na equação 2.53 são apresentados na Tabela 2.1 $[24,25]$.

Tabela 2.1: Valores dos parâmetros ALLM 97 retirados da referência [25].

\begin{tabular}{|cc||cc|}
\hline Parâmetro & ALLM97 & Parâmetro & ALLM97 \\
\hline$m_{0}^{2}\left(\mathrm{GeV}^{2}\right)$ & 0,31985 & $m_{P}^{2}\left(\mathrm{GeV}^{2}\right)$ & 49,457 \\
$m_{R}^{2}\left(G e V^{2}\right)$ & 0,15052 & $Q_{0}^{2}\left(G e V^{2}\right)$ & 0,52544 \\
$\Lambda^{2}\left(G e V^{2}\right)$ & 0,06527 & & \\
\hline$a_{P 1}$ & $-0,0808$ & $a_{R 1}$ & 0,58400 \\
$a_{P 2}$ & $-0,44812$ & $a_{R 2}$ & 0,37888 \\
$a_{P 3}$ & 1,1709 & $a_{R 3}$ & 2,6063 \\
$b_{P 1}$ & 0,36292 & $b_{R 1}$ & 0,01147 \\
$b_{P 2}$ & 1,8917 & $b_{R 2}$ & 3,7582 \\
$b_{P 3}$ & 1,8439 & $b_{R 3}$ & 0,49338 \\
$c_{P 1}$ & 0,28067 & $c_{R 1}$ & 0,80107 \\
$c_{P 2}$ & 0,22291 & $c_{R 2}$ & 0,97307 \\
$c_{P 3}$ & 2,1979 & $c_{R 3}$ & 3,4942 \\
\hline
\end{tabular}




\subsubsection{Alcance máximo de (s)léptons na Terra}

O alcance máximo (range) é a distância máxima de propagação de uma partícula, na qual sua energia é degradada a níveis inferiores ao limiar de detecção. Este parâmetro é crucial para determinar a taxa de eventos no detector, já que partículas produzidas além desta distância em relação a ele não podem atingi-lo. Além disso, também é usado para determinar a probabilidade de sobrevivência do neutrino, no percurso que vai do seu ponto de incidência na Terra até o ponto correspondente ao range da NLSP em relação ao detector.

Para determinar o alcance máximo dos (s)léptons ao percorrerem o interior da Terra, é necessário determinar sua energia e sua degradação, incluindo tanto a perda por radiação como a por ionização, bem como a densidade do meio. Partindo-se da equação 2.23 para a perda de energia, temos:

$$
\Delta E \sim \rho(x) \cdot[a(E)+b(E) \cdot E] \cdot \Delta x
$$

onde $\rho(x)$ é a densidade da Terra em função da posição (equação 2.20) e $\Delta E$ é a energia perdida pela partícula ao percorrer o trecho $\Delta x$. O alcance máximo é determinado numericamente: definimos um valor fixo para $\Delta x$ (uma pequena fração do raio da Terra) e calculamos a perda de energia para cada passo $\Delta x$ dado. Iteramos este processo enquanto a energia da partícula for maior do que o limiar de detecção.

Para determinar a energia com a qual a partícula atinge o detector, o processo iterativo é similar, contudo é pautado à posição da partícula estar contida no volume do detector e à partícula possuir energia residual maior do que o limiar de detecção.

Nas Figuras 2.14 a 2.16 apresentamos o comportamento do alcance máximo respectivamente para o múon, tau e o slépton, em função do cosseno do ângulo de incidência destas partículas (ver figura 2.8). Nos três casos foi considerado que as partículas incidem sobre a superfície da Terra e a distância máxima percorrida é definida pelo detector posicionado a cerca de $2 \mathrm{~km}$ de profundidade. É possível notar que os sléptons percorrem distâncias muito maiores dentro da Terra do que os múons ou taus, como esperado, visto que as partículas supersimétricas perdem muito menos energia por unidade de comprimento. 


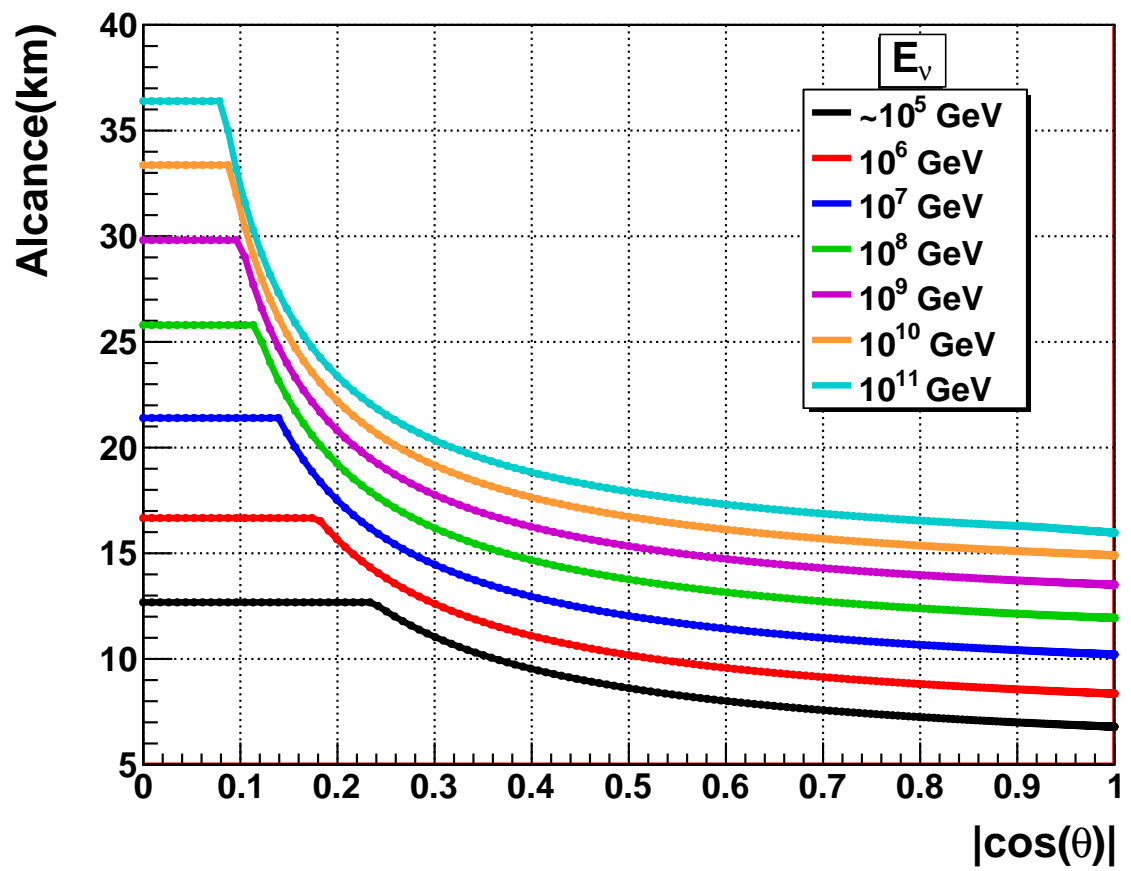

Figura 2.14: Alcance máximo para múons de diversas energias. Foi considerado que as partículas incidem sobre a superfície da Terra.

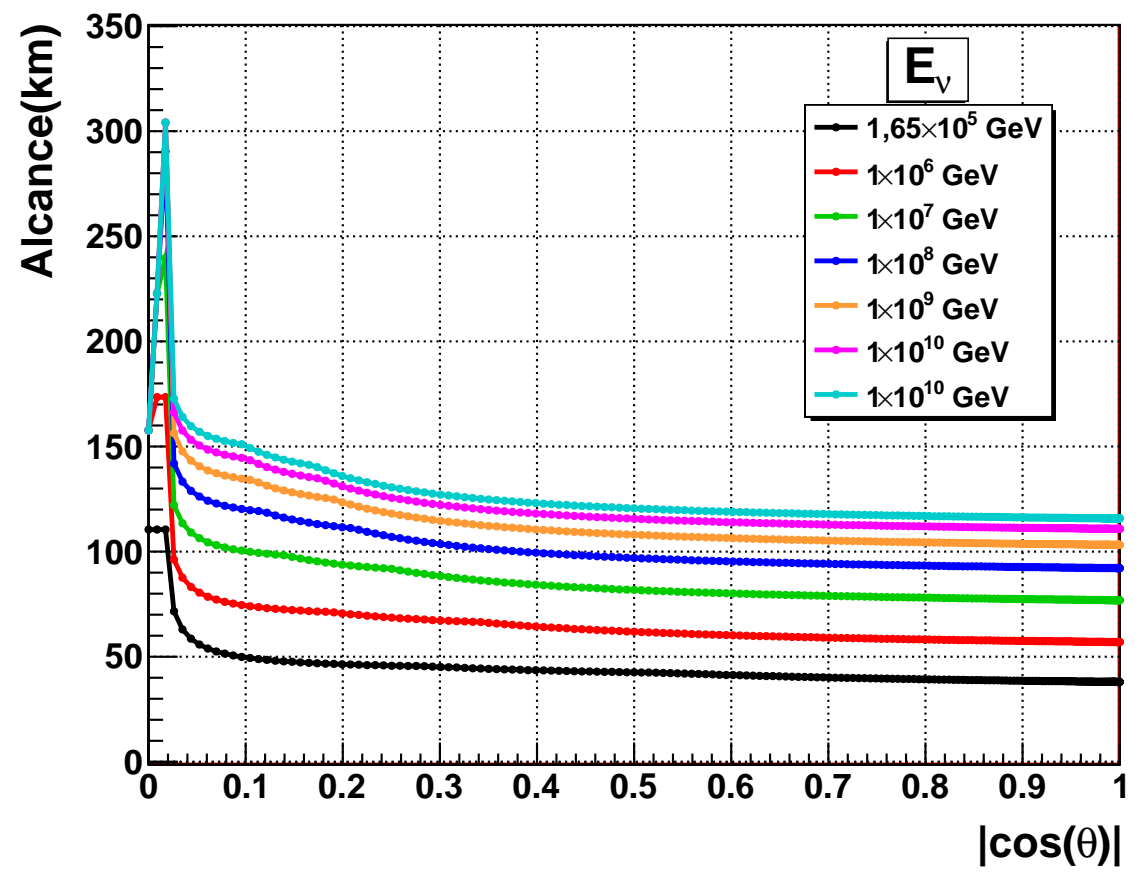

Figura 2.15: Alcance máximo para taus de diversas energias. Foi considerado que as partículas incidem sobre a superfície da Terra. 


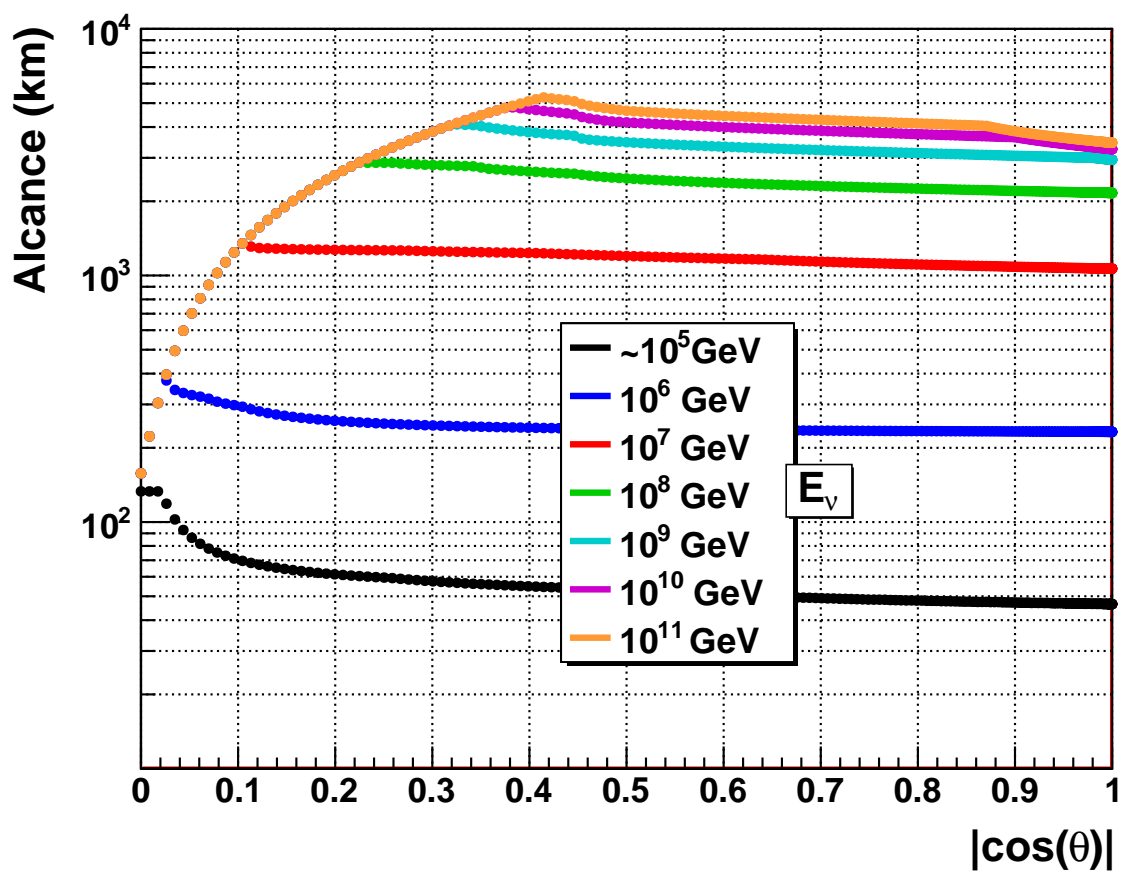

Figura 2.16: Alcance máximo para sléptons de diversas energias. Foi considerado que as partículas incidem sobre a superfície da Terra.

\subsection{Simulação de eventos no detector}

Nesta seção é descrito o procedimento adotado na simulação para se obter o fluxo de NLSPs no telescópio de neutrinos IceCube, assim como sua distribuição de energia. Os passos adotados foram os seguintes:

1) São gerados $N$ eventos correspondentes a neutrinos chegando à Terra para cada energia e ângulo considerados, tomando-se como referência a figura 2.8. O intervalo de energia considerado para os neutrinos que chegam à Terra compreende energias entre $\sim 1.6 \times 10^{5} \mathrm{GeV}$, que corresponde ao limiar de energia para produzir as NLSPs, e $10^{11} \mathrm{GeV}$, energia grande o suficiente para que o número de eventos correspondente seja desprezível. O intervalo de ângulos abrange o intervalo de 90 a 180 graus. Para ângulos menores que 90 graus, a profundidade do detector (cerca de $2 \mathrm{~km}$ ) é muito pequena em relação ao tamanho da Terra, o que reduz drasticamente a probabilidade do neutrino incidente produzir uma NLSP; 
2) Determina-se o ponto de interação para cada um dos $N$ eventos gerados, por meio de sorteio aleatório ${ }^{4}$ baseado na probabilidade do neutrino interagir produzindo um slépton, dada pela equação 2.18. Na figura 2.17 é mostrado um exemplo comparativo entre a distribuição obtida por meio dos sorteios para o ponto de produção de NLSPs e a curva correspondente à probabilidade considerada. Neste caso foi escolhida uma energia fixa para o neutrino incidente $\left(10^{9} \mathrm{GeV}\right)$ e um ângulo de incidência de 180 graus. Vale observar que para cada ângulo e energia tem-se uma distribuição diferente.

3) É verificado se o ponto de interação ocorre a uma distância para a qual a partícula produzida pode chegar ao detector, por meio do cálculo da alcance máximo (descrito na seção 2.5.4);

4) Se a partícula pode chegar ao detector, o evento é computado e a energia no detector é determinada como descrito também na seção 2.5.4. Determina-se assim, a fração de eventos $F\left(\theta, E_{\nu}\right)$ que chega ao detector entre os $N$ eventos gerados, considerando o ângulo e energia de incidência.

5) Um peso probabilístico para cada par ângulo/energia é dado pelo produto do fluxo de neutrinos dado pelo limite de WB (equação 2.3) e a probabilidade resultante da convolução entre a probabilidade de sobrevivência do neutrino (para produção de léptons) e a probabilidade de interagir (formação de NLSPs):

$$
P_{\text {peso }}=\left(\frac{d \phi_{\nu}}{d E}\right)_{\mathrm{WB}} \times P_{s_{\nu}}^{l}\left(E_{\nu}\right) \times P_{i_{\nu}}^{\tilde{\tau}}\left(E_{\nu}\right)
$$

onde $P_{s_{\nu}}^{l}\left(E_{\nu}\right)$ é dada pela equação 2.17 e $P_{i_{\nu}}^{\tilde{\tau}}\left(E_{\nu}\right)$ é dada pela equação 2.18. Este evento é convoluído com a fração de eventos $F\left(\theta, E_{\nu}\right)$.

\footnotetext{
${ }^{4} \mathrm{O}$ procedimento adotado para isto é detalhado no apêndice $\mathrm{C}$.
} 


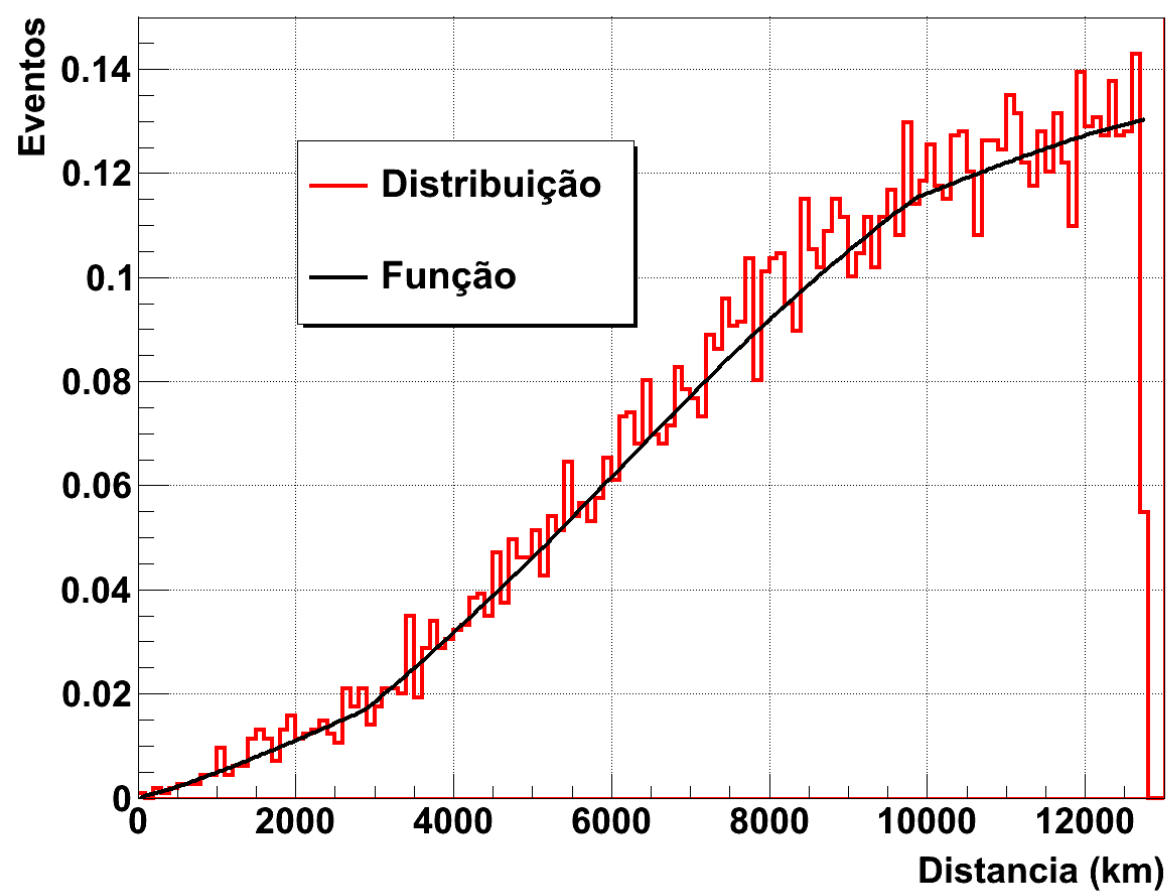

Figura 2.17: Em vermelho é apresentada a distribuição do ponto de produção dos staus no interior da Terra para um neutrino incidente com energia de $1 \times 10^{9}$ $\mathrm{GeV}$ e ângulo de 180 graus. O eixo $x$ representa o ponto de produção em relação ao ponto de incidência na superfície da Terra. A curva em preto corresponde à probabilidade de o neutrino interagir relativa à produção de NLSPs (equação 2.18).

\section{$2.7 \quad$ Resultados}

Seguindo os passos que descrevemos anteriormente, determinamos o espectro de energia das NLSPs que atingem o telescópio de neutrinos. A figura 2.18 mostra este espectro para os três valores adotados para a massa do squark produzido na interação neutrino-núcleon: 300, 600 e $900 \mathrm{GeV}$. Como já mencionado, esta escolha abrange um espectro de massas bem representativo para os cenários estudados neste trabalho. É importante observar que estas curvas são compatíveis com os resultados obtidos em [7]. 


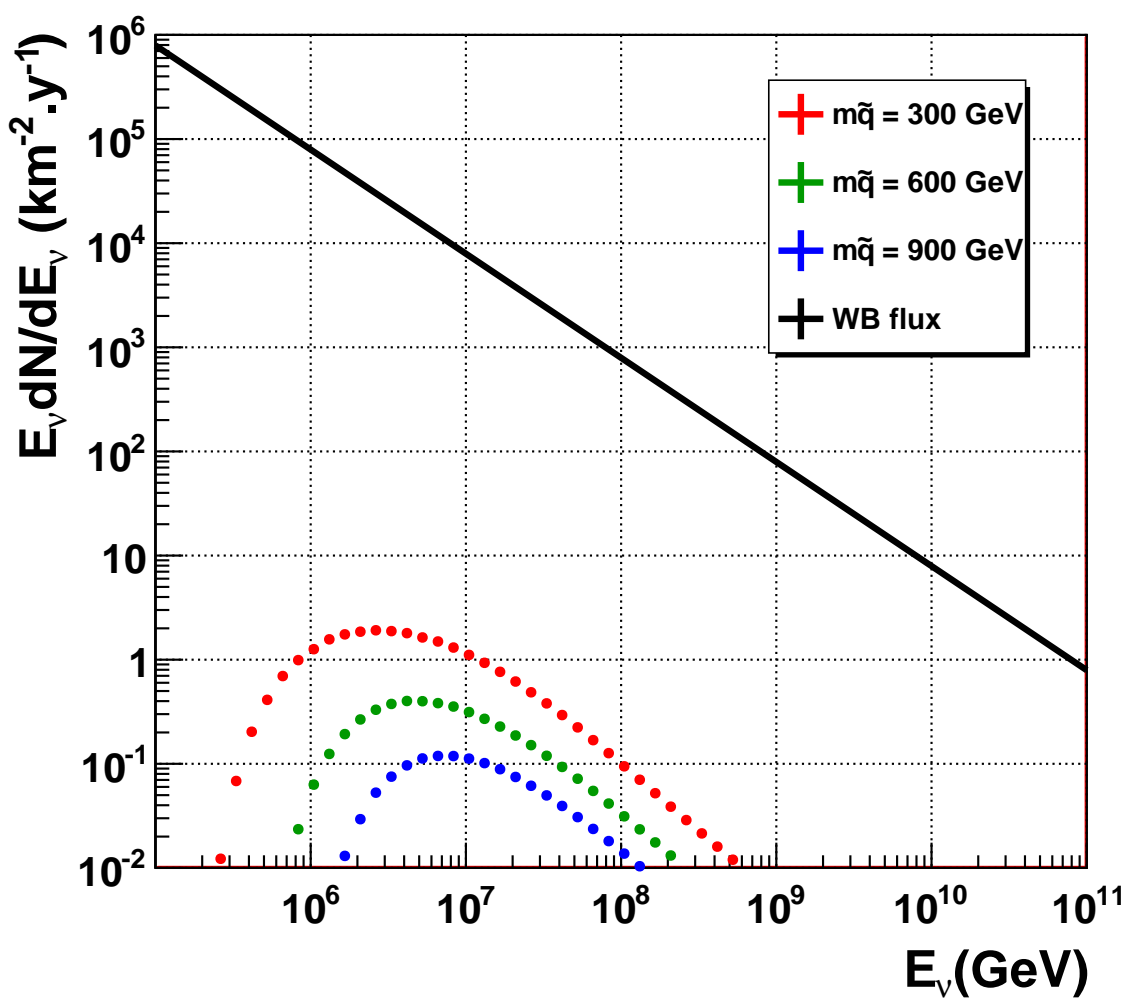

Figura 2.18: Eventos por $\mathrm{km}^{2}$ por ano num detector localizado a cerca de $2 \mathrm{~km}$ de profundidade. São apresentadas as curvas para três massas do squark produzido na interação neutrino-núcleon: 300, 600 e $900 \mathrm{GeV}$. É apresentado também, em preto, o limite de Waxman-Bahcall, que é adotado como o fluxo de neutrinos.

As taxas de NLSPs obtidas integrando as curvas apresentadas na figura 2.18, em função da energia de incidência do neutrino, são apresentados na tabela 2.2 e suas distribuições de energia de incidência no telescópio de neutrinos, na figura 2.19. Esta distribuição de energia é importante para a distinção entre as NLSPs e o fundo, constituído principalmente de dimúons provenientes de decaimento de partículas charmosas [7].

Tabela 2.2: Eventos de NLSPs por $\mathrm{km}^{2}$ por ano no telescópio de neutrinos posicionado a cerca de $2 \mathrm{~km}$ de profundidade na Terra.

\begin{tabular}{|c|c|c|c|}
\hline $\mathrm{M} \tilde{q}$ & $300 \mathrm{GeV}$ & $600 \mathrm{GeV}$ & $900 \mathrm{GeV}$ \\
\hline Eventos $\left(k m^{-2} y^{-1}\right)$ & 6 & 1 & 0,3 \\
\hline
\end{tabular}




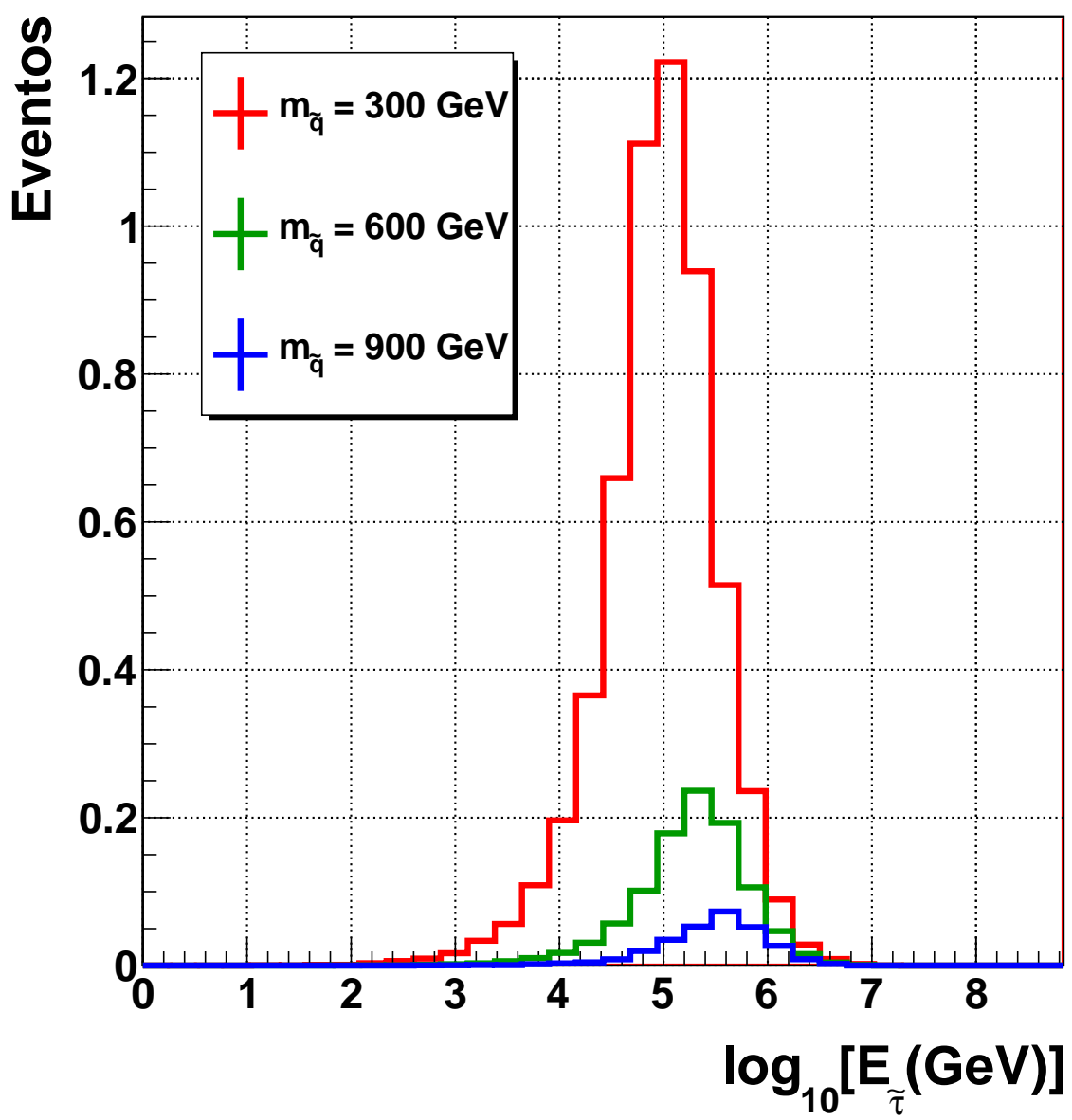

Figura 2.19: Energia de incidência das NLSPs no detector para três valores de massa do squark produzido na interação neutrino-núcleon: 300, 600 e $900 \mathrm{GeV}$. 


\section{Capítulo 3}

\section{Deteç̧ão Indireta de NLSPs: IceCube}

A partir deste capítulo tratamos da detecção indireta de NLSPs, considerando os cenários em que estas decaem. Com isto descrevemos a investigação original que realizamos, que é complementar aos resultados relativos à detecção direta de NLSPs. A NLSP decai em um tau mais um gravitino. Já o tau, por sua vez, decai quase que instantaneamente, tendo sempre o neutrino do tau entre os produtos deste decaimento. Analisamos aqui a viabilidade de detecção destes taus. Cabe lembrar que o gravitino é a LSP nos cenários de supersimetria considerados aqui e também candidato a matéria escura.

Como parte principal deste trabalho, simulamos o decaimento das NLSPs em duas etapas: i) primeiramente considerando que ele ocorre no interior da Terra e ii) considerando que ele ocorre na atmosfera, após estas partículas saírem do planeta, ou ainda no interior da Terra próximo à superfície de forma que o tau produzido possa emergir com energia suficiente para gerar um chuveiro atmosférico. Neste capítulo nos concentramos no primeiro caso, visando determinar a viabilidade de detecção por telescópios de neutrinos.

O tau produzido no decaimento da NLSP sofre o processo de regeneração: o neutrino do tau produzido em seu decaimento pode interagir por meio de corrente carregada e produzir um novo tau. Isto se repete, já que o tau decai novamente em um neutrino do tau. Ambas as partículas têm suas energias degradadas por este processo. Determinamos aqui a taxa de taus bem como sua distribuição de energia em um telescópio de neutrinos de volume de 1 
$\mathrm{km}^{3}$ a cerca de $2 \mathrm{~km}$ de profundidade como, por exemplo, o IceCube [8]. Um ponto a ser destacado é que o processo de regeneração do tau implica em um aumento de volume efetivo para produção de staus, pois aqueles que não atingiam o detector por perderem toda sua energia, no caso da detecção direta, agora podem decair antes disto, aumentando a chance de um tau chegar até ele.

Similarmente ao caso da detecção direta de NLSPs, a taxa de eventos depende do fluxo de neutrinos que incide sobre a Terra, das probabilidades do neutrino interagir e produzir partículas supersimétricas e da perda de energia das partículas produzidas durante sua propagação. Para a detecção indireta esta taxa também depende da probabilidade da NLSP decair e da regeneração do tau. O decaimento depende da escala de quebra de supersimetria $\sqrt{F}$ (vide equação 3.3) e exemplificamos nossos resultados com valores arbitrários de $\sqrt{F}$. Após simularmos a produção de NLSPs, determinamos sua propagação e o seu decaimento. Se a NLSP decair antes do detector, o decaimento do tau e sua eventual regeneração são analisados.

Estimamos também a taxa de taus e suas distribuições de energia em telescópios de neutrinos de volumes maiores [26], para detectores com raios de 2 e $4 \mathrm{~km}$ adicionais ao tamanho original, promovendo volumes de 21 e 65 $\mathrm{km}^{3}$.

Uma outra análise realizada consiste na determinação da taxa de taus e distribuições de energia em telescópios de neutrinos, considerando a produção direta de taus, ou seja, sem o intermédio da produção de NLSPs. Neste caso, o objetivo é verificar a consistência de nossa simulação da regeneração do tau. Para isto determinamos as taxas de taus para algumas assinaturas desta partícula que espera-se que sejam observadas no telescópio IceCube [27]. Comparamos nossos resultados com os obtidos em [28].

\subsection{Decaimento da NLSP}

O decaimento da $\operatorname{NLSP}(\tilde{\tau})$ se dá no seguinte canal:

$$
\tilde{\tau} \longrightarrow \tau+\text { gravitino }
$$

Para que estas partículas decaiam no interior da Terra antes de atingir o 
IceCube ou na atmosfera logo após se propagarem pelo planeta, a escala de quebra de supersimetria fica restrita ao intervalo $\sim 10^{5} \mathrm{GeV} \lesssim \sqrt{F} \lesssim 10^{7}$ $\mathrm{GeV}$. Este intervalo está relacionado à distância que o stau pode percorrer antes de decair (equação 3.2), que depende tanto de $\sqrt{F}$ como da energia da partícula. Descrevemos a dependência da probabilidade de decaimento da NLSP com sua energia e com $\sqrt{F}$ na próxima subseção e na sequência, a determinação da energia do tau produzido no decaimento da NLSP.

\subsubsection{Probabilidade de decaimento do stau}

A probabilidade de sobrevivência de uma partícula de massa $m$ e energia $E$ em relação ao seu decaimento é dada por [1]:

$$
P_{s d}=e^{-\frac{m \cdot x}{c \tau \cdot E}}
$$

Onde $x$ é a distância percorrida por ela. Aproximamos $E \sim k$, sendo $k$ o seu momento, pois $k \gg m$. Nos modelos que consideramos aqui, com quebra de supersimetria mediada por interação de gauge [5], o fator $c \tau$ é dado pela equação 2.1 .

Já a probabilidade de uma partícula decair é dada por:

$$
P_{\text {decair }}=1-P_{s d}
$$

Nas figuras 3.1 e 3.2 adiante, são apresentados gráficos que ilustram a dependência da probabilidade de sobrevivência do stau em função da energia do neutrino incidente e também de $\sqrt{F}$. Nota-se que esta probabilidade é altamente dependente destes dois parâmetros. 


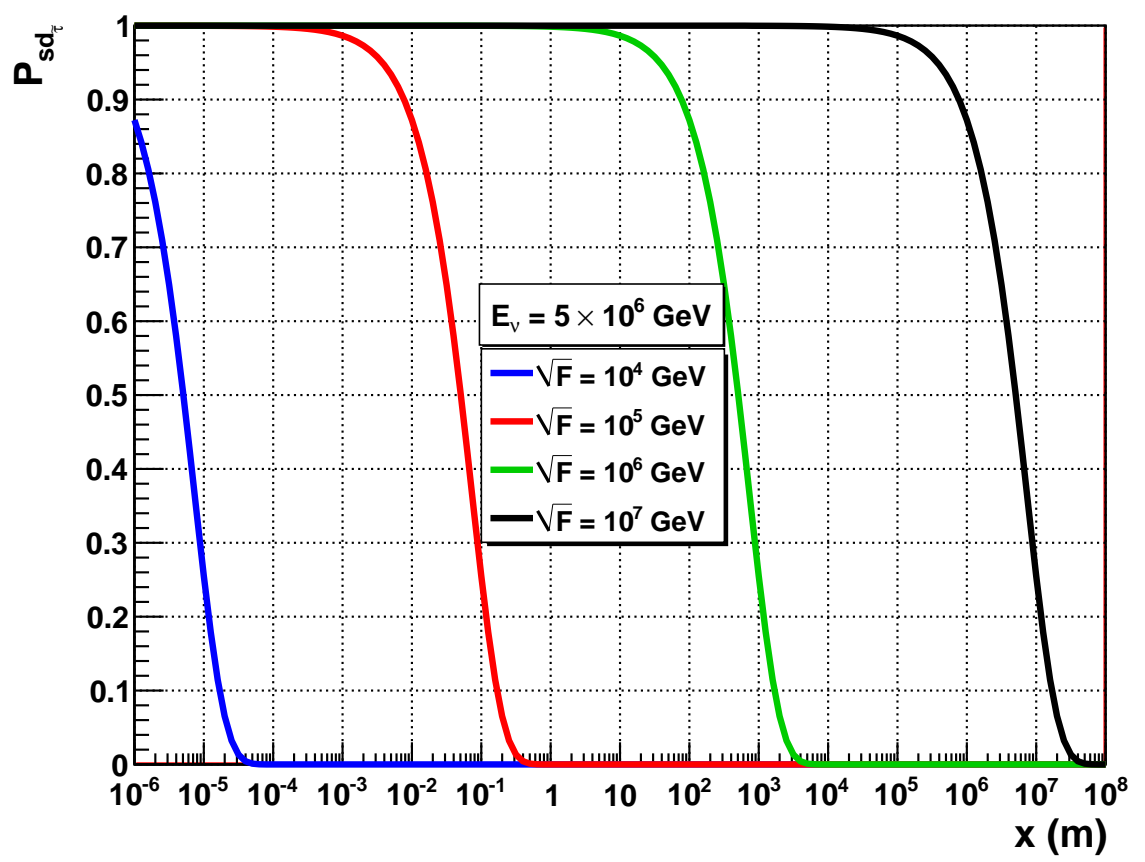

Figura 3.1: Probabilidade de sobrevivência do stau em função da distância percorrida para quatro valores de $\sqrt{F}$ e energia do neutrino de $5 \times 10^{6} \mathrm{GeV}$.

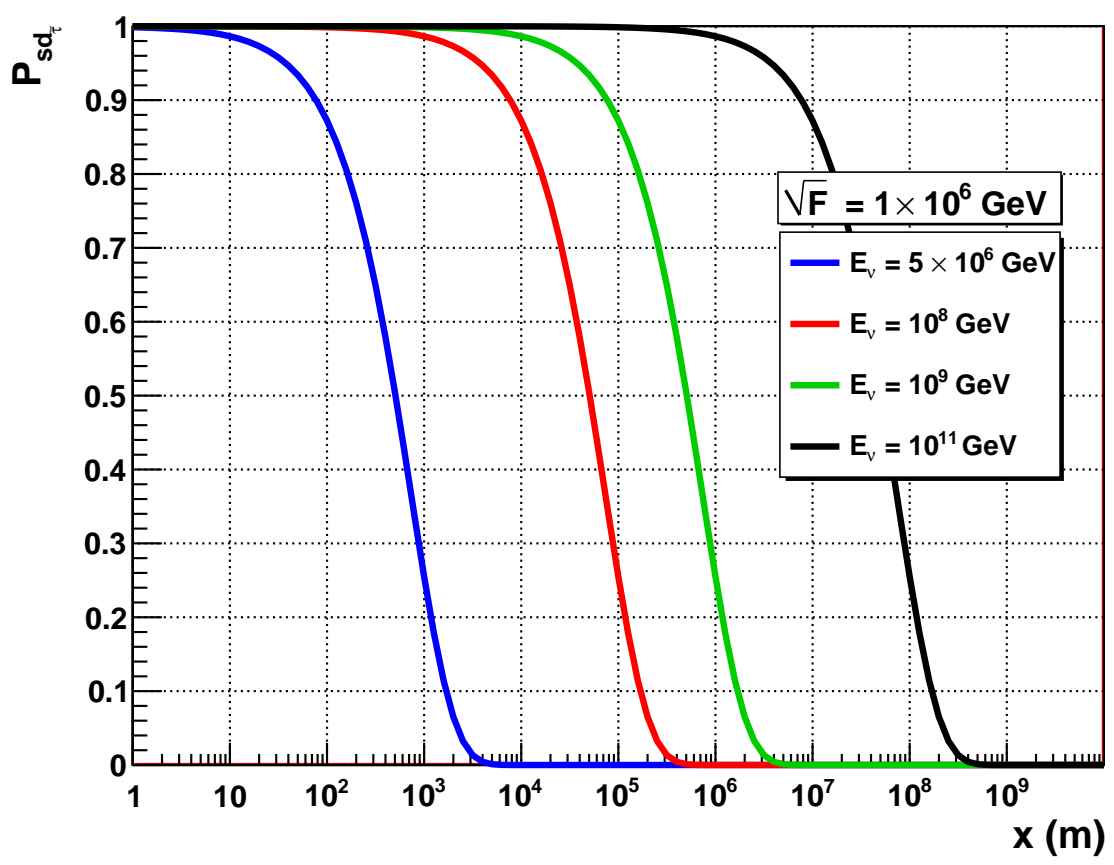

Figura 3.2: Probabilidade de sobrevivência do stau em função da distância percorrida para quatro valores de energia do neutrino $\left(E_{\nu}\right)$ e $\sqrt{F}=1 \times 10^{6} \mathrm{GeV}$. 
É possível notar na figura 3.1, na qual a energia do neutrino é mais próxima ao limiar de energia para a produção de staus, que as partículas têm uma chance maior de decair fora da Terra para um valor de $\sqrt{F}$ acima de $10^{7} \mathrm{GeV}$, lembrando que o raio da Terra é de cerca de $6,5 \times 10^{6} \mathrm{~m}$. Nota-se também que para cada fator $10 \mathrm{em} \sqrt{F}$ temos um fator de cerca de $10^{4}$ para a distância percorrida pelas partículas.

Já no caso da figura 3.2 em que $\sqrt{F}$ é fixo em $10^{6} \mathrm{GeV}$, observa-se que apenas para energias do neutrino acima de $10^{9} \mathrm{GeV}$ a probabilidade da partícula decair fora da Terra é alta. A dependência da probabilidade de sobrevivência do stau é menor em relação à energia do que em relação a $\sqrt{F}$, pois para cada fator 10 na energia temos um fator aproximadamente 10 de deslocamento da curva em relação à variação na distância.

\subsubsection{Cinemática do decaimento do stau}

Aqui descrevemos sucintamente a cinemática do decaimento em dois corpos para o stau e a determinação da energia inicial do tau ${ }^{1}$, que é produzido neste decaimento (equação 3.1). Os parâmetros conhecidos inicialmente são a massa do tau e a energia do stau, que é $1 / 6$ da energia do neutrino que incide sobre a Terra [6]. Nos cenários de supersimetria que adotamos a massa do gravitino é desprezível em relação à massa do tau. Por fim, o ângulo $\theta_{C M}$ (no centro de momentos - CM) entre as direções do stau e do tau, é determinado a partir de uma distribuição isotrópica [29].

A energia do tau no referencial do laboratório (Lab) é, então, dada por:

$$
E_{\tau_{L a b}}=\gamma_{C M}\left[E_{\tau_{C M}}+v_{C M}\left|\mathbf{p}_{\tau_{\mathrm{CM}}}\right| \cos \left(\theta_{C M}\right)\right]
$$

com o fator de Lorentz $\gamma_{C M}=E_{\tilde{\tau}_{L a b}} / m_{\tilde{\tau}}$. A energia, velocidade e momento do tau no CM são respectivamente:

$$
\begin{gathered}
E_{\tau_{C M}}=\frac{m_{\tilde{\tau}}^{2}+m_{\tau}^{2}}{2 m_{\tilde{\tau}}}, \\
v_{C M}=\sqrt{1-\frac{1}{\gamma_{C M}^{2}}} \mathrm{e}
\end{gathered}
$$

\footnotetext{
${ }^{1}$ A dedução é detalhada no apêndice D.2
} 


$$
\left|\mathbf{p}_{\tau_{\mathrm{CM}}}\right|=\frac{1}{2 m_{\tilde{\tau}}}\left(m_{\tilde{\tau}}^{2}-m_{\tau}^{2}\right)
$$

Em uma boa aproximação, a trajetória do tau produzido tem a mesma direção que a NLSP, ou seja, o ângulo $\left(\theta_{L a b}\right)$ entre a direção do stau e a do tau no referencial do laboratório é desprezível (vide apêndice D.3).

\subsection{Regeneração do tau}

Apesar de terem vida média curta, $(290.6 \pm 1.0) \times 10^{-15} \mathrm{~s}[1]$, taus com energias $\gtrsim 10^{6} \mathrm{GeV}$ podem percorrer uma distância considerável antes de decair (ver figura 3.3):

$$
\Delta x_{\tau}(k m)=\frac{E_{\tau}}{m_{\tau}} c \tau
$$

com $c \tau=8,711 \times 10^{-8} \mathrm{~km}$ [1]. Sendo assim, a distribuição de probabilidade de decaimento do tau (equação 3.3) determina sua propagação antes de seu decaimento.

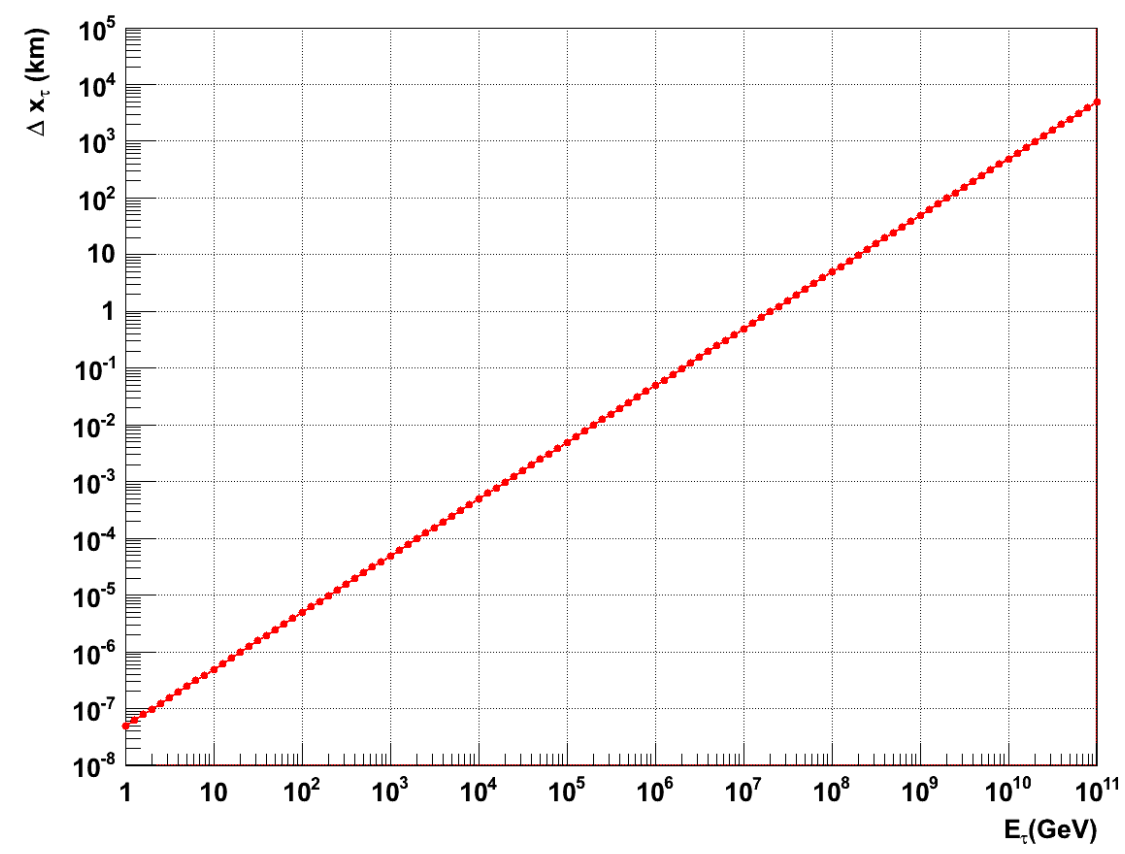

Figura 3.3: Distância média percorrida pelo tau, antes de decair, em função de sua energia. 
Apesar de existirem muitos canais de decaimento do tau, em todos eles sempre há a produção de um neutrino do tau $\left(\nu_{\tau}\right)[1]$ :

$$
\tau \longrightarrow \nu_{\tau}+X
$$

O neutrino do tau, por sua vez, pode interagir com um núcleon no interior da Terra e produzir um novo tau por meio de interação de corrente carregada $(2.2)$ :

$$
\nu_{\tau} N \stackrel{c c}{\longrightarrow} \tau+X
$$

onde a probabilidade desta interação com um núcleon no interior da Terra $\left(P_{i_{\nu}}^{\tau}\right)$ é dada pela equação 2.18 com $\sigma_{\nu}$ sendo a seção de choque inclusiva de corrente carregada para produção de léptons $\left(\sigma_{\nu}^{l}\right)$, dada pela equação 2.9 . Vale lembrar que para energias relevantes em nossa análise, as seções de choque para múons e taus são equivalentes ${ }^{2}$ [15].

A energia do neutrino do tau pode ser determinada através da distribuição $\frac{d n}{d z}$, descrita em mais detalhes nas referências [30,31], que relaciona a energia dos produtos de decaimento do tau com sua energia:

$$
\frac{d n}{d z}=\sum_{i} B_{i}\left(g_{0}^{i}-g_{1}^{i}\right)
$$

onde $z=E_{\nu_{\tau}} / E_{\tau}$ é a razão entre as energias do neutrino do tau $\left(E_{\nu_{\tau}}\right)$ e a do tau $\left(E_{\tau}\right)$ e $B_{i}$ é a fração do i-ésimo canal de decaimento do tau. Na tabela 3.1 são apresentados os coeficientes $g_{0}^{i}, g_{1}^{i}$ e as frações dos canais de decaimento do tau, $B_{i}$, da distribuição $\frac{d n}{d z}$. Esta distribuição é normalizada da seguinte maneira:

$$
\int \frac{d n}{d z} d z=\sum_{i} B_{i}=1
$$

Desta forma, a energia do neutrino do tau é dada por $E_{\nu_{\tau}}=z E_{\tau}$, onde $z$ é obtido aleatoriamente ${ }^{3}$ a partir da distribuição $\frac{d n}{d z}$. Esta é apresentada na figura 3.4, que reproduz bem a determinada em [30].

\footnotetext{
${ }^{2}$ Da mesma forma, a energia carregada pelo múon e pelo tau na interação neutrinonúcleon são equivalentes.

${ }^{3} \mathrm{O}$ procedimento adotado para sortear os valores de $\mathrm{z}$ é descrito no apêndice $\mathrm{C}$
} 
Tabela 3.1: Funções $g_{0}$ e $g_{1}$ para a distribuição de energia do neutrino do tau proveniente do decaimento do tau, em função de $z=E_{\nu_{\tau}} / E_{\tau}$. X indica todos os hádrons excetuando-se os píons $(\pi), \rho$ e $a_{1} \cdot r_{i}=m_{i}^{2} / m_{\tau}^{2}$. A função $\theta$ é a função degrau. Dados obtidos de [30].

\begin{tabular}{lccc}
\hline Processo & $B_{\tau}$ & $g_{0}$ & $g_{1}$ \\
\hline \hline$\tau \rightarrow \nu_{\tau} \mu \nu_{\mu}$ & 0,18 & $\frac{5}{3}-3 z^{2}+\frac{4}{3} z^{3}$ & $\frac{1}{3}-3 z^{2}+\frac{8}{3} z^{3}$ \\
$\tau \rightarrow \nu_{\tau} e \nu_{e}$ & 0,18 & $\frac{5}{3}-3 z^{2}+\frac{4}{3} z^{3}$ & $\frac{1}{3}-3 z^{2}+\frac{8}{3} z^{3}$ \\
$\tau \rightarrow \nu_{\tau} \pi$ & 0,12 & $\frac{1}{1-r_{\pi}} \Theta\left(1-r_{\pi}-z\right)$ & $-\frac{2 z-1+r_{\pi}}{\left(1-r_{\pi}\right)^{2}} \Theta\left(1-r_{\pi}-z\right)$ \\
$\tau \rightarrow \nu_{\tau} \rho$ & 0,26 & $\frac{1}{1-r_{\rho}} \Theta\left(1-r_{\rho}-z\right)$ & $-\left(\frac{2 z-1+r_{\rho}}{\left(1-r_{\rho}\right)}\right)\left(\frac{1-2 r_{\rho}}{1+2 r_{\rho}}\right) \Theta\left(1-r_{\rho}-z\right)$ \\
$\tau \rightarrow \nu_{\tau} a_{1}$ & 0,13 & $\frac{1}{1-r_{a_{1}}} \Theta\left(1-r_{a_{1}}-z\right)$ & $-\left(\frac{2 z-1+r_{a_{1}}}{\left(1-r_{a_{1}}\right)}\right)\left(\frac{1-2 r_{a_{1}}}{1+2 r_{a_{1}}}\right) \Theta\left(1-r_{a_{1}}-z\right)$ \\
$\tau \rightarrow \nu_{\tau} X$ & 0,13 & $\frac{1}{0,3} \Theta(0,3-z)$ & 0 \\
\hline
\end{tabular}

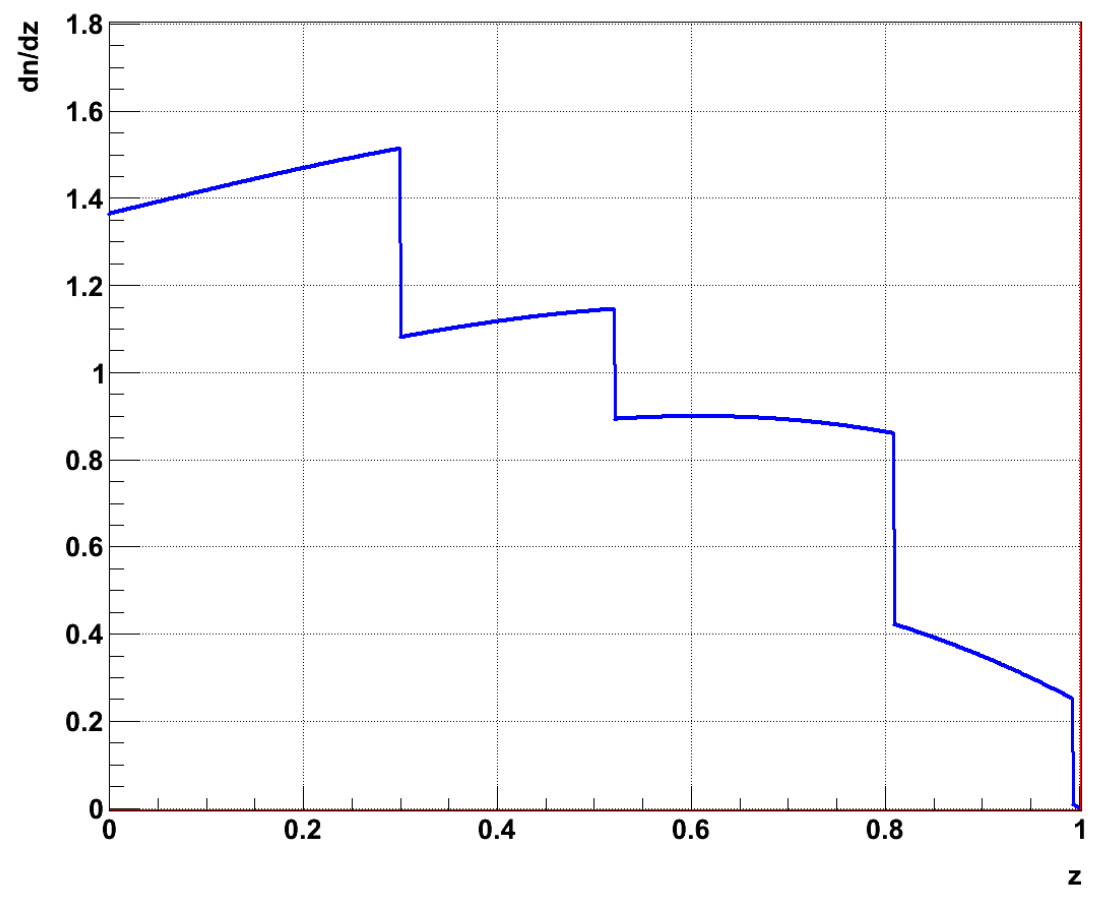

Figura 3.4: Distribuição $\frac{d n}{d z}$ em função de $z$, que relaciona as energias dos produtos de decaimento do tau com a energia do mesmo. 


\subsection{Simulação de eventos}

Para simular a detecção indireta de NLSPs, por meio dos taus, a geometria do detector é muito relevante, pois a distância percorrida por estas partículas antes de decair varia muito com a energia. Para energias até cerca de $10^{6} \mathrm{GeV}$, a distância média percorrida pelo tau é muito menor do que as dimensões do detector. Desta forma a taxa de eventos deve ser determinada em relação ao volume do detector. Deve-se também levar em conta a geometria, dado que as probabilidades de sobrevivência/interação do neutrino são altamente dependentes das trajetórias percorridas no interior da Terra. Sendo assim, consideramos uma geometria aproximada do IceCube, aproximando-o como um cilindro de $0,564 \mathrm{~km}$ de raio e $1 \mathrm{~km}$ de altura, cujo volume resultante é aproximadamente $1 \mathrm{~km}^{3}$. Já para a detecção direta de NLSPs a geometria do detector não foi levada em consideração, onde tomamos apenas um ponto de referência e determinamos a taxa por unidade de área no mesmo. Neste caso esta taxa de eventos por unidade de área é, numa boa aproximação, constante em todo o volume do detector, pois a distância percorrida pelas NLSPs é muito grande quando comparada às dimensões do IceCube.

Para obter as trajetórias das partículas sorteamos, através de uma distribuição uniforme, dois pontos na superfície do detector $\mathrm{P}_{1}$ e $\mathrm{P}_{2}$ exemplificados na figura 3.5. Este par de pontos determina uma reta cujo ponto de intersecção com a superfície da Terra nos fornece o ponto de entrada do neutrino incidente. A referência passa a ser o centro da Terra com cada ponto da trajetória descrito em função do raio interno da Terra. Desta forma o perfil de densidade da Terra é determinado diretamente pela equação 2.20. Como no caso da detecção direta de NLSPs, foram excluídas as trajetórias "de cima para baixo", ou seja, as que formam um ângulo acima da horizontal, pois de forma geral, representam os eventos gerados por neutrinos atmosféricos, que não produzem NLSPs. Vale observar que excetuando-se esta restrição de trajetórias possíveis, todas as outras são levadas em consideração, como por exemplo, quando o ponto de incidência no detector está na superfície inferior do cilindro. Os detalhes de como as trajetórias são calculadas encontram-se no apêndice E. 


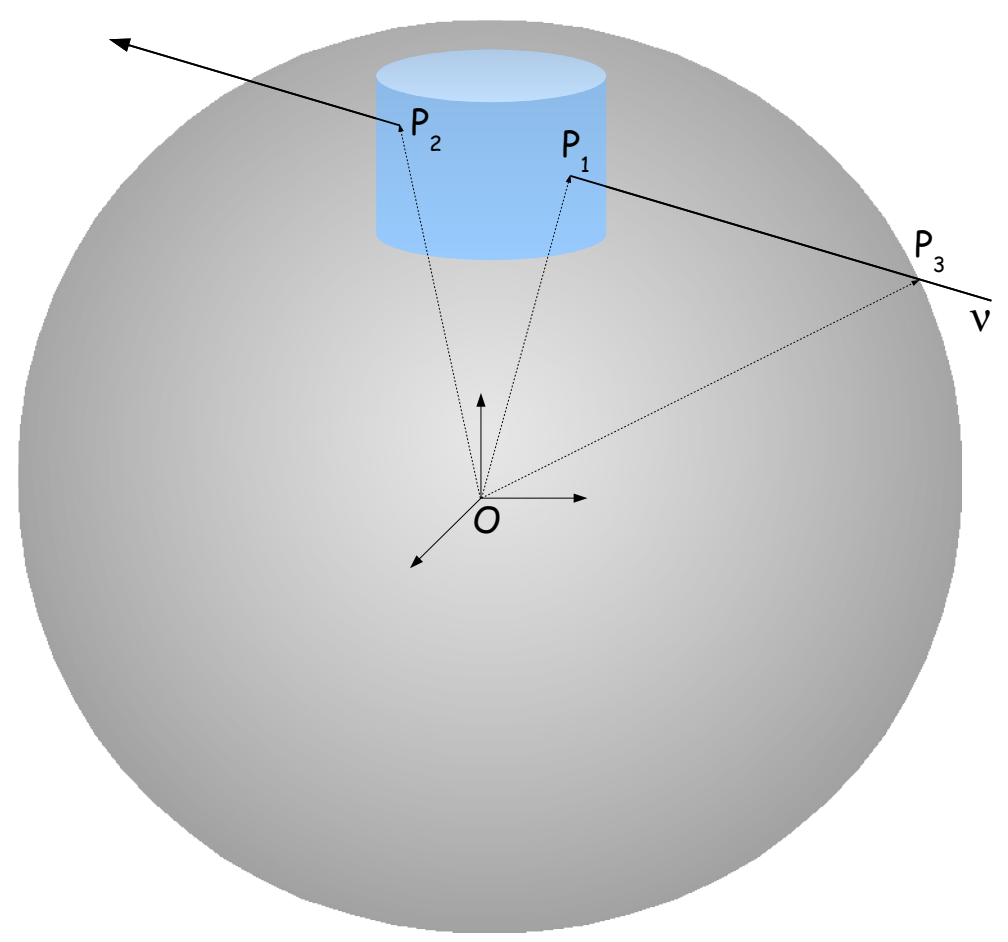

Figura 3.5: Esquema da geometria utilizada para geração das trajetórias que passam por um telescópio de neutrinos a cerca de $2 \mathrm{~km}$ de profundidade, cuja forma foi aproximada por um cilindro.

A seguir são descritos os passos adotados na simulação Monte Carlo para se determinar a taxa de eventos e a distribuição de energia dos taus quando atingem o telescópio de neutrinos IceCube. Toda a sequência apresentada é feita para valores arbitrários de $\sqrt{F}$ :

1) Geram-se $N$ trajetórias ${ }^{4}$, tais como descrito acima, correspondentes a neutrinos que chegam à Terra, para cada energia considerada. $\mathrm{O}$ intervalo de energia adotado para os neutrinos incidentes compreende energias entre $\sim 1.6 \times 10^{5} \mathrm{GeV}$, que corresponde à energia mínima necessária para produzir as NLSPs, e $10^{11} \mathrm{GeV}$, energia grande o suficiente para que o número de eventos seja desprezível;

\footnotetext{
${ }^{4}$ Lembrando que a normalização do fluxo de neutrinos é dada pelo limite de Waxman e Bahcall [11, 12].
} 
2) Dada a distribuição de produção de NLSPs (equação 2.18), a partir das interações de neutrinos com núcleons da Terra, o ponto de decaimento é sorteado a partir da distribuição de probabilidade de decaimento (equação 3.3). A energia do tau produzido antes do detector é determinada por meio da equação 3.4, lembrando que a perda de energia da NLSP até o ponto de decaimento é levada em consideração. A partir de então entra-se no ciclo de regenerações do tau. Nos casos em que a NLSP decai após o detector o evento é contado para normalização;

3) Para cada ciclo $j$ de regeneração do tau, o ponto de seu decaimento é determinado por meio de sorteio aleatório baseado na distribuição de probabilidade definida pelas equações 3.2 e 3.3. Se o decaimento ocorrer antes do detector, determinamos a perda de energia correspondente ao trecho percorrido e em seguida, a energia do novo neutrino do tau por meio da distribuição 3.11. Verificamos, então, baseado na distribuição de probabilidade dada pela equação 2.18, se este neutrino interage com um núcleon dentro da Terra. Continuamos este processo até que o decaimento do tau ocorra no (ou após o) volume do detector;

4) Aqui consideramos um peso probabilístico dado pela convolução da probabilidade de sobrevivência do neutrino (para produção de léptons) com a probabilidade de interagir (formação de NLSPs) para cada trajetória. O fluxo de neutrinos, dado pelo limite de WB (equação 2.3) é considerado apenas no final da contagem de eventos;

5) Um evento correspondente a um tau atingindo o detector é contabilizado quando esta partícula é produzida no volume compreendido pelo detector ou o atinge antes de decair. Ao final de cada ciclo de $N$ trajetórias geradas, temos a fração $F\left(E_{\nu}\right)$ de eventos válidos no volume do detector. Esta fração de eventos é independente do fluxo de neutrinos;

6) O número de eventos por ano no volume do detector, para cada energia, normalizado pelo limite de Waxman e Bahcall é dado pelo fluxo de partículas que atingem a superfície do detector multiplicado pela fração de eventos $F\left(E_{\nu}\right)$ : 


$$
\phi\left(E_{\nu}\right)=F\left(E_{\nu}\right) \times\left(\frac{d \phi_{\nu}}{d E_{\nu}}\right)_{\mathrm{WB}} \times A_{\text {detector }}
$$

onde $A_{\text {detector }}$ é a soma das áreas da superfície lateral e da superfície inferior do cilindro da figura 3.5. O número total de eventos por ano no volume do detector é dado pela integral na energia da equação 3.13.

\subsubsection{Múons provenientes do decaimento do tau}

Múons são produzidos em 18\% dos decaimentos do tau [1]. Desta forma, devemos contabilizar a contribuição dos múons como sinais de decaimento de staus. Para isto, simulamos a produção e propagação de múons em $18 \%$ dos decaimentos do tau, de forma equivalente à simulação do tau. Ressaltamos que o múon não sofre o processo de regeneração, dado que sua vida média é bem maior do que a do tau, e em média ele perde a maior parte de sua energia antes de decair. Por outro lado, em média percorre distâncias maiores que o tau. Sendo assim, também determinamos a taxa de múons no volume do telescópio de neutrinos bem como a distribuição de energia ao atingi-lo. Vale observar que a energia de cada múon produzido no decaimento do tau também é obtida por meio da distribuição $\frac{d n}{d z}$ (tabela 3.1).

\subsection{Resultados}

São apresentados aqui resultados de diferentes tipos de simulações para as quais o telescópio IceCube é tomado como referência. O primeiro caso refere-se à determinação da taxa de taus provenientes do decaimento das NLSPs para um telescópio de neutrinos de $1 \mathrm{~km}^{3}$ de volume (seção 3.4.1). Em seguida, na seção 3.4.2, são apresentados resultados similares para telescópios de neutrinos de volumes maiores do que $1 \mathrm{~km}^{3}$. Por fim, na seção 3.4 .3 , temos os resultados para os casos em que consideramos a produção direta de taus. 


\subsubsection{Taus originados em decaimentos de NLSPs}

Aqui são apresentados os resultados obtidos para a taxa de taus provenientes do decaimento das NLSPs em um telescópio de neutrinos de $1 \mathrm{~km}^{3}$ de volume, onde tomamos como padrão a geometria e localização do IceCube [37]. Incluímos também a taxa e distribuição de energia de múons provenientes do decaimento do tau. Estes resultados são dados em função de valores arbitrários de $\sqrt{F}$, sempre para os três valores de massa do squark considerados: 300, 600 e $900 \mathrm{GeV}$. O espectro adotado para $\sqrt{F}$ abrange valores entre $6 \times 10^{4}$ e $5 \times 10^{7} \mathrm{GeV}$ : o valor mínimo é tal que a probabilidade de decaimento das NLSPs na Terra é máxima, enquanto o valor máximo garante que não decaiam dentro da Terra. Desta forma determinamos a viabilidade de detecção indireta de NLSPs bem como de testar o intervalo para a escala de quebra de supersimetria.

Inicialmente apresentamos os espectros de energia obtidos para taus, múons e staus no volume do telescópio de neutrinos de $1 \mathrm{~km}^{3}$ para $\sqrt{F}$ $=1 \times 10^{5} \mathrm{GeV}$, para o qual a taxa de taus é máxima. Na figura 3.6 temos três gráficos com cinco curvas em cada, que representam taxas de eventos por ano em função da energia do neutrino que incide sobre a Terra, para $m_{\tilde{q}}=300,600$ e $900 \mathrm{GeV}$, respectivamente. Também é mostrado o limite de Waxman e Bahcall, que tomamos como fluxo de neutrinos incidentes na Terra. A curva com mais eventos (quadrados azuis) representa o espectro de pares de NLSPs produzidos. Os círculos vermelhos representam os resultados da detecção direta (pares de NLSPs), para $\sqrt{F}=5 \times 10^{7} \mathrm{GeV}$, onde não há decaimentos de NLSPs. A diferença entre estas duas curvas é atribuída às partículas que perdem toda sua energia antes de chegarem ao detector. É interessante observar que os resultados obtidos para a detecção direta aqui, levando-se em conta a geometria do detector, são equivalentes aos obtidos no capítulo anterior e os de $[6,7]$, indicando a compatibilidade entre o nosso trabalho em função do volume com o tratamento dos dados em função da área. Os círculos magenta representam os taus que chegam ao telescópio de neutrinos, quando $\sqrt{F}=1 \times 10^{5} \mathrm{GeV}$. Os quadrados verdes representam os múons provenientes do decaimento do tau, para o mesmo valor de $\sqrt{F}$. Por fim, as estrelas pretas representam a soma dos dois últimos casos. Nota-se que para energias do neutrino incidente até cerca de $10^{7} \mathrm{GeV}$ o número de 
taus é significativamente menor do que o de múons. Isto fica mais evidente no caso de $m_{\tilde{q}}=300 \mathrm{GeV}$, pois a energia mínima para produzir NLSPs é menor. Isto é esperado dado que o tau percorre distâncias muito menores do que as dimensões do detector antes de decair, o que diminui as chances de um evento no volume do detector. A partir desta energia, a relação passa a mudar, invertendo-se a energias maiores que $10^{8} \mathrm{GeV}$. Vemos também que a partir de cerca de $5 \times 10^{9}$ praticamente todos os staus produzidos geram taus que chegam ao detector.

A taxa total por ano no volume do detector para cada caso é obtida integrando a taxa de eventos em função da energia. É importante observar que, nos casos dos taus e múons, as curvas da figura 3.6 são determinadas para apenas um stau do par produzido, resultando em metade dos eventos que atingem o detector. O número total de eventos por ano, em $1 \mathrm{~km}^{3}$, é mostrado na tabela 3.2. No caso da detecção direta [6, 7], as duas NLSPs do par produzido se propagam juntas, com um ângulo de separação entre elas muito pequeno. Desta forma elas chegam juntas ao detector, produzindo um sinal muito particular no mesmo, o que permite distinguí-lo do fundo. No nosso caso, as NLSPs do par produzido podem decair em momentos diferentes e para avaliarmos estas coincidências seria necessário seguir duas cadeias de regenerações do tau paralelas. A quantidade de eventos que satisfazem esta condição é muito pequena ${ }^{5}$. Assim, o fundo para os eventos simulados aqui não são compostos de sinais coincidentes. Como será discutido no capítulo 5, o principal fundo para os múons é composto de eventos provenientes de neutrinos atmosféricos. Já os taus podem provocar assinaturas muito particulares no telescópio de neutrinos, praticamente livres de fundo, porém limitadas pelo tamanho do detector.

\footnotetext{
${ }^{5}$ Para os eventos na atmosfera as coincidências são levadas em consideração (próximo capítulo), pois a janela espacial para que elas ocorram é de no mínimo $100 \mathrm{~km}$.
} 

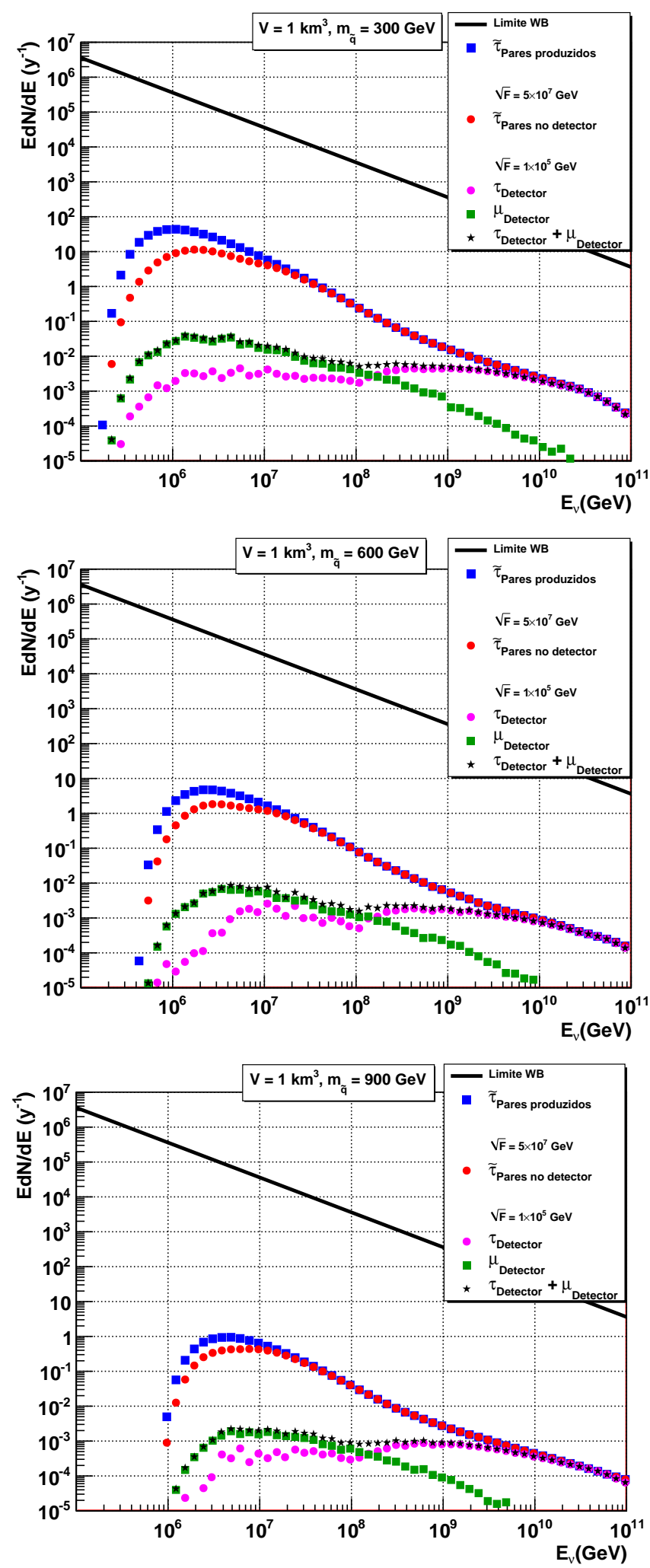

Figura 3.6: Fluxos por ano no volume de um telescópio de neutrinos de $1 \mathrm{~km}^{3}$. Também é apresentado o fluxo do total de NLSPs produzidas e o limite de Waxman e Bahcall. 
Tabela 3.2: Taxas de taus, múons e staus por ano em um telescópio de neutrinos de $1 \mathrm{~km}^{3}$ situado a cerca de $2 \mathrm{~km}$ de profundidade, para três valores de massa do squark produzido na interação neutrino-núcleon. No caso dos staus a taxa apresentada é o número de pares. $\sqrt{F}=1 \times 10^{5} \mathrm{GeV}$ para taus e múons e $5 \times 10^{7}$ $\mathrm{GeV}$ para staus.

\begin{tabular}{|c|c|c|c|c|}
\hline $\mathrm{m}_{\tilde{q}}(\mathrm{GeV})$ & $\tilde{\tau}\left(\mathrm{y}^{-1}\right)$ & $\tau\left(\mathrm{y}^{-1}\right)$ & $\mu\left(\mathrm{y}^{-1}\right)$ & $\mu+\tau\left(\mathrm{y}^{-1}\right)$ \\
\hline 300 & 27,7 & 0,07 & 0,21 & 0,28 \\
600 & 4,5 & 0,02 & 0,04 & 0,06 \\
900 & 1,1 & 0,01 & 0,01 & 0,02 \\
\hline
\end{tabular}

Na figura 3.7 são apresentadas as distribuições de energia dos taus e dos múons que atingem o detector. A normalização é baseada na tabela 3.2. No caso do tau temos uma distribuição dominada por eventos provenientes dos staus mais energéticos, com um máximo entre $10^{7}$ e $10^{8} \mathrm{GeV}$. Este efeito pode ser notado, em parte, na figura 3.6, onde há um máximo de eventos para energias de incidência por volta de $10^{9} \mathrm{GeV}$. Outro fator é o decaimento do tau para energias abaixo destes valores, onde a partícula percorre distâncias muito menores do que as dimensões do detector, diminuindo suas chances de atingi-lo. Ou seja, o número de eventos nestas condições é dominado pelos casos em que o tau é produzido dentro do volume do detector. Este efeito fica mais claro na seção 3.4.3, onde temos resultados para diferentes tipos de assinaturas do tau no detector. A distribuição de energia dos múons tem seu máximo a uma energia menor, por volta de $10^{4} \mathrm{GeV}$. Isto ocorre porque de forma geral o múon percorre distâncias maiores do que o tau e em contra partida perde mais energia por unidade de comprimento (seção 2.5). Para ambos os casos as distribuições obtidas para as massas mais pesadas de squark possuem uma forma similar, tendo como principal diferença apenas a quantidade de eventos.

Um parâmetro também interessante é o número de regenerações do tau. Na figura 3.8 são apresentados os números médios de regenerações relativos aos taus que chegam ao detector, em função da energia do neutrino que incide sobre a Terra, para os 3 valores de massa do squark produzido na interação neutrino-núcleon. O comportamento das três curvas é muito parecido, ficando claro que este parâmetro não influencia no número de regenerações. Isto é esperado dado que a probabilidade de decaimento das NLSPs é in- 
dependente da massa do squark. Nota-se que há um valor máximo para o número de regenerações quando a energia do neutrino incidente é cerca de $10^{8} \mathrm{GeV}$. Para energias mais altas há menos regenerações, pois as NLSPs e os taus conseguem percorrer maiores distâncias antes de decair (figuras $3.2 \mathrm{e}$ 3.3). No caso das energias mais baixas, há menos regenerações porque a probabilidade de interação do neutrino do tau é menor (figura 2.5). O máximo ocorre de tal forma a representar uma transição entre o regime de baixa probabilidade do neutrino interagir para o de maior distância percorrida pelo tau antes de decair, com energia suficientemente grande para ocorrerem mais regenerações.

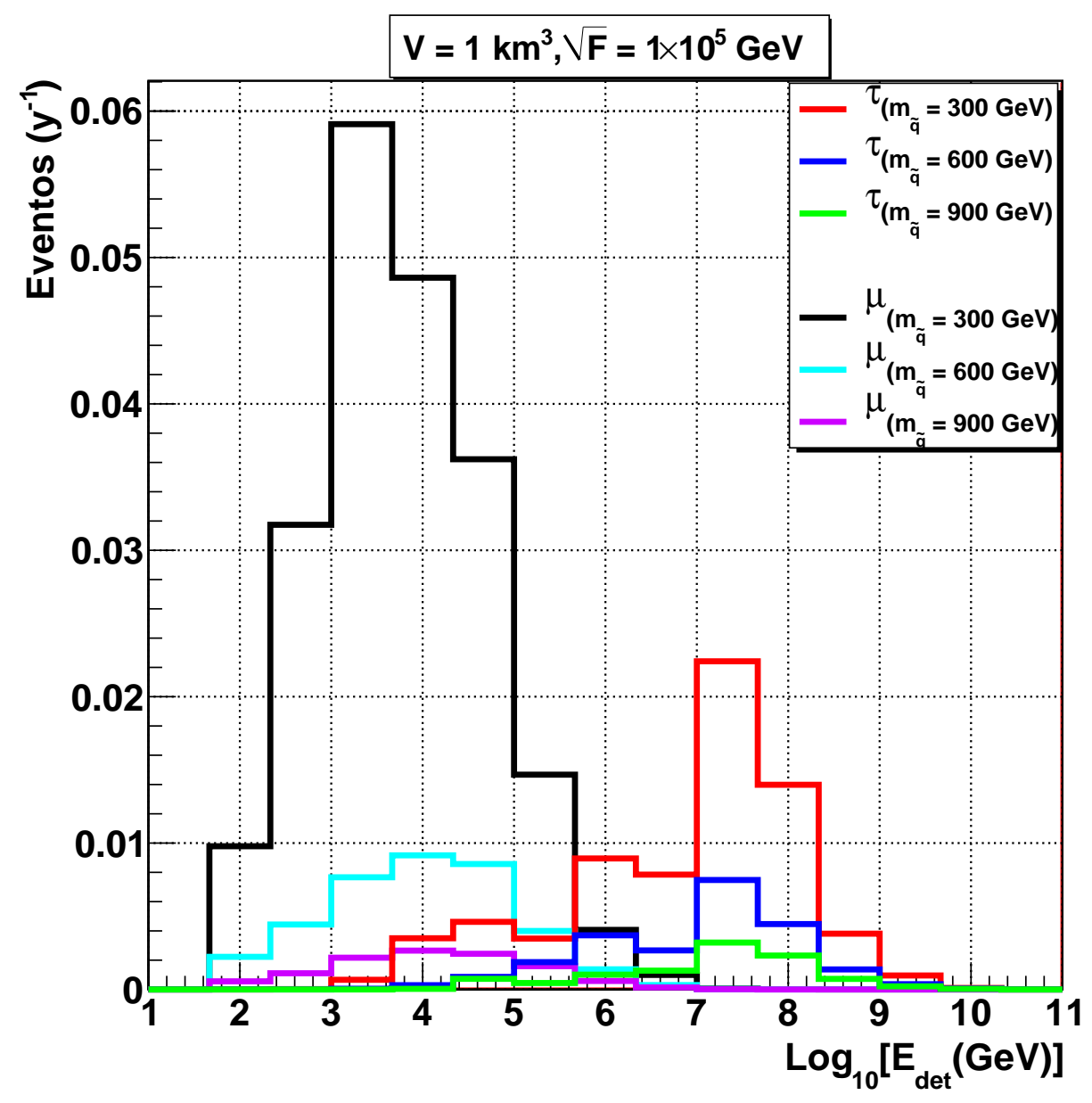

Figura 3.7: Distribuições de energia no detector para taus e múons, tomando-se três valores de massa squark produzido na interação neutrino-núcleon: 300, 600 e $900 \mathrm{GeV} \cdot \sqrt{F}=1 \times 10^{5} \mathrm{GeV}$. 


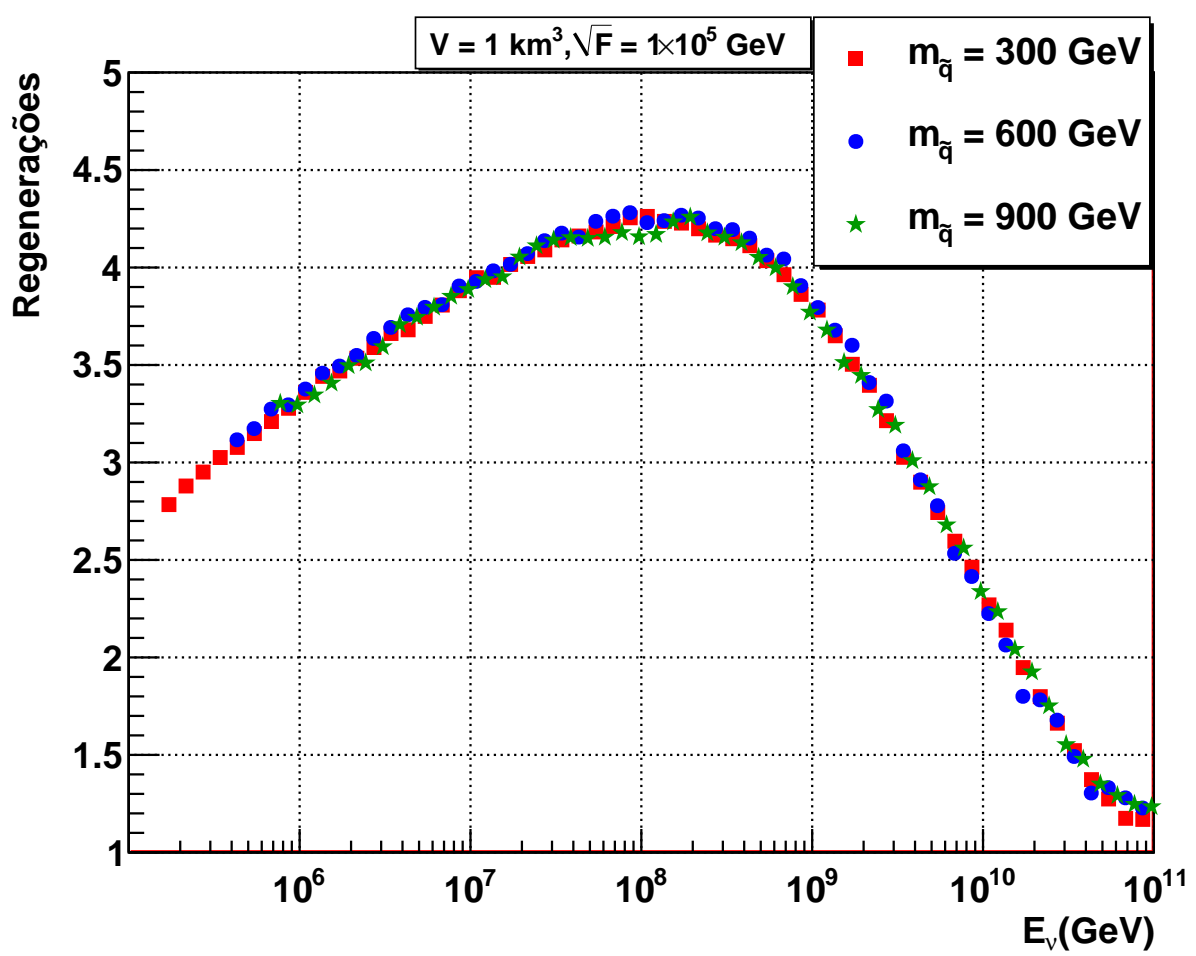

Figura 3.8: Número de regenerações médio do tau, relativas aos taus que atingem o detector, em função da energia do neutrino incidente, para $\sqrt{F}=1 \times 10^{5} \mathrm{GeV}$ e três valores de $m_{\tilde{q}}: 300,600$ e $900 \mathrm{GeV}$.

Como mencionado no início desta seção, os resultados apresentados acima foram obtidos para diversos valores de $\sqrt{F}$. De forma geral o comportamento dos gráficos obtidos para os outros valores deste parâmetro são similares. Na figura 3.9 apresentamos o número total de eventos por ano no volume do detector, para taus, múons e staus e na figura 3.10 temos os valores médios das energias com as quais as partículas chegam ao detector, ambas em função de $\sqrt{F}$. As barras de incerteza nos gráficos correspondem aos desvios-padrão das distribuições, representando as larguras das mesmas. Nos casos dos taus e múons os valores máximos de $\sqrt{F}$ são limitados aos quais os números de eventos são estatisticamente viáveis para as simulações (vide figura 3.9). 

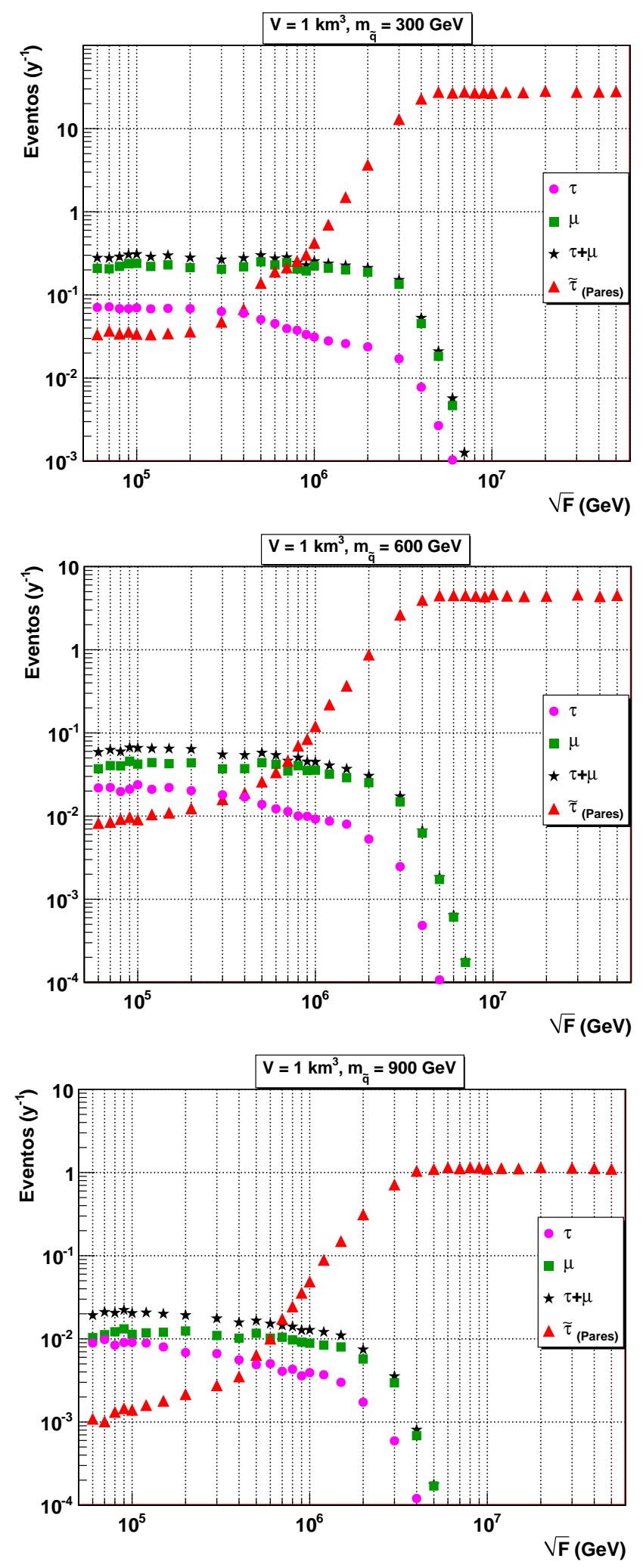

Figura 3.9: Fluxos de partículas por ano no volume de um telescópio de neutrinos de $1 \mathrm{~km}^{3}$, como o IceCube, em função de $\sqrt{F}$. 

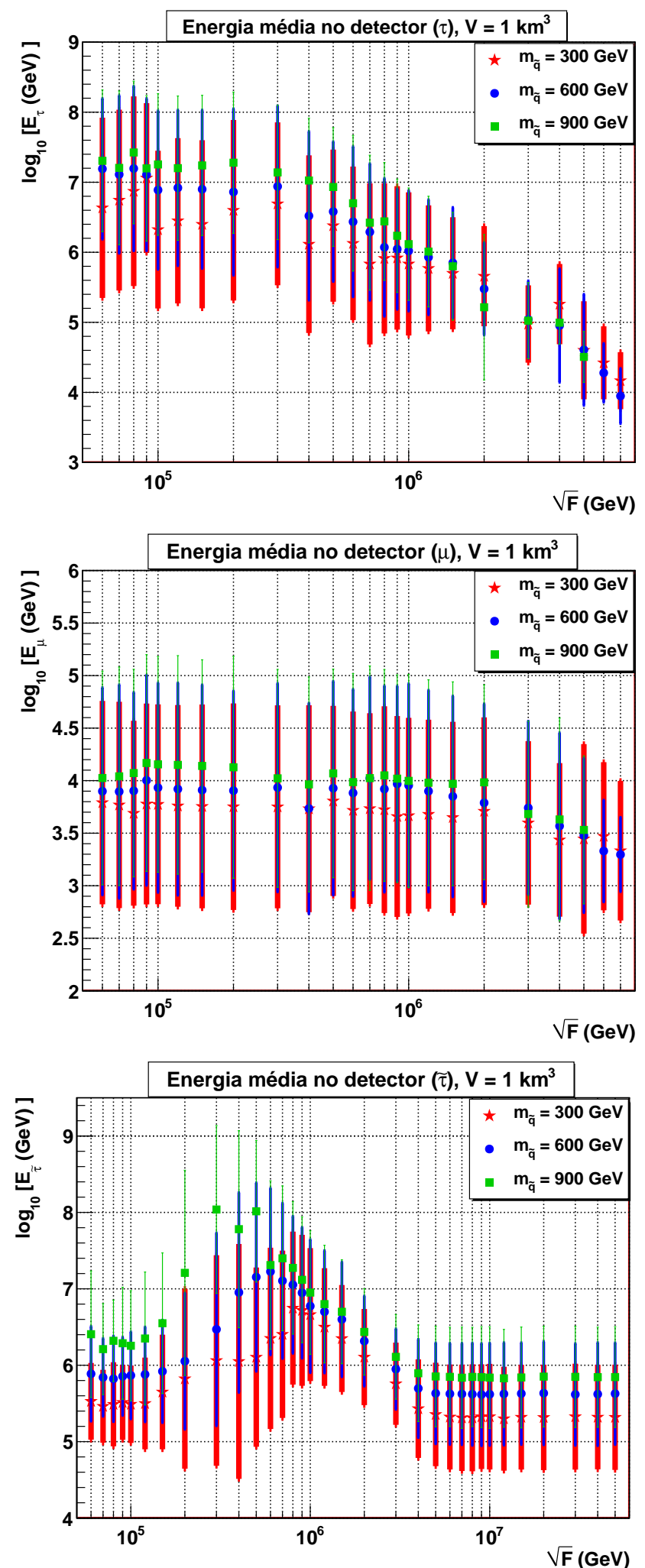

Figura 3.10: Energia média de taus, múons e staus (de cima para baixo) chegando no volume de um telescópio de neutrinos de $1 \mathrm{~km}^{3}$. As barras de incerteza correspondem aos desvios-padrão das distribuições. 
Nas curvas apresentadas na figura 3.9 nota-se uma queda suave no número de taus até cerca de $10^{6} \mathrm{GeV}$, sendo seguida de uma queda acentuada. No caso dos múons pode-se dizer que há um platô até cerca de $10^{6} \mathrm{GeV}$, sendo também seguido de uma queda acentuada. Em ambos os casos a diminuição do número de eventos é devida ao aumento do número de NLSPs que não decaem na Terra. No caso dos staus o comportamento é diferente, tendo-se poucos eventos para valores mais baixos de $\sqrt{F}$ e um crescimento acentuado no número de eventos a partir de cerca de $4 \times 10^{5} \mathrm{GeV}$, atingindo um valor máximo a partir de cerca de $5 \times 10^{6} \mathrm{GeV}$, que representa o valor de $\sqrt{F}$ para o qual as NLSPs são estáveis durante sua passagem pela Terra. O número de eventos de staus não tende a zero para os menores valores de $\sqrt{F}$ porque há um número mínimo que é produzido dentro do detector, ou seja, a interação neutrino-núcleon ocorre dentro do volume de detecção. Assim, eles são contabilizados pela simulação mesmo que decaiam instantaneamente.

Para o caso da figura 3.10 há também um comportamento muito similar entre as curvas obtidas para os três valores da massa do squark. Entretanto, a energia média no detector é sistematicamente mais alta para valores maiores desta massa, pois o limiar para a energia de produção de NLSPs é maior. Para os taus há uma queda no valor médio da energia, que é mais significativa para $\sqrt{F}$ a partir de cerca de $4 \times 10^{5} \mathrm{GeV}$. Já no caso dos múons, isto só ocorre a partir de cerca de $3 \times 10^{6} \mathrm{GeV}$. Para ambos os casos esta queda na energia média ocorre porque à medida em que $\sqrt{F}$ aumenta somente as NLSPs de menor energia decaem dentro da Terra (figuras 3.1 e 3.2). Os staus apresentam um comportamento distinto: há um máximo no valor da energia média para $\sqrt{F}$ a cerca de $5 \times 10^{5} \mathrm{GeV}$. A partir de $\sqrt{F}$ aproximadamente igual a $5 \times 10^{6} \mathrm{GeV}$, quando o número de decaimentos dentro da Terra já é muito pequeno, o valor médio da energia passa a ser constante. Esse máximo ocorre porque para valores mais baixos de $\sqrt{F}$, para os quais há um número grande de decaimentos dentro da Terra, os staus que atingem o detector são os mais energéticos (figura 3.2), pois são os que conseguem percorrer maiores distâncias antes de decair.

De forma geral, as taxas obtidas par o IceCube são relativamente baixas. Um dos motivos para isto é a extensão da região na qual o tau pode ser produzido de forma a ser passível de detecção, que é muito restrita, pois esta partícula decai muito rapidamente. Observando a figura 3.3 nota-se 
que o tau só passa a percorrer uma distância mais significativa, comparada às dimensões do detector, a partir de $10^{7} \mathrm{GeV}$, tanto que a distribuição de energia obtida (figura 3.7) se concentra nesta faixa de energia. Desta forma a partícula deve ser produzida no volume do detector ou a uma distância muito pequena dele para que seja detectável. No caso do múon, obtemos mais eventos porque esta partícula não decai tão rapidamente. Mesmo ela perdendo mais energia em sua trajetória, o que é refletido na distribuição de energia obtida (figura 3.7), que fica centrada torno de $10^{4} \mathrm{GeV}$, esta partícula pode percorrer algumas dezenas de quilômetros antes de perder toda sua energia (figura 2.14).

Outra contribuição para o baixo número de taus chegando ao telescópio de neutrinos de $1 \mathrm{~km}^{3}$, associada indiretamente à alta probabilidade de decaimento do tau, é a degradação da energia do tau (e do neutrino do tau) durante os ciclos de regeneração desta partícula. Todas as vezes em que o tau decai, produzindo um neutrino do tau, a probabilidade de sobrevivência deste novo neutrino aumenta (figura 2.5), pois a chance dele interagir com um núcleon no percurso restante até a região do detector diminui. A cada regeneração este percurso se torna menor e a energia do neutrino do tau produzido no decaimento também. Com isso, a grande maioria dos neutrinos do tau acabam passando pelo detector sem produzir um tau em seu volume, e quando este é produzido a probabilidade de decaimento é cada vez maior. 


\subsubsection{Taus de decaimentos de NLSPs - IceCube ampliado}

Dado que o número de eventos obtidos para o telescópio de neutrinos de $1 \mathrm{~km}^{3}$ é relativamente baixo e que há propostas de ampliação do telescópio IceCube [26], determinamos os fluxos de taus e múons provenientes do decaimento de NLSPs para dois volumes adicionais: para um aumento médio de $2 \mathrm{~km}$ no raio do IceCube e para a maior configuração abordada em [26], com aumento no raio do detector de $4 \mathrm{~km}$. A profundidade do detector é sempre mantida em cerca de $2 \mathrm{~km}$, sendo os volumes resultantes de cerca de 21 e 65 $\mathrm{km}^{3}$, respectivamente.

Nas figuras 3.11 a 3.13 são apresentados os espectros de energia obtidos para estes dois volumes de telescópios de neutrinos. As formas das curvas são similares às obtidas anteriormente para um volume de $1 \mathrm{~km}^{3}$, no entanto há um aumento significativo do fluxo das partículas com o aumento do volume.

Na na tabela 3.3 são apresentadas as taxa totais de eventos. Vale lembrar que como no caso de $1 \mathrm{~km}^{3}$, as curvas obtidas para os taus e múons correspondem a apenas um stau do par de staus produzido. Para todas as massas do squark temos uma quantidade significativa de eventos já para um aumento de $2 \mathrm{~km}$ no raio do detector. Nota-se também que o aumento no número de taus é proporcionalmente maior do que o de múons. Isto é uma consequência direta do aumento do volume do detector, pois para energias mais baixas, para as quais o tau percorre menores distâncias, há um ganho

maior. É possível observar que o aumento no número de taus é aproximadamente o mesmo aumento observado no volume enquanto que para os múons, é aproximadamente metade.

Nas figuras 3.14 e 3.15 são apresentadas as respectivas distribuições de energia. Nota-se que o comportamento médio das distribuições de energia não muda significativamente com o aumento do volume do detector, aumentandose apenas o número de eventos e o aumento relativo da taxa de taus frente à de múons.

O efeito do aumento do volume do detector na taxa de eventos total é ilustrado também na figura 3.17, onde são mostradas apenas as somas das taxas de taus com as de múons e também as de pares de staus. 

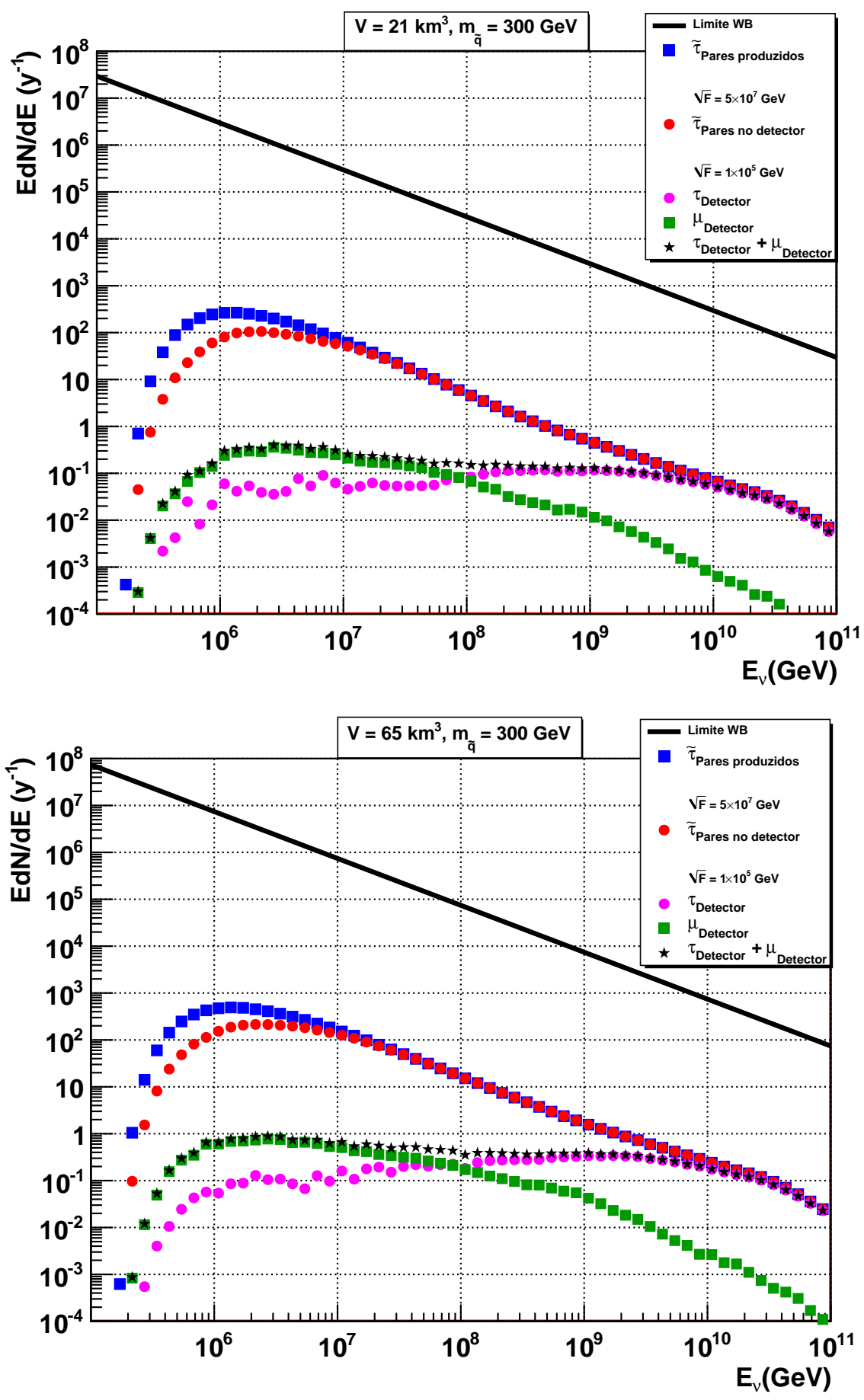

Figura 3.11: Fluxo de taus para telescópios de neutrinos de raios $2 \mathrm{~km}$ (acima) e 4 $\mathrm{km}$ (abaixo) maiores do que o IceCube. A massa do squark produzido na interação neutrino-núcleon é $300 \mathrm{GeV}$. 

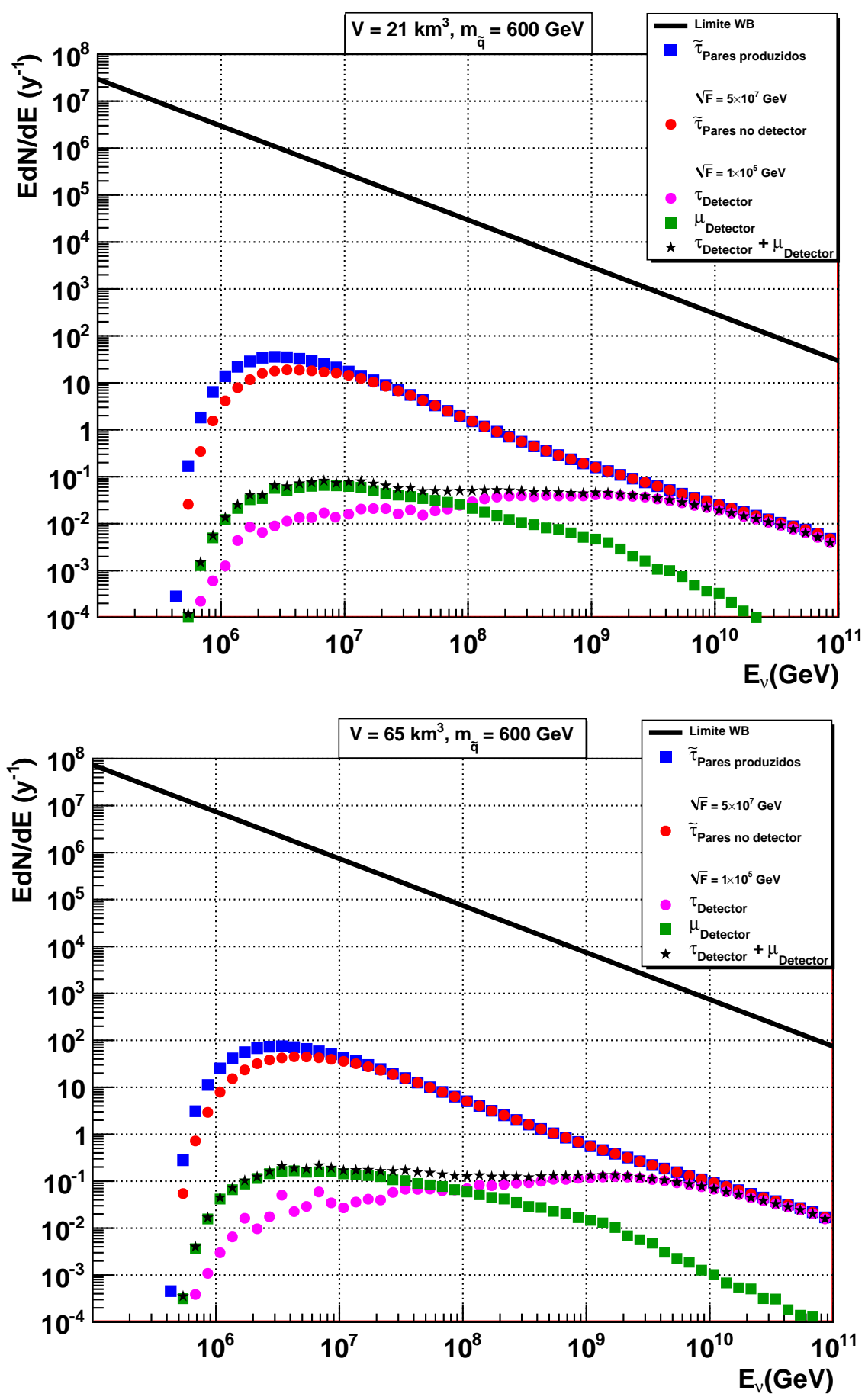

Figura 3.12: Fluxo de taus para telescópios de neutrinos de raios $2 \mathrm{~km}$ (acima) e 4 km (abaixo) maiores do que o IceCube. A massa do squark produzido na interação neutrino-núcleon é $600 \mathrm{GeV}$. 

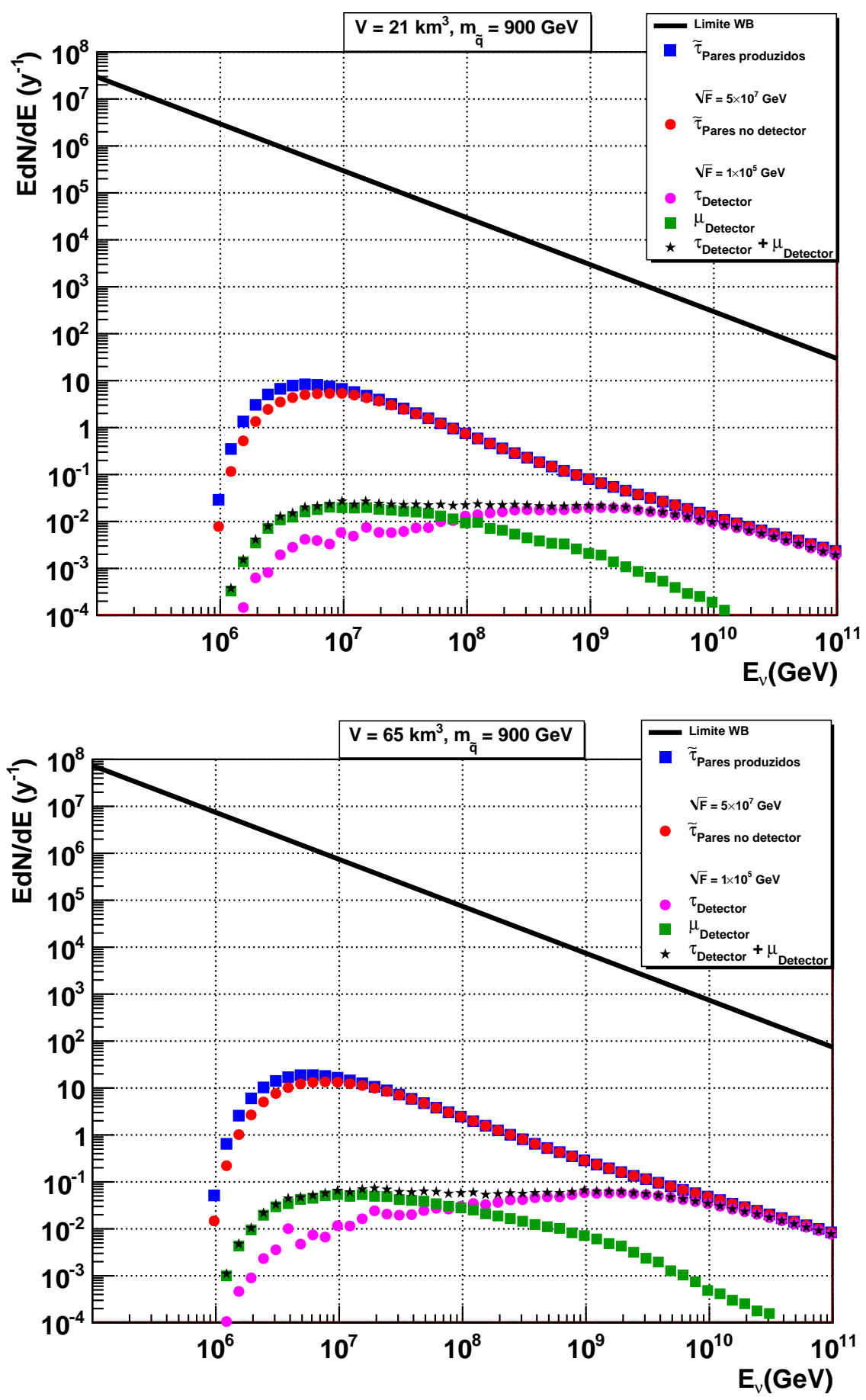

Figura 3.13: Fluxo de taus para telescópios de neutrinos de raios $2 \mathrm{~km}$ (acima) e 4 km (abaixo) maiores do que o IceCube. A massa do squark produzido na interação neutrino-núcleon é $900 \mathrm{GeV}$. 
Tabela 3.3: Taxas de eventos por ano para os volumes e massas do squark especificados. Para staus, $\sqrt{F}=5 \times 10^{7} \mathrm{GeV}$; para taus e múons, $1 \times 10^{5} \mathrm{GeV}$.

\begin{tabular}{|c|c|c|c|c|c|}
\hline $\mathrm{m}_{\tilde{q}}(\mathrm{GeV})$ & $\mathrm{V}\left(\mathrm{km}^{3}\right)$ & $\tilde{\tau}\left(\mathrm{y}^{-1}\right)$ & $\tau\left(\mathrm{y}^{-1}\right)$ & $\mu\left(\mathrm{y}^{-1}\right)$ & $\mu+\tau\left(\mathrm{y}^{-1}\right)$ \\
\hline 300 & 1 & 27.7 & 0.07 & 0.21 & 0.28 \\
300 & 21 & 521.5 & 1.60 & 2.36 & 3.95 \\
300 & 65 & 1450.5 & 4.41 & 5.85 & 10.26 \\
\hline 600 & 1 & 4.5 & 0.02 & 0.04 & 0.06 \\
600 & 21 & 100.6 & 0.51 & 0.47 & 0.98 \\
600 & 65 & 260.0 & 1.47 & 1.27 & 2.74 \\
\hline 900 & 1 & 1.1 & 0.01 & 0.01 & 0.02 \\
900 & 21 & 28.2 & 0.22 & 0.15 & 0.37 \\
900 & 65 & 73.0 & 0.65 & 0.41 & 1.05 \\
\hline
\end{tabular}

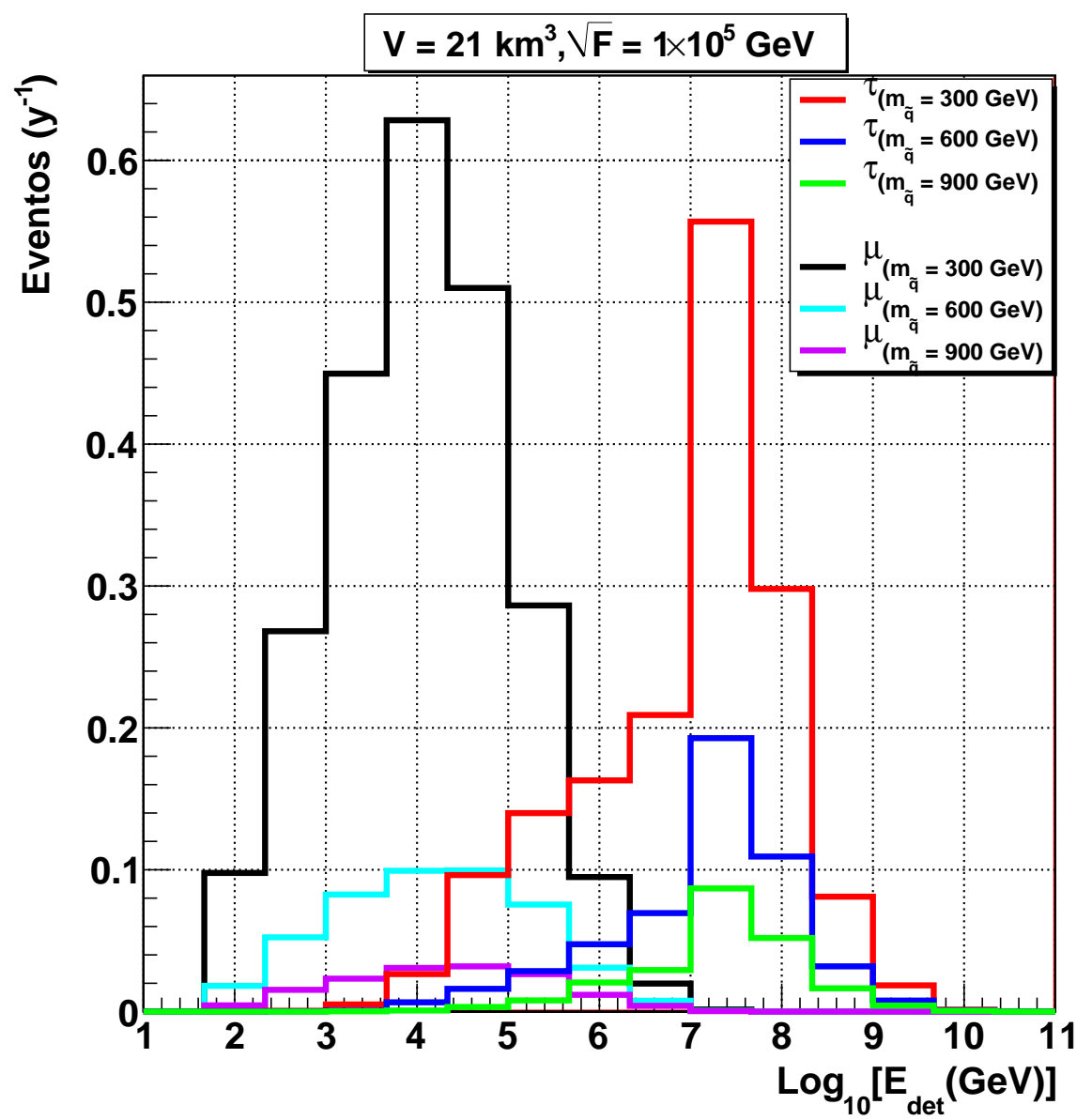

Figura 3.14: Distribuições de energia no detector para taus e múons, com $\sqrt{F}=$ $1 \times 10^{5} \mathrm{GeV}$. 


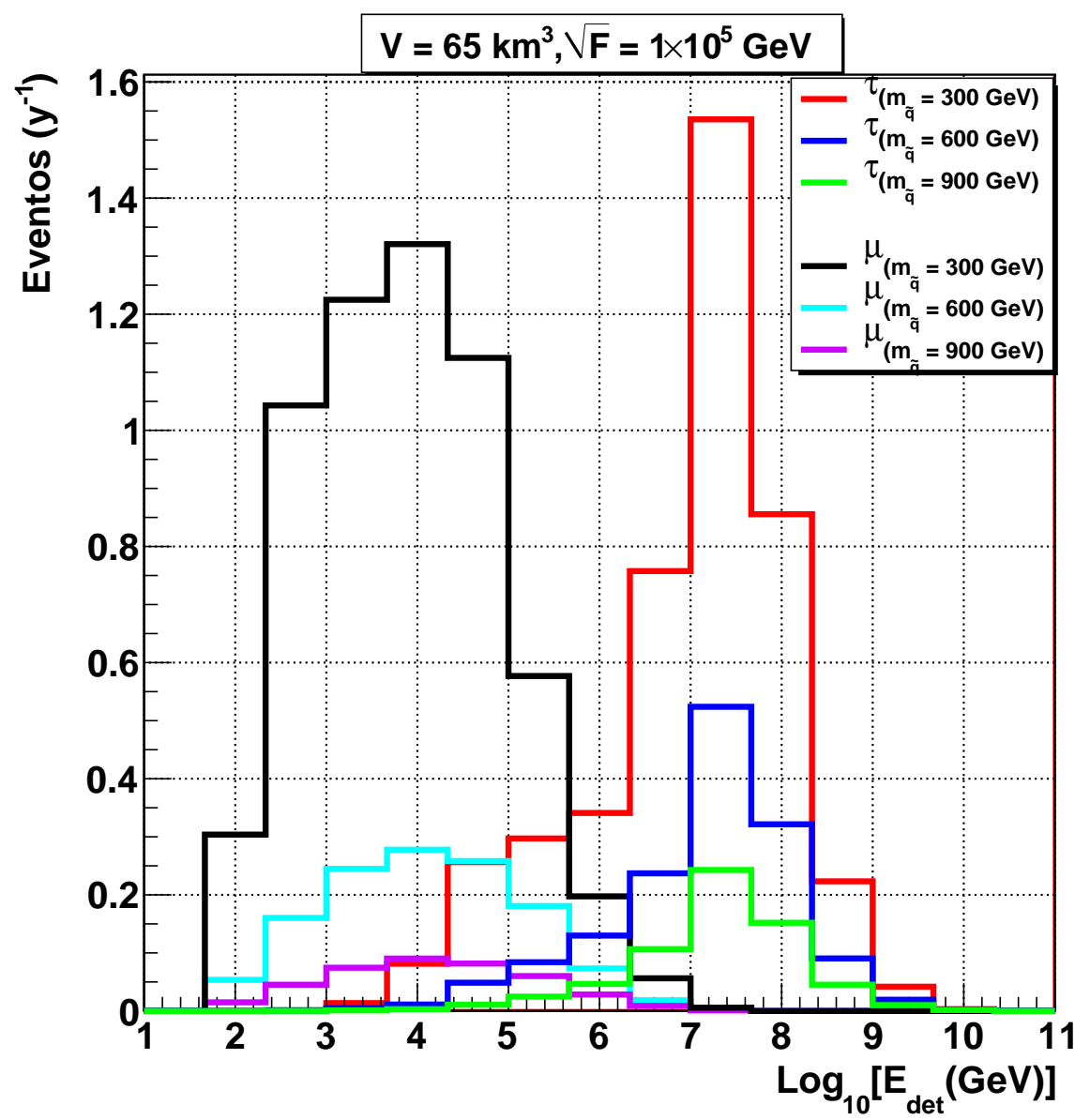

Figura 3.15: Distribuições de energia no detector para taus e múons, para três valores de massa squark produzido na interação neutrino-núcleon: 300, 600 e 900 $\mathrm{GeV} \cdot \sqrt{F}=1 \times 10^{5} \mathrm{GeV}$.

Em relação às regenerações do tau, notamos que o número médio diminui com o aumento do volume do detector (figura 3.16), o que é esperado visto que o tau pode ser detectado em uma região maior.

Como no caso de $1 \mathrm{~km}^{3}$, nas figuras 3.18 a 3.20 temos gráficos do número de eventos em função de $\sqrt{F}$. De forma geral, o comportamento das curvas é similar aos apresentados na seção anterior, porém com muito mais eventos, o que proporciona taxas de taus ou múons em quantidades significativas para valores maiores de $\sqrt{F}$. O comportamento do valor médio da energia em função de $\sqrt{F}$ é equivalente aos apresentados na figura 3.10. 


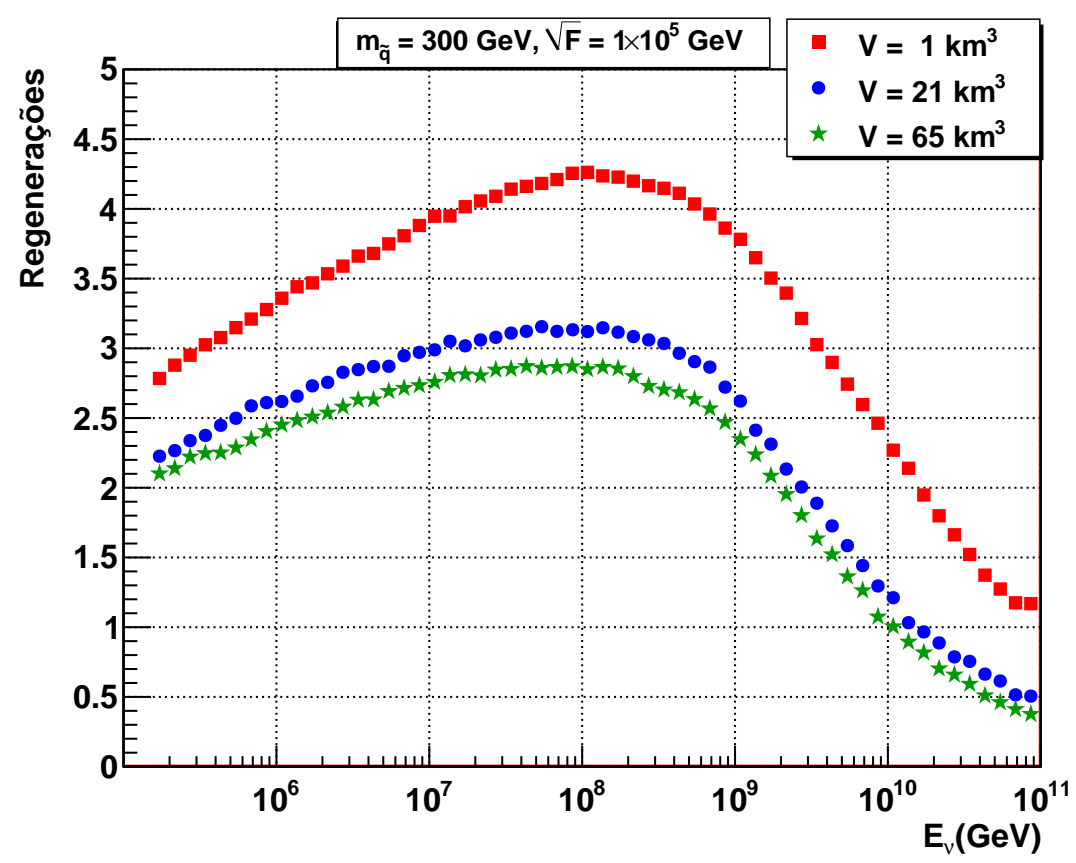

Figura 3.16: Regenerações do tau em função da energia do neutrino incidente para 3 volumes de telescópio de neutrinos, para a massa do squark de $300 \mathrm{GeV}$. 

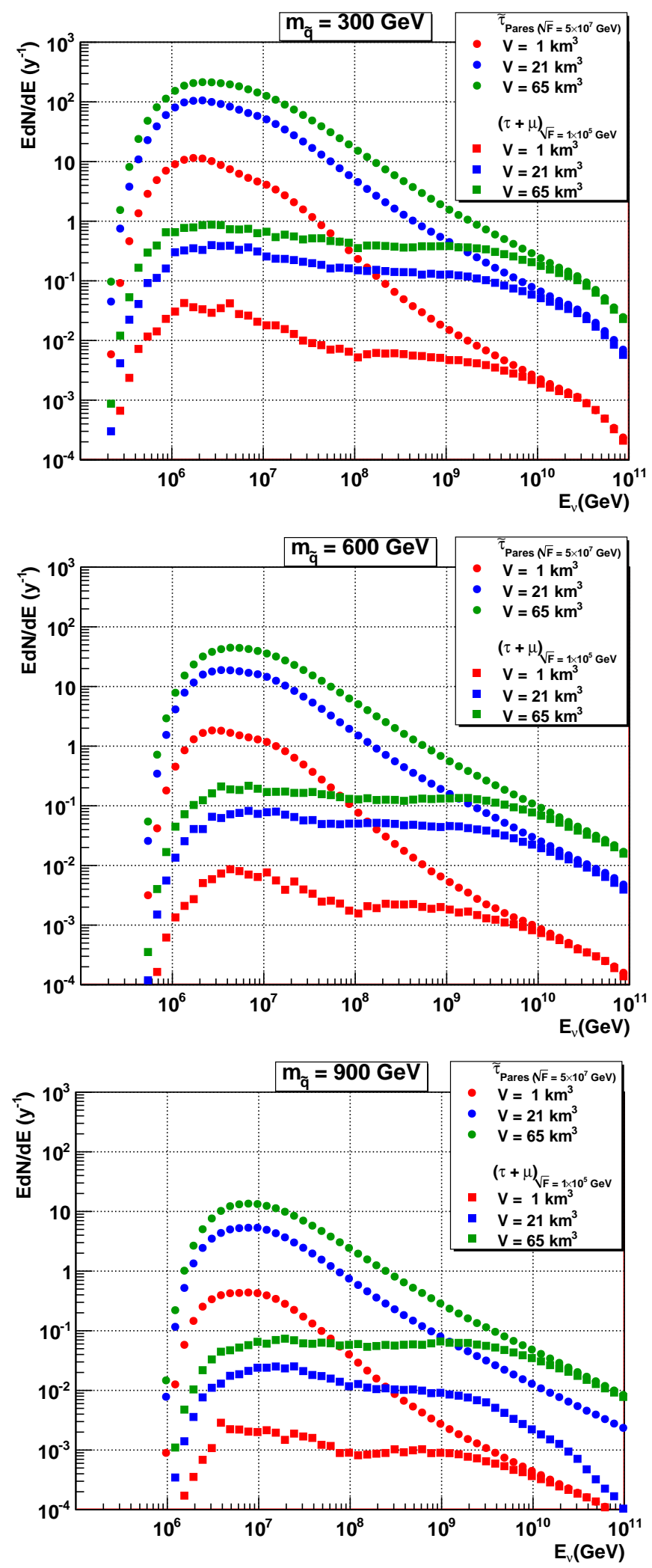

Figura 3.17: Fluxo de staus e de taus mais múons em 3 telescópios de neutrinos de diferentes volumes. 

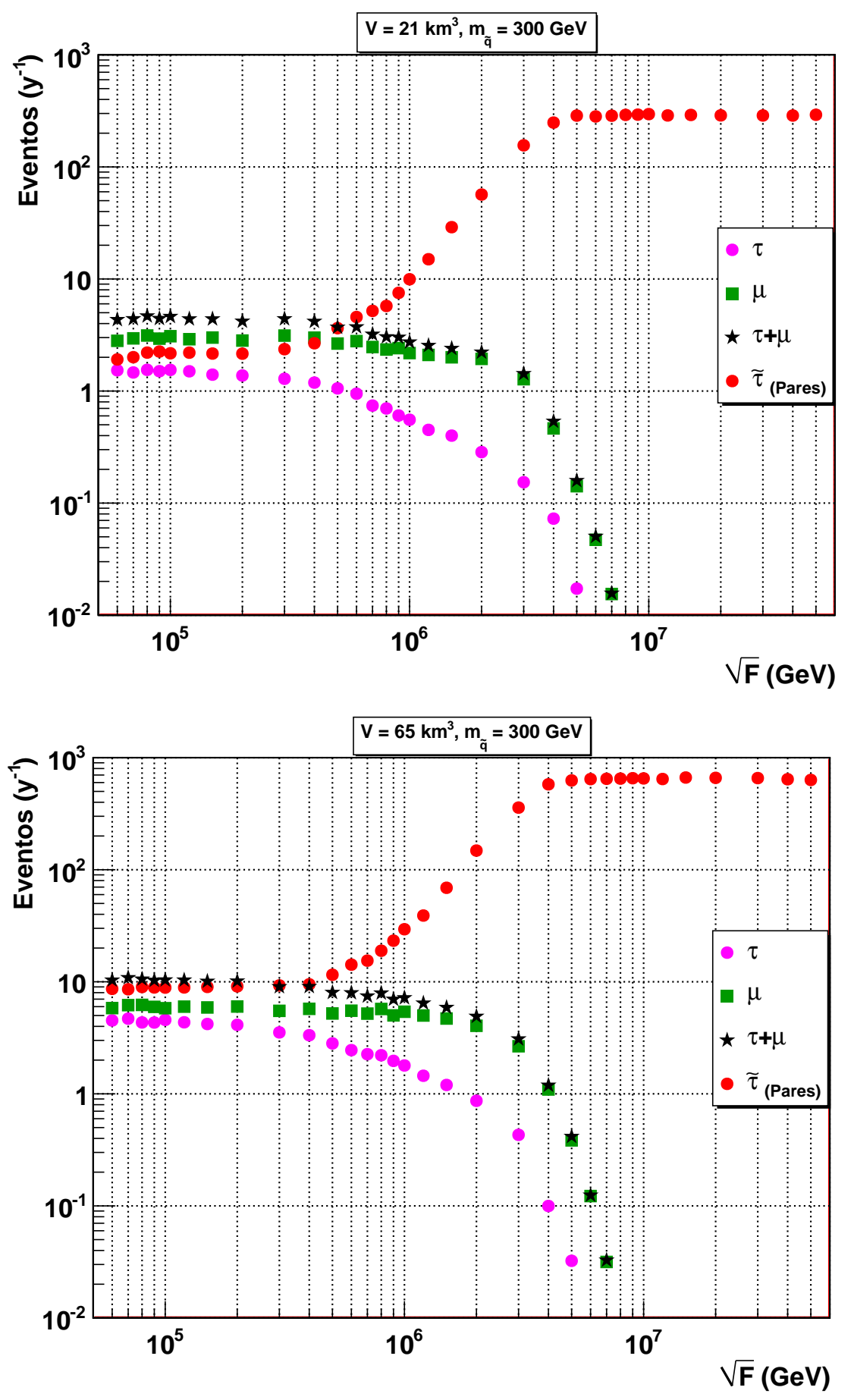

Figura 3.18: Fluxos de partículas por ano em telescópios de neutrinos similares ao IceCube, porém com raios $2 \mathrm{~km}$ (acima) e $4 \mathrm{~km}$ (abaixo) maiores, para $m_{\tilde{q}}=300$ $\mathrm{GeV}$. 

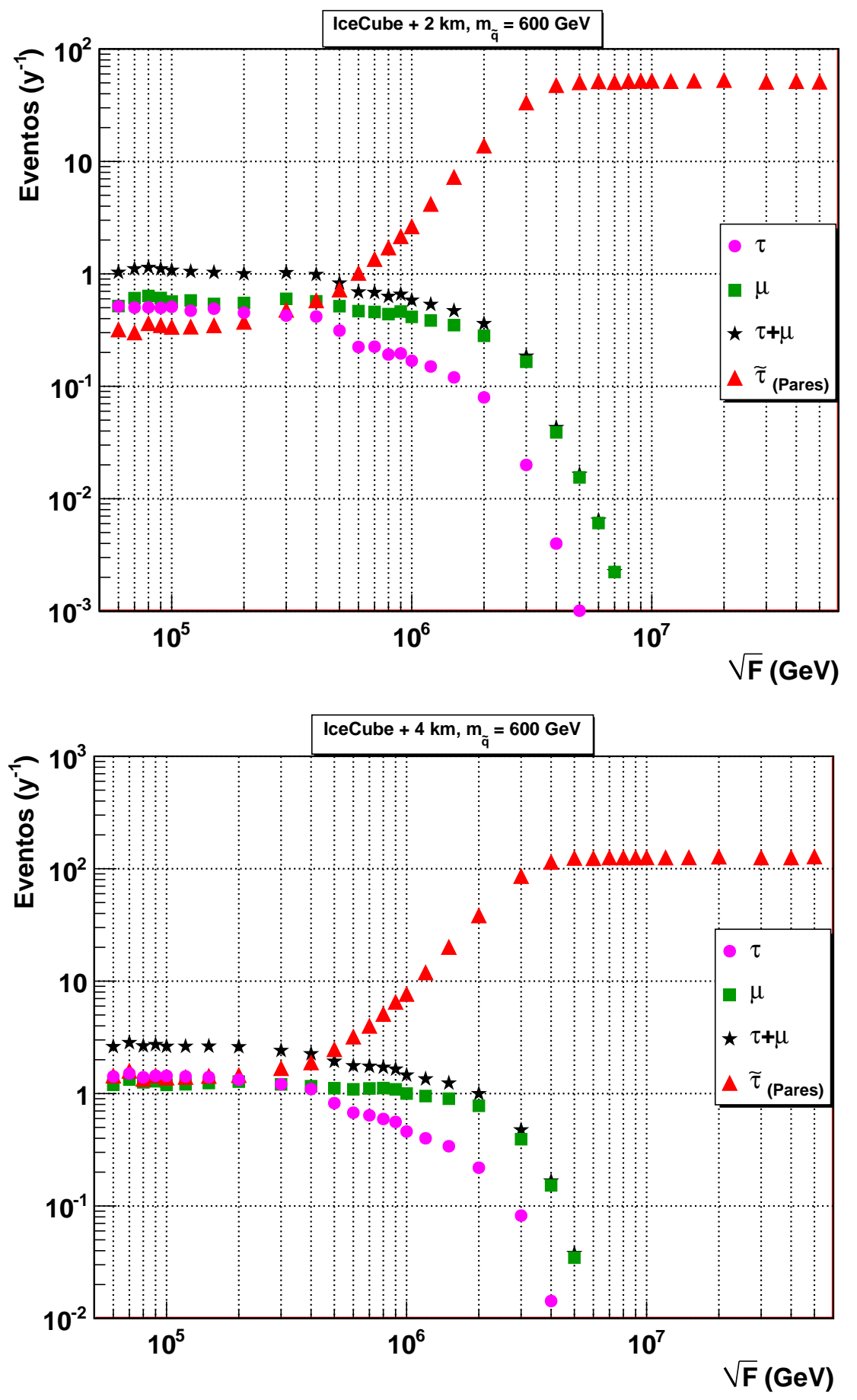

Figura 3.19: Fluxos de partículas por ano em telescópios de neutrinos similares ao IceCube, porém com raios $2 \mathrm{~km}$ (acima) e $4 \mathrm{~km}$ (abaixo) maiores, para $m_{\tilde{q}}=600$ $\mathrm{GeV}$. 

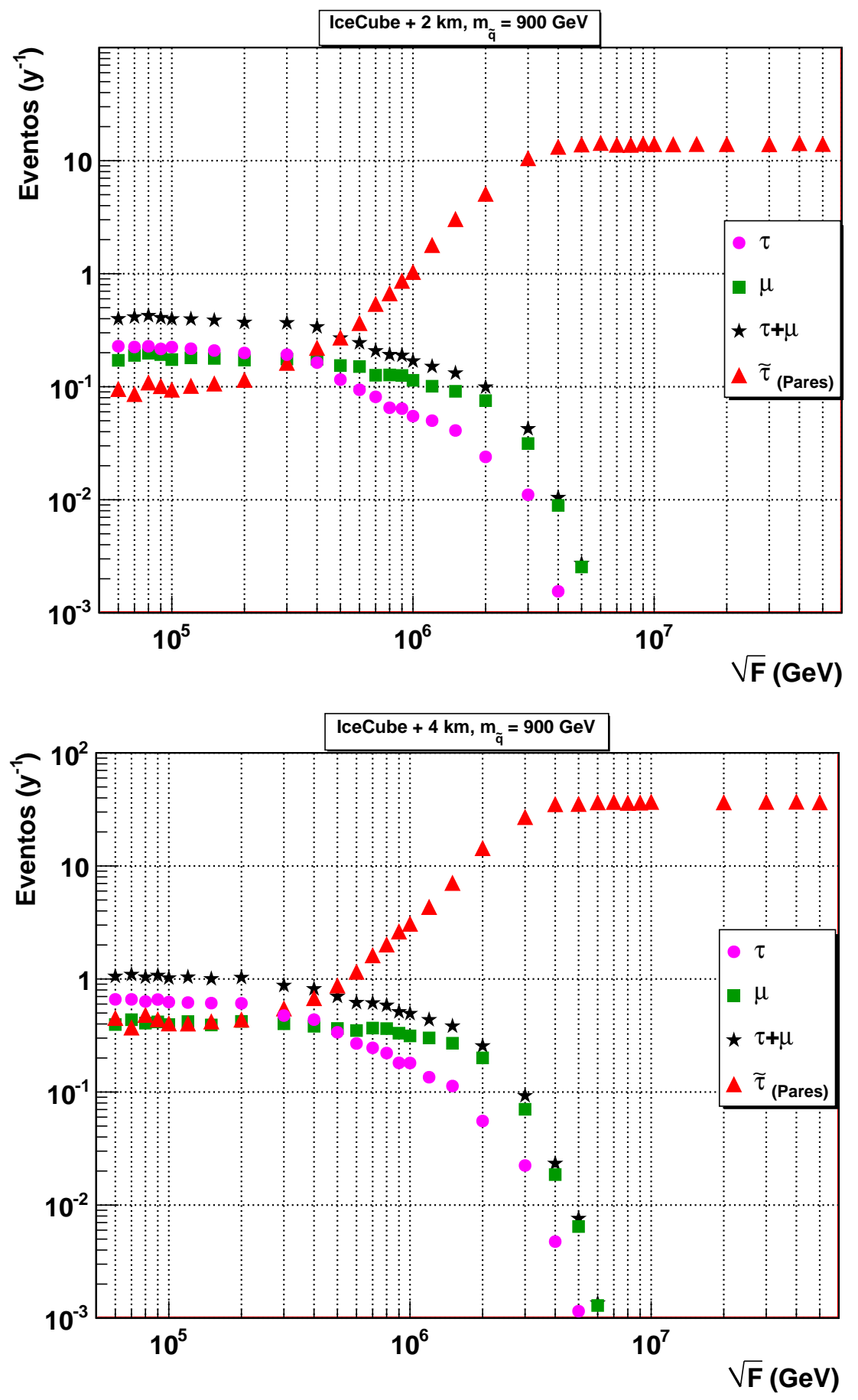

Figura 3.20: Fluxos de partículas por ano em telescópios de neutrinos similares ao IceCube, porém com raios $2 \mathrm{~km}$ (acima) e $4 \mathrm{~km}$ (abaixo) maiores, para $m_{\tilde{q}}=900$ $\mathrm{GeV}$. 


\subsubsection{Produção direta de taus}

O fato de o tau decair quase que instantaneamente, dependendo de sua energia, implica em sinais característicos em telescópios de neutrinos [27]. Uma das assinaturas de maior destaque é um sinal conhecido como Double Bang [32], onde devem ocorrer dois eventos dentro do volume do detector. O primeiro seria um chuveiro hadrônico $(X)$ produzido na interação de corrente carregada do neutrino do tau, $\nu_{\tau}+N \rightarrow \tau+X$. O tau produzido nesta interação precisa percorrer cerca de $100 \mathrm{~m}$ dentro do detector antes de decair, produzindo um segundo chuveiro hadrônico. Esta distância permite que os dois eventos sejam distinguíveis dentro detector. A energia necessária para produzir um tau com estas características [32] é de $10^{6} \mathrm{GeV}$, como pode ser observado na figura 3.3: para energias acima de $2 \times 10^{6} \mathrm{GeV}$ o tau passa a percorrer, em média, distâncias acima de $100 \mathrm{~m}$ antes de decair, com uma probabilidade menor de observar o segundo evento dentro do detector. Outras assinaturas características do tau são descritas adiante.

Com o intuito de verificar a consistência de nossa simulação da regeneração do tau, simulamos a produção de taus por meio da interação de neutrinos cosmológicos com núcleons da Terra e comparamos nossos resultados com outros que analisam a detecção de taus. Além de eventos do tipo Double Bang, analisamos as seguintes assinaturas características do tau [27] e também descrevemos os critérios que adotamos em nossa simulação:

Lollipop : relativos aos taus que são produzidos antes de chegar ao detector e decaem dentro dele. Neste caso consideramos os taus que são produzidos a pelo menos $300 \mathrm{~m}$ do detector e que percorrem pelo menos 200 $m$ dentro dele antes de decair, para que se tenha uma assinatura clara da trajetória. Além disso o decaimento precisa ocorrer a pelo menos $125 \mathrm{~m}$ da borda do detector, para garantir que pelo menos um conjunto de fotomultiplicadoras seja atingido;

Inverted Lollipop (InvLollipop) : correspondem a taus que são produzidos dentro do volume do detector e decaem após saírem dele. Neste caso os taus também precisam percorrer pelo menos $200 \mathrm{~m}$ dentro do detector antes de decair, para caracterizar bem a assinatura causada pela perda de energia; 
Tautsipop : taus que são produzidos como também decaem dentro do volume do detector, de forma que o ponto de produção e o ponto de decaimento não possam ser distinguíveis. Neste caso foram tomados os decaimentos que ocorrem com menos de $50 \mathrm{~m}$. Após o decaimento também é necessário que haja 125 m até a borda para que as partículas do decaimento possam ser observadas.

As assinaturas Lollipop, InvLollipop e Double Bang ocorrem em $82 \%$ dos decaimentos do tau, excluindo-se os decaimentos que resultam em múons, para os quais temos eventos do tipo Tautsipop (18\%).

Na figura 3.21 apresentamos as frações de eventos em função da energia do neutrino incidente para diferentes assinaturas do tau, tomando como referência os três volumes de telescópios de neutrinos abordados nas seções anteriores. Nela são apresentados também o número de total de taus que atingem o detector e os taus que são produzidos antes de atingi-lo (a $300 \mathrm{~m}$ ) e passam pelo seu volume sem decair.

Na tabela 3.4 são apresentadas as taxas de eventos por ano para os três volumes considerados. Esta taxa é dada independentemente do fluxo de neutrinos, ou seja, como a fração de eventos gerados, bem como para fluxos correspondentes aos impostos pelos limites WB, MPR para fontes finas e MPR para fontes espessas ${ }^{6}$. Nota-se que à medida em que o volume do detector aumenta as proporções de cada contribuição muda. Por exemplo os eventos do tipo Double Bang passam a ter uma representação proporcionalmente maior. Nota-se que mesmo para o limite mais conservador (WB), o número de eventos do tipo Double Bang é significativo. Porém devemos levar em conta que o aumento do volume pode implicar no aumento da energia mínima aceita pelo detector. Dependendo da configuração adotada uma parte significativa dos eventos do tipo Double Bang, por exemplo, poderia não ser observada se o limiar de detecção for muito maior do que o do IceCube atual $(\sim 100$ $\mathrm{GeV})$.

\footnotetext{
${ }^{6}$ Nas pesquisas [6, 7] também foi avaliado o uso do limite de Mannheim, Protheroe e Rachen (MPR) [13]. Usamos este limite apenas nesta seção para compararmos nossos resultados da simulação da regeneração do tau com resultados já existentes.
} 

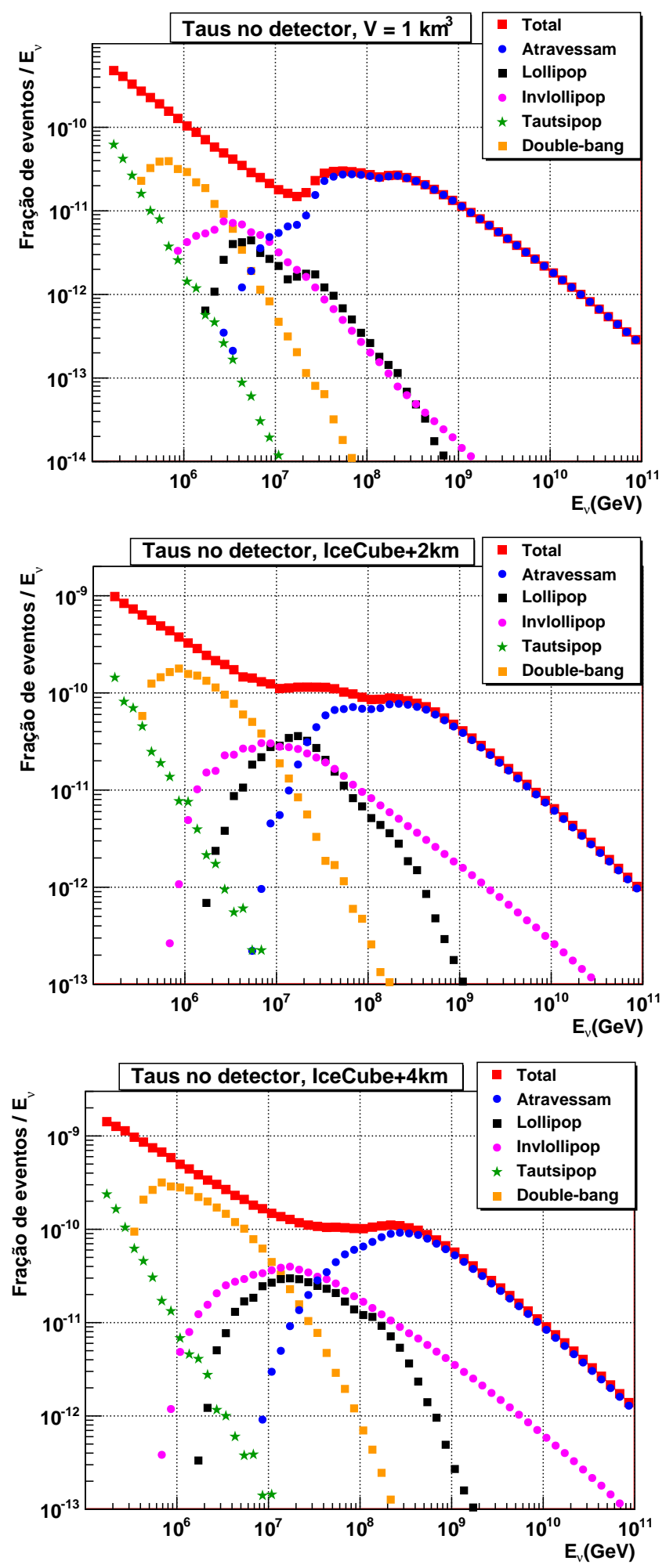

Figura 3.21: Fração de eventos de taus em função da energia do neutrino incidente. São apresentados também a soma de todos os taus que atingem o detector e aqueles que passam por ele sem decair. Os outros casos estão descritos no texto. 
Nossos resultados são compatíveis com os obtidos em [28] onde o limiar de energia é de $2 \times 10^{6} \mathrm{GeV}$. Os fluxos considerando este limiar são apresentados na tabela 3.5 .

Tabela 3.4: Número de taus por ano em telescópios de neutrinos de 3 volumes, normalizados com 3 diferentes limites para o fluxo de neutrinos: WB, $\mathrm{MPR}_{f}$ (fontes finas) e $\mathrm{MPR}_{e}$ (fontes espessas). A taxa de eventos é apresentada independentemente do fluxo de neutrinos, ou seja, a fração $f_{N}$ dos eventos gerados que chegam ao detector, bem como para as diferentes assinaturas do tau: Lollipop, InvLollipop, Tautsipop e Double Bang. Além disso, são apresentadas as taxas para o total de taus e para os taus que atravessam o volume do detector.

\begin{tabular}{|c|c|c|c|c|c|c|}
\hline \multicolumn{7}{|c|}{$\mathrm{V}=1 \mathrm{~km}^{3}$} \\
\hline Limite & Total & Lollipop & InvLollipop & Tautsipop & Atravessam & Double Bang \\
\hline$f_{N}$ & $3,1 \times 10^{-9}$ & $3,8 \times 10^{-11}$ & $7,8 \times 10^{-11}$ & $1,5 \times 10^{-10}$ & $4,9 \times 10^{-10}$ & $2,8 \times 10^{-10}$ \\
\hline WB & 124 & 1,5 & 3,1 & 6,0 & 19,5 & 11,3 \\
\hline $\mathrm{MPR}_{f}$ & 1098 & 4,3 & 9,2 & 456 & 34,9 & 88 \\
\hline $\mathrm{MPR}_{e}$ & 4660 & 69 & 116 & 1250 & 730 & 520 \\
\hline \multicolumn{7}{|c|}{$\mathrm{V}=21 \mathrm{~km}^{3}$} \\
\hline$f_{N}$ & $7,4 \times 10^{-8}$ & $2,9 \times 10^{-9}$ & $3,9 \times 10^{-9}$ & $3,0 \times 10^{-9}$ & $1,0 \times 10^{-8}$ & $1,4 \times 10^{-8}$ \\
\hline WB & 2930 & 114 & 156 & 120 & 460 & 550 \\
\hline $\mathrm{MPR}_{f}$ & 21720 & 270 & 350 & 8760 & 810 & 3520 \\
\hline $\mathrm{MPR}_{e}$ & 109900 & 5230 & 5860 & 24970 & 17130 & 25400 \\
\hline \multicolumn{7}{|c|}{$\mathrm{V}=65 \mathrm{~km}^{3}$} \\
\hline$f_{N}$ & $2,7 \times 10^{-7}$ & $8,3 \times 10^{-9}$ & $1,4 \times 10^{-8}$ & $1,3 \times 10^{-8}$ & $3,0 \times 10^{-8}$ & $6,4 \times 10^{-8}$ \\
\hline WB & 10640 & 328 & 547 & 496 & 1180 & 2530 \\
\hline $\mathrm{MPR}_{f}$ & 81950 & 770 & 1160 & 37100 & 2080 & 15700 \\
\hline $\mathrm{MPR}_{e}$ & 399100 & 15000 & 20500 & 103300 & 44200 & 116000 \\
\hline
\end{tabular}

Tabela 3.5: Idem à tabela 3.4, mas agora impondo um limiar de energia de $2 \times 10^{6}$ $\mathrm{GeV}$.

\begin{tabular}{|c|c|c|c|c|c|c|}
\hline Limite & Total & Lollipop & InvLollipop & Tautsipop & Atravessam & Double Bang \\
\hline$f_{N}$ & $7,9 \times 10^{-10}$ & $3,7 \times 10^{-11}$ & $5,6 \times 10^{-11}$ & $9,4 \times 10^{-13}$ & $4,9 \times 10^{-10}$ & $3,1 \times 10^{-11}$ \\
\hline WB & 31 & 1,5 & 2,2 & $3,7 \times 10^{-2}$ & 19,5 & 1,3 \\
\hline MPR $_{f}$ & 65 & 4,1 & 5,4 & 0,6 & 34,9 & 4,4 \\
\hline MPR $_{e}$ & 1170 & 67 & 83 & 7,8 & 730 & 57 \\
\hline
\end{tabular}

Na figura 3.22 são apresentadas as distribuições de energia, normalizadas pelas frações de eventos $\left(f_{N}\right)$ apresentadas na tabela 3.4 , para cada tipo de eventos de taus. Nos três gráficos é possível identificar parcialmente as 
contribuições das diferentes assinaturas do tau no detector em relação ao total de eventos, pois o total não corresponde à soma das assinaturas apresentadas. Para energias menores muitos eventos não satisfazem as condições impostas de distância percorrida pelo tau antes de decair. Contudo fica claro que os taus que atravessam o detector dominam a região de mais altas energias. Apresentamos também, na figura 3.23, as mesmas distribuições de energia sem a distribuição do total de eventos, para ilustrar melhor as distribuições de energia de cada tipo de evento no detector, pois ficam suprimidas na figura 3.22 .

É interessante registrar também o número de regenerações do tau para o fluxo de total de taus no detector. Isto é mostrado na figura 3.24, para telescópios de neutrinos de com os três volumes abordados. O valor máximo em todos os casos ocorre a uma energia menor, para o neutrino incidente, do que no caso dos taus provenientes do decaimento da NLSP. Além disso, no caso da produção direta de taus o número máximo de regenerações é maior. Isto é esperado dado que não há produção de NLSPs, que podem percorrer grandes distâncias antes de decair. Outro aspecto a ser mencionado, é a variação do número de regenerações em relação à variação do volume do detector, que não muda muito quando comparamos com o caso da detecção indireta de NLSPs.

É importante salientar que os resultados obtidos com esta simulação (produção direta de taus) mostram que nossa simulação da regeneração do tau é consistente com resultados já publicados [28]. Somando-se a isto os resultados reproduzidos para a detecção direta de NLSPs, garantimos maior confiabilidade nos resultados relativos à detecção indireta de NLSPs por meio de seus decaimentos. 

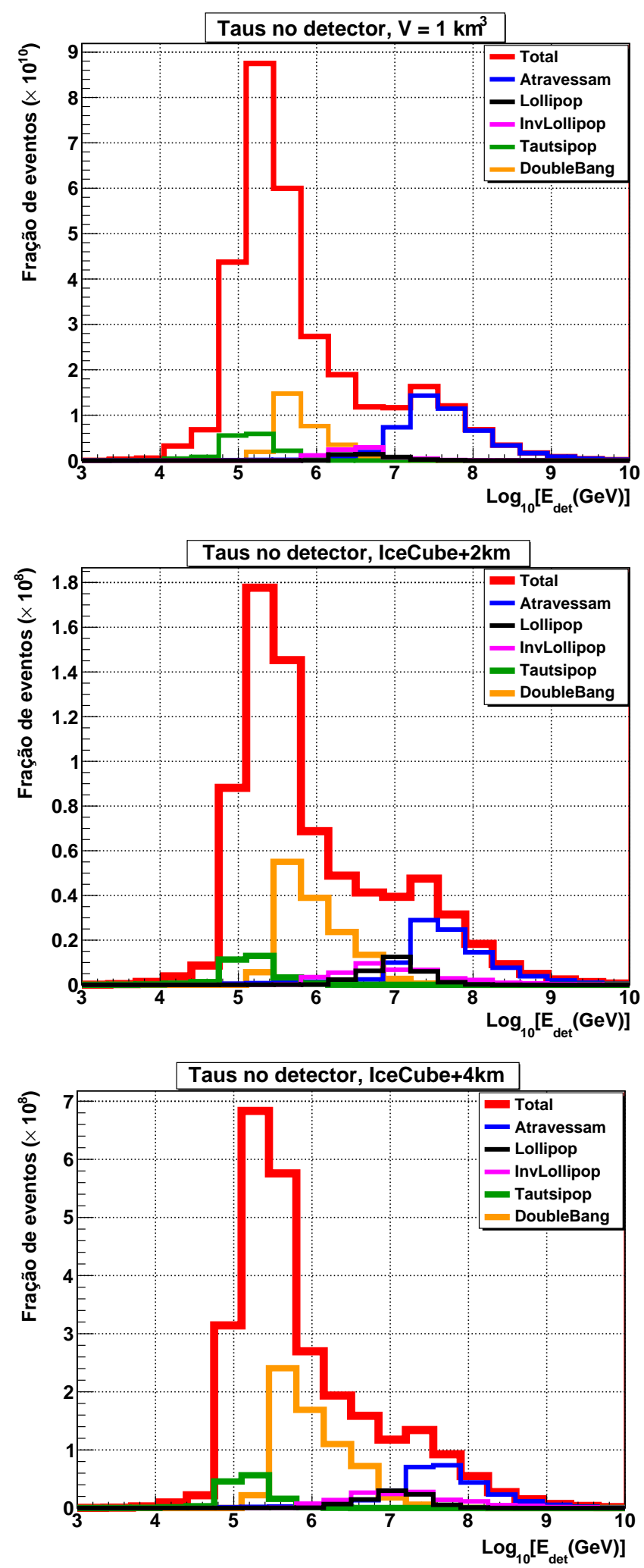

Figura 3.22: Distribuição de energia de taus no IceCube, considerando diferentes assinaturas desta partícula no detector. As normalizações são dadas pelas frações de eventos independentes de fluxo. 

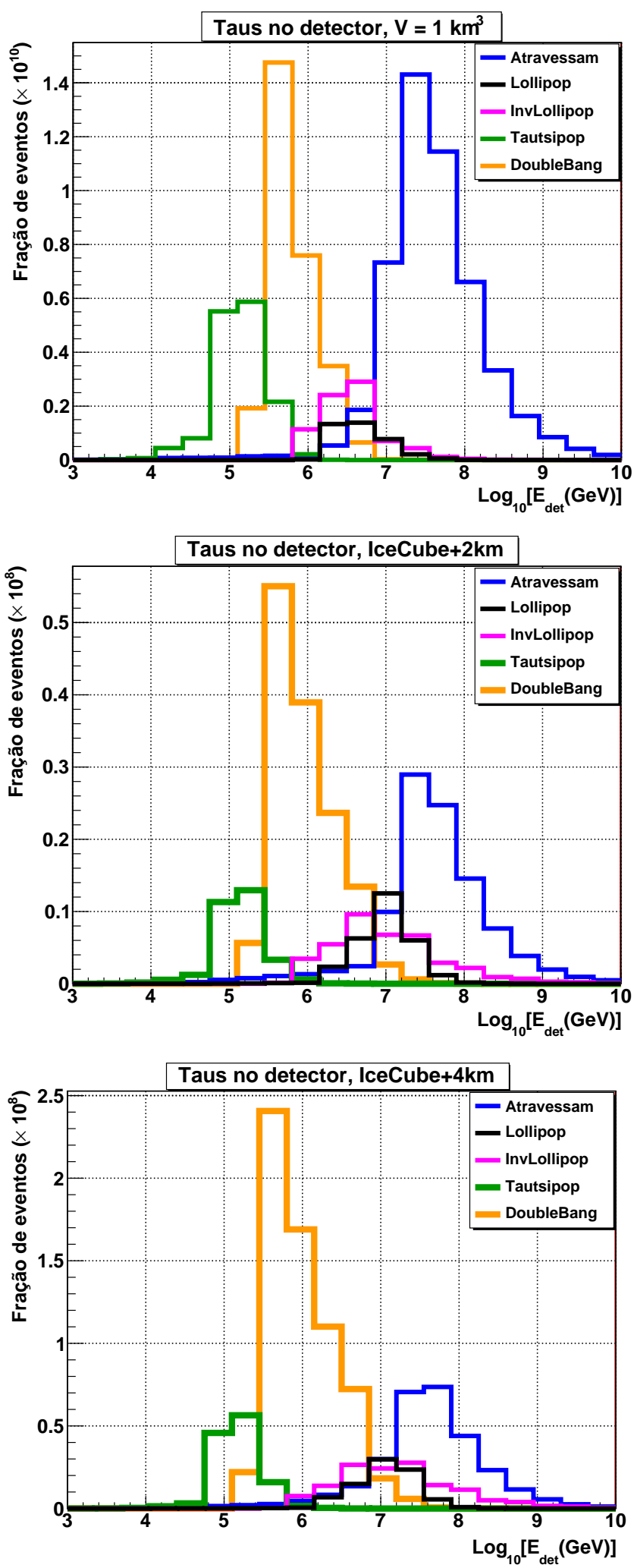

Figura 3.23: Distribuição de energia de taus no IceCube, considerando diferentes assinaturas desta partícula no detector. As normalizações são dadas pelas frações de eventos independentes de fluxo. 


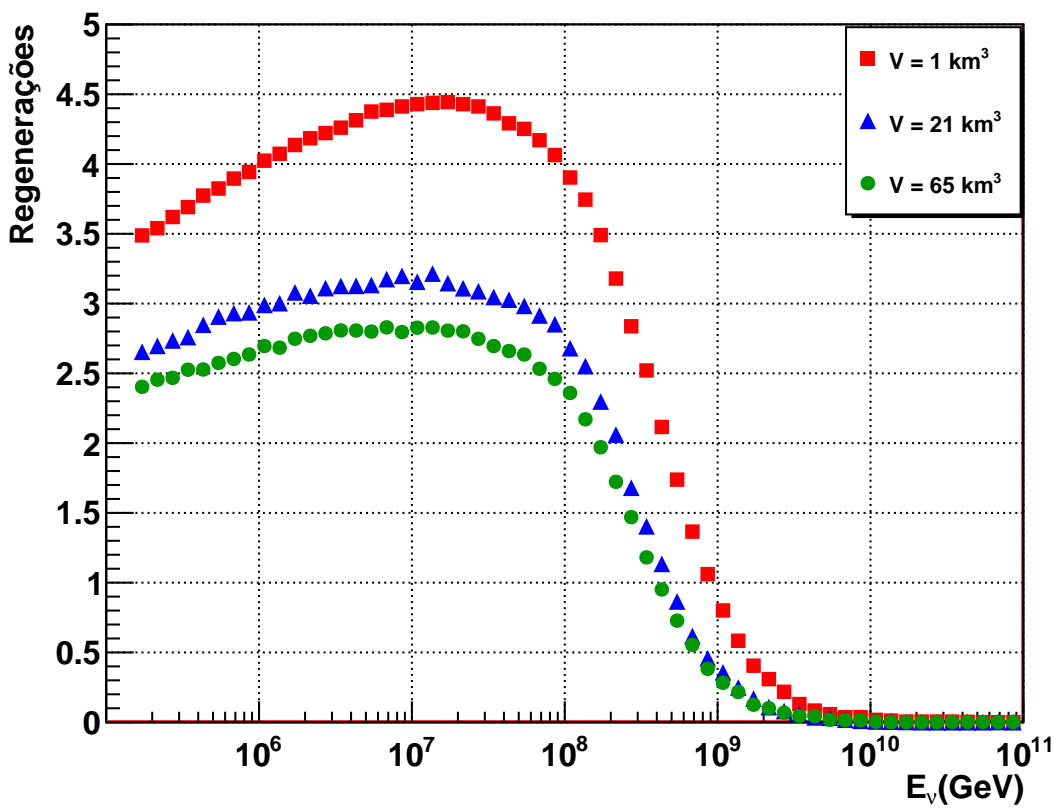

Figura 3.24: Média de regenerações para taus quando não se leva em conta a produção de NLSPs. São considerados 3 possíveis volumes. 


\section{Capítulo 4}

\section{Detecção Indireta de NLSPs - JEM-EUSO}

Como vimos anteriormente, o livre caminho médio do stau depende da escala de quebra de supersimetria $\sqrt{F}$ (equação 2.1). Quanto maior $\sqrt{F}$, maior o tempo de vida média, sendo que para $\sqrt{F}>5 \times 10^{6} \mathrm{GeV}$, uma grande fração das NLSPs atravessam a Terra sem decair. Aqui analisamos os casos em que as NLSPs decaem na atmosfera, após estas partículas saírem do planeta, ou ainda no interior da Terra próximo à superfície de forma que o tau produzido possa emergir com energia suficiente para gerar um chuveiro atmosférico.

Neste caso temos duas possibilidades: 1) sinais provenientes da produção de um chuveiro atmosférico, a partir do decaimento do tau, que ocorre em $82 \%$ de seus decaimentos, com a produção de hádrons e/ou elétrons (tabela 3.1); ou 2) sinais provenientes da interação do (s)tau, que é uma partícula carregada, com a atmosfera. Em ambas as situações o detector usado como referência é o JEM-EUSO [9, 10] (Extreme Universe Space Observatory onboard Japanese Experiment Module), que deve entrar em operação entre 2015 e 2016. Será um telescópio de fluorescência, proposto para se localizar na estação espacial internacional e olhar a Terra a cerca de $400 \mathrm{~km}$ de altitude. Na figura 4.1 é apresentado um esquema de como o JEM-EUSO ficará posicionado em relação à Terra. Vale observar que na nossa análise consideramos partículas que emergem da superfície do planeta em direção à atmosfera, ou seja, no sentido inverso ao do raio cósmico (EECR) mostrado na figura. Isto 
facilita a distinção destes eventos em relação aos chuveiros produzidos por raios cósmicos.

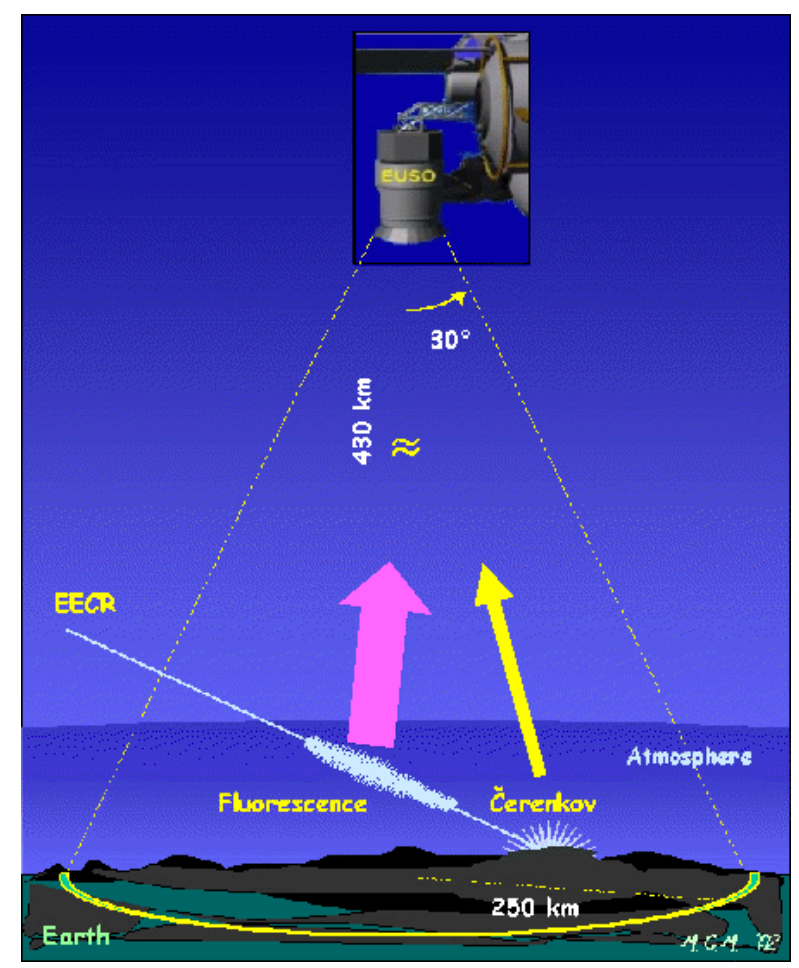

Figura 4.1: Desenho esquemático do experimento JEM-EUSO. Figura obtida de [9].

No caso do telescópio IceCube com volume de $1 \mathrm{~km}^{3}$ o baixo fluxo de taus é devido principalmente às limitações impostas pelo tamanho do volume de detecção e pela vida média do tau, o que restringe as condições nas quais ele pode ser produzido e ser passível de detecção. As condições geométricas do experimento JEM-EUSO superam essas duas restrições. A área de detecção é de cerca de $2 \times 10^{5} \mathrm{~km}^{2}$ e o tau pode ser produzido, e consequentemente decair, em toda a extensão (altitude) da atmosfera, que abrange cerca de $100 \mathrm{~km}$. Isto permite também avaliarmos sinais de coincidências de decaimentos das NLSPs, que são produzidas em pares. A taxa de eventos neste caso será dada pela taxa de NLSPs decaindo em taus dentro do campo de visão do detector. Como o stau é uma partícula carregada, também levamos em conta a possibilidade da geração de fluorescência atmosférica gerada por sua passagem pelo campo de visão do detector, para os casos em que esta partícula não decai. O ponto desfavorável para este experimento é a energia 
mínima aceita pelo detector, cerca de $10^{10} \mathrm{GeV}$, que restringe fortemente a quantidade de eventos passíveis de detecção.

\subsection{Simulação de eventos}

Da mesma forma que no caso da determinação do fluxo de taus no IceCube, temos que levar em conta a geometria do volume de detecção. No caso do JEM-EUSO aproximamos o volume de detecção por um tronco de cone, que forma o campo de visão do detector (figuras 4.1 e 4.2). A superfície superior do tronco de cone assumido é definida pelo limite de altitude adotado para a atmosfera, de $100 \mathrm{~km}$, mais facilmente identificada no esquema da figura 4.1.

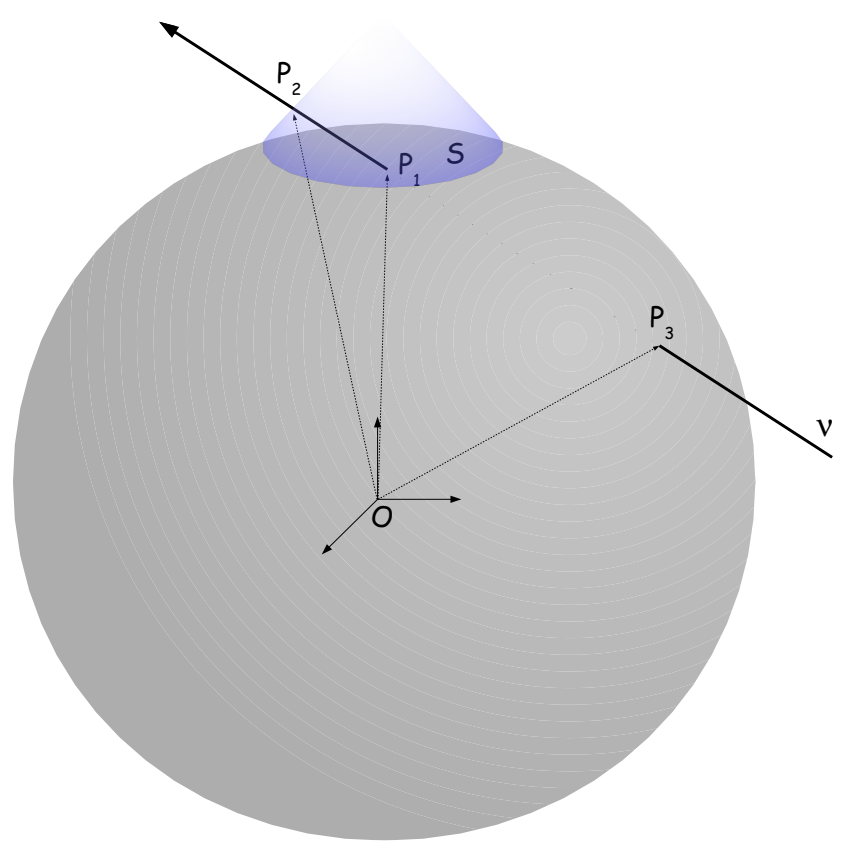

Figura 4.2: Esquema da geometria utilizada para geração das trajetórias das partículas no caso do telescópio JEM-EUSO. $\mathrm{P}_{1}$ está na superfície (S) da Terra e $\mathrm{P}_{2}$ pode estar na superfície lateral ou na superfície superior do tronco de cone definida pelo limite superior da atmosfera. $\mathrm{P}_{3}$ indica o ponto de intersecção da trajetória com a superfície da Terra.

A simulação das trajetórias das partículas é similar ao caso do telescópio 
IceCube. São sorteados dois pontos que definem a trajetória e também o ponto de incidência na superfície da Terra. No caso do JEM-EUSO o ponto $\mathrm{P}_{1}$ é sempre localizado na superfície $\mathrm{S}$ (figura 4.2), enquanto o ponto $\mathrm{P}_{2}$ pode estar na superfície lateral do tronco de cone ou na superfície definida pelo topo da atmosfera. Desta forma são excluídas também as trajetórias acima da horizontal. $\mathrm{P}_{3}$ indica o ponto de intersecção da trajetória definida por $\mathrm{P}_{1}$ e $\mathrm{P}_{2}$ com a Terra, o que define o ponto de entrada do neutrino incidente. Aqui a origem do sistema de coordenadas também é o centro da Terra. Os detalhes dos sorteios das trajetórias são apresentados no apêndice E.

A produção e propagação das partículas foi simulada de acordo com a sequência descrita na seção 3.3, com as diferenças listadas abaixo:

1) O intervalo de energia adotado para os neutrinos incidentes é mais abrangente, tendo seu limite superior em $10^{12} \mathrm{GeV}$, pois o volume de detecção é bem maior;

2) No caso do JEM-EUSO, cada evento equivale a um par de staus, produzidos ao mesmo tempo, como descrito na seção 2.3. Em seguida, determina-se o ponto de decaimento de cada stau do par, independentemente, em relação ao seu ponto de produção. Dessa forma os decaimentos de cada stau do par podem ocorrer em instantes diferentes;

3) É verificado se cada stau do par pode atravessar a Terra, por meio do cálculo do alcance máximo, como descrito na seção 2.5.4. Os eventos em que perdem toda sua energia na trajetória são contados apenas para normalização. Vale observar que praticamente não há perda de energia dos staus quando eles passam pela atmosfera;

4) Verificamos se o ponto de decaimento das partículas que saem da Terra ocorre dentro ou fora do campo de visão do detector. Os que não decaem são adicionados ao grupo de eventos nos quais pode ocorrer geração de fluorescência atmosférica pelo stau. Já os que decaem dentro do campo de visão do JEM-EUSO são adicionados ao grupo de eventos que podem produzir chuveiros atmosféricos proveniente do decaimento do tau. Podemos ainda impor um filtro adicional referente aos decaimentos dos taus, que podem ocorrer fora do campo de visão 
do detector, dependendo da energia com a qual estas partículas são produzidas;

5) São determinadas também as possíveis coincidências temporais entre os staus produzidos em cada par. Elas consistem em eventos para os quais ambos os staus do par decaem dentro do campo de visão do detector. Quando isto ocorre são calculados o intervalo de tempo entre os decaimentos e a distância entre as partículas levando em conta a posição de cada uma no momento do decaimento;

6) O número de eventos por ano no campo de visão do detector, para cada energia, normalizado pelo limite de Waxman e Bahcall é dado pelo fluxo de partículas que atravessam a superfície da Terra (S na figura 4.2) multiplicado pela fração de eventos $F\left(E_{\nu}\right)$ que atingem o campo de visão do detector:

$$
\phi\left(E_{\nu}\right)=F\left(E_{\nu}\right) \times\left(\frac{d \phi_{\nu}}{d E_{\nu}}\right)_{\mathrm{WB}} \times S
$$

O número total de eventos por ano no volume do detector é dado pela integral na energia da equação 4.1 .

\subsection{Fluorescência atmosférica}

Para determinarmos a taxa de eventos na atmosfera, é necessário distinguir eventos que podem gerar sinais mensuráveis em detectores de fluorescência, como o JEM-EUSO. Em princípio temos três tipos de eventos possíveis: i) staus que passam pela atmosfera sem decair; ii) staus que decaem na atmosfera produzindo taus que não decaem no campo de visão do detector; e iii) staus que decaem na atmosfera produzindo taus que decaem dentro do campo de visão do detector. Dessa forma é necessário avaliar a produção de fluorescência atmosférica para estes três casos.

A intensidade de fluorescência atmosférica está relacionada com o número de partículas carregadas $\left(N_{e}\right)$, presentes em cada evento, da seguinte maneira:

$$
\frac{d N}{d x}=N_{f} N_{e}
$$


onde $N_{f}$ é o número de fótons produzidos por partículas carregadas. Geralmente toma-se como referência o número de elétrons e pósitrons, pois são as partículas dominantes em número. Este número é aproximadamente constante $[33,34]$, cerca de 5 fótons por metro por elétron (com energia entre 1 e $1000 \mathrm{MeV})$.

Quando levamos em conta a possibilidade de detecção pelo JEM-EUSO da fluorescência atmosférica gerada pela passagem dos staus que não decaem dentro do campo de visão do detector, o número de partículas carregadas de cada evento se torna um fator limitante. Isto porque neste caso temos $N_{e}=$ 1 ou $N_{e}=2$, visto que a probabilidade destas partículas produzirem um chuveiro atmosférico é desprezível [7]. Sendo assim, estas partículas passam de forma imperceptível ao detector. Este mesmo efeito ocorre nos casos dos taus que não decaem dentro do campo de visão do detector, pois têm pouca chance de interagir com a atmosfera.

Para o terceiro caso mencionado, onde os taus produzidos decaem na atmosfera, a chance de produção de chuveiros atmosféricos é muito grande. Em $82 \%$ dos casos há produção de hádrons e/ou elétrons (tabela 3.1). Desta forma, nos concentramos na determinação das características destes tipos de eventos, pois geram grande quantidade de fluorescência atmosférica. Em especial, os eventos com decaimentos de taus coincidentes no campo de visão do detector podem apresentar características específicas que facilitam sua distinção.

\subsection{Resultados}

Os resultados obtidos nas simulações de decaimentos de NLSPs na atmosfera após atravessarem a Terra são divididos em duas partes: primeiramente são apresentados as taxas de eventos e distribuições de energia e em seguida as distribuições do tempo e de distância entre os decaimentos destas partículas dentro do campo de visão do detector.

\subsubsection{Fluxo na atmosfera e distribuição de energia}

Primeiramente apresentamos os resultados para os casos em que o número de decaimentos de NLSPs em coincidência na atmosfera é máximo. Exemplos 
de espectros de energia obtidos são ilustrados na figura 4.3. São mostradas curvas para todos os staus, para o total de decaimentos de staus e para taus, bem como para os decaimentos em coincidência entre staus e entre taus. Também há curvas para os decaimentos que ocorrem após as partículas passarem pelo campo de visão do detector. A tabela 4.1 mostra o número total de eventos de cada tipo. Notam-se taxas significativas de eventos, tanto para decaimentos em geral quanto para coincidências. Nela não são mostrados os decaimentos de taus porque são muito parecidos com os de staus. Para estes valores de $\sqrt{F}$ o número de taus que não decaem dentro do campo de visão do detector é desprezível.

As distribuições de energia com a qual os staus do par decaem em coincidência na atmosfera, para os mesmos valores de $\sqrt{F}$, são apresentadas na figura 4.4. Elas foram normalizadas pelas taxas apresentadas na tabela 4.1. Os comportamentos são parecidos para as massas do squark de 300 e 600 $\mathrm{GeV}$. Para o caso de $900 \mathrm{GeV}$, a energia fica centrada em um valor mais alto. Isto porque além do limiar para energia de produção de NLSPs ser maior, o valor de $\sqrt{F}$ para massa de $900 \mathrm{GeV}$ é de $2 \times 10^{6} \mathrm{GeV}$, menor do que nos outros dois casos, o que implica em energias mais altas para NLSPs decaírem na atmosfera (figura 3.2). Como pode ser notado, estas distribuições de energia apresentam valores médios muito abaixo da energia mínima aceita pelo JEM-EUSO $\left(\sim 10^{10} \mathrm{GeV}\right)$.

Na figura 4.5 são apresentadas curvas do número total de decaimentos de NLSPs em coincidência na atmosfera em função de $\sqrt{F}$. Foi tomado um espectro de valores entre $7 \times 10^{4}$ e $1,3 \times 10^{7} \mathrm{GeV}$. Nota-se que a sensibilidade do número de coincidências é bastante sensível à variação de $\sqrt{F}$, com os valores máximos apresentados também na tabela 4.1. Para cada ponto dos gráficos da figura 4.5 temos também o valor médio da energia destas partículas ao decaírem dentro do campo de visão do detector, na figura 4.6, onde as incertezas de cada ponto representam o desvio-padrão da distribuição correspondente. Neste caso é observado um aumento sistemático no valor da energia das NLSPs com a diminuição do valor de $\sqrt{F}$, pois apenas as mais energéticas conseguem percorrer distâncias suficientemente grandes a ponto de decaírem após atravessarem a Terra. 

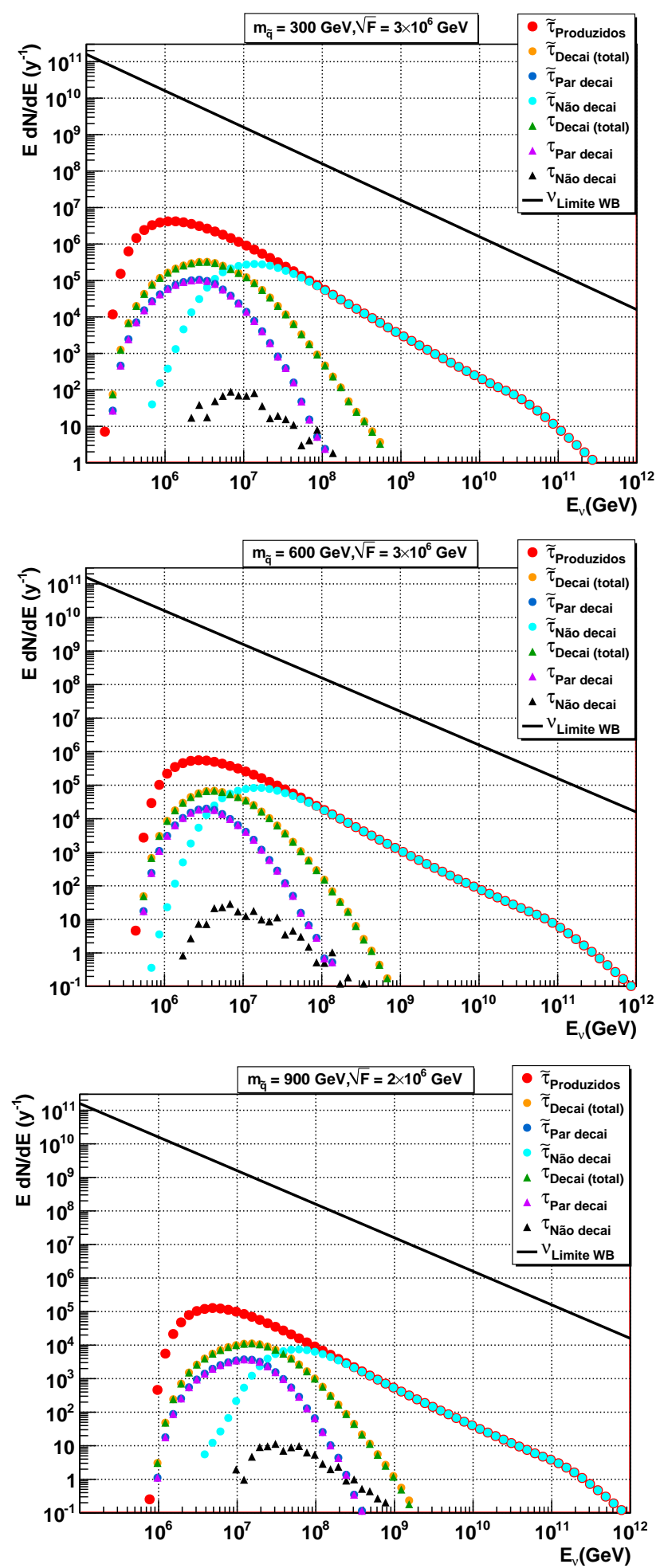

Figura 4.3: Eventos por ano em função da energia do neutrino incidente, tendo como referência o campo de visão do telescópio JEM-EUSO. Em vermelho temos todos os staus; em laranja e verde, os decaimentos de staus e taus que ocorrem dentro do campo de visão do detector; círculos em ciano e triângulos pretos, decaimentos de staus e taus que ocorrem após o campo de visão do detector; em azul e roxo, decaimentos de NLSPs e de taus em coincidência dentro do campo de visão do detector. Também é apresentado o limite de Waxman e Bahcall. 
Tabela 4.1: Fluxo máximo de NLSPs por ano na atmosfera para diferentes situações de decaimento, com $\sqrt{F}=3 \times 10^{6} \mathrm{GeV}$ para $m_{\tilde{q}}$ de 300 e $600 \mathrm{GeV}$ e com $\sqrt{F}=2 \times 10^{6}$ para $m_{\tilde{q}}=900 \mathrm{GeV}$. "Total" corresponde a todos os staus produzidos; "Não decaem", aos decaimentos que ocorrem fora do campo de visão do detector; "Decaem (total)", ao total de decaimentos dentro do campo de visão do detector; e "Par decai", aos decaimentos em coincidência dos staus do par dentro do campo de visão do detector com geração de um par de taus.

\begin{tabular}{c|c|c|c|c}
\hline$m_{\tilde{q}}$ & Total & Não decaem & Decaem (total) & Par decai \\
\hline 300 & $1 \times 10^{7}$ & $7,2 \times 10^{5}$ & $7,4 \times 10^{5}$ & $2,2 \times 10^{5}$ \\
\hline 600 & $1,3 \times 10^{6}$ & $2,0 \times 10^{5}$ & $1,3 \times 10^{5}$ & $3,0 \times 10^{4}$ \\
\hline 900 & $2,8 \times 10^{5}$ & $1,7 \times 10^{4}$ & $2,4 \times 10^{4}$ & $7,0 \times 10^{3}$ \\
\hline
\end{tabular}

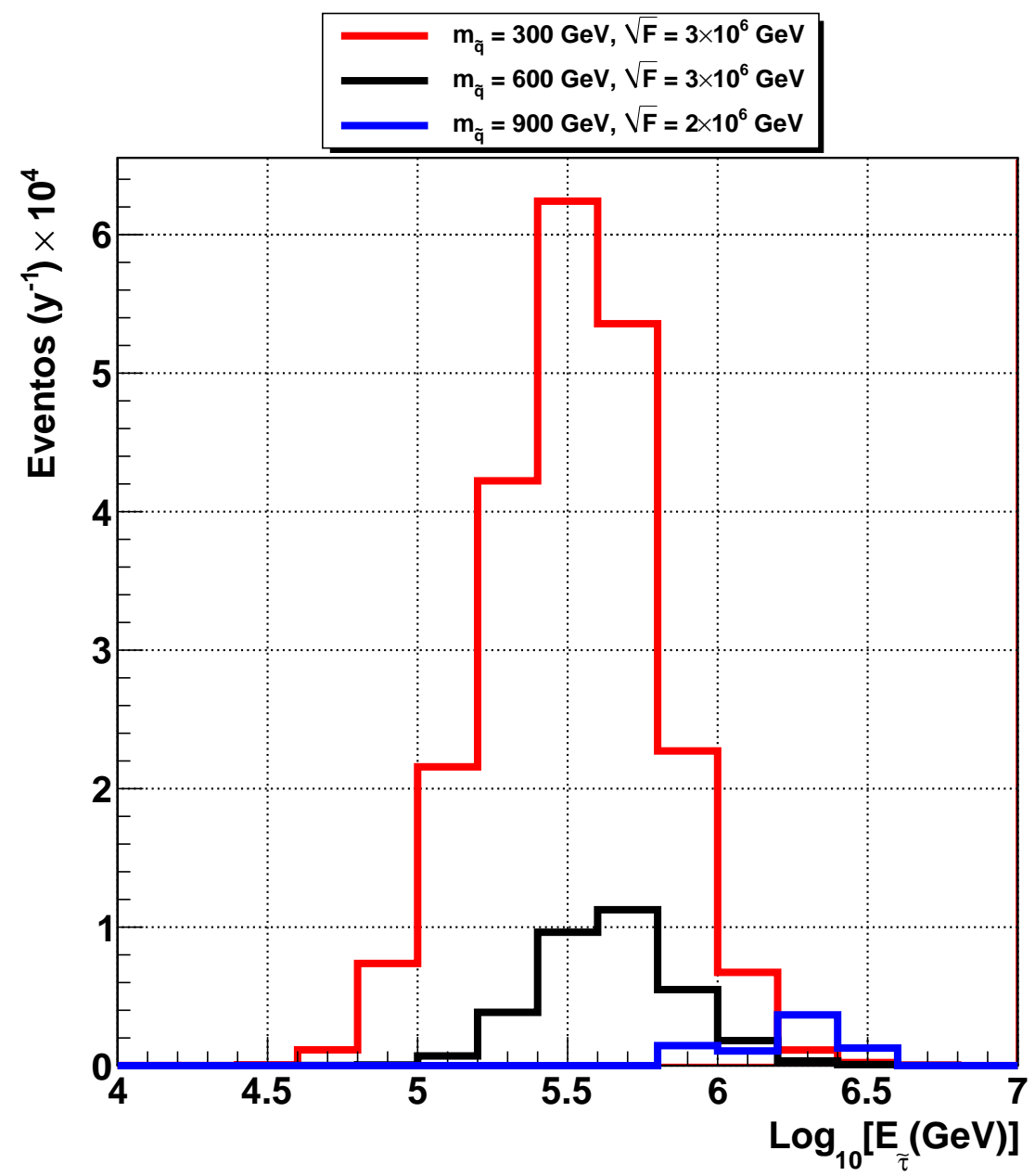

Figura 4.4: Distribuição de energia dos staus que decaem em coincidência na atmosfera. 


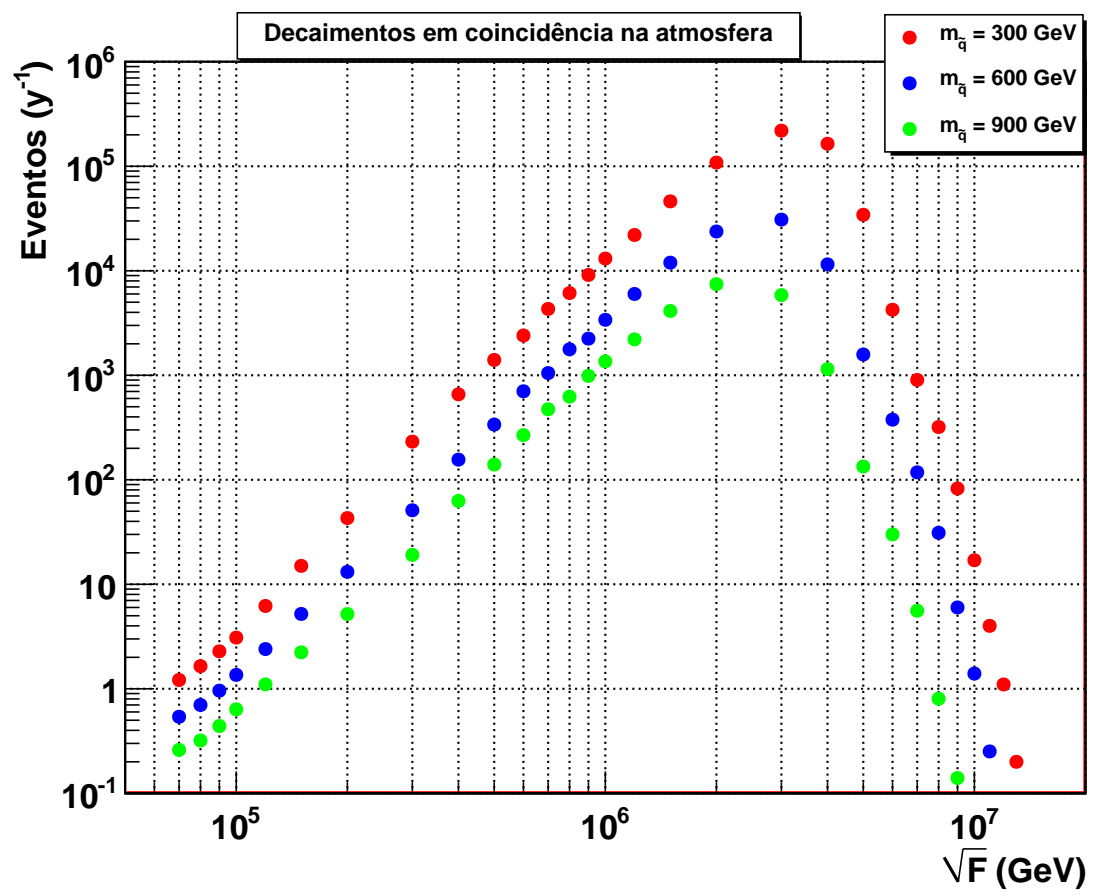

Figura 4.5: Coincidências de decaimentos de staus por ano, dentro do campo de visão do JEM-EUSO, em função de $\sqrt{F}$.

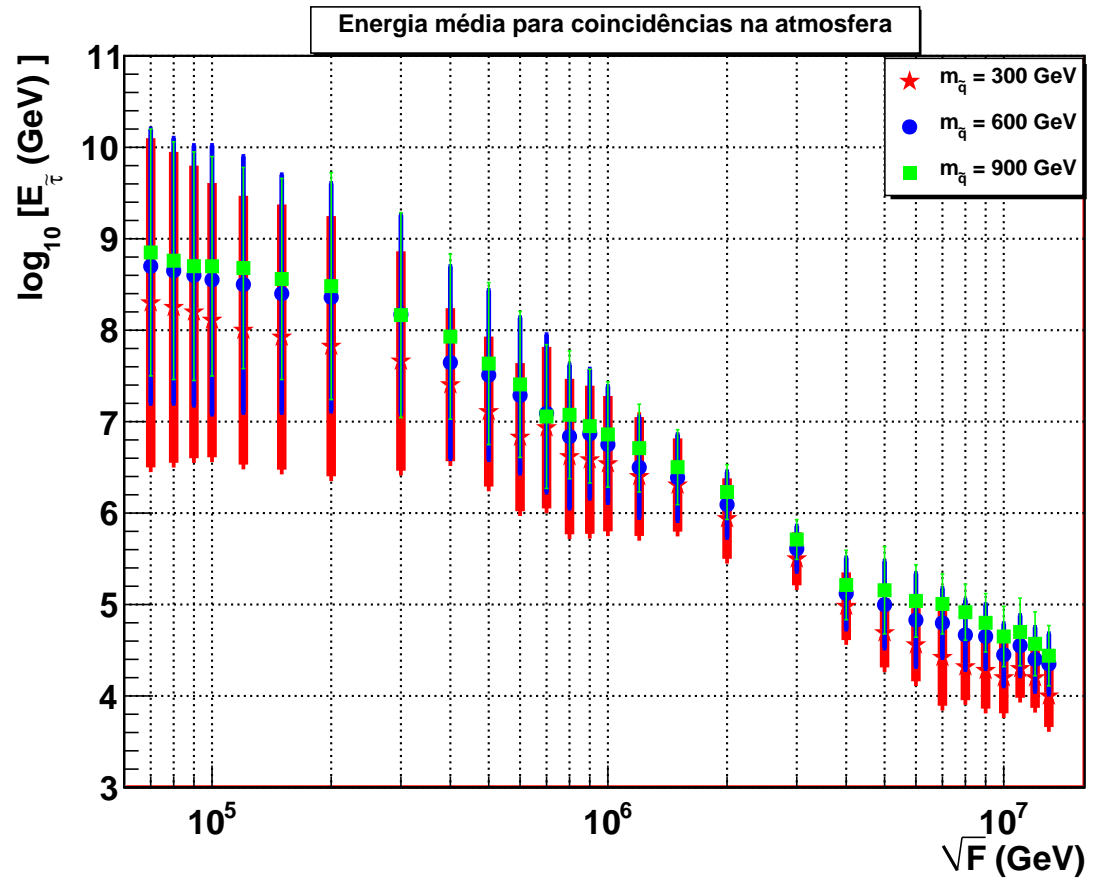

Figura 4.6: Energia média dos staus que decaem em coincidências dentro do campo de visão do JEM-EUSO, em função de $\sqrt{F}$. As incertezas dos pontos representam os desvios-padrão das distribuições. 
Nossos resultados indicam que o número de eventos pode ser bastante elevado dependendo do valor de $\sqrt{F}$, chegando a cerca de $2 \times 10^{5}$ no melhor cenário. Entretanto, neste caso, a energia média observada nos decaimentos é muito baixa quando comparada com a energia mínima aceita pelo JEMEUSO. Por outro lado, nota-se que para valores menores de $\sqrt{F}$ a energia média das partículas é maior. Neste sentido realizamos novos mapeamentos do número de eventos em função de $\sqrt{F}$ impondo restrições para a energia das NLSPs ao decaírem na atmosfera. Determinamos as taxas de eventos para os decaimentos na atmosfera produzidos por staus cujas energias são maiores do que 3 valores específicos: $10^{8}, 10^{9}$ e $10^{10} \mathrm{GeV}$. Incluímos também valores de energia média abaixo do mínimo aceito pelo JEM-EUSO com o intuito de incentivar novos experimentos que porventura possam se enquadrar nestas condições (figura 4.7).

Nota-se nesta figura que os valores de $\sqrt{F}$ correspondentes ao máximo de eventos são diferentes para cada corte de energia, diminuindo sistematicamente à medida em que aumentamos o valor mínimo da energia com a qual as NLSPs decaem na atmosfera, em acordo com a figura 4.6. Para cada caso há uma pequena mudança também em relação à massa do squark. Por exemplo, quando $E_{\tilde{\tau}} \geq 10^{8}$ temos o máximo de eventos em $7 \times 10^{5} \mathrm{GeV}$ para $m_{\tilde{q}}=300 \mathrm{GeV}$ e $6 \times 10^{5} \mathrm{GeV}$ para $m_{\tilde{q}}=600$ e $900 \mathrm{GeV}$. Este efeito está relacionado com a energia mínima necessária para produzir as NLSPs em função da massa do squark.

Na tabela 4.2 são apresentados os números máximos de eventos por ano para as restrições de energia, aos decaimentos dentro do campo de visão do detector, impostas anteriormente. Nela também são reapresentados os resultados da tabela 4.1 a título de comparação. Temos, portanto, números significativos de decaimentos de NLSPs em coincidência até mesmo para energias muito elevadas. O número de decaimentos de taus só é mais limitado quando $E_{\tilde{\tau}} \geq 10^{10} \mathrm{GeV}$. 

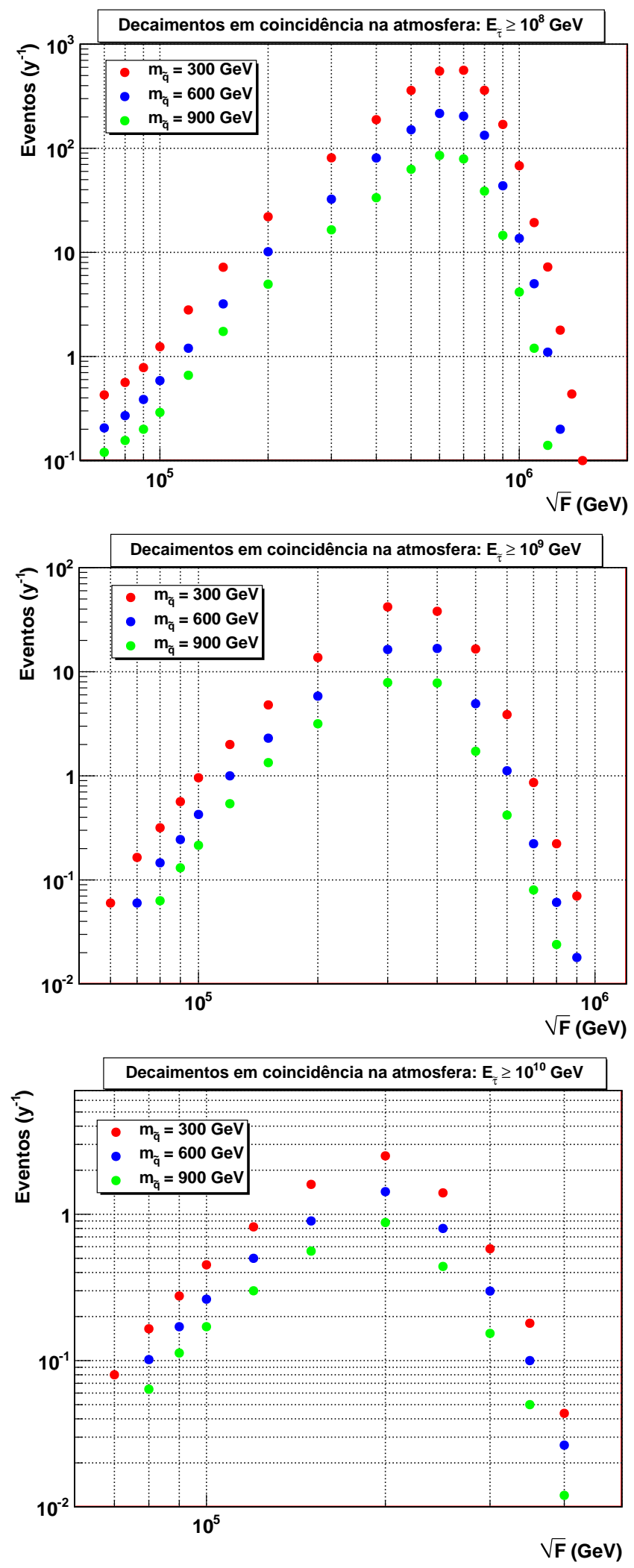

Figura 4.7: Coincidências de decaimentos de staus por ano, dentro do campo de visão do JEM-EUSO, em função de $\sqrt{F}$, quando $E_{\tilde{\tau}} \geq 10^{8}, 10^{9}, 10^{10} \mathrm{GeV}$. 
Tabela 4.2: Eventos por ano quando são impostas as restrições de energia. Também são apresentados as taxas de decaimentos dos taus. Para $\sqrt{F}=7 \times 10^{5}$ $\mathrm{GeV}, E_{\tilde{\tau}} \geq 10^{8} \mathrm{GeV}$; para $\sqrt{F}=3 \times 10^{5} \mathrm{GeV}, E_{\tilde{\tau}} \geq 10^{9} \mathrm{GeV}$; e para $\sqrt{F}=2 \times 10^{5}$ $\mathrm{GeV}, E_{\tilde{\tau}} \geq 10^{10} \mathrm{GeV}$.

\begin{tabular}{|c|c|c|c|c|c|}
\hline \multicolumn{6}{|c|}{$m_{\tilde{q}}=300 \mathrm{GeV}$} \\
\hline$\sqrt{F}(\mathrm{GeV})$ & $E_{\tilde{\tau}}(\mathrm{GeV})$ & $\tilde{\tau}_{\text {Decai }(\text { total })}$ & $\tilde{\tau}_{\text {Coincidentes }}$ & $\tau_{\text {Decai }(\text { total })}$ & $\tau_{\text {Coincidentes }}$ \\
\hline $3 \times 10^{6}$ & todas & $7,4 \times 10^{5}$ & $2,2 \times 10^{5}$ & $7,2 \times 10^{5}$ & $2,0 \times 10^{5}$ \\
\hline $7 \times 10^{5}$ & $\geq 10^{8}$ & $1,6 \times 10^{3}$ & $5,0 \times 10^{2}$ & $1,7 \times 10^{3}$ & $5,5 \times 10^{2}$ \\
\hline $3 \times 10^{5}$ & $\geq 10^{9}$ & $1,1 \times 10^{2}$ & $4,0 \times 10^{1}$ & $1,3 \times 10^{2}$ & $3,4 \times 10^{1}$ \\
\hline $2 \times 10^{5}$ & $\geq 10^{10}$ & 6,4 & 2,5 & 1,6 & $1,6 \times 10^{-1}$ \\
\hline \hline \multicolumn{6}{|c|}{$m_{\tilde{q}}=600 \mathrm{GeV}$} \\
\hline$\sqrt{F}(\mathrm{GeV})$ & $E_{\tilde{\tau}}(\mathrm{GeV})$ & $\tilde{\tau}_{\text {Decai }(\text { total })}$ & $\tilde{\tau}_{\text {Coincidentes }}$ & $\tau_{\text {Decai }(\text { total })}$ & $\tau_{\text {Coincidentes }}$ \\
\hline $3 \times 10^{6}$ & todas & $1,3 \times 10^{5}$ & $3,0 \times 10^{4}$ & $1,3 \times 10^{5}$ & $3,0 \times 10^{4}$ \\
\hline $6 \times 10^{5}$ & $\geq 10^{8}$ & $6,4 \times 10^{2}$ & $2,2 \times 10^{2}$ & $5,8 \times 10^{2}$ & $2,0 \times 10^{2}$ \\
\hline $4 \times 10^{5}$ & $\geq 10^{9}$ & $5,3 \times 10^{1}$ & $1,7 \times 10^{1}$ & $3,7 \times 10^{1}$ & 9,5 \\
\hline $2 \times 10^{5}$ & $\geq 10^{10}$ & 3,4 & 1,4 & 1,1 & $1,1 \times 10^{-1}$ \\
\hline \hline \multicolumn{6}{|c|}{$m_{\tilde{q}}=900 \mathrm{GeV}$} \\
\hline$\sqrt{F}(\mathrm{GeV})^{2}$ & $E_{\tilde{\tau}}(\mathrm{GeV})$ & $\tilde{\tau}_{\text {Decai (total })}$ & $\tilde{\tau}_{\text {Coincidentes }}$ & $\tau_{\text {Decai(total })}$ & $\tau_{\text {Coincidentes }}$ \\
\hline $2 \times 10^{6}$ & todas & $2,4 \times 10^{4}$ & $7,0 \times 10^{3}$ & $2,4 \times 10^{4}$ & $7,0 \times 10^{3}$ \\
\hline $6 \times 10^{5}$ & $\geq 10^{8}$ & $2,6 \times 10^{2}$ & $8,5 \times 10^{1}$ & $2,7 \times 10^{2}$ & $9,5 \times 10^{1}$ \\
\hline $3 \times 10^{5}$ & $\geq 10^{9}$ & $2,2 \times 10^{1}$ & 7,9 & $2,3 \times 10^{1}$ & 6,0 \\
\hline $2 \times 10^{5}$ & $\geq 10^{10}$ & 2,4 & $8,8 \times 10^{-1}$ & $6,0 \times 10^{-1}$ & $5,3 \times 10^{-2}$ \\
\hline
\end{tabular}

\subsubsection{Distância e tempo entre os decaimentos}

Na figura 4.8 são apresentadas distribuições normalizadas das diferenças de tempo entre os instantes de decaimento dos pares de staus quando ambos decaem dentro do campo de visão do JEM-EUSO. Para cada massa do squark produzido na interação neutrino-núcleon são mostradas quatro situações de coincidências de decaimentos: para os valores de $\sqrt{F}$ que promovem o máximo de coincidência na atmosfera sem restrição de energia dos staus e com restrições de energias destas partículas decaindo na atmosfera acima de $10^{8}, 10^{9}$ e $10^{10} \mathrm{GeV}$. Notamos que para todos os casos as distribuições ficam em torno de $10^{-3,7}$ segundos. Dado que o tempo de duração de um chuveiro atmosférico é da ordem de 0,3 segundos (tempo aproximado para a luz atravessar a atmosfera) e que a resolução temporal do detector é de $2,5 \mu s$, é possível identificar que ambos os decaimentos ocorrem em coin- 
cidência. Similarmente, na figura 4.9 mostramos as distribuições da distância entre os decaimentos dos pares de staus na atmosfera, também com normalização arbitrária. As distribuições têm forma de exponencial, com valor mais provável entre aproximadamente 50 e 100 km. Dado que a resolução espacial do detector é $0,75 \mathrm{~km}$, é possível identificar espacialmente os dois chuveiros produzidos nos decaimentos das NLSPs.
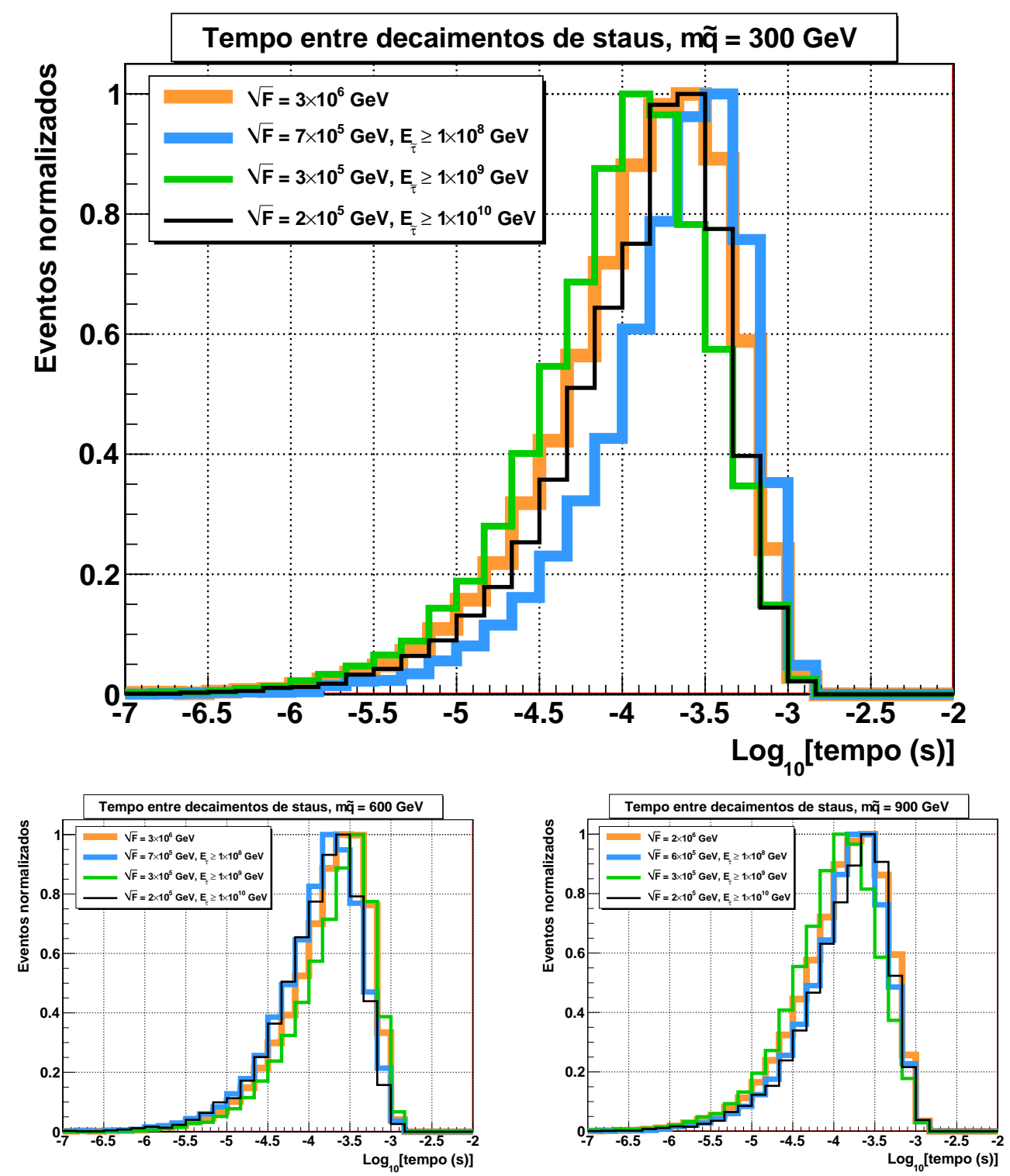

Figura 4.8: Distribuições diferença de tempo entre os instantes de decaimentos dos pares de staus dentro do campo de visão do JEM-EUSO, para diferentes valores de $\sqrt{F}$ e restrições de energia mínima destas partículas. 

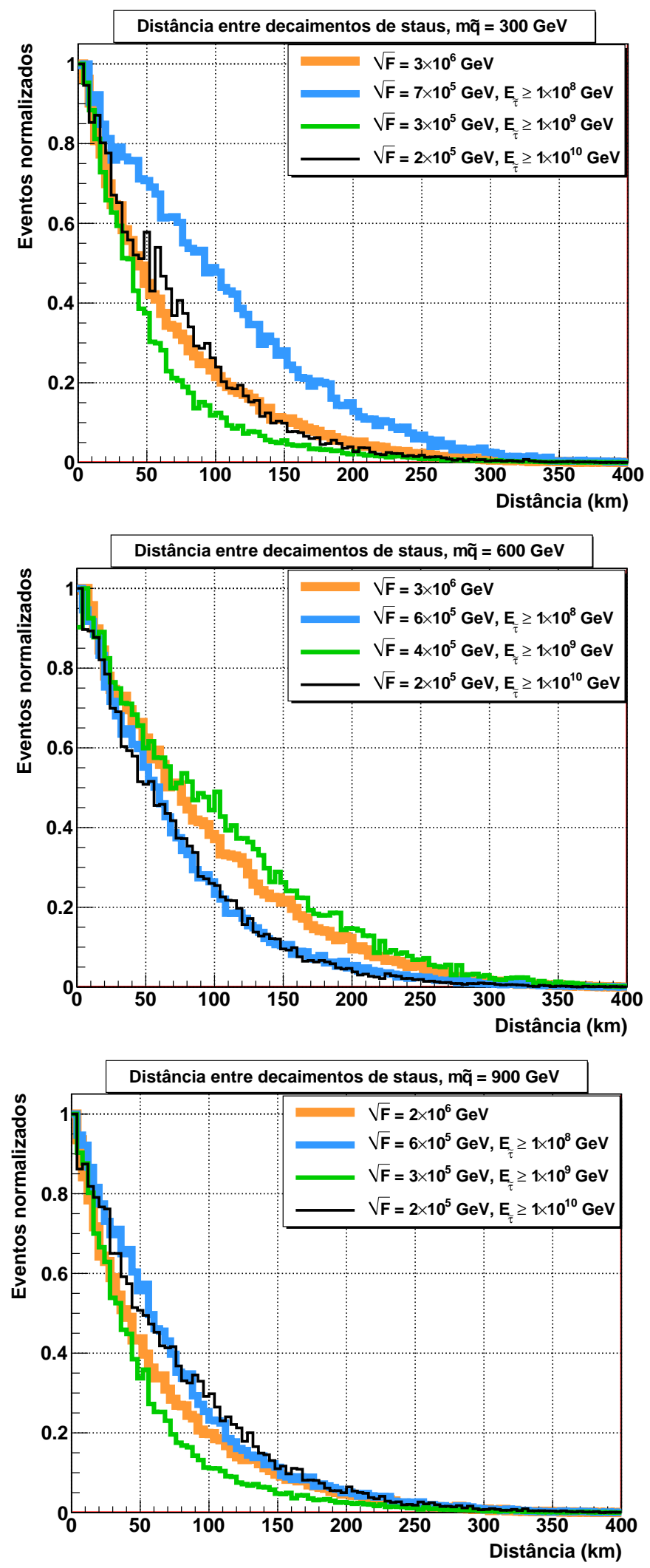

Figura 4.9: Distribuições da distância entre os pontos de decaimento dos pares de staus quando estes decaimentos ocorrem dentro do campo de visão do detector, para diferentes valores de $\sqrt{F}$ e restrições de energia para estas partículas. 


\section{Capítulo 5}

\section{Discussão}

Como primeiro resultado deste trabalho temos a determinação da taxa de taus e múons, originados do decaimentos de NLSPs, que atingem telescópios de neutrinos de $1 \mathrm{~km}^{3}$ de volume, como o IceCube, bem como telescópios similares com volumes maiores. O melhor cenário para a taxa de taus é obtido quando adotamos valores para $\sqrt{F} \lesssim 2 \times 10^{6} \mathrm{GeV}$, como mostrado na figura 3.9. Nestas condições, a taxa máxima de taus que chegam ao telescópio IceCube em condições de serem detectados é de cerca 0,07 eventos por ano (tabela 3.2). Para o caso de múons provenientes do decaimento do tau a taxa máxima obtida é de 0,2 eventos por ano. Quando levamos em conta um aumento no raio do IceCube em 2 e $4 \mathrm{~km}$, obtemos um aumento significativo no fluxo de taus e também dos múons provenientes de seu decaimento, onde a taxa máxima é de 10 eventos por ano (tabela 3.3). Contudo, para avaliarmos de forma mais realista a viabilidade de se observar experimentalmente esses sinais de taus e múons de decaimentos de NLSPs em telescópios de neutrinos, é necessário levar em conta os principais eventos de fundo e as principais limitações experimentais.

Os taus devem proporcionar assinaturas muito específicas no detector [27], praticamente livres de fundo. Entretanto é necessário levar em consideração que apenas uma parte dos taus tem maior chance de deixar assinaturas bem definidas no IceCube: cerca de $18 \%$ para $1 \mathrm{~km}^{3} ; 32 \%$ para $21 \mathrm{~km}^{3}$ e de $37 \%$ para $65 \mathrm{~km}^{3}$, como pode ser observado na tabela 3.4. Comparando as 
distribuições de energia apresentadas na figura 3.7 com as das figuras $3.22 \mathrm{e}$ 3.23 (para $1 \mathrm{~km}^{3}$ ), notamos que uma parte significativa dos taus provenientes de NLSPs chegam com energia suficiente para atravessar o detector. Estes são, em princípio, muito difíceis de distinguir de outros sinais [36], como de múons provenientes de neutrinos atmosféricos, por exemplo. Entretanto esta possibilidade não pode ser descartada. Nestas condições teríamos cerca de $1,5 \times 10^{-2}$ sinais de taus por ano em condições de detecção para $1 \mathrm{~km}^{3}$, no melhor cenário. Já para os volumes maiores seriam 0,5 eventos por ano no caso de $21 \mathrm{~km}^{3}$ e 1,6 para $65 \mathrm{~km}^{3}$.

Os múons de decaimentos do tau tem como principal fundo, múons provenientes de interações de neutrinos atmosféricos. Para tentar estimar a taxa de eventos de neutrinos atmosféricos na escala de energia que estamos trabalhando, foi realizada uma extrapolação do fluxo de neutrinos atmosféricos medido no telescópio IceCube [35]. A curva obtida nesta referência é determinada para energias do neutrino incidente de até $400 \mathrm{TeV}$. Por isto é importante salientar que a extrapolação adotada aqui é conservadora, pois assume que o comportamento da queda do fluxo dos eventos de neutrinos atmosféricos se mantém para energias acima desse valor. Entretanto o fluxo de raios cósmicos é proporcional a $E^{-2,7}$ até cerca de $10^{6} \mathrm{GeV}$, tendo sua redução intensificada para $E^{-3}$ entre este valor e cerca de $5 \times 10^{8} \mathrm{GeV}$, avançando, então, para $E^{-3,2}$. Ele só passa a ser proporcional a $E^{-2,7}$ novamente para partículas de energias acima de $\sim 3 \times 10^{9} \mathrm{GeV}$.

Na figura 5.1 apresentamos no primeiro gráfico curvas da soma dos fluxos de taus e múons do decaimento de NLSPs para as três massas do squark, onde é possível visualizar também o efeito da variação da massa do squark, cuja principal causa é a dependência da seção de choque em relação a este parâmetro (figura 2.4). No segundo gráfico temos curvas do tau, múon e da soma de ambos para apenas um valor de massa do squark, onde é possível identificar a contribuição da taxa de cada uma à soma. Como a extrapolação do fluxo de eventos de neutrinos atmosféricos é superestimada, apresentamos duas estimativas mostradas em ambos os gráficos: a linha contínua é obtida da extrapolação explicada acima e a pontilhada corresponde a um deslocamento arbitrário da primeira curva para a esquerda. Nota-se que a soma das taxas de taus com as de múons só é comparável ao fluxo representado pela linha contínua para energias de incidência por volta de $10^{8} \mathrm{GeV}$. Ou seja, 
para energias abaixo deste valor o fluxo de múons provenientes do decaimento das NLSPs estaria dentro da flutuação estatística dos eventos de neutrinos atmosféricos. A curva pontilhada é obtida de tal forma que ela coincida com a soma máxima das taxas de taus e múons de decaimentos de NLSPs para uma energia de incidência de $10^{7} \mathrm{GeV}$.

Determinamos, então, o fluxo de taus e múons para energias de incidência acima de $10^{7}$ e $10^{8} \mathrm{GeV}$, cujos resultados são apresentados na tabela 5.1. Nota-se que, para o primeiro caso, o número de múons sofre uma redução bastante significativa, para cerca de $1 / 4$ do total, implicando em cerca de 1 evento a cada dez anos. Para o corte em $10^{8} \mathrm{GeV}$, teríamos uma quantidade de múons irrisória. A taxa total de múons de neutrinos atmosféricos é de 0,006 eventos por ano para energias do neutrino acima de $10^{8}$ para a curva contínua ou acima de $10^{7}$ para a curva pontilhada. Isto significa que para o caso menos conservador, os eventos obtidos na tabela são praticamente livres de fundo. Desta forma poderíamos ter cerca de 0,07 eventos por ano, somando-se estes eventos aos taus com maiores chances de serem identificados. Ao compararmos estes resultados com a previsão para a detecção de matéria escura no IceCube, um dos objetivos científicos deste experimento, vemos que é um número relativamente baixo, pois temos menos de um evento a cada 10 anos. Para este experimento as previsões para a detecção de matéria escura exigem cerca de dez anos de medidas para atingir estatística suficiente, atentando-se ao fato de serem previsões altamente dependentes de modelos.

Para o caso do IceCube ampliado temos os resultados apresentados na tabela 5.2, obtidos para taus e múons supondo um comportamento similar ao caso de $1 \mathrm{~km}^{3}$, onde o número de taus mais múons provenientes do decaimento de NLSPs é comparável ao número de eventos de neutrinos atmosféricos para partículas incidentes de energias acima de $10^{7}$ e $\sim 10^{8} \mathrm{GeV}$. Nota-se que as taxas obtidas continuam sendo significativas, mesmo com a restrição mais conservadora. Teríamos nos melhores cenários, entre 1 e 2 sinais de múons por ano com energias acima do esperado para sinais de neutrinos atmosféricos. 

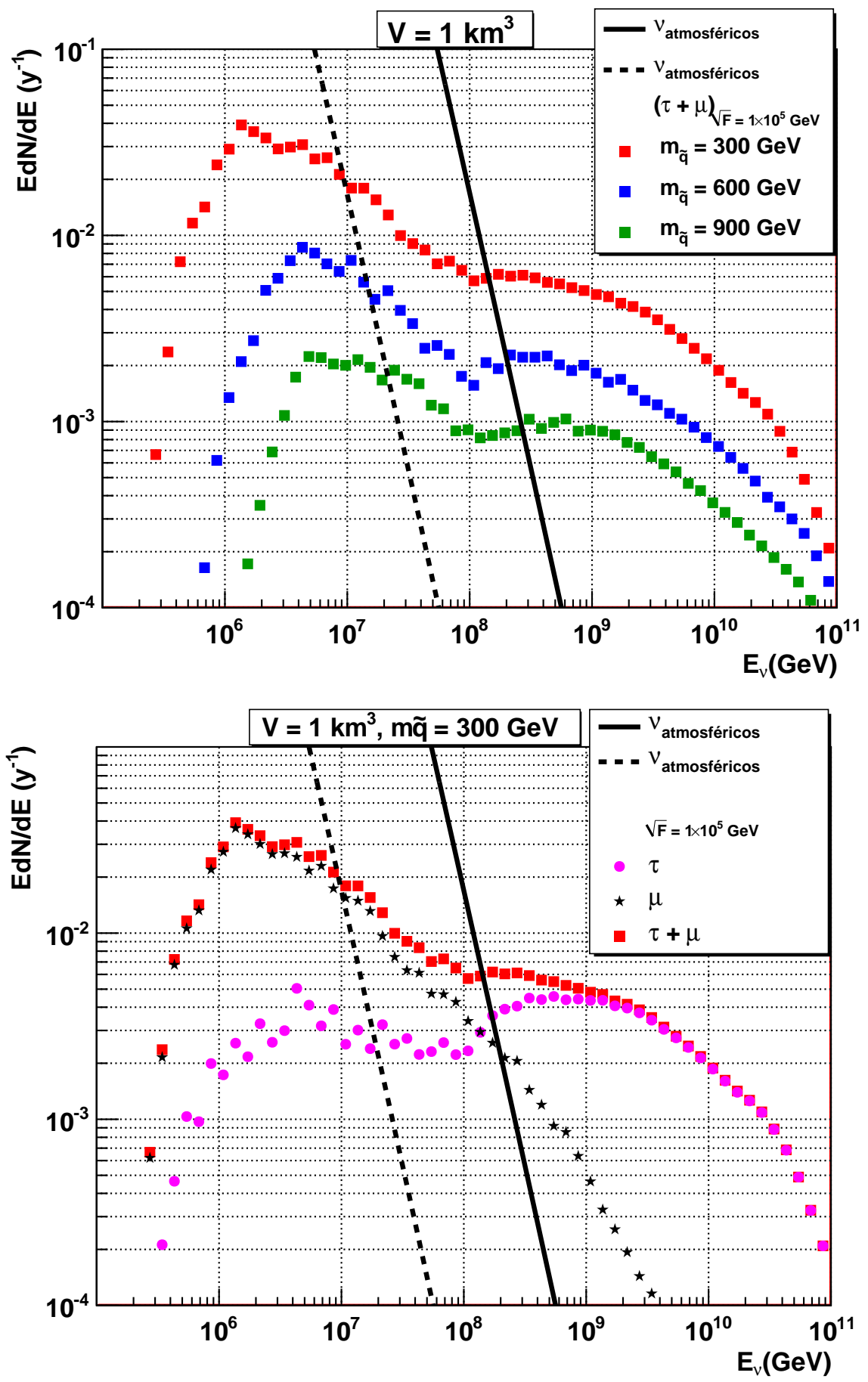

Figura 5.1: Comparação entre os fluxos de staus e a soma dos fluxos de taus e múons, por ano num telescópio de neutrinos de $1 \mathrm{~km}^{3}$, como o IceCube. Para os taus e múons (provenientes do decaimento do tau), $\sqrt{F}=1 \times 10^{5} \mathrm{GeV}$. No caso dos staus, $\sqrt{F}=5 \times 10^{7} \mathrm{GeV}$. É apresentado também um fluxo extrapolado de neutrinos atmosféricos. 
Tabela 5.1: Taxas de taus e múons, resultantes do decaimento de NLSPs, por ano em um telescópio de neutrinos de $1 \mathrm{~km}^{3}$ situado a cerca de $2 \mathrm{~km}$ de profundidade, com $\sqrt{F}=1 \times 10^{5} \mathrm{GeV}$. Foram adotados dois valores de energia mínima do neutrino incidente: $10^{7}$ e $10^{8} \mathrm{GeV}$.

\begin{tabular}{|c|c|c|c|}
\hline \multicolumn{4}{|c|}{$E_{\nu}>10^{7} \mathrm{GeV}$} \\
\hline $\mathrm{m}_{\tilde{q}}(\mathrm{GeV})$ & $\tau\left(\mathrm{y}^{-1}\right)$ & $\mu\left(\mathrm{y}^{-1}\right)$ & $\mu+\tau\left(\mathrm{y}^{-1}\right)$ \\
\hline 300 & 0,051 & 0,053 & 0,10 \\
600 & 0,020 & 0,017 & 0,04 \\
900 & 0,009 & 0,007 & 0,02 \\
\hline \multicolumn{4}{|c|}{$E_{\nu}>10^{8} \mathrm{GeV}$} \\
\hline $\mathrm{m}_{\tilde{q}}(\mathrm{GeV})$ & $\tau\left(\mathrm{y}^{-1}\right)$ & $\mu\left(\mathrm{y}^{-1}\right)$ & $\mu+\tau\left(\mathrm{y}^{-1}\right)$ \\
\hline 300 & 0,038 & 0,010 & 0,048 \\
600 & 0,015 & 0,003 & 0,018 \\
900 & 0,007 & 0,002 & 0,009 \\
\hline
\end{tabular}

Tabela 5.2: Eventos de taus e múons por ano nos volumes especificados, para $E_{\nu}$ $>10^{8} \mathrm{GeV}$, massas do squark de 300,600 e $900 \mathrm{GeV}$ e $\sqrt{F}=1 \times 10^{5} \mathrm{GeV}$.

\begin{tabular}{|c|c|c|c|c|}
\hline \multicolumn{5}{|c|}{$E_{\nu}>10^{7} \mathrm{GeV}$} \\
\hline $\mathrm{m}_{\tilde{q}}(\mathrm{GeV})$ & $\mathrm{V}\left(\mathrm{km}^{3}\right)$ & $\tau\left(\mathrm{y}^{-1}\right)$ & $\mu\left(\mathrm{y}^{-1}\right)$ & $\mu+\tau\left(\mathrm{y}^{-1}\right)$ \\
\hline 300 & 21 & 1,3 & 0,9 & 2,2 \\
300 & 65 & 3,9 & 2,2 & 6,2 \\
\hline 600 & 21 & 0,5 & 0,3 & 0,7 \\
600 & 65 & 1,4 & 0,7 & 2,1 \\
\hline 900 & 21 & 0,2 & 0,1 & 0,3 \\
900 & 65 & 0,6 & 0,3 & 0,9 \\
\hline \multicolumn{5}{|c|}{$E_{\nu}>10^{8} \mathrm{GeV}$} \\
\hline $\mathrm{m}_{\tilde{q}}(\mathrm{GeV})$ & $\mathrm{V}\left(\mathrm{km}^{3}\right)$ & $\tau\left(\mathrm{y}^{-1}\right)$ & $\mu\left(\mathrm{y}^{-1}\right)$ & $\mu+\tau\left(\mathrm{y}^{-1}\right)$ \\
\hline 300 & 21 & 1,1 & 0,18 & 1,3 \\
300 & 65 & 3,1 & 0,6 & 3,7 \\
\hline 600 & 21 & 0,4 & 0,07 & 0,5 \\
600 & 65 & 1,1 & 0,2 & 1,3 \\
\hline 900 & 21 & 0,2 & 0,03 & 0,2 \\
900 & 65 & 0,5 & 0,1 & 0,6 \\
\hline
\end{tabular}


Em se tratando do decaimento de NLSPs na atmosfera o experimento adotado como referência é o JEM-EUSO, que deve ser instalado na estação espacial internacional. Destacamos que neste caso podemos ter até cerca de 30 decaimentos de taus em coincidência por ano para decaimentos de NLSPs com energias a partir de $10^{9} \mathrm{GeV}$ e até cerca de 0,2 decaimentos de taus em coincidência por ano para energias a partir de $10^{10} \mathrm{GeV}$, que corresponde ao limiar experimental. A área de detecção é de cerca de $2 \times 10^{5} \mathrm{~km}^{2}$ e a extensão de atmosfera considerada (altitude) foi de cerca de $100 \mathrm{~km}$. Para este experimento determinamos diversos fluxos na atmosfera: de NLSPs que passam pelo campo de visão do detector sem decair; de taus produzidos na atmosfera por meio do decaimento das NLSPs; de decaimentos em coincidência do par de staus produzidos na interação neutrino-núcleon; e a de decaimentos de taus na atmosfera, que também podem ser em coincidência. Para todas estas situações determinamos as respectivas distribuições de energia.

Para o caso de NLSPs passando pela atmosfera, temos uma limitação na produção de fluorescência atmosférica, pois sua intensidade está diretamente relacionada ao número de partículas carregadas que interagem com a atmosfera (equação 4.2). Isto ocorre porque a probabilidade de as NLSPs produzirem chuveiros atmosféricos é muita baixa [7], só restando a possibilidade de produção de fluorescência atmosférica por meio da ionização de partículas da atmosfera provocada apenas por sua passagem, visto que tratam-se de partículas carregadas. Sendo assim, os eventos mais promissores são aqueles gerados por decaimentos de taus ( $82 \%$ dos casos), para os quais podemos ter a produção de chuveiros atmosféricos. Neste caso avaliamos a taxa de eventos dentro do campo de visão do detector para diversos valores de $\sqrt{F}$. O valor para este parâmetro que maximiza a taxa de coincidências na atmosfera é $3 \times 10^{6} \mathrm{GeV}$, para o qual podemos ter até cerca de $2 \times 10^{5}$ coincidências por ano dentro do campo de visão do detector (tabela 4.1). Entretanto a energia das partículas que atingem a atmosfera neste caso se distribui em torno de $10^{5} \mathrm{GeV}$ (figura 4.4), valor muito aquém da energia mínima aceita pelo detector.

Quando impomos restrições de energia para os decaimentos de NLSPs na atmosfera, obtemos taxas significativas até mesmo para $E_{\tilde{\tau}} \geq 10^{10} \mathrm{GeV}$ (tabela 4.2), tendo mais limitações apenas para coincidências de decaimentos de taus. Temos uma quantidade significativa de decaimentos apenas para os 
casos em que não há coincidência. Ainda assim seriam $82 \%$ de 0,6 eventos por ano. A desvantagem neste caso reside no fato de serem eventos que seriam mais difíceis de distinguir de outros eventos raros que se espera observar neste experimento. Vale ressaltar que os resultados obtidos com cortes em energias mínimas para os decaimentos das NLSPs de $10^{8}$ e $10^{9} \mathrm{GeV}$ reforçam a discussão sobre experimentos cujas energias mínimas aceitas possam ser inferiores à do JEM-EUSO, lembrando que o volume de detecção adotado aqui é o deste experimento.

Para caracterizarmos os eventos na atmosfera com mais precisão, levantamos também quais seriam as distribuições da distância e do intervalo de tempo entre os decaimentos coincidentes. Observam-se que as distribuições são muito parecidas, mesmo quando são impostos cortes de energia para as coincidências dentro do campo de visão do detector. As distribuições nos dois casos nos mostram que podemos ter eventos muito particulares chegando ao campo de visão do detector, pois os dois chuveiros produzidos nas coincidências seriam vistos dentro dentro de um intervalo de tempo médio de $10^{-3,7} \mathrm{~s}$ e distância mais provável entre 50 e $100 \mathrm{~km}$, lembrando que a duração de um evento é de cerca de 0,3 ms e as resoluções temporal e espacial do detector são respectivamente de $2,5 \mu$ s e de $0,75 \mathrm{~km}$. 


\section{Capítulo 6}

\section{Conclusões}

Mostramos aqui que é possível detectar indiretamente partículas supersimétricas (sléptons) por meio de seu decaimento, o qual produz um tau mais um gravitino. Uma consequência importante é que nos cenários estudados, este decaimento está ligado a um intervalo restrito para a escala de quebra de supersimetria: $\sim 10^{5} \mathrm{GeV} \lesssim \sqrt{F} \lesssim 10^{7} \mathrm{GeV}$, que nos melhores cenários é ainda mais restrito: $\sim 10^{5} \mathrm{GeV} \lesssim \sqrt{F} \lesssim 10^{6} \mathrm{GeV}$. A observação indireta das NLSPs se daria por meio de sinais provocados pelo tau em telescópios de neutrinos ou de fluorescência atmosférica. Desta forma, propomos testes para observação de partículas supersimétricas bem como para a escala de quebra de supersimetria.

Para o caso de decaimentos de NLSPs no interior da Terra, temos a produção de taus que sofrem o processo de regeneração, o qual simulamos em concordância com trabalhos já publicados [28]. Para o telescópio IceCube, o número de eventos de taus é relativamente limitado, principalmente quando levamos em conta que apenas cerca de $20 \%$ deles podem deixar assinaturas claras. Quando consideramos volumes adicionais ao do IceCube podemos ter cerca de 1,5 evento por ano com assinatura bem definida do tau e 2 eventos por ano de múons acima do fundo de neutrinos atmosféricos. Lembramos que a taxa estimada para o fundo, de neutrinos atmosféricos, é superestimada nas escalas de energia que trabalhamos aqui, ou seja, nossos resultados são conservadores.

Quando o decaimento das partículas supersimétricas ocorre na atmosfera, determinamos as taxas em coincidência dos decaimentos dos taus, bem como 
as distribuições do intervalo de tempo e da distância entre eles, o que promove um conjunto de características únicas aos chuveiros atmosféricos produzidos. No caso do telescópio JEM-EUSO, as taxas máximas de coincidência dos decaimentos e do tau, dentro de seu campo de visão é muito elevada, podendo chegar a $2 \times 10^{5}$ eventos por ano, porém com energias em torno de $10^{5,5}$ $\mathrm{GeV}$, muito abaixo do limiar aceito pelo detector. Quando consideramos limiares mais elevados para a energia das NLSPs ao atingirem a atmosfera, continuamos tendo taxas significativas: podemos ter cerca de 30 decaimentos de taus em coincidência por ano para energias a partir de $10^{9} \mathrm{GeV}$ e até cerca de 0,2 decaimentos em coincidência por ano para energias a partir de $10^{10}$ $\mathrm{GeV}$, que corresponde ao limiar experimental.

Em resumo, é possível observar, nos melhores cenários, cerca de 1,5 assinaturas bem definidas de taus e 2 de múons em telescópios de neutrinos com $4 \mathrm{~km}$ de raio acrescidos ao raio do IceCube e cerca de 30 coincidências de decaimentos de taus com energia acima de $10^{9} \mathrm{GeV}$ no telescópio JEM-EUSO. 


\section{Apêndice A}

\section{Telescópio IceCube}

O telescópio de neutrinos IceCube, situado no pólo sul a uma profundidade de aproximadamente $2 \mathrm{~km}$, é o primeiro a ser construído na escala de $\mathrm{km}^{3}$ de volume. Ele possui capacidade de detectar neutrinos desde 100 $\mathrm{GeV}$ até neutrinos de ultra alta energia (EeV). Tecnicamente consiste em um conjunto de 5160 tubos (ver figura A.1) fotomultiplicadores (PMTs) envoltos em uma esfera transparente de pressão, compondo o chamado módulo óptico (MO) [36, 37]. São 86 cordas regularmente espaçadas de $125 \mathrm{~m}$ sobre uma área de aproximadamente $1 \mathrm{~km}^{2}$, com os MOs nas profundidades de 1,4 a 2,4 km abaixo da superfície. Cada corda possui uma sequência de 60 MOs espaçados de $17 \mathrm{~m}$. Os PMTs detectam fótons individuais de luz Cherenkov gerada no gelo por múons e elétrons.

O sinais de eventos neste detector consistem principalmente de múons que chegam a ele "de baixo para cima" (como na figura 1.1), produzidos em interações de neutrinos na rocha ou no gelo. Como a profundidade do detector (cerca de $2 \mathrm{~km}$ ) é muito pequena em relação ao tamanho da Terra, a taxa de eventos de interesse para ângulos menores que 90 graus é desprezível, pois a probabilidade de o neutrino produzir uma NLSP nestas condições é muito baixa. Além disso, a taxa de múons provenientes da interação de neutrinos atmosféricos compõe o principal fundo, sendo muito alta para ângulos menores que 90 graus.

Sinais de taus, quando produzidos ou quando decaem dentro do volume de detecção, podem ser dintinguidos de sinais de múons. Nas figuras A.2 e A.3 são ilustradas respectivamente topologias e simulações de eventos provocados 
por neutrinos do múon, elétron e tau.

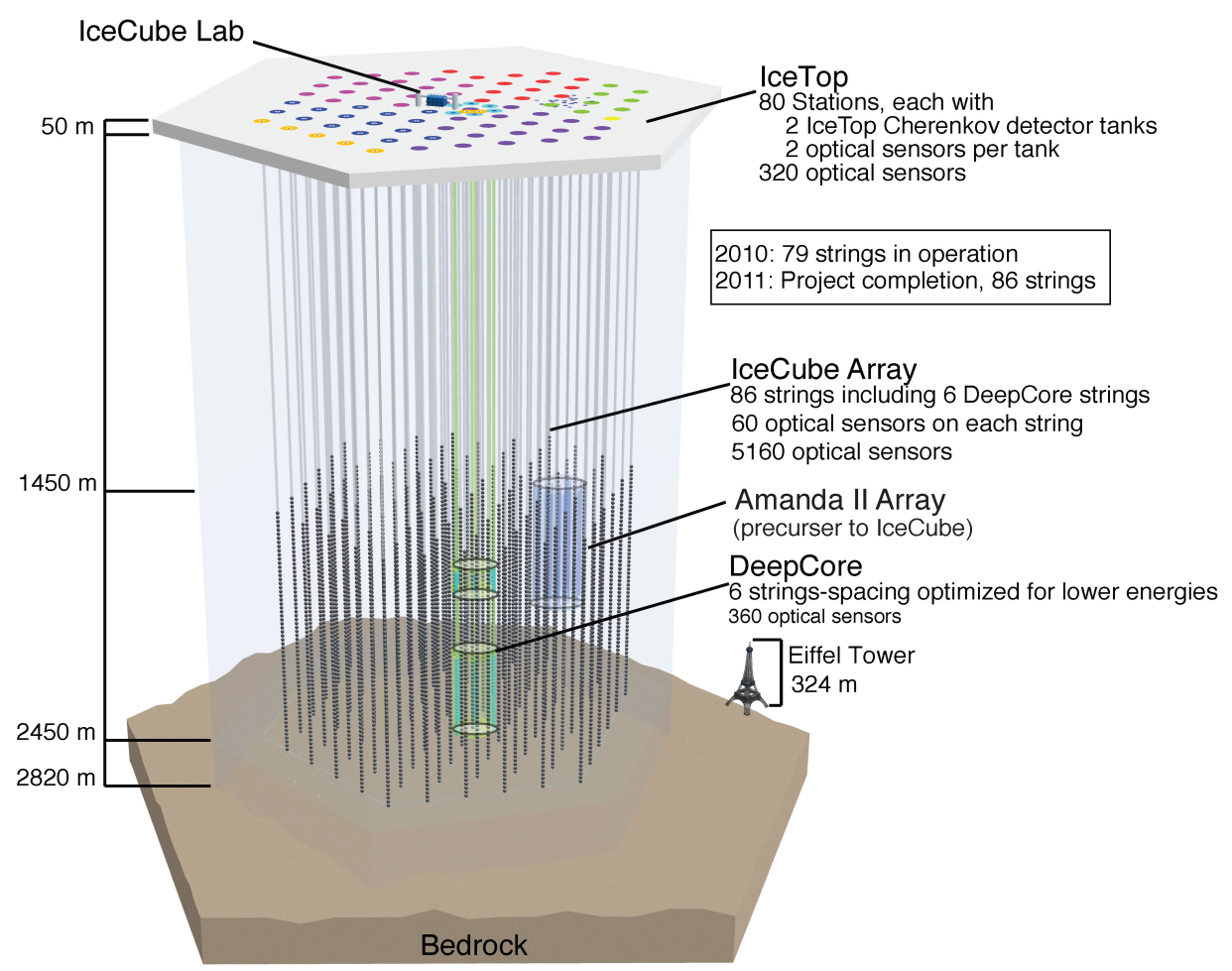

Figura A.1: Esquema do telescópio de neutrinos IceCube, em escala, obtido de [38].
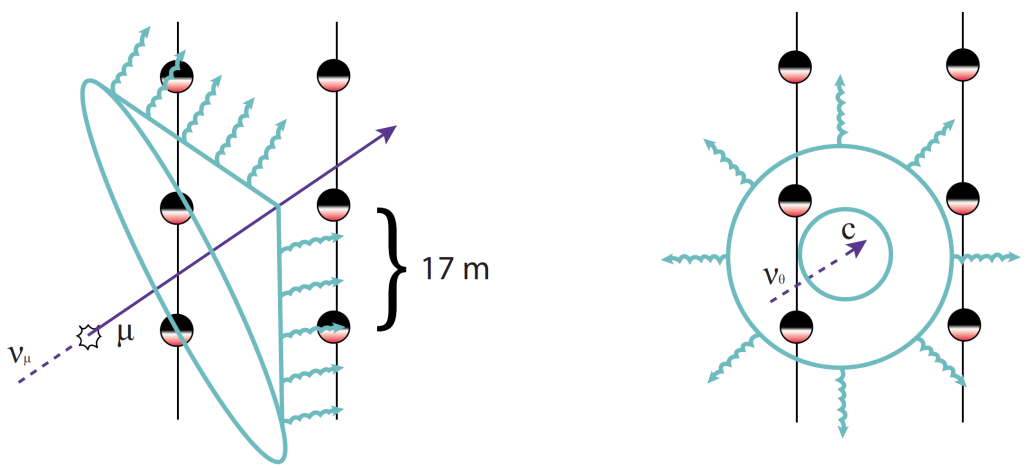

Figura A.2: À esquerda é ilustrado um sinal provocado por múons e à direita, pela interação do neutrino do elétron ou do tau. Figura btida de [36]. 
(a)

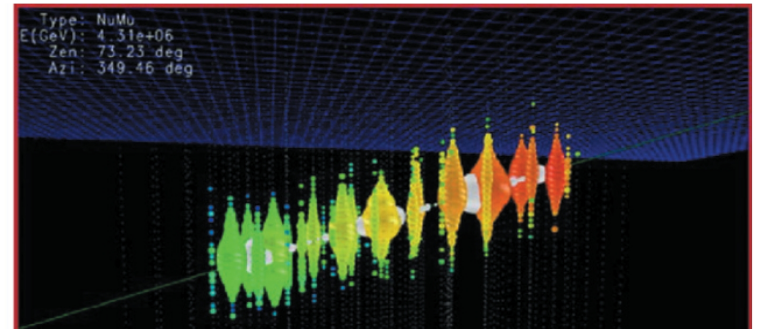

(b)

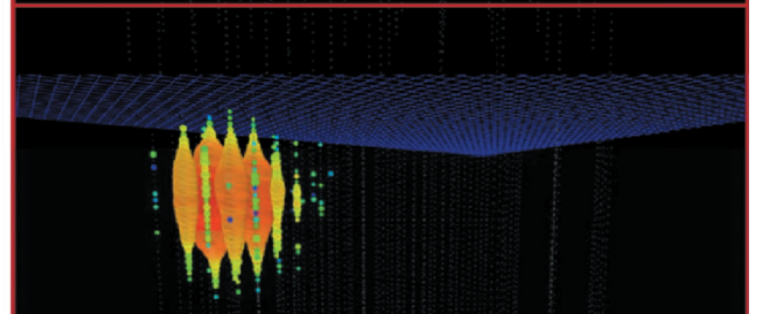

(c)

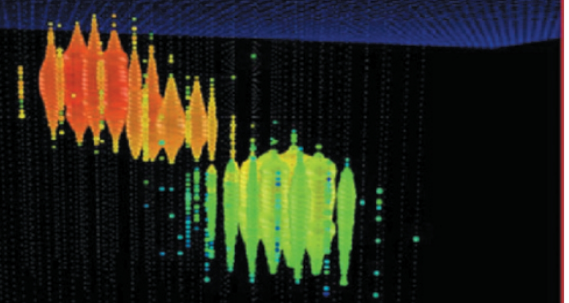

Figura A.3: Eventos simulados: (a) $\nu_{\mu} N \rightarrow \mu X$; (b) $\nu_{e} N \rightarrow$ cascata; (c) double bang, $\nu_{\tau} N \rightarrow$ cascata $_{1}+\nu_{t} a u N \rightarrow$ cascata $_{2}$. Figura obtida de [36]. 


\section{Apêndice B}

\section{Parametrizações}

Nesta seção são detalhadas as parametrizações utilizadas para se ganhar tempo computacional nas simulações. Para se ter um controle da qualidade das parametrizações, foi utilizada uma incerteza arbitrária para o ajuste pelo método dos mínimos quadrados, que basicamente era determinada pelo valor da função a ser parametrizada dividido por um fator escolhido arbitrariamente de forma que os critérios estabelecidos para aceitação do ajuste fossem satisfeitos. O primeiro deles era o valor do $\chi^{2}$ reduzido do ajuste próximo de 1; segundo: o gráfico de resíduos devia apresentar os pontos distribuídos aleatoriamente em torno de zero. Por fim, o terceiro critério utilizado foi o resíduo relativo, que consiste no resíduo absoluto divido pelo valor da função em cada ponto. Este valor não podia ser maior que 0,1\%. Este valor foi escolhido arbitrariamente.

Foram construídas tabelas com os parâmetros obtidos nas parametrizações. Estes parâmetros são apresentados com incertezas, provenientes dos ajustes, para se ter controle da precisão dos parâmetros frente aos valores obtidos dos cálculos realizados diretamente por meio das respectivas equações parametrizadas. Os critérios para controle das parametrizações mencionados acima foram utilizados nas parametrizações das seções B.1, B.2 e B.3, que são apresentadas a seguir. 


\section{B.1 Seção de choque - produção de léptons}

Na figura B.1 é apresentado um gráfico no qual os pontos em preto representam a seção de choque calculada e os em vermelho a curva ajustada. Foi necessário realizar um ajuste para cada década, caso contrário a convergência do ajuste não ocorria.

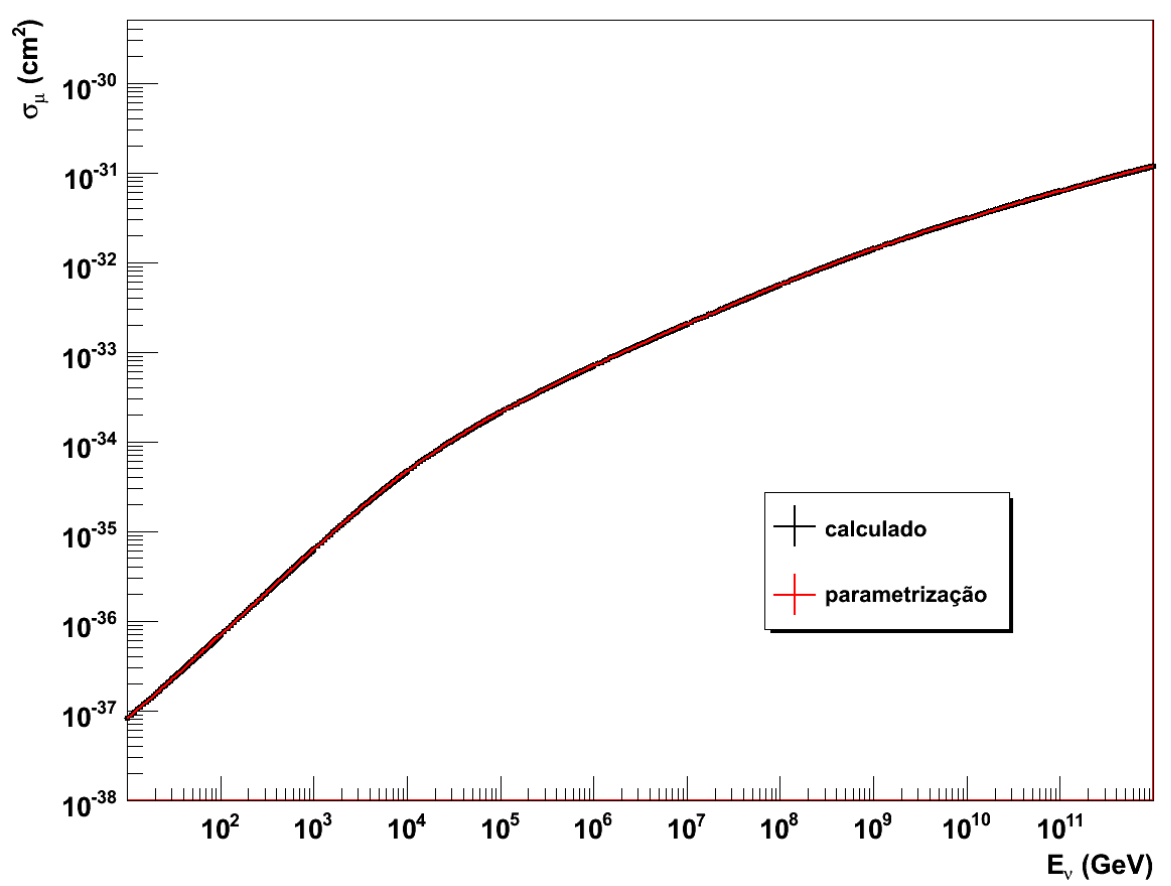

Figura B.1: Seção de choque inclusiva de corrente carrega da interação neutrinonucleon para a produção de múons. Em preto são apresentados os pontos obtidos com o cálculo da seção de choque e em vermelho, os pontos obtidos com a curva ajustada.

A função utilizada para o ajuste da seção de choque foi a seguinte:

$$
f=p_{0}+p_{1} \cdot E_{\nu}+p_{2} \cdot\left(E_{\nu}\right)^{p_{3}}
$$

Os parâmetros obtidos para a curva apresentada no gráfico da Figura B.1 são apresentados na Tabela B.1. 


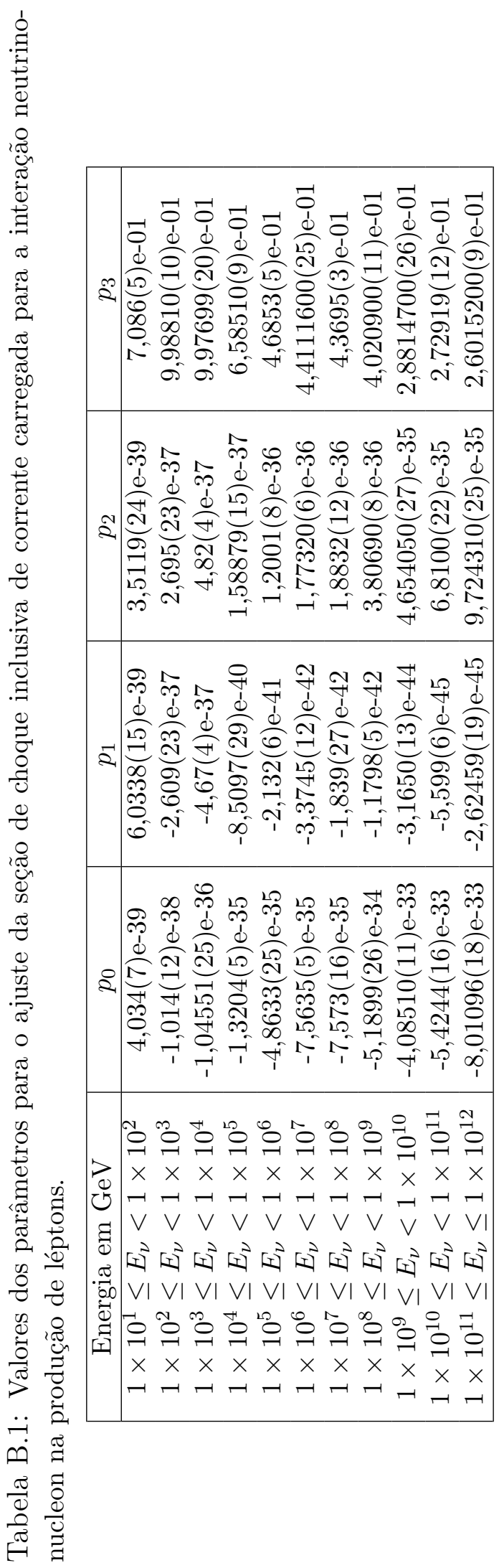




\section{B.2 Seção de choque - produção de NLSPs}

No caso da seção de choque de produção dos sléptons foram parametrizadas as três curvas apresentadas na Figura 2.4. É apresentado como exemplo a parametrização da seção de choque para massa do squark de $300 \mathrm{GeV}$ (Figura B.2).

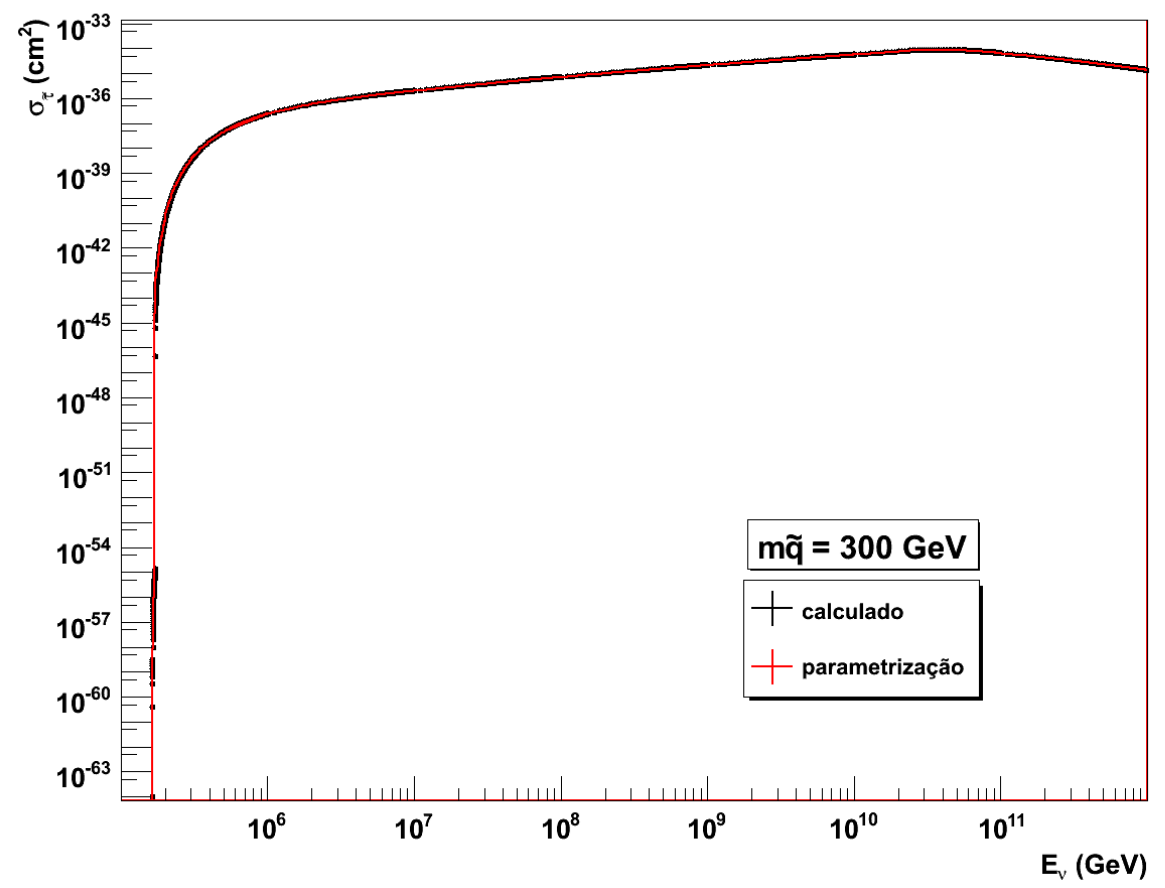

Figura B.2: Parametrização da seção de choque da interação neutrino-nucleon para a produção de partículas supersimétricas. Neste caso a massa do squark é $300 \mathrm{GeV}$.

A função ajustada para a curva apresentada na Figura B.2 é dada por:

$$
f=p_{0}+p_{1} \cdot\left(E_{\nu}\right)^{p_{2}}+p_{3} \cdot\left(E_{\nu}\right)^{p_{4}}
$$

Esta função foi ajustada aos valores da seção de choque para diversos intervalos de energia. Os parâmetros para este ajuste são apresentados na Tabela B.2. 


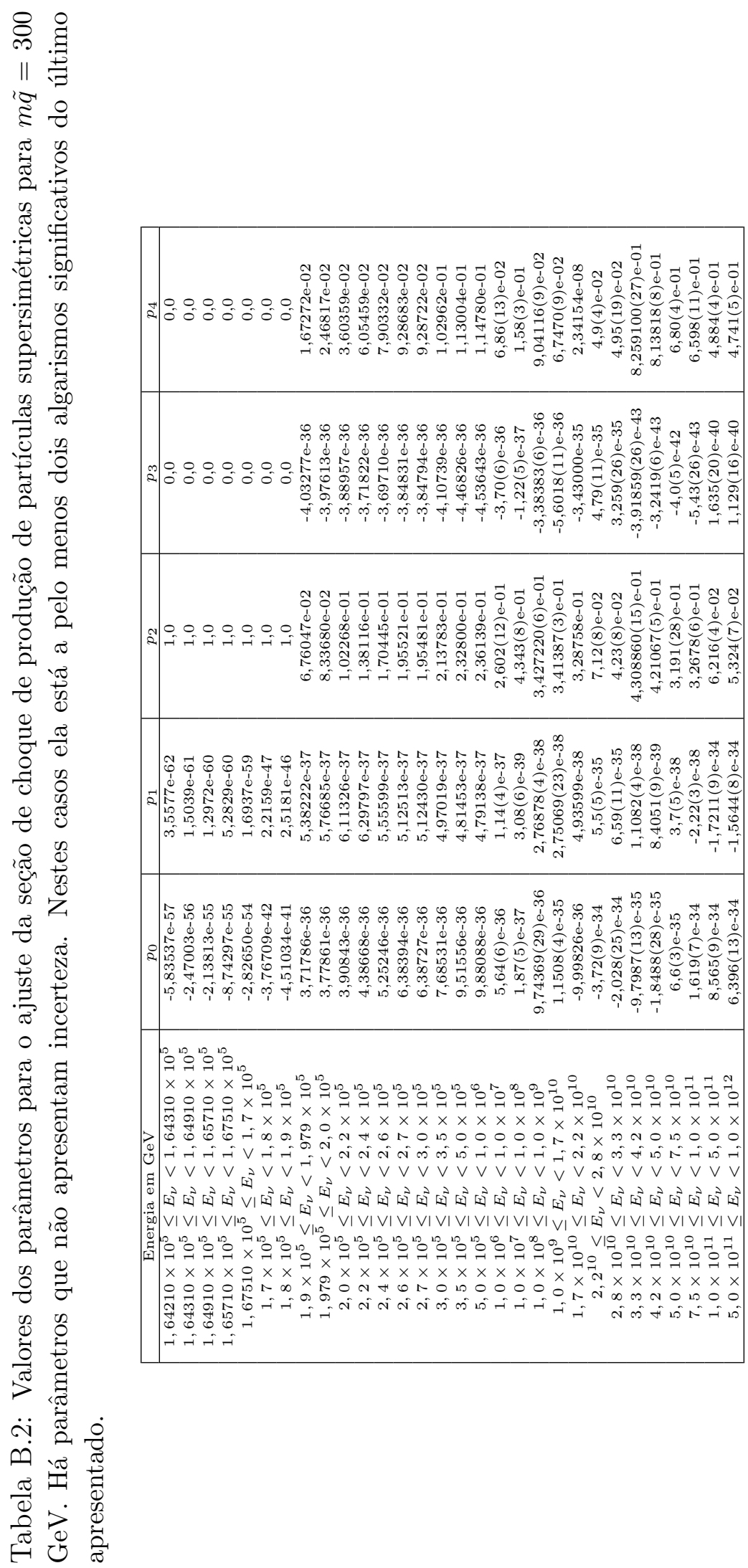




\section{B.3 Perda de energia: múon, tau e stau}

Nas Figuras 2.10, B.4 e 2.12 são apresentadas as parametrizações das somas das três contribuições para a perda de energia por radiação. Para o primeiro caso, as partículas em questão são múons, no segundo são taus e no terceiro caso, sléptons de massa de repouso de $150 \mathrm{GeV}$.

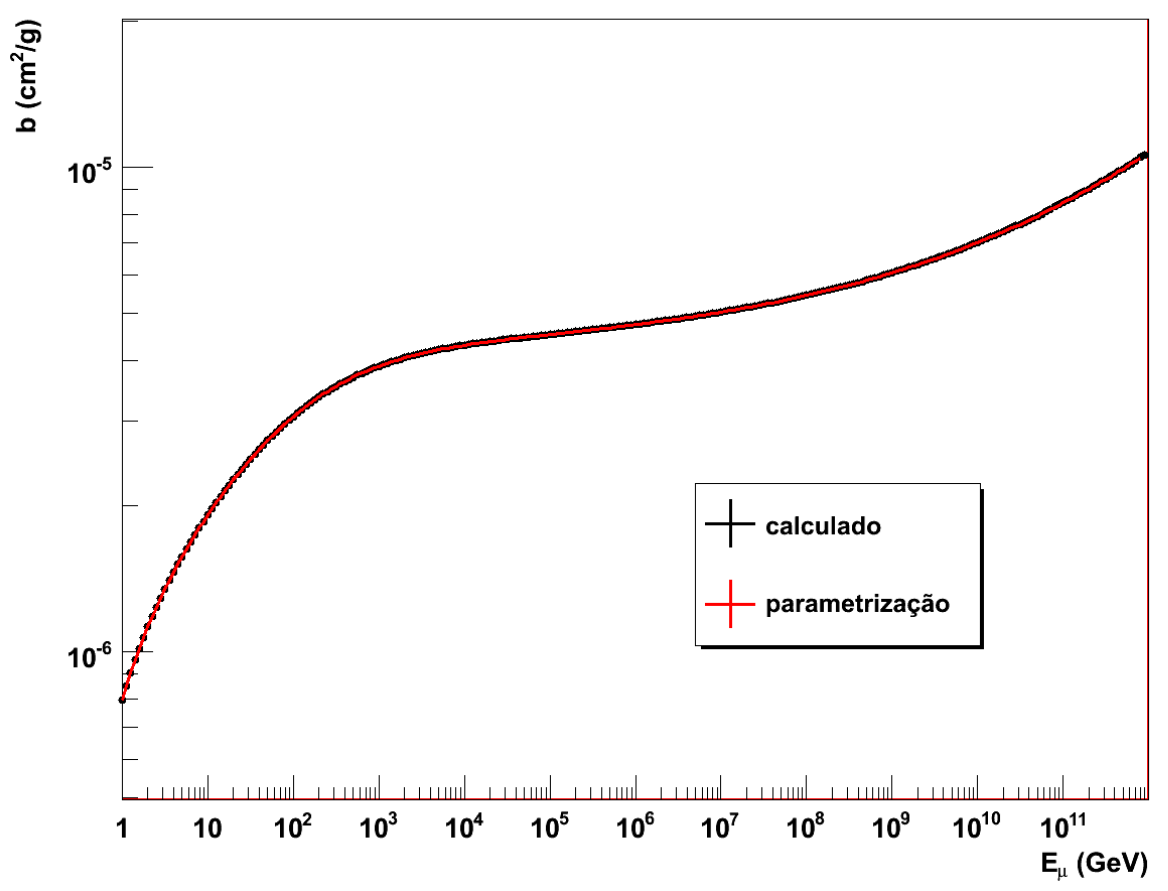

Figura B.3: Parametrização da perda de energia (por radiação) de múons ao atravessar a Terra em função da energia de incidência. É apresentada a soma das contribuições relevantes: por bremsstrahlung, por produção de pares e por interação fotonuclear.

A função utilizada para a perda de energia de múons, para energias entre $1 \mathrm{GeV}$ e $1 \times 10^{12} \mathrm{GeV}$, é dada por:

$$
f=p_{0}+p_{1} \cdot E_{\mu}+p_{2} \cdot\left(E_{\mu}\right)^{2}+p_{3} \cdot\left(E_{\mu}\right)^{p_{4}}
$$

Os parâmetros dos ajustes para a perda de energia do múon são apresentados na Tabela B.3. 


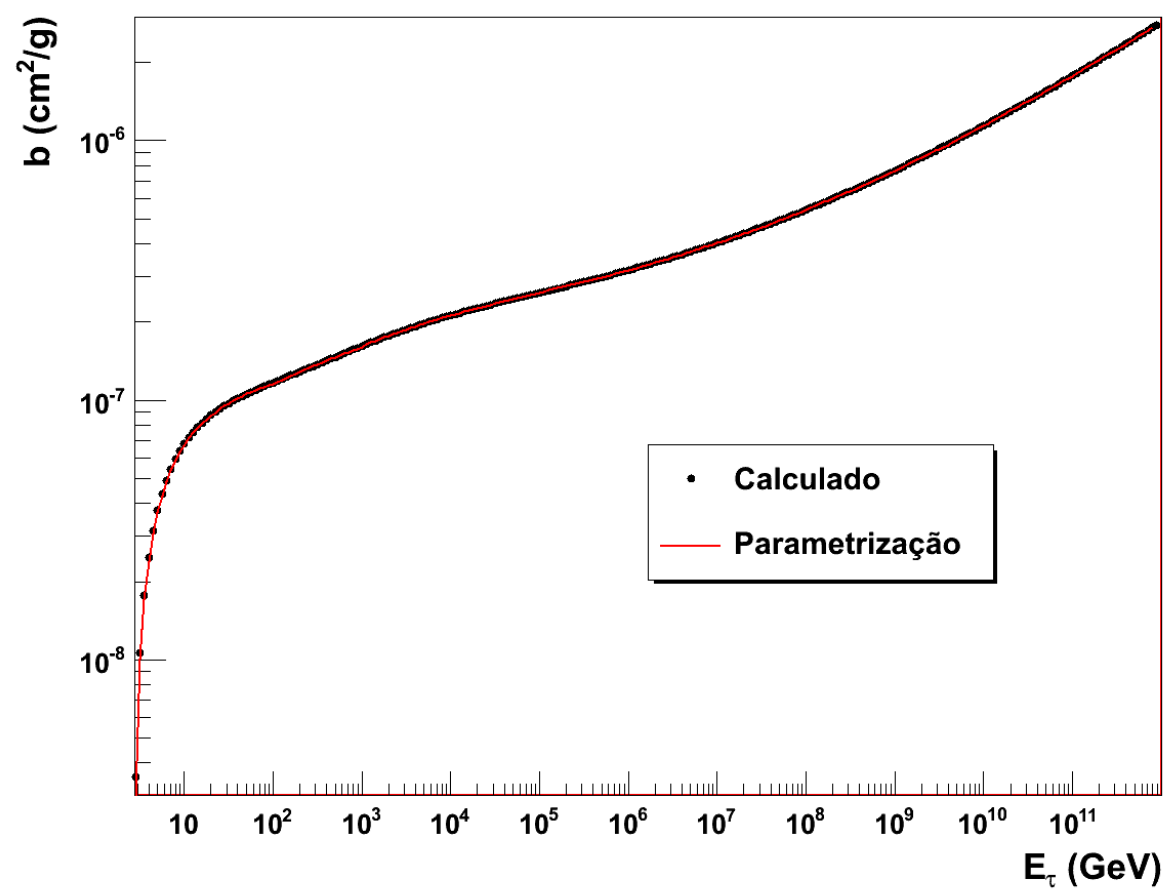

Figura B.4: Parametrização da perda de energia (por radiação) de taus ao atravessar a Terra em função da energia de incidência. É apresentada a soma das contribuições relevantes: por bremsstrahlung, por produção de pares e por interação fotonuclear.

A função utilizada para a perda de energia dos taus, para energias entre 2, $8 \mathrm{GeV}$ e $1 \times 10^{12} \mathrm{GeV}$, é dada por:

$$
f=p_{0}+p_{1} \cdot\left(E_{\tau}\right)^{p_{2}}+p_{3} \cdot\left(E_{\tau}\right)^{p_{4}}
$$




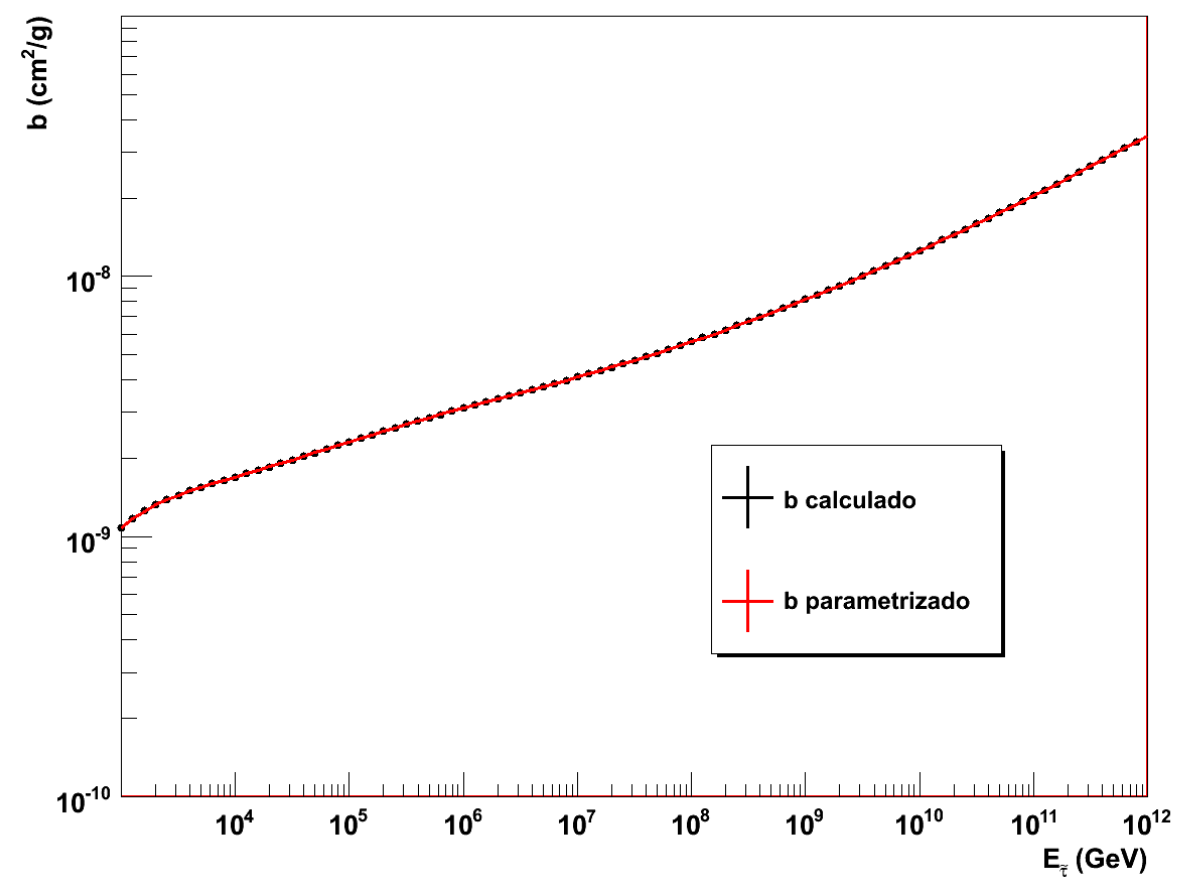

Figura B.5: Parametrização da perda de energia (por radiação) do slépton de 150 $\mathrm{GeV}$ ao atravessar a Terra em função da energia de incidência. É apresentada a soma das contribuições relevantes: por bremsstrahlung, por produção de pares e por interação fotonuclear.

A função utilizada para parametrizar a perda de energia dos sléptons é dada por:

$$
p_{0}+p_{1} \cdot\left(E_{\tilde{l}}\right)^{p_{2}}+p_{3} \cdot\left(E_{\tilde{l}}\right)^{p_{4}}+p_{5} \cdot\left(E_{\tilde{l}}\right)^{p_{6}}
$$

Os parâmetros obtidos são apresentados na Tabela B.5. 


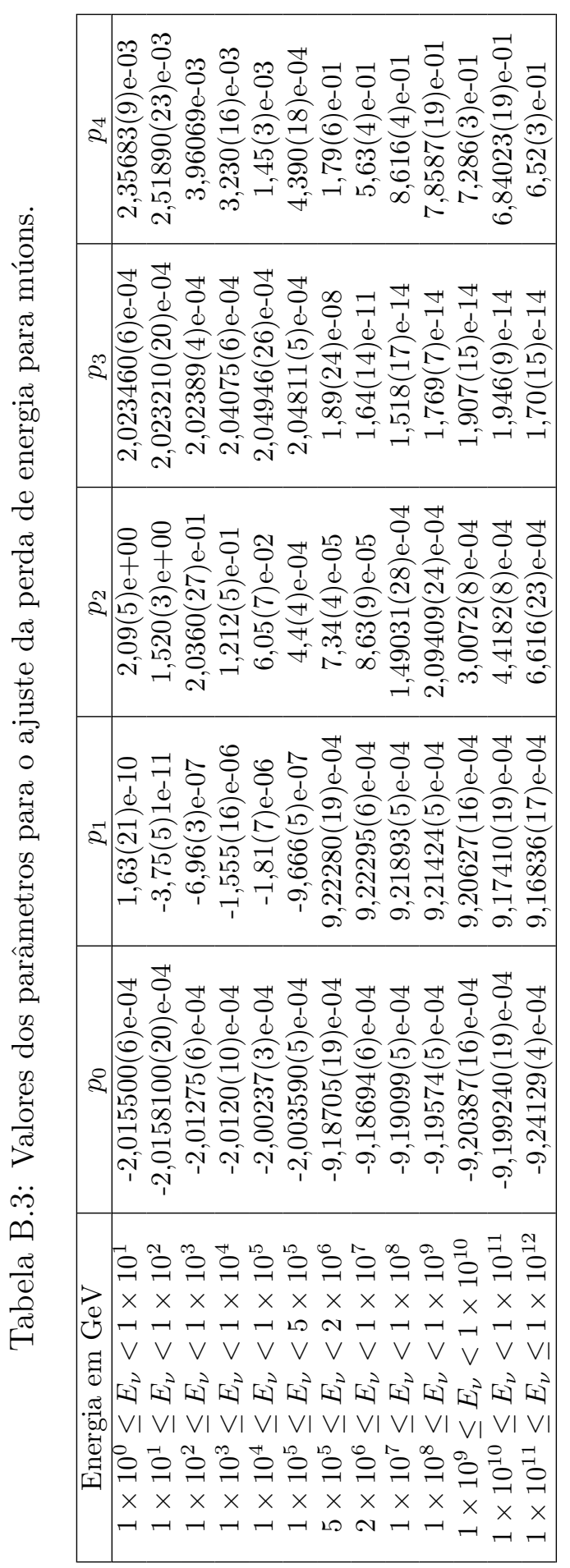




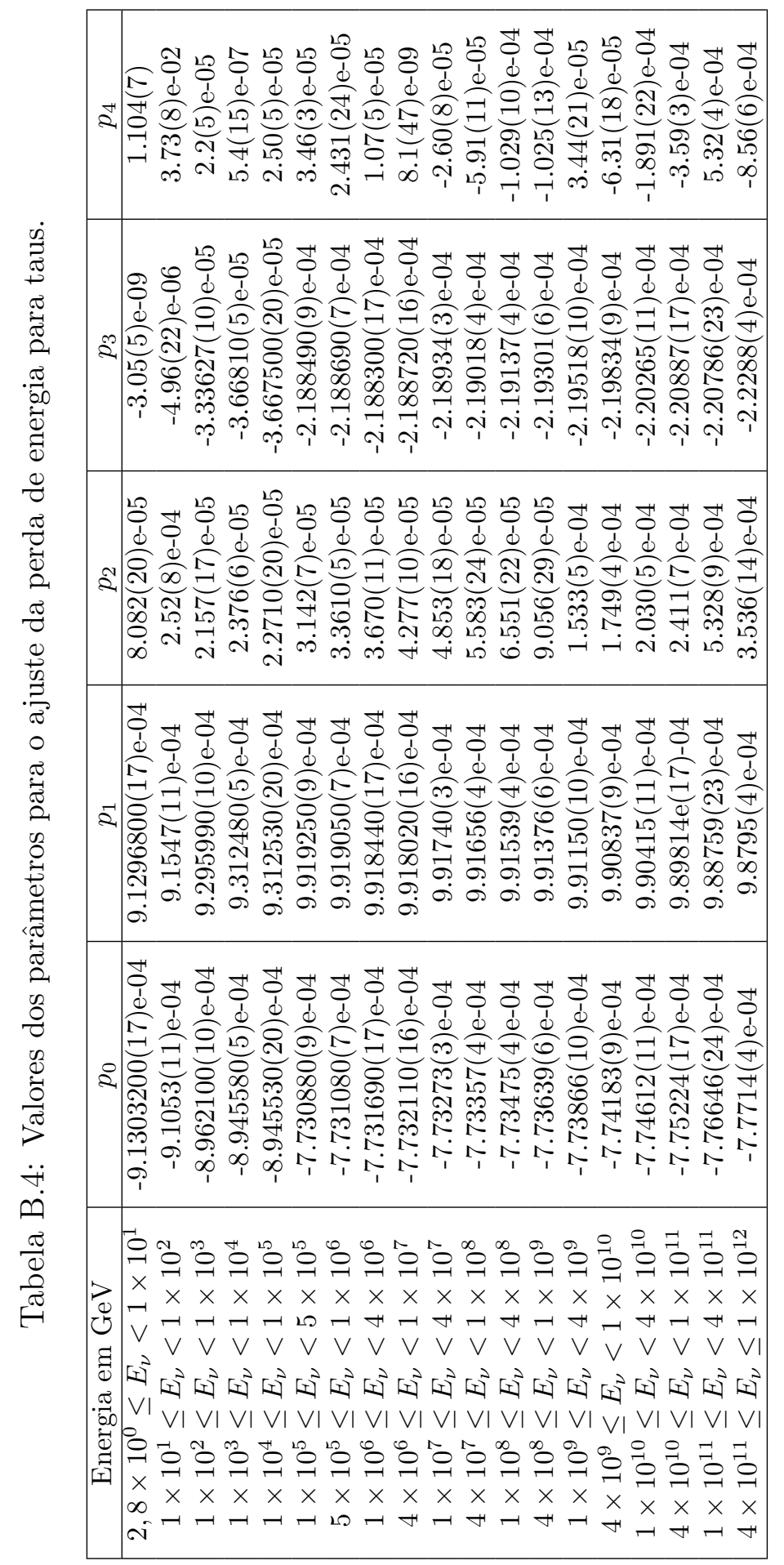



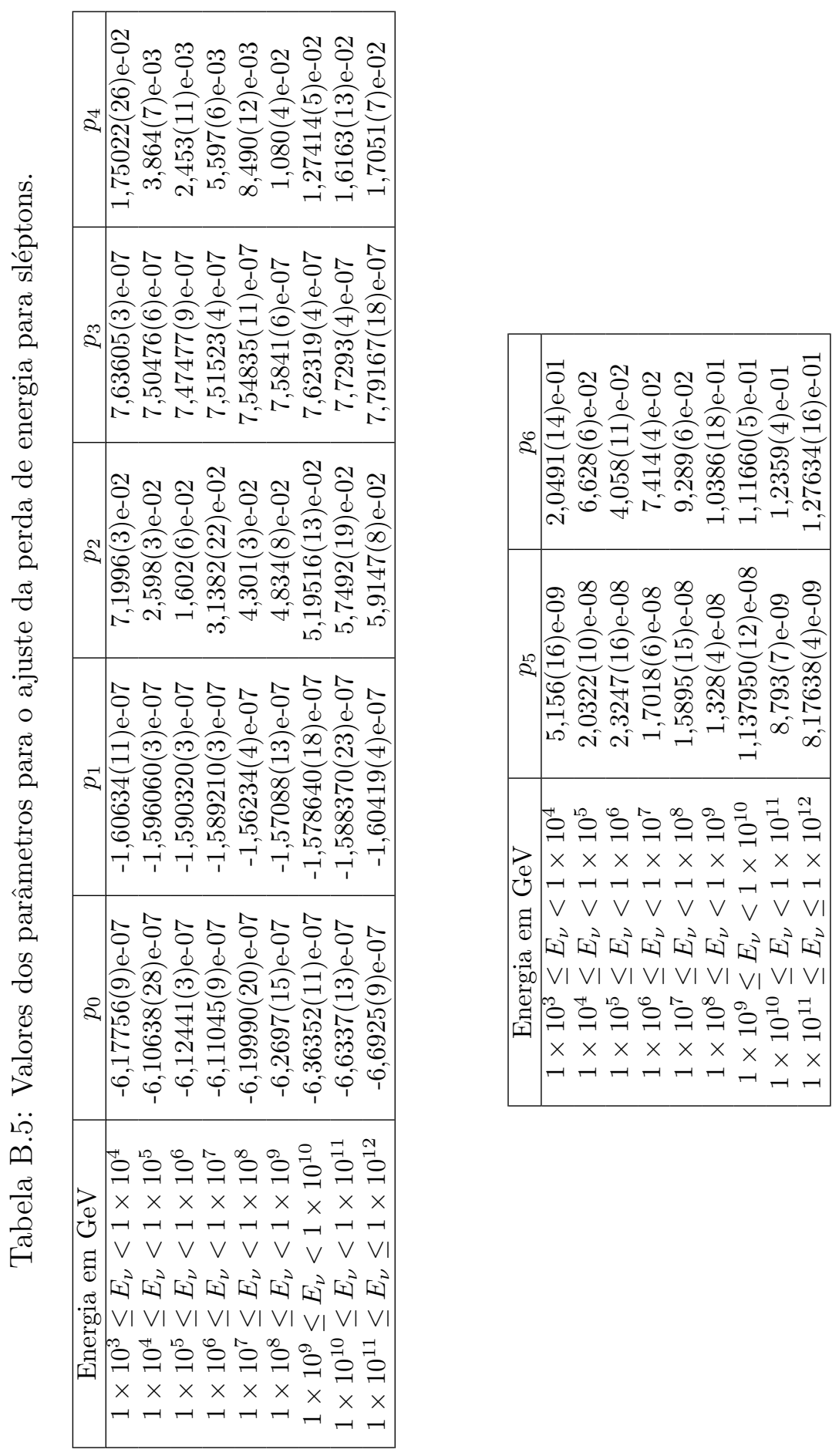


\section{B.4 Parametrização de y}

A fração de energia perdida na interação neutrino-núcleon quando ocorre a produção de um lépton é dada por:

$$
y=\frac{E_{\nu}-E_{l}}{E_{\nu}}
$$

Portanto a energia do múon em função da energia do neutrino e de $y$ é dada por:

$$
E_{l}=(1-y) E_{\nu}
$$

Foram utilizados os valores para y (corrente carregada) encontrados na tabela 1 da referência [14], apresentados na tabela B.6. A parametrização neste caso consistiu em uma interpolação linear dos pares ordenados $\left(E_{\nu}, y\right)$.

Tabela B.6: Valores de $y$ em função de $E_{\nu}$. Dados de [14].

\begin{tabular}{|c|c|}
\hline$E_{\nu}(\mathrm{GeV})$ & $y_{c c}$ \\
\hline $1 \times 10^{1}$ & 0,483 \\
$1 \times 10^{2}$ & 0,477 \\
$1 \times 10^{3}$ & 0,472 \\
$1 \times 10^{4}$ & 0,426 \\
$1 \times 10^{5}$ & 0,332 \\
$1 \times 10^{6}$ & 0,273 \\
$1 \times 10^{7}$ & 0,250 \\
$1 \times 10^{8}$ & 0,237 \\
$1 \times 10^{9}$ & 0,225 \\
$1 \times 10^{10}$ & 0,216 \\
$1 \times 10^{11}$ & 0,208 \\
$1 \times 10^{12}$ & 0,205 \\
\hline
\end{tabular}

Na figura B.6 é mostrado um gráfico da curva obtida com a interpolação linear e os pontos correspondentes aos pares ordenados da tabela B.6. No gráfico, nota-se que a curva interpolada não aparenta ser uma reta. Isto ocorre devido ao fato de a escala do eixo da energia do neutrino estar logarítimica. 


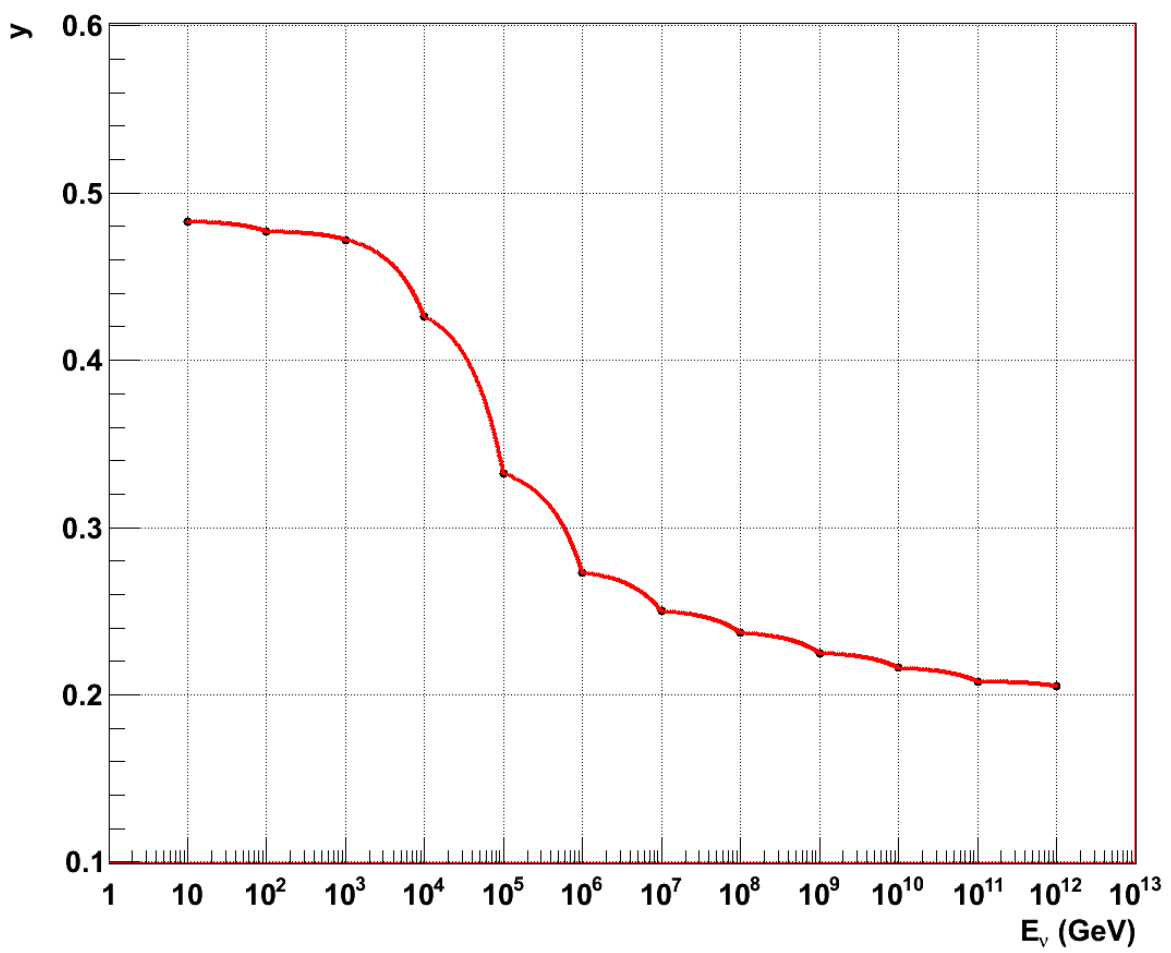

Figura B.6: Parametrização de y. 


\section{Apêndice C}

\section{Números aleatórios seguindo uma distribuição de probabilidades qualquer}

$\mathrm{Na}$ simulação, para gerar aleatoriamente, por exemplo, o valor de $\theta_{C M}$, ângulo entre uma das partículas geradas no decaimento do stau e a direção de propagação do mesmo, o precedimento é bem simples, pois no centro de momentos esse ângulo é uniformemente distribuído [29]. Desta forma, basta usar uma função que gere números aleatórios distribuídos uniformemente entre 0 e 1 e multiplicar o resultado pelo intervalo de abrangência do ângulo $\theta_{C M}$.

Por outro lado, para determinar, por exemplo, o ponto de interação entre o neutrino incidente e um nucleon dentro da Terra, é necessário gerar números aleatórios que sigam a distribuição de probabilidade de o neutrino interagir (equação 2.18) ao passar pela Terra e produzir as partículas de interesse (NLSPs) neste ponto dentro do planeta. Neste caso, a distribuição não é uniforme e muda para cada ângulo/energia de incidência do neutrino.

Neste sentido, foi desenvolvido um algoritmo Monte Carlo para gerar números aleatórios que seguem uma distribuição qualquer partindo-se do gerador de números aleatórios uniformemente distribuídos, tornando possível determinar, por exemplo, em qual ponto da Terra ocorre a interação neutrinonúcleon, ou ainda, determinar a energia do neutrino do tau produzido no decaimento do tau. 
Um exemplo para verificar o funcionamento do algoritmo de distribuição de probabilidades quaisquer é apresentado na Figura C.1. O gráfico representa a distribuição de probabilidade dada pela equação (C.1) para um neutrino que incide sobre a Terra, com um ângulo e uma energia de incidência fixos.

$$
P=P s_{\nu}\left(1-P s_{\nu \tilde{l}}\right)
$$

em que $P s_{\nu}$ é a probabilidade de sobrevivência do neutrino. $\left(1-P s_{\nu \tilde{l}}\right)$ é a probabilidade de, na interação do neutrino com a Terra, produzir-se um slépton.

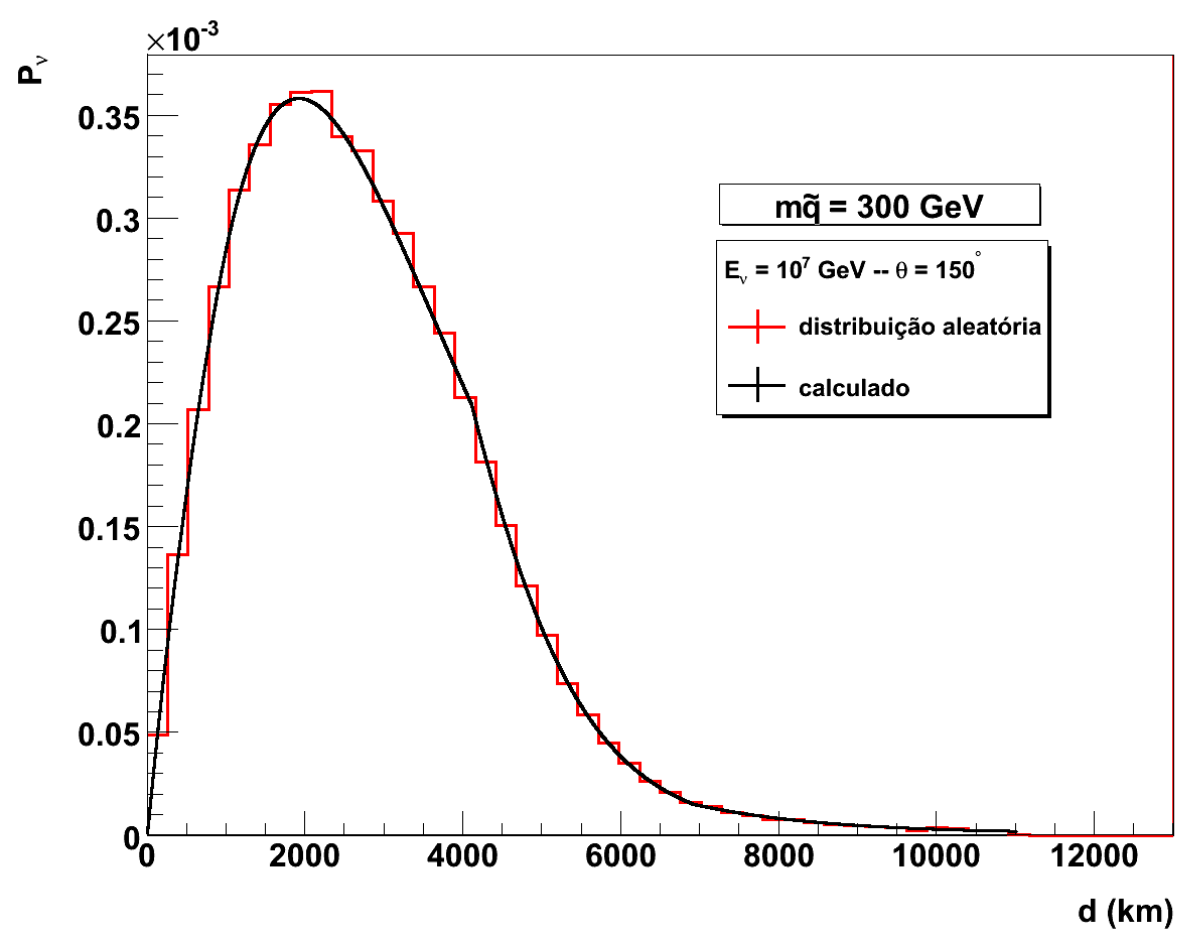

Figura C.1: Comparação entre o histograma (em vermelho) obtido com o algoritmo criado para gerar números aleatórios que seguem uma distribuição qualquer e a distribuição de probabilidade dada por $p=p_{s_{\nu}}\left(1-p_{s_{\nu}}\right)$, em função da distância entre a superfície (no ponto de incidência) e o detector. A curva em preto é a distribuição calculada analiticamente.

No caso da Figura C.1 a energia de incidência do neutrino é de $1 \times 10^{7}$ $\mathrm{GeV}$ e o ângulo de incidência é de 150 graus. A linha preta é a distribuição 
de probabilidades definida previamente e o histograma em vermelho foi obtido gerando-se números aleatórios que seguissem a distribuição dada. O histograma foi normalizado pela probabilidade em questão para facilitar a comparação. Nota-se um bom acordo entre a curva e o histograma. A flutuação do histograma em torno da curva é dependente do número de eventos gerados.

Outro exemplo é a distribuição $\frac{d n}{d z}$ utilizada para determinar a energia do neutrino do tau a parti do decaimento do tau.

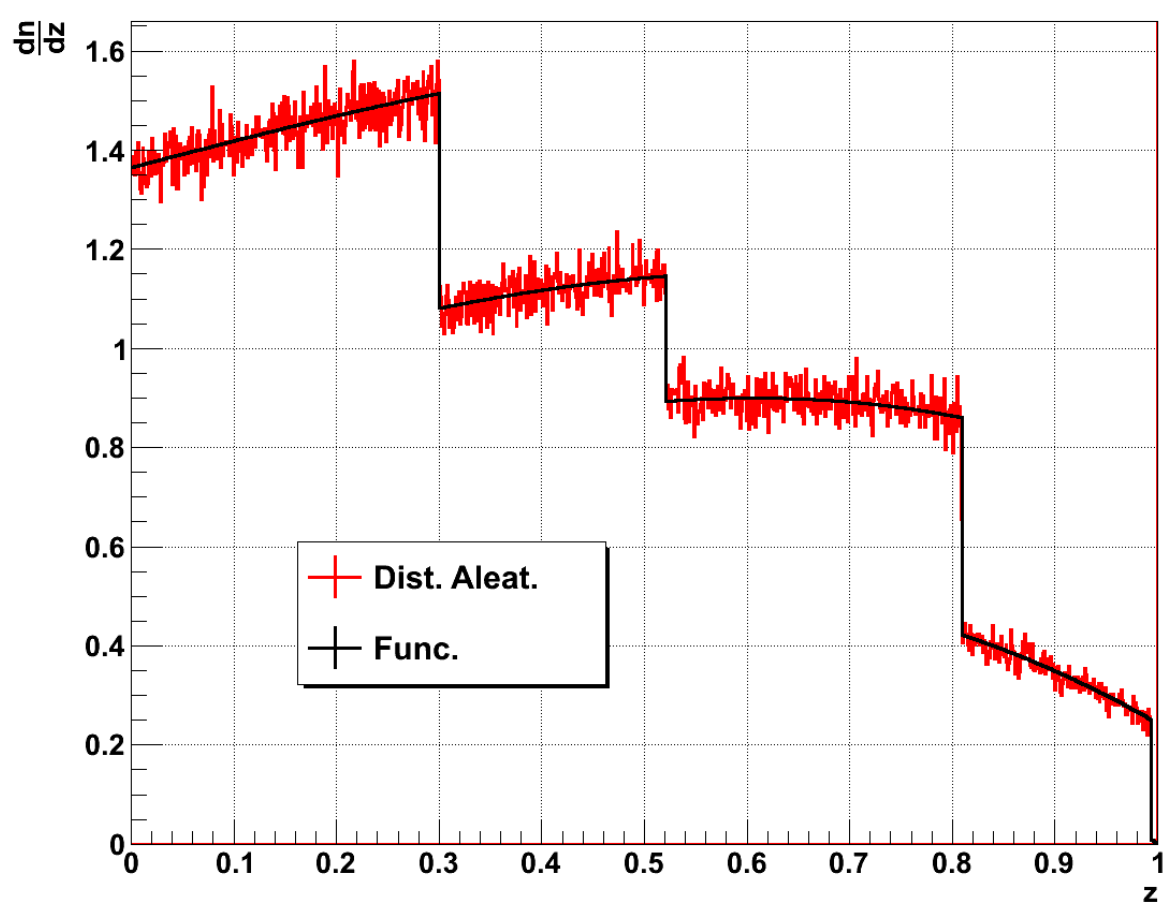

Figura C.2: Distribuição $\frac{d n}{d z}$ em função de $z$. Em preto tem-se a função dada pela equação (3.11) e em vermelho, a respectiva distribuição aleatória.

O algoritmo utilizado consiste em discretizar a distribuição de probabilidades utilizada para sortear os números aleatórios, alocando cada inifinitésimo de sua área em um vetor, da seguinte maneira, supondo a distribuição de probabilidades dada por $f(x)$, com $x \in[a, b]$ : dividimos o interavalo $[a, b]$ em $N$ partes iguais de tamanho $\Delta x$ e calculamos as $N$ áreas $A_{i}=\Delta x . f\left(x_{i}\right)$ sob a curva $f(x)$. Determinamos, então, a área mínima den- 
tre as $N$ áreas e a tomamos como área unitária. Armazenamos as $N$ áreas num vetor $y[N]$ e os $N$ valores de $x$ corresondentes, em um vetor $X[N]$. A partir disto, geramos um novo vetor da distribuição em si, Dist $[M]$, e para cada posição $X[i]$ determinamos a quantas áreas mínimas corresponde cada área $y[i]$. Assim alocamos $k$ posições do vetor Dist com o valor da posição $x[i]$. Finalmente, para gerar um número aleatório que siga esta distribuição, basta sortear um número aleatório entre 0 e $M$, a partir de uma distribuição uniforme, e verificar a qual posição do vetor Dist este número corresponde. 


\section{Apêndice D}

\section{Cinemática}

Neste apêndice são apresentadas algumas deduções de cinemática relativística relacionadas às situações físicas simuladas. Na seção D.2 é mostrado como é obtida a energia, no referencial do laboratório, de uma das partículas produzidas no decaimento em dois corpos. Esta relação é utilizada no decaimento do stau. Já na seção D.3 é mostrado como é obtida a relação entre o ângulo no referencial do centro de momentos e o correspondente no referencial do laboratório.

\section{D.1 Convenções adotadas}

As variáveis com apóstrofe (') representam grandezas no referencial do centro de momentos. Variáveis em negrito representam trivetores. Variáveis escalares e quadrivetores são denotados por letras em itálico.

O sistema de unidades adotado é tal que $\hbar=c=1$. Desta forma, temos a seguinte relação entre as dimensões:

$$
[\text { comprimento }]=[\text { tempo }]=[\text { energia }]^{-1}=[\text { massa }]^{-1} \text {. }
$$

A seguir são apresentadas as convenções relativísticas adotadas.O tensor métrica é dado por: 


$$
g_{\mu \nu}=g^{\mu \nu}=\left(\begin{array}{cccc}
1 & 0 & 0 & 0 \\
0 & -1 & 0 & 0 \\
0 & 0 & -1 & 0 \\
0 & 0 & 0 & -1
\end{array}\right)
$$

onde os índices representados por letras gregas correspondem a 0, 1, 2, 3 ou $t, x, y, z$. Índices romanos, e.g. $i, j$, denotam apenas as componentes espaciais. Índices repetidos representam somas.

A velocidade é definida por:

$$
\mathbf{p}=m \gamma \mathbf{v}
$$

com

$$
\gamma=\frac{1}{\sqrt{1-v^{2}}}
$$

A energia de uma partícula é dada por:

$$
E=m \gamma
$$

O quadrimomento é dado por:

$$
P=(E, \mathbf{p})
$$

O produto escalar entre dois quadrimomentos é dado por:

$$
P_{1} \cdot P_{2}=E_{1} E_{2}-\mathbf{p}_{1} \cdot \mathbf{p}_{2}
$$

Uma consequência direta é o módulo do quadrimomento:

$$
P^{2}=P^{\mu} P_{\mu}=E^{2}-|\mathbf{p}|^{2}=m^{2}
$$

Transformações de Lorentz para energia e momento, variáveis no referencial do laboratório em função de variáveis do CM:

$$
E=\gamma\left(E^{\prime}+v p_{x}^{\prime}\right)
$$




$$
p_{x}=\gamma\left(p_{x}^{\prime}+v E^{\prime}\right)
$$

\section{D.2 Decaimento em dois corpos}

Consideremos uma partícula de massa $M$ com energia $E_{L a b}$ no referencial do laboratório e em repouso no referencial do centro de momentos (CM). Ao decair, esta partícula produz duas novas partículas, com massas $m_{1}$ e $m_{2}$. O objetivo aqui é obter a energia da partícula 2 no referencial do laboratório, em função das grandezas conhecidas: $E_{L a b}, m_{1}$ e $m_{2}$.

No centro de momentos devemos ter a conservação do quadrimomento:

$$
P^{\prime}=P_{1}^{\prime}+P_{2}^{\prime}
$$

Isolando-se $P_{1}^{\prime}$ e calculando-se sua intensidade, temos:

$$
P_{1}^{\prime}=P^{\prime}-P_{2}^{\prime} \Rightarrow P_{1}^{\prime 2}=\left(P^{\prime}-P_{2}^{\prime}\right)^{2}=P^{\prime 2}+P_{2}^{\prime 2}-2 P^{\prime} \cdot P_{2}^{\prime}
$$

Usando o fato de que $P^{\prime 2}=m^{2}$ (equação D.7) na igualdade anterior, temos:

$$
m_{1}^{2}=M^{2}+m_{2}^{2}-2 P^{\prime} \cdot P_{2}^{\prime}
$$

com $P^{\prime} \cdot P_{2}^{\prime}=E^{\prime} E_{2}-\mathbf{p}^{\prime} \cdot \mathbf{p}_{\mathbf{2}}^{\prime}$ (equação D.6). Mas, $\mathbf{p}^{\prime}=0$, pois a partícula que decai está em repouso no CM. Portanto, $P^{\prime} . P_{2}^{\prime}=E^{\prime} E_{2}^{\prime}$. Assim, a igualdade anterior fica da seguinte maneira:

$$
m_{1}^{2}=M^{2}+m_{2}^{2}-2 E^{\prime} E_{2}^{\prime}
$$

De $\mathbf{p}^{\prime}=0$ obtem-se também:

$$
E^{\prime 2}-\left(\mathbf{p}^{\prime}=0\right)^{2}=M^{2} \Rightarrow E^{\prime}=M
$$

Substituindo-se o resultado da equação D.11 em D.10 e isolando-se $E_{2}^{\prime}$, temos:

$$
E_{2}^{\prime}=\frac{M^{2}+m_{2}^{2}-m_{1}^{2}}{2 M}
$$


Para obter a energia no referencial do laboratório, basta aplicar uma transformação de Lorentz (equação D.8):

$$
E_{2_{L a b}}=\gamma_{C M}\left[E_{2}^{\prime}+v_{C M} p_{2}^{\prime} \cos \theta_{C M}\right]
$$

$\operatorname{com} \gamma_{C M}=E_{L a b} / M$, obtido a partir da equação (D.4).

$v_{C M}$ é obtido a partir da definição de $\gamma$ (equação D.3):

$$
\gamma_{C M}=\frac{1}{\sqrt{1-v_{C M}^{2}}}
$$

Para obter $v_{C M}$, basta isolá-lo na equação acima, o que resulta em:

$$
v_{C M}=\sqrt{1-1 / \gamma_{C M}^{2}}
$$

Para finalizar, $p_{2}^{\prime}$ é dado por:

$$
p_{2}^{\prime}=\frac{1}{2 M} \sqrt{\left[M-\left(m_{1}+m_{2}\right)^{2}\right]\left[M-\left(m_{1}-m_{2}\right)^{2}\right]}
$$

A dedução de $p_{2}^{\prime}$ é mostrada na seção D.3.3. Este resultado é equivalente ao da equação D.39.

\section{D.3 Boost do CM para Lab}

A determinação da relação entre os ângulos no CM e no Lab é duduzida com basa no esquema apresentado na figura D.1. Primeiramente são determinadas as relações entre $\alpha$ e $\theta$, a partir da conservação do momento no CM. 


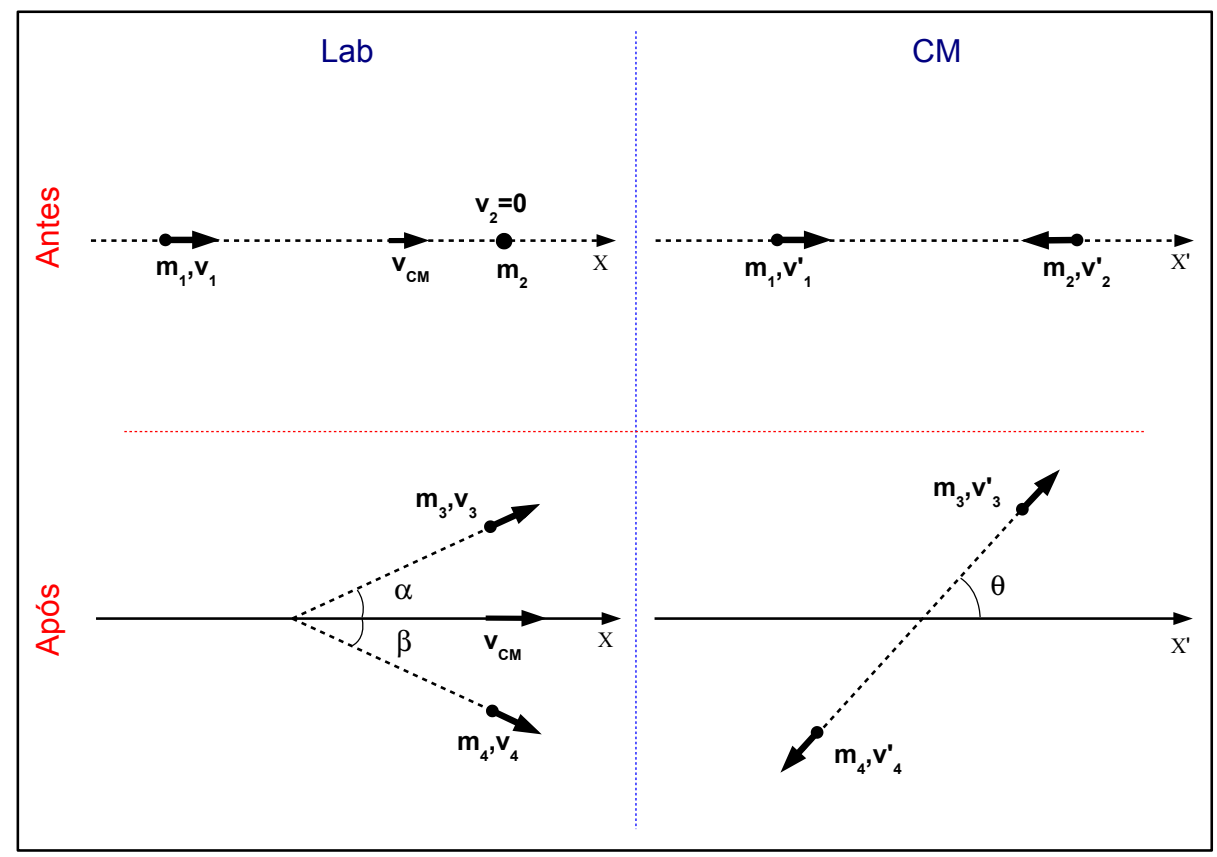

Figura D.1: Esquema da colisão entre as partículas 1 e 2, produzindo após a colisão, as partículas 3 e 4. A situação é ilustrada nos referenciais do laboratório (Lab) e do centro de momentos (CM).

No CM, $p_{3}^{\prime}=p_{4}^{\prime}$, pois o momento total no CM é zero. Como o deslocamento entre os referenciais ocorre na direção $x$ (não há momento na direção $y$ antes da colisão). Sendo assim temos:

$$
\mathbf{p}_{3}+\mathbf{p}_{4}=0
$$

As transformações de Lorentz na direção y são, então, dadas por:

$$
\begin{aligned}
& p_{3_{y}}=p_{3_{y}}^{\prime} \\
& p_{4_{y}}=p_{4_{y}}^{\prime}
\end{aligned}
$$

Assim, usando a equação D.2 temos que:

$$
p_{3_{y}}=p_{3 y}^{\prime}=m_{3} \gamma_{3}^{\prime} v_{3_{y}}^{\prime}=m_{3} \gamma_{3}^{\prime} v_{3}^{\prime} \sin \theta
$$

No caso dos momentos na direção $x$ : 


$$
p_{3_{x}}=\gamma_{C M}\left(p_{3_{x}}^{\prime}+v_{C M} E_{3}^{\prime}\right)
$$

$\operatorname{com} E_{3}^{\prime}=m_{3} \gamma_{3}^{\prime}$.

Usando as equações D.2 e D.4, temos:

$$
\begin{aligned}
p_{3_{x}} & =\gamma_{C M}\left(m_{3} \gamma_{3}^{\prime} v_{3_{x}}^{\prime}+v_{C M} m_{3} \gamma_{3}^{\prime}\right) \\
& =\gamma_{C M}\left[m_{3} \gamma_{3}^{\prime} v_{3}^{\prime} \cos \theta+v_{C M} m_{3} \gamma_{3}^{\prime}\right] \\
& =m_{3} \gamma_{C M} \gamma_{3}^{\prime}\left[v_{3}^{\prime} \cos \theta+v_{C M}\right]
\end{aligned}
$$

O ângulo $\alpha$ pode ser definido da seguinte maneira:

$$
\tan \alpha=\frac{p_{3_{y}}}{p_{3 x}}
$$

Substituindo-se as equações D.18 e D.20 em D.21, temos:

$$
\tan \alpha=\frac{\sin \theta}{\gamma_{C M}\left(\cos \theta+v_{C M} / v_{3}^{\prime}\right)}
$$

Logo, $\alpha$ é dado por:

$$
\alpha=\arctan \left[\frac{\sin \theta}{\gamma_{C M}\left(\cos \theta+v_{C M} / v_{3}^{\prime}\right)}\right] .
$$

$\gamma_{C M}$ e $v_{C M}$ são determinados (em função de parâmetros conhecidos) na seção D.3.1 e $v_{3}^{\prime}$, na seção D.3.2.

$\mathrm{O}$ ângulo $\beta$ pode ser determinado de forma similar a $\alpha$. Neste caso os momentos envolvidos são os da partícula 4, cujo ângulo no CM é dado por $\theta+\pi$, pois as partículas se propagam em direções opostas neste referencial.

$$
p_{4_{y}}=p_{4_{y}}^{\prime}=m_{4} \gamma_{4}^{\prime} v_{4_{y}}^{\prime}=m_{4} \gamma_{4}^{\prime} v_{4}^{\prime} \sin (\theta+\pi)
$$

Na direção $x$ temos a transformação de Lorentz para a partícula 4 , dada por:

$$
p_{4_{x}}=\gamma_{C M}\left(p_{4_{x}}^{\prime}+v_{C M} E_{4}^{\prime}\right)
$$

$\operatorname{com} E_{4}^{\prime}=m_{4} \gamma_{4}^{\prime}$.

Novamente, usando as equações D.2 e D.4: 


$$
\begin{gathered}
p_{4_{x}}=\gamma_{C M}\left(m_{4} \gamma_{4}^{\prime} v_{4_{x}}^{\prime}+v_{C M} m_{4} \gamma_{4}^{\prime}\right) \\
=\gamma_{C M}\left[m_{4} \gamma_{4}^{\prime} v_{4}^{\prime} \cos (\theta+\pi)+v_{C M} m_{4} \gamma_{4}^{\prime}\right], \\
=m_{4} \gamma_{C M} \gamma_{4}^{\prime}\left[v_{4}^{\prime} \cos (\theta+\pi)+v_{C M}\right] \\
\tan \beta=\frac{p_{4_{y}}}{p_{4_{x}}}, \\
\tan \beta=\frac{\sin (\theta+\pi)}{\gamma_{C M}\left[\cos (\theta+\pi)+v_{C M} / v_{4}^{\prime}\right]} .
\end{gathered}
$$

Finalmente, $\beta$ é dado por:

$$
\beta=\arctan \left\{\frac{\sin (\theta+\pi)}{\gamma_{C M}\left[\cos (\theta+\pi)+v_{C M} / v_{4}^{\prime}\right]}\right\} .
$$

Assim como gamma $a_{C M}, v_{C M}$ e $v_{3}^{\prime}$, $v_{4}^{\prime}$ também é determinada (em função de parâmetros conhecidos) mais adiante, na seção D.3.2. Outros parâmetros importantes tais como os momentos no $\mathrm{CM}$ e a energia do $\mathrm{CM}$, dos quais os ângulos no laboratório dependem, são determinados nas seções D.3.3 e D.3.4, respectivamente.

\section{D.3.1 Determinação de $\gamma_{C M}$ e de $v_{C M}$}

Aplicando a transformação de Lorentz (equação D.8) para as partículas 1 e 2 chega-se ao seguinte:

$$
\begin{aligned}
& E_{1}=\gamma_{C M}\left(E_{1}^{\prime}+v_{C M} p_{1_{x}}^{\prime}\right) \\
& E_{2}=\gamma_{C M}\left(E_{2}^{\prime}+v_{C M} p_{2_{x}}^{\prime}\right)
\end{aligned}
$$

Ao somar estas duas equações, tem-se:

$$
E_{1}+E_{2}=\gamma_{C M}\left[E_{1}^{\prime}+E_{2}^{\prime}+v_{C M}(\underbrace{p_{1_{x}}^{\prime}+p_{2_{x}}^{\prime}}_{0})\right]
$$

Mas $E_{1}^{\prime}+E_{2}^{\prime}=\sqrt{s}$ (equação D.34) e $E_{2}=m_{2}$, pois a partícula 2 está em repouso no referencial do laboratório. Dessa forma, temos:

$$
E_{1}+m_{2}=\gamma_{C M} \sqrt{s}
$$

Logo: 


$$
\gamma_{C M}=\frac{E_{1}+m_{2}}{\sqrt{s}}
$$

Para determinar $v_{C M}$, basta utilizar a definição de $\gamma$ dada pela equação D.3 na equação D.30 e isolar $v_{C M}$ :

$$
\frac{1}{\sqrt{1-v_{C M}}}=\frac{E_{1}+m_{2}}{\sqrt{s}}
$$

Elevando-se ao quadrado ambos os membros da igualdade acima e substituindose $s$ pelo resultado obtido na seção D.3.4 (equação D.44), temos:

$$
v_{C M}=\frac{\sqrt{E_{1}^{2}-m_{1}^{2}}}{E_{1}+m_{2}}
$$

\section{D.3.2 Determinação das velocidades no CM}

É possível escrever $v_{3}^{\prime}$ e $v_{4}^{\prime}$ em função do momento final, visto que

$$
\left|\mathbf{p}_{3}^{\prime}\right|=\left|\mathbf{p}_{4}^{\prime}\right|=p_{f}^{\prime}=p^{\prime}=\left|\mathbf{p}_{1}^{\prime}\right|=\left|\mathbf{p}_{2}^{\prime}\right| \text {. }
$$

Ou seja, $p^{\prime}$ e $p_{f}^{\prime}$ são os módulos dos momentos das partículas no CM antes e após a colisão, respectivamente.

Usando as equações D.2 e D.3 para a partícula 3, temos:

$$
p_{3}^{\prime}=m_{3} \gamma_{3}^{\prime} v_{3}^{\prime}=p^{\prime} \Rightarrow p^{\prime}=\frac{m_{3} v_{3}^{\prime}}{\sqrt{1-v_{3}^{\prime 2}}}
$$

Elevando-se ao quadrado o último resultado para $p^{\prime}$ e isolando-se $v_{3}^{\prime}$, temos:

$$
v_{3}^{\prime}=\frac{p^{\prime}}{\sqrt{m_{3}^{2}+p^{\prime 2}}}
$$

Da mesma forma, temos um resultado similar para a partícula 4:

$$
v_{4}^{\prime}=\frac{p^{\prime}}{\sqrt{m_{4}^{2}+p^{\prime 2}}}
$$

em que $p^{\prime}$ é determinado na seção D.3.3, dado pela equação D.39. 


\section{D.3.3 Determinação dos momentos no CM}

No CM, temos a seguinte igualdade:

$$
s=\left(P_{1}^{\prime}+P_{2}^{\prime}\right)^{2}=\left(P_{3}^{\prime}+P_{4}^{\prime}\right)^{2}
$$

A seguir é mostrado o cálculo de $p^{\prime}$ em função das partículas 1 e 2 , porém o resultado é completamente equivalente para $p_{f}^{\prime}$, em função das partículas 3 e 4 .

Usando a definição de quadrimomento (equação D.5) :

$$
s=\left(P_{1}^{\prime}+P_{2}^{\prime}\right)^{2}=\left(P_{3}^{\prime}+P_{4}^{\prime}\right)^{2}=\left[\left(E_{1}^{\prime}, \mathbf{p}_{1}^{\prime}\right)+\left(E_{2}^{\prime}, \mathbf{p}_{2}^{\prime}\right)\right]^{2}
$$

Como $\mathbf{p}_{1}^{\prime}+\mathbf{p}_{2}^{\prime}=0$, temos:

$$
s=\left[E_{1}^{\prime}+E_{2}^{\prime}\right]^{2} \Rightarrow \sqrt{s}=E_{1}^{\prime}+E_{2}^{\prime}
$$

Da equação D.7 e lembrando que $\left|\mathbf{p}_{1}^{\prime}\right|=\left|\mathbf{p}_{2}^{\prime}\right|=p^{\prime}$ :

$$
\begin{aligned}
& E_{1}^{\prime 2}-\mathbf{p}_{1}^{\prime 2}=m_{1}^{2} \Rightarrow E_{1}^{\prime}=\sqrt{m_{1}^{2}+\mathbf{p}_{1}^{\prime 2}} \Rightarrow E_{1}^{\prime}=\sqrt{m_{1}^{2}+p^{\prime 2}} \\
& E_{2}^{\prime 2}-\mathbf{p}_{2}^{\prime 2}=m_{2}^{2} \Rightarrow E_{2}^{\prime}=\sqrt{m_{2}^{2}+\mathbf{p}_{2}^{\prime 2}} \Rightarrow E_{2}^{\prime}=\sqrt{m_{2}^{2}+p^{\prime 2}}
\end{aligned}
$$

Substituindo-se os últimos termos dos resultados acima para $E_{1}^{\prime}$ e $E_{2}^{\prime}$ em D.36:

$$
\begin{aligned}
& \sqrt{s}=\sqrt{m_{1}^{2}+p^{\prime 2}}+\sqrt{m_{2}^{2}+p^{\prime 2}} \\
\Rightarrow \quad & \left(\sqrt{s}-\sqrt{m_{1}^{2}+p^{\prime 2}}\right)^{2}=\left(\sqrt{m_{2}^{2}+p^{\prime 2}}\right)^{2}
\end{aligned}
$$

Após manipulações algébricas, chega-se a:

$$
s+m_{1}^{2}-m_{2}^{2}=2 \sqrt{s} \sqrt{m_{1}^{2}+p^{\prime 2}}
$$

Elevando-se ambos os membros ao quadrado e isolando-se $p^{\prime}$, temos:

$$
\begin{aligned}
p^{\prime 2} & =\frac{1}{4 s}\left(s+m_{1}^{2}-m_{2}^{2}\right)^{2}-m_{1}^{2} \\
& =\frac{1}{4 s}\left(s^{2}-2 s\left(m_{1}^{2}+m_{2}^{2}\right)+\left(m_{1}^{2}-m_{2}^{2}\right)^{2}\right.
\end{aligned}
$$

Após completar quadrados somando-se e subtraindo-se $\left(m_{1}^{2}+m_{2}^{2}\right)$, chega- 
se em:

$$
p^{\prime 2}=\frac{1}{4 s}\left[s-\left(m_{1}^{2}+m_{2}^{2}\right)\right]^{2}-\left(2 m_{1} m_{2}\right)^{2}
$$

Finalmente, após duas fatorações de produtos notáveis, temos que $p^{\prime}$ é dado por:

$$
p^{\prime}=\frac{1}{2 \sqrt{s}} \sqrt{\left[s-\left(m_{1}-m_{2}\right)^{2}\right]\left[s-\left(m_{1}+m_{2}\right)^{2}\right]}
$$

Para as partículas 3 e 4 a solução é equivalente, com o seguinte resultado:

$$
p_{f}^{\prime}=\frac{1}{2 \sqrt{s}} \sqrt{\left[s-\left(m_{3}-m_{4}\right)^{2}\right]\left[s-\left(m_{3}+m_{4}\right)^{2}\right]}
$$

Na seção D.3.4, s é determinado em função de grandezas conhecidas no referencial do laboratório, $m_{1}, m_{2}$ e $E_{1}$.

\section{D.3.4 Determinação da energia do CM}

No referencial do laboratório temos a seguinte igualdade:

$$
s=\left(P_{1}+P_{2}\right)^{2}=\left(P_{3}+P_{4}\right)^{2},
$$

onde $s$ pode ser completamente determinado a partir das partículas 1 e 2 , pois suas as energias são conhecidas, bem como suas massas. A partícula 2 está em repouso antes da colisão.

$$
\begin{gathered}
s=\left(P_{1}+P_{2}\right)^{2}=\left[\left(E_{1}, p_{1_{x}}, 0,0\right)+\left(m_{2}, 0,0,0\right)\right] \\
s=\left(E_{1}+m_{2}\right)^{2}-\mathbf{p}_{1}^{2} \\
=E_{1}^{2}+2 m_{2} E_{1}+m_{2}^{2}-\mathbf{p}_{1}^{2}
\end{gathered}
$$

Mas, da equação D.7, temos que:

$$
E_{1}^{2}-\mathbf{p}_{1}^{2}=m_{1}^{2} \Rightarrow \mathbf{p}_{1}^{2}=E_{1}^{2}+m_{1}^{2}
$$

Substituindo-se D.43 em D.42, temos finalmente:

$$
s=m_{1}^{2}+m_{2}^{2}+2 m_{2} E_{1}
$$




\section{Apêndice E}

\section{Simulação de trajetórias}

Para simular as trajetórias das partículas em três dimensões, nos volumes dos detectores IceCube e JEM-EUSO, partimos de dois pontos da superfície formada pelo volume de detecção, sendo o primeiro passo determiná-los.

No caso do IceCube, cuja geometria foi aproximada por um clindro, os dois pontos são sorteados, apartir de distribuições uniformes, na superfície lateral e/ou nas superfícies inferior e superior. Para tomar a decisão em relação a qual superfície o ponto sorteado pertence, foram criados três intervalos numéricos arbitrariamente delimitados pelas frações das áreas das três superfícies em questão, relativas à area total. Sendo $A_{1}, A_{2}$ e $A_{3}$ as áreas da superfície superior, lateral e inferior, respectivamente, e sendo a $A_{t}$ a área total dada por:

$$
A_{t}=A_{1}+A_{2}+A_{3}
$$

as frações das áreas são dadas por:

$$
a_{1}=A_{1} / A_{t} ; a_{2}=A_{2} / A_{t} ; a_{3}=A_{3} / A_{t}
$$

É sorteado, então, um número $\xi$ uniformemente distribuído entre 0 e 1. De acordo com o intervalo, definido abaixo, temos a superfície à qual o ponto pertence: 


$$
\begin{array}{r}
0 \leq \xi<a_{1} \\
a_{1} \leq \xi<a_{1}+a_{2} \\
a_{1}+a_{2} \leq \xi \leq 1
\end{array}
$$

No caso do JEM-EUSO, o primeiro ponto é sorteado sempre na superfície da Terra e o segundo, pode ser na superfície lateral do tronco de cone (figura 4.2) ou na superior, delimitada pela altitude máxima da atmosfera (figura 4.1). Ou seja, o número aleatorio $\xi$ é comparado a apenas dois interavalos:

$$
\begin{aligned}
& 0 \leq \xi<a_{1}^{\prime} \\
& a_{1}^{\prime} \leq \xi \leq 1
\end{aligned}
$$

com as frações das áreas dadas por:

$$
a_{1}^{\prime}=A_{1}^{\prime} / A_{t}^{\prime} ; \quad a_{2}^{\prime}=A_{2}^{\prime} / A_{t}^{\prime}
$$

onde a área total é definida da seguinte forma:

$$
A_{t}^{\prime}=A_{1}^{\prime}+A_{2}^{\prime}
$$

Uma vez determinados os dois pontos $P_{1}$ e $P_{2}$ podemos determinar a trajetória definida por eles, lembrando que a origem do sistema de referência é o centro da Terra, como indicado nas figuras 3.5 e 4.2. As coordenadas usadas para descrever esta trajetória são cartesianas. Assim, a reta definida por $P_{1}\left(x_{1}, y_{1}, z_{1}\right)$ e $P_{2}\left(x_{2}, y_{2}, z_{2}\right)$ é dada por:

$$
(x, y, z)=\left(x_{1}, y_{1}, z_{1}\right)+\lambda\left(x_{1}-x_{2}, y_{1}-y_{2}, z_{1}-z_{2}\right)
$$

$\operatorname{com} \lambda \in \Re$.

Definindo $x_{1}-x_{2}=\Delta x, y_{1}-y_{2}=\Delta y$ e $z_{1}-z_{2}=\Delta z$, temos a profundidade do detector para cada trajetória dada pela distância entre os pontos $P_{1}$ e $P_{2}$ : 


$$
\Delta=\sqrt{(\Delta x)^{2}+(\Delta y)^{2}+(\Delta z)^{2}}
$$

Para determinar o ponto de entrada do neutrino na superfície da Terra, $P_{T}\left(x_{T}, y_{T}, z_{T}\right)$, queremos $\lambda$ tal que:

$$
x^{2}+y^{2}+z^{2}=R^{2}
$$

onde $R$ é o raio da Terra, lembrando que ela é aproximada por uma esfera. Sendo assim, ao substituirmos E.12 em E.10, temos:

$$
\left(x_{1}+\lambda \Delta x\right)^{2}+\left(y_{1}+\lambda \Delta y\right)^{2}+\left(z_{1}+\lambda \Delta z\right)^{2}=R^{2}
$$

Defindo também $R_{1}^{2}=x_{1}^{2}+y_{1}^{2}+z_{1}^{2}$ e $\vec{R}_{1} \cdot \vec{\Delta}=x_{1} \Delta x+y_{1} \Delta y+z_{1} \Delta z$, chegamos à seguinte equação em $\lambda$ :

$$
\Delta^{2} \lambda^{2}+2\left(\vec{R}_{1} \cdot \vec{\Delta}\right) \lambda-\left(R^{2}-R_{1}^{2}\right)=0,
$$

cuja solução é dada por:

$$
\lambda=-\frac{\vec{R}_{1} \cdot \vec{\Delta}}{\Delta^{2}} \pm\left[\frac{\left(\vec{R}_{1} \cdot \vec{\Delta}\right)^{2}}{\Delta^{4}}+\frac{\Delta^{2}\left(R^{2}-R_{1}^{2}\right)}{\Delta^{2}}\right]^{1 / 2}
$$

A orientação da trajetória parte do ponto de entrada na Terra, passando por $\mathrm{P}_{1}$ e chegando a $\mathrm{P}_{2}$. Por isto tomamos apenas uma das soluções:

$$
\lambda_{T}=-\frac{\vec{R}_{1} \cdot \vec{\Delta}}{\Delta^{2}}+\left[\frac{\left(\vec{R}_{1} \cdot \vec{\Delta}\right)^{2}}{\Delta^{4}}+\frac{\Delta^{2}\left(R^{2}-R_{1}^{2}\right)}{\Delta^{2}}\right]^{1 / 2}
$$

Obtemos $\mathrm{P}_{T}\left(x_{T}, y_{T}, z_{T}\right)$ substituindo $\lambda_{T}$ na equação E.10.

Dados dois pontos quaisquer pertencentes à reta determinada acima, $P_{a}\left(x_{a}, y_{a}, z_{a}\right)$ e $P_{B}\left(x_{b}, y_{b}, z_{b}\right)$, podemos determinar a relação entre a variação em $\lambda$ e distância entre eles $\left(\Delta_{a b}\right)$, dada por:

$$
\Delta_{a b}=\left|\lambda_{a}-\lambda_{b}\right| \Delta
$$


obtida a partir das equação E.10 e E.11. Desta forma, podemos determinar qualquer ponto entre o ponto de entrada na Terra e o detector, aplicando $\lambda_{a}$ na equação E.10, determinado a seguir:

$$
\lambda_{a}=\lambda_{T}-\frac{\Delta_{a T}}{\Delta}
$$

onde $\Delta_{a T}$ é a distância entre o ponto $P_{a}$ e o ponto $P_{T}$. 


\section{Referências Bibliográficas}

[1] K. Nakamura et al. (Particle Data Group), J. Phys. G 37, 075021 (2010);

[2] S. Weinberg, Physical Review D, 13, 974 (1976);

[3] S. Weinberg, Physical Review D, 19, 1277 (1979);

[4] S. P. Martin, in Perspectives on Supersymmetry, Ed. Kane, G.L. (1997); arXiv:hep-ph/9709356;

[5] G. F. Giudice, R. Rattazzi, Physics Reports, 322, 419-499 (1999);

[6] I. F. M. Albuquerque, G. Burdman, and Z. Chacko, Physical Review Letters, 92, 221802-1 (2004);

[7] I. F. M. Albuquerque, G. Burdman, Z. Chacko, Physical Review D, 75, 035006 (2007);

[8] J. Ahrens et. al. (The Ice Cube Collaboration), Nuclear Physics B, Proceedings Supplements, 118, 371 (2003);

[9] http://jemeuso.riken.jp/en/index.html;

[10] T. Ebisuzaki, for the JEM-EUSO Collaboration, AIP Conf. Proc. 1367:120-125 (2011); arxiv:1101.1909v1 [astro-ph];

[11] E. Waxman, J. N. Bahcall, Physical Review D, 59, 023002 (1998);

[12] J. N. Bahcall, E. Waxman, Physical Review D, 64, 023002 (2001);

[13] K. Mannheim, R. J. Protheroe, J. P. Rachen, Physical Review D, 63, 023003 (2001); 
[14] R. Gandhi, C. Quigg, M. H. Reno, I. Sarcevic, Astroparticle Physics, 5, 81-110 (1996);

[15] Yu Seon Jeong, Mary Hall Reno, Physical Review D, 82, 033010 (2010); arXiv:1007.1966v1 [hep-ph];

[16] http://cernlib.web.cern.ch/cernlib/;

[17] S. I. Dutta, M. H. Reno, I. Sarcevic, D. Seckel, Physical Review D, 63, 094020 (2001);

[18] I. F. M. Albuquerque, J. Lamoureux, G. F. Smoot, The Astrophysical Journal Supplement Series, 141, 195-209 (2002);

[19] S. Eidelman, et. al. (Particle data Group), Physics Letters B, 592, 1-5 (2004);

[20] Robert Martin Eisberg, Fundamentals of Modern Physics, New York London, John Wiley \& Sons, Inc, Wiley International Edition (1964);

[21] Raymond A. Serway, Clement J. Moses, Curt A. Moyer, Modern Physics, Second Edition, Saunders College Publishing, Harcourt Brace College Publishers (2005);

[22] D. E. Groom, N. V. Mokhov, S. I. Striganov, Atomic Data and Nuclear Data Tables, 78, 183-356 (2001);

[23] R. P. Kokoulin, A. A. Petrukhin, Acta. Phys. Acad. Sci. Hung., Suppl., 29, 277 (1970);

[24] H. Abramowicz, E. M. Levin, A. Levy, U. Maor, Physics Letters B, 269, 465-476 (1991);

[25] H. Abramowicz, A. Levy, hep-ph/9712415 (2004);

[26] F. Halzen, D. Hooper, JCAP 0401, 002 (2004);

[27] D. F. Cowen, Journal of Physics: Conference Series, 60, 227 (2007);

[28] E. Bugaev, T. Montaruli, Y. Shlepin and I. Sokalski, Astroparticle Physics, 21, 491 (2004); 
[29] Vernon Barger, Roger Phillips, Collider physics, Addison-Wesley (1987);

[30] S. I. Dutta, M. H. Reno, I. Sarcevic, Physical Review D, 62, 123001 (2000);

[31] P. R. Crotty, High-energy neutrino fluxes from supermassive dark matter, Tese de doutorado, Universidade de Chicago, Chicago, Illinois (2002);

[32] J. G. Learned, S. Pakvasa, Astroparticle Physics, 3, 276 (1995);

[33] F. Kakimoto et al., Nuclear Instruments and Methods in Physics Rese$\operatorname{arch~A,~372,~} 527-533$ (1996);

[34] M. Ave, M. Bohacova, K. Daumiler, P. Di Carlo, C. Di Giulio et al., AIP Conference Proceedings, 1367, 29 (2011);

[35] R. Abbasi et al., Physical Review D, 83, 012001 (2011);

[36] F. Halzen, Invited Review Article: IceCube: An Instrument for neutrino astronomy, S. R. Klein, Review of Scientific Instruments, 81, 081101 (2010);

[37] IceCube - Preliminary Design Document by Section: http://www.icecube.wisc.edu/science/publications/pdd/index.php;

[38] http://www.icecube.wisc.edu/index.php; 


\section{Lista de Figuras}

1.1 Esquema da interação de neutrinos cosmológicos com a Terra produzindo duas NLSPs que atingem um telescópio de neutrinos. . . . 5

1.2 Esquema da interação de neutrinos cosmológicos com a Terra, produzindo duas NLSPs que decaem dentro do planeta. . . . . . . . 7

1.3 Esquema da interação de neutrinos cosmológicos com a Terra e produzindo duas NLSPs que decaem na atmosfera após atravessarem o planeta. . . . . . . . . . . . . . . 8

2.1 A área em preto representa o limite de Waxman e Bahcall (WB) desde o limite considerando evolução cosmológica (limite inferior) até o limite não considerando evolução cosmológica (limite superior). O limite de Mannheim, Protheroe e Rachen (MPR) para fontes opticamente "finas" é representado pela linha vermelha e no caso de fontes "espessas", pela linha azul. . . . . . . . . . . . 12

2.2 Seção de choque inclusiva de corrente carrega da interação neutrinonúcleon para a produção de léptons. . . . . . . . . . . . . . . 15

2.3 Diagramas de Feynman para a produção de partículas supersimétricas por meio de interações neutrino-núcleon. (a) Interação mão esquerda mão esquerda; (b) interação mão esquerda mão direita; (c) e (d) correspondem à corrente neutra. Há diagramas análogos para anti-neutrinos assim como para quarks iniciais do tipo strange e charm. . . . . . . . . . . . . . . . . . . . 16 
2.4 Seção de choque da interação neutrino-núcleon para a produção de partículas supersimétricas. São considerados três cenários para massa do squark produzido na interação neutrino-núcleon: 300 $\mathrm{GeV}, 600 \mathrm{GeV}$ e $900 \mathrm{GeV}$. Estas curvas são compatíveis com [6]. Também é apresentada a curva da a seção de choque para produção de léptons em preto. . . . . . . . . . . . . . . . . . . . 18

2.5 Probabilidade de sobrevivência do neutrino ao atravessar a Terra em função do cosseno do ângulo de incidência, para 6 valores de energia. Foi tomado um ponto de referência fixo a cerca de $2 \mathrm{~km}$ de profundidade. . . . . . . . . . . . . . . . . 20

2.6 Probabilidade do neutrino interagir, produzindo um slépton, ao atravessar a Terra em função do cosseno do ângulo de incidência, para 6 valores de energia de incidência do neutrino. Foi tomado como alvo um ponto fixo na Terra, a cerca de $2 \mathrm{~km}$ de profundidade. 21

2.7 Probabilidade resultante da convolução das probabilidades de sobrevivência do neutrino (levando em conta a probabilidade de produzir múons), até o ponto em que o stau produzido pode chegar ao detector, e de interação do mesmo (produção de stau), em função do cosseno do ângulo de incidência, para 6 valores de energia de incidência do neutrino. Foi utilizado um ponto de referência fixo na Terra, a cerca de $2 \mathrm{~km}$ de profundidade. . . . . . . . . . . 22

2.8 Esquema da Terra com os parâmetros utilizados para a determinação do seu perfil de densidade. A distância $a$ representa a profundidade do detector. . . . . . . . . . . . . . . . 23

2.9 Perfil de densidade da Terra encontrado por uma partícula que incide sobre o planeta em diferentes ângulos. A distância percorrida é medida a partir da superfície da Terra até o detector, a aproximadamente $2 \mathrm{~km}$ de profundidade. . . . . . . . . . . . . 24

2.10 Perda de energia média (por radiação) de múons em função de sua energia. São apresentadas as contribuições relevantes: bremsstrahlung, produção de pares e interação fotonuclear. . . . . . . . . 26

2.11 Perda de energia (por radiação) do tau em função de sua energia. São apresentadas as contribuições relevantes: bremsstrahlung, produção de pares e interação fotonuclear. . . . . . . . . . . . . . 26 
2.12 Perda de energia média (por radiação) do slépton em função de sua energia. São apresentadas as contribuições relevantes: bremsstrahlung, produção de pares e interação fotonuclear. . . . . . . . 27

2.13 Perda total de energia por radiação do múon, tau e slépton em função de suas energias. São apresentadas às somas das três contribuições relevantes: bremsstrahlung, produção de pares e interação fotonuclear. . . . . . . . . . . . . . . . . . . . . . 28

2.14 Alcance máximo para múons de diversas energias. Foi considerado que as partículas incidem sobre a superfície da Terra. . . . . . . 36

2.15 Alcance máximo para taus de diversas energias. Foi considerado que as partículas incidem sobre a superfície da Terra. . . . . . . . 36

2.16 Alcance máximo para sléptons de diversas energias. Foi considerado que as partículas incidem sobre a superfície da Terra. . . . . 37

2.17 Em vermelho é apresentada a distribuição do ponto de produção dos staus no interior da Terra para um neutrino incidente com energia de $1 \times 10^{9} \mathrm{GeV}$ e ângulo de 180 graus. O eixo $x$ representa o ponto de produção em relação ao ponto de incidência na superfície da Terra. A curva em preto corresponde à probabilidade de o neutrino interagir relativa à produção de NLSPs (equação 2.18). 39

2.18 Eventos por $\mathrm{km}^{2}$ por ano num detector localizado a cerca de 2 $\mathrm{km}$ de profundidade. São apresentadas as curvas para três massas do squark produzido na interação neutrino-núcleon: 300, 600 e $900 \mathrm{GeV}$. É apresentado também, em preto, o limite de WaxmanBahcall, que é adotado como o fluxo de neutrinos. . . . . . . . . . 40

2.19 Energia de incidência das NLSPs no detector para três valores de massa do squark produzido na interação neutrino-núcleon: 300, 600 e $900 \mathrm{GeV}$

3.1 Probabilidade de sobrevivência do stau em função da distância percorrida para quatro valores de $\sqrt{F}$ e energia do neutrino de $5 \times 10^{6} \mathrm{GeV} \ldots \ldots \ldots \ldots \ldots$

3.2 Probabilidade de sobrevivência do stau em função da distância percorrida para quatro valores de energia do neutrino $\left(E_{\nu}\right)$ e $\sqrt{F}=$ $1 \times 10^{6} \mathrm{GeV} \ldots \ldots \ldots \ldots \ldots$ 
3.3 Distância média percorrida pelo tau, antes de decair, em função de sua energia. . . . . . . . . . . . . . . . . . . . . . . . . 48

3.4 Distribuição $\frac{d n}{d z}$ em função de $z$, que relaciona as energias dos produtos de decaimento do tau com a energia do mesmo. . . . . . . . 50

3.5 Esquema da geometria utilizada para geração das trajetórias que passam por um telescópio de neutrinos a cerca de $2 \mathrm{~km}$ de profundidade, cuja forma foi aproximada por um cilindro. . . . . . . . . 52

3.6 Fluxos por ano no volume de um telescópio de neutrinos de $1 \mathrm{~km}^{3}$. Também é apresentado o fluxo do total de NLSPs produzidas e o limite de Waxman e Bahcall. . . . . . . . . . . . . . . . . . . 57

3.7 Distribuições de energia no detector para taus e múons, tomandose três valores de massa squark produzido na interação neutrinonúcleon: 300,600 e $900 \mathrm{GeV} . \sqrt{F}=1 \times 10^{5} \mathrm{GeV}$. . . . . . . 59

3.8 Número de regenerações médio do tau, relativas aos taus que atingem o detector, em função da energia do neutrino incidente, para $\sqrt{F}=1 \times 10^{5} \mathrm{GeV}$ e três valores de $m_{\tilde{q}}: 300,600$ e $900 \mathrm{GeV}$. . . 60

3.9 Fluxos de partículas por ano no volume de um telescópio de neutrinos de $1 \mathrm{~km}^{3}$, como o IceCube, em função de $\sqrt{F}$. . . . . . . . 61

3.10 Energia média de taus, múons e staus (de cima para baixo) chegando no volume de um telescópio de neutrinos de $1 \mathrm{~km}^{3}$. As barras de incerteza correspondem aos desvios-padrão das distribuições. . . 62

3.11 Fluxo de taus para telescópios de neutrinos de raios $2 \mathrm{~km}$ (acima) e $4 \mathrm{~km}$ (abaixo) maiores do que o IceCube. A massa do squark produzido na interação neutrino-núcleon é $300 \mathrm{GeV}$. . . . . . . . 66

3.12 Fluxo de taus para telescópios de neutrinos de raios $2 \mathrm{~km}$ (acima) e $4 \mathrm{~km}$ (abaixo) maiores do que o IceCube. A massa do squark produzido na interação neutrino-núcleon é $600 \mathrm{GeV}$. . . . . . . . 67

3.13 Fluxo de taus para telescópios de neutrinos de raios $2 \mathrm{~km}$ (acima) e $4 \mathrm{~km}$ (abaixo) maiores do que o IceCube. A massa do squark produzido na interação neutrino-núcleon é $900 \mathrm{GeV}$. . . . . . . . 68

3.14 Distribuições de energia no detector para taus e múons, com $\sqrt{F}=$ $1 \times 10^{5} \mathrm{GeV} \ldots \ldots \ldots \ldots \ldots$

3.15 Distribuições de energia no detector para taus e múons, para três valores de massa squark produzido na interação neutrino-núcleon: 300,600 e $900 \mathrm{GeV} . \sqrt{F}=1 \times 10^{5} \mathrm{GeV} \ldots \ldots \ldots$ 
3.16 Regenerações do tau em função da energia do neutrino incidente para 3 volumes de telescópio de neutrinos, para a massa do squark de $300 \mathrm{GeV}$. . . . . . . . . . . . . . . . . . . . . . 71

3.17 Fluxo de staus e de taus mais múons em 3 telescópios de neutrinos de diferentes volumes. . . . . . . . . . . . . . . . . . . 72

3.18 Fluxos de partículas por ano em telescópios de neutrinos similares ao IceCube, porém com raios $2 \mathrm{~km}$ (acima) e $4 \mathrm{~km}$ (abaixo) maiores, para $m_{\tilde{q}}=300 \mathrm{GeV}$. . . . . . . . . . . . 73

3.19 Fluxos de partículas por ano em telescópios de neutrinos similares ao IceCube, porém com raios $2 \mathrm{~km}$ (acima) e $4 \mathrm{~km}$ (abaixo) maiores, para $m_{\tilde{q}}=600 \mathrm{GeV}$. . . . . . . . . . . . . . . . . . 74

3.20 Fluxos de partículas por ano em telescópios de neutrinos similares ao IceCube, porém com raios $2 \mathrm{~km}$ (acima) e $4 \mathrm{~km}$ (abaixo) maiores, para $m_{\tilde{q}}=900 \mathrm{GeV}$.

3.21 Fração de eventos de taus em função da energia do neutrino incidente. São apresentados também a soma de todos os taus que atingem o detector e aqueles que passam por ele sem decair. Os outros casos estão descritos no texto.

3.22 Distribuição de energia de taus no IceCube, considerando diferentes assinaturas desta partícula no detector. As normalizações são dadas pelas frações de eventos independentes de fluxo. . . . . . . . 81

3.23 Distribuição de energia de taus no IceCube, considerando diferentes assinaturas desta partícula no detector. As normalizações são dadas pelas frações de eventos independentes de fluxo. . . . . . . . 82

3.24 Média de regenerações para taus quando não se leva em conta a produção de NLSPs. São considerados 3 possíveis volumes. . . . . 83

4.1 Desenho esquemático do experimento JEM-EUSO. Figura obtida de $[9] \ldots \ldots \ldots \ldots 6$

4.2 Esquema da geometria utilizada para geração das trajetórias das partículas no caso do telescópio JEM-EUSO. $\mathrm{P}_{1}$ está na superfície (S) da Terra e $\mathrm{P}_{2}$ pode estar na superfície lateral ou na superfície superior do tronco de cone definida pelo limite superior da atmosfera. $\mathrm{P}_{3}$ indica o ponto de intersecção da trajetória com a superfície da Terra. . . . . . . . . . . . . . . . . 87 
4.3 Eventos por ano em função da energia do neutrino incidente, tendo como referência o campo de visão do telescópio JEM-EUSO. Em vermelho temos todos os staus; em laranja e verde, os decaimentos de staus e taus que ocorrem dentro do campo de visão do detector; círculos em ciano e triângulos pretos, decaimentos de staus e taus que ocorrem após o campo de visão do detector; em azul e roxo, decaimentos de NLSPs e de taus em coincidência dentro do campo de visão do detector. Também é apresentado o limite de Waxman e Bahcall. . . . . . . . . . . . . . . . . . . . . . . . 92

4.4 Distribuição de energia dos staus que decaem em coincidência na atmosfera. . . . . . . . . . . . . . . 93

4.5 Coincidências de decaimentos de staus por ano, dentro do campo de visão do JEM-EUSO, em função de $\sqrt{F}$. . . . . . . . . . . . . 94

4.6 Energia média dos staus que decaem em coincidências dentro do campo de visão do JEM-EUSO, em função de $\sqrt{F}$. As incertezas dos pontos representam os desvios-padrão das distribuições. . . . . 94

4.7 Coincidências de decaimentos de staus por ano, dentro do campo de visão do JEM-EUSO, em função de $\sqrt{F}$, quando $E_{\tilde{\tau}} \geq 10^{8}, 10^{9}, 10^{10}$ $\mathrm{GeV}$.

4.8 Distribuições diferença de tempo entre os instantes de decaimentos dos pares de staus dentro do campo de visão do JEM-EUSO, para diferentes valores de $\sqrt{F}$ e restrições de energia mínima destas partículas. . . . . . . . . . . . . . . . . . . . 98

4.9 Distribuições da distância entre os pontos de decaimento dos pares de staus quando estes decaimentos ocorrem dentro do campo de visão do detector, para diferentes valores de $\sqrt{F}$ e restrições de energia para estas partículas. . . . . . . . . . . . . . . . 99

5.1 Comparação entre os fluxos de staus e a soma dos fluxos de taus e múons, por ano num telescópio de neutrinos de $1 \mathrm{~km}^{3}$, como o IceCube. Para os taus e múons (provenientes do decaimento do tau), $\sqrt{F}=1 \times 10^{5} \mathrm{GeV}$. No caso dos staus, $\sqrt{F}=5 \times 10^{7} \mathrm{GeV}$. É apresentado também um fluxo extrapolado de neutrinos atmosféricos. 104

A.1 Esquema do telescópio de neutrinos IceCube, em escala, obtido de [38]. . . . . . . . . . . . . . . . . . . . . . . . . 112 
A.2 À esquerda é ilustrado um sinal provocado por múons e à direita, pela interação do neutrino do elétron ou do tau. Figura btida de [36] . . . . . . . . . . . . . . . . . . . . . . 112

A.3 Eventos simulados: (a) $\nu_{\mu} N \rightarrow \mu X$; (b) $\nu_{e} N \rightarrow$ cascata; (c) double bang, $\nu_{\tau} N \rightarrow$ cascata $_{1}+\nu_{t} a u N \rightarrow$ cascata $_{2}$. Figura obtida de [36]. 113

B.1 Seção de choque inclusiva de corrente carrega da interação neutrinonucleon para a produção de múons. Em preto são apresentados os pontos obtidos com o cálculo da seção de choque e em vermelho, os pontos obtidos com a curva ajustada. . . . . . . . . . . . . 116

B.2 Parametrização da seção de choque da interação neutrino-nucleon para a produção de partículas supersimétricas. Neste caso a massa do squark é $300 \mathrm{GeV}$. . . . . . . . . . . . . . . . . . . . . 118

B.3 Parametrização da perda de energia (por radiação) de múons ao atravessar a Terra em função da energia de incidência. É apresentada a soma das contribuições relevantes: por bremsstrahlung, por produção de pares e por interação fotonuclear. . . . . . . . . . . . 120

B.4 Parametrização da perda de energia (por radiação) de taus ao atravessar a Terra em função da energia de incidência. É apresentada a soma das contribuições relevantes: por bremsstrahlung, por produção de pares e por interação fotonuclear. . . . . . . . . . . . 121

B.5 Parametrização da perda de energia (por radiação) do slépton de $150 \mathrm{GeV}$ ao atravessar a Terra em função da energia de incidência. É apresentada a soma das contribuições relevantes: por bremsstrahlung, por produção de pares e por interação fotonuclear. . . . . 122

B.6 Parametrização de у. . . . . . . . . . . . . . . . . . . . . . . . . 127

C.1 Comparação entre o histograma (em vermelho) obtido com o algoritmo criado para gerar números aleatórios que seguem uma distribuição qualquer e a distribuição de probabilidade dada por $p=p_{s_{\nu}}\left(1-p_{s_{\nu}}\right)$, em função da distância entre a superfície (no ponto de incidência) e o detector. A curva em preto é a distribuição calculada analiticamente. . . . . . . . . . . . . . . . 130

C.2 Distribuição $\frac{d n}{d z}$ em função de $z$. Em preto tem-se a função dada pela equação (3.11) e em vermelho, a respectiva distribuição aleatória.131 
D.1 Esquema da colisão entre as partículas 1 e 2, produzindo após a colisão, as partículas 3 e 4. A situação é ilustrada nos referenciais do laboratório (Lab) e do centro de momentos (CM) . . . . . . . 137 


\section{Lista de Tabelas}

1.1 Supermultipletos chirais no modelo padrão supersimétrico mínimo. Os campos de spin zero são escalares complexos e os campos de spin $1 / 2$ são férmions de Weyl de mão esquerda de duas componentes.

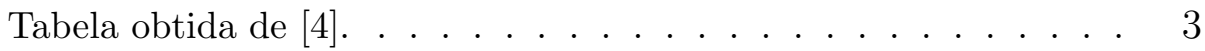

1.2 Supermultipletos de gauge no modelo padrão supersimétrico mínimo. Tabela obtida de $[4] . \ldots \ldots \ldots$

2.1 Valores dos parâmetros ALLM 97 retirados da referência [25]. . . . 34

2.2 Eventos de NLSPs por $\mathrm{km}^{2}$ por ano no telescópio de neutrinos posicionado a cerca de $2 \mathrm{~km}$ de profundidade na Terra. . . . . . . 40

3.1 Funções $g_{0}$ e $g_{1}$ para a distribuição de energia do neutrino do tau proveniente do decaimento do tau, em função de $z=E_{\nu_{\tau}} / E_{\tau}$. X indica todos os hádrons excetuando-se os píons $(\pi), \rho$ e $a_{1} \cdot r_{i}=$ $m_{i}^{2} / m_{\tau}^{2}$. A função $\theta$ é a função degrau. Dados obtidos de [30]. . . . 50

3.2 Taxas de taus, múons e staus por ano em um telescópio de neutrinos de $1 \mathrm{~km}^{3}$ situado a cerca de $2 \mathrm{~km}$ de profundidade, para três valores de massa do squark produzido na interação neutrinonúcleon. No caso dos staus a taxa apresentada é o número de pares. $\sqrt{F}=1 \times 10^{5} \mathrm{GeV}$ para taus e múons e $5 \times 10^{7} \mathrm{GeV}$ para staus. . . . . . . . . . . . . . . . . 5 58

3.3 Taxas de eventos por ano para os volumes e massas do squark especificados. Para staus, $\sqrt{F}=5 \times 10^{7} \mathrm{GeV}$; para taus e múons, $1 \times 10^{5} \mathrm{GeV} \ldots \ldots \ldots \ldots$. . . . . . . . . . . 69 
3.4 Número de taus por ano em telescópios de neutrinos de 3 volumes, normalizados com 3 diferentes limites para o fluxo de neutrinos: $\mathrm{WB}, \mathrm{MPR}_{f}$ (fontes finas) e $\mathrm{MPR}_{e}$ (fontes espessas). A taxa de eventos é apresentada independentemente do fluxo de neutrinos, ou seja, a fração $f_{N}$ dos eventos gerados que chegam ao detector, bem como para as diferentes assinaturas do tau: Lollipop, InvLollipop, Tautsipop e Double Bang. Além disso, são apresentadas as taxas para o total de taus e para os taus que atravessam o volume do detector. . . . . . . . . . . . . . . . 79

3.5 Idem à tabela 3.4, mas agora impondo um limiar de energia de $2 \times 10^{6} \mathrm{GeV} \ldots \ldots \ldots \ldots \ldots . \ldots \ldots$

4.1 Fluxo máximo de NLSPs por ano na atmosfera para diferentes situações de decaimento, com $\sqrt{F}=3 \times 10^{6} \mathrm{GeV}$ para $m_{\tilde{q}}$ de $300 \mathrm{e}$ $600 \mathrm{GeV}$ e com $\sqrt{F}=2 \times 10^{6}$ para $m_{\tilde{q}}=900 \mathrm{GeV}$. "Total" corresponde a todos os staus produzidos; "Não decaem", aos decaimentos que ocorrem fora do campo de visão do detector; "Decaem (total)", ao total de decaimentos dentro do campo de visão do detector; e "Par decai", aos decaimentos em coincidência dos staus do par dentro do campo de visão do detector com geração de um par de taus. . . . . . . . . . . . . . . . . . . 93

4.2 Eventos por ano quando são impostas as restrições de energia. Também são apresentados as taxas de decaimentos dos taus. Para $\sqrt{F}=7 \times 10^{5} \mathrm{GeV}, E_{\tilde{\tau}} \geq 10^{8} \mathrm{GeV}$; para $\sqrt{F}=3 \times 10^{5} \mathrm{GeV}$, $E_{\tilde{\tau}} \geq 10^{9} \mathrm{GeV}$; e para $\sqrt{F}=2 \times 10^{5} \mathrm{GeV}, E_{\tilde{\tau}} \geq 10^{10} \mathrm{GeV}$. . . . 97

5.1 Taxas de taus e múons, resultantes do decaimento de NLSPs, por ano em um telescópio de neutrinos de $1 \mathrm{~km}^{3}$ situado a cerca de $2 \mathrm{~km}$ de profundidade, com $\sqrt{F}=1 \times 10^{5} \mathrm{GeV}$. Foram adotados dois valores de energia mínima do neutrino incidente: $10^{7}$ e $10^{8} \mathrm{GeV} .105$

5.2 Eventos de taus e múons por ano nos volumes especificados, para $E_{\nu}>10^{8} \mathrm{GeV}$, massas do squark de 300,600 e $900 \mathrm{GeV}$ e $\sqrt{F}=$ $1 \times 10^{5} \mathrm{GeV} \ldots \ldots \ldots \ldots \ldots$ 
B.1 Valores dos parâmetros para o ajuste da seção de choque inclusiva de corrente carregada para a interação neutrino-nucleon na produção de léptons. . . . . . . . . . . . . . . . . . . . 117

B.2 Valores dos parâmetros para o ajuste da seção de choque de produção de partículas supersimétricas para $m \tilde{q}=300 \mathrm{GeV}$. Há parâmetros que não apresentam incerteza. Nestes casos ela está a pelo menos dois algarismos significativos do último apresentado. . . . . . . . . 119

B.3 Valores dos parâmetros para o ajuste da perda de energia para múons. . . . . . . . . . . . . . . . . . . . 123

B.4 Valores dos parâmetros para o ajuste da perda de energia para taus.124

B.5 Valores dos parâmetros para o ajuste da perda de energia para sléptons. . . . . . . . . . . . . . . . . . . . . 125

B.6 Valores de $y$ em função de $E_{\nu}$. Dados de [14]. . . . . . . . . . . 126 